\title{
DEVELOPMENT OF IN-VEHICLE COLLISION WARNING SYSTEM FOR INTERSECTIONS
}

\author{
by \\ Essam Mohamed S.A.E.A. Dabbour, M. A. Sc., P. Eng. \\ Master of Applied Science, Ryerson University, Toronto, Ontario (2003) \\ Bachelor of Science, Alexandria University, Alexandria, Egypt (1988)
}

A dissertation presented to Ryerson University

\author{
in partial fulfillment of the \\ requirements for the degree of \\ Doctor of Philosophy (Ph. D.) \\ in the program of \\ Civil Engineering
}

(C) Essam Dabbour 2009 


\section{DECLARATION}

I hereby declare that I am the sole author of this dissertation. I authorize Ryerson University to lend this dissertation to other institutions or individuals for the purpose of scholarly research.

Signature

I further authorize Ryerson University to reproduce this dissertation by photocopying or by other means, in total or in part, at the request of other institutions or individuals for the purpose of scholarly research.

Signature 


\title{
DEVELOPMENT OF IN-VEHICLE COLLISION WARNING SYSTEM FOR INTERSECTIONS
}

A Doctor of Philosophy (Ph. D.) dissertation -2009

by

Essam Mohamed S.A.E.A. Dabbour, M. A. Sc., P. Eng.

This dissertation is presented to Ryerson University in partial fulfillment of the requirements for the degree of

Doctor of Philosophy (Ph. D.)

in the program of

Civil Engineering

\begin{abstract}
Most of the current collision warning systems are mainly designed to detect imminent rear-end, lane-changing or lane departure collisions. None of them was designed to detect imminent intersection collisions, which were found to cause more fatalities and injuries than other collisions. One of the most important factors that lead to intersection collisions is driver's human error and misjudgement. A main source for human errors is the insensitivity of human vision system to detect the speed and acceleration of approaching vehicles; and therefore, any algorithm for an intersection
\end{abstract}


collision warning system should give consideration to the speed and acceleration of all approaching vehicles to mitigate the insufficiency in the human vision system. Moreover, when designing any collision warning system, false warnings should be minimized to avoid nuisance for drivers that might lead to the loss of the system's reliability by potential users.

This research proposes a technology-independent intersection collision warning system that utilizes commercially-available detection sensors to detect approaching vehicles and measure their speeds and acceleration rates in order to estimate the time-to-collision and compare it to the time required for the turning vehicle to clear the paths of the approaching vehicles. To estimate the time required to clear the paths of the approaching vehicles, statistical models were developed to estimate the perception-reaction time for the driver of the turning vehicle as well as the rate of acceleration selected when departing the intersection. The statistical models include regression models that were calibrated from data collected through driving simulation as well as more-sophisticated artificial neural network models that are based on actual data collected from a specific driver on a specific vehicle. By comparing the time-to-collision with the time required for safe departure, the system triggers a warning message if an imminent collision is detected. The proposed system was validated by computer simulation to measure its reliability under different conditions and also to measure the impact of different components on the functionality and reliability of the system. Final conclusions are provided along with recommendations for further research. 


\section{ACKNOWLEDGEMENT}

This dissertation would not have been possible without the greatly-appreciated efforts of my supervisor, Dr. Said Easa, who gave me the inspiration, encouragement and support throughout my graduate studies program, either in the Master or Doctoral levels. Dr. Easa inspired me with the idea of this research and he kept improving it through very long hours that he dedicated from his valuable time for discussions, guidance and follow up. Dr. Easa also generously provided me with the financial support to complete my studies at times when I was in real need for that support. I would also like to thank all the members of my oral examination committee for their valuable comments that led to remarkable improvements in this dissertation. This includes Dr. Liping Fu from the University of Waterloo, Dr. Kamran Raahemifar from the Department of Electrical and Computer Engineering at Ryerson University, Dr. Anwar Hussain and Dr. Ali Mekky from the Department of Civil Engineering at Ryerson University.

This research was financially supported by a combination of different financial resources, including a scholarship and a discovery grant from the Natural Sciences and Engineering Research council of Canada, a scholarship from Transportation Association of Canada and an excellence scholarship from Ryerson University. At last, but not the least, I would like to thank all my family members, including my late father who passed away a few months ago, my mother, my wife and my both daughters. They all provided me with endless love, patience and encouragement throughout my study program. 


\section{DEDICATION}

This dissertation is dedicated to the three angels who shared with me the sacrifices required to complete it and were always there when I needed them to give me endless love, happiness and hope for better future. To my wife Enas and my two daughters Olaa and Abir. 


\section{TABLE OF CONTENTS}

CHAPTER 1: PROBLEM DEFENITION AND STUDY FRAMEWORK .................... 1

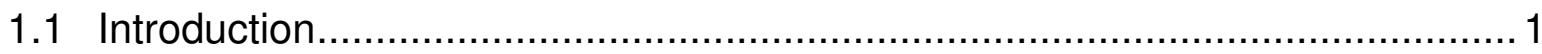

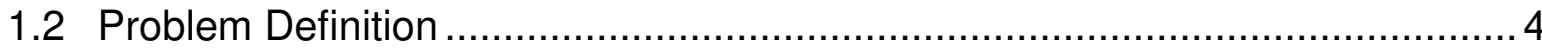

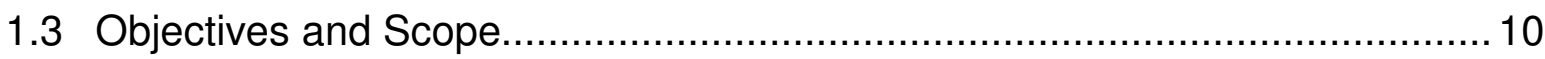

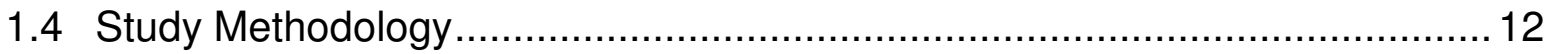

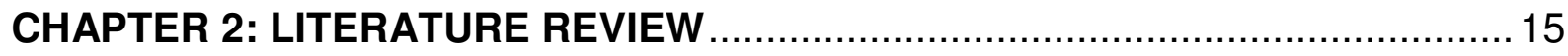

2.1 Human Factors at Intersections............................................................ 15

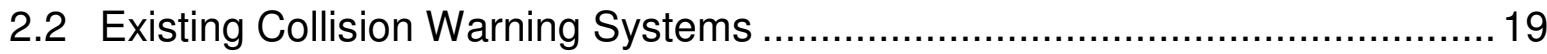

2.3 Intersection Collision Warning/Avoidance Systems ....................................2 21

2.4 Vehicle Acceleration Models .................................................................... 32

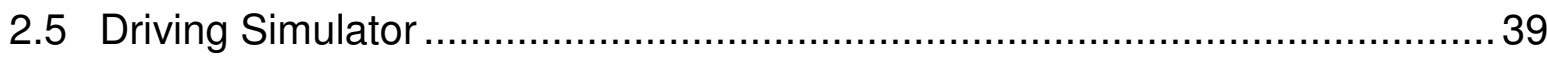

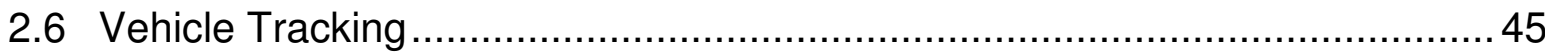

CHAPTER 3: ALGORITHM DESIGN FOR LEFT-TURNING VEHICLES …............47

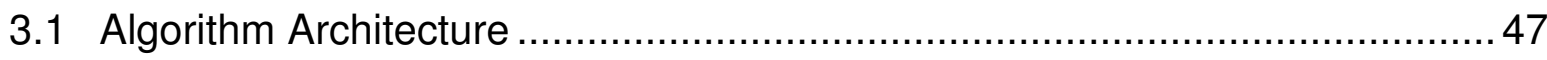

3.2 Specifications of Detection Sensor ......................................................... 49

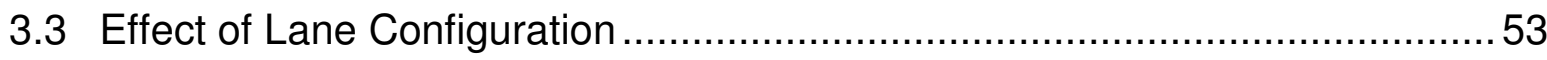

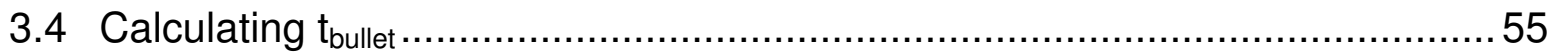

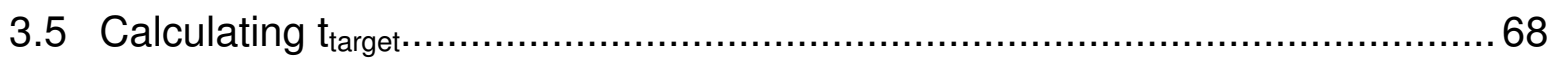

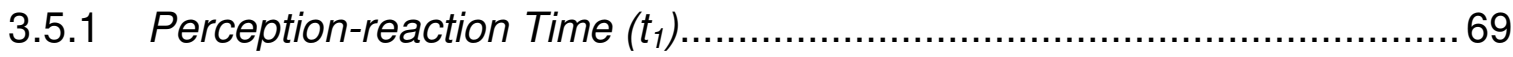

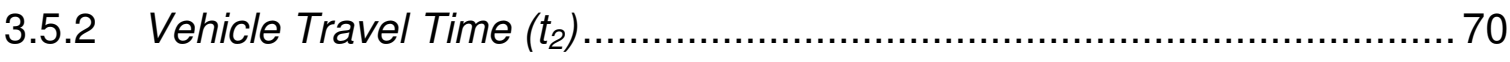

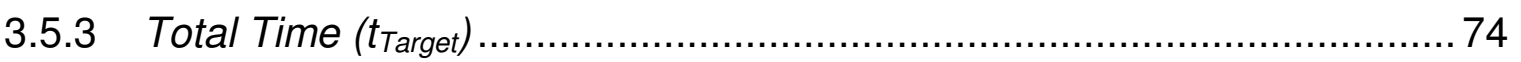

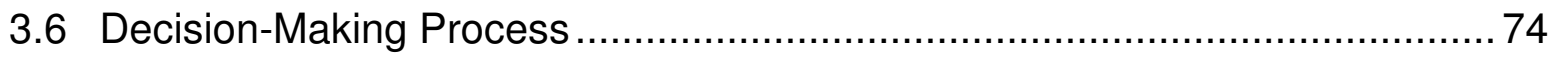




\section{CHAPTER 4: ALGORITHM DESIGN FOR SEMI-CONTROLLED INTERSECTIONS}

76

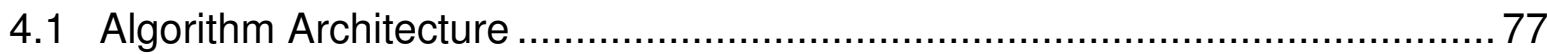

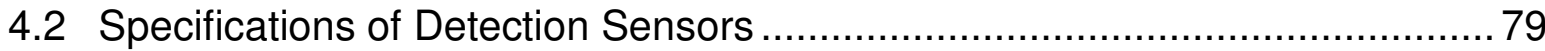

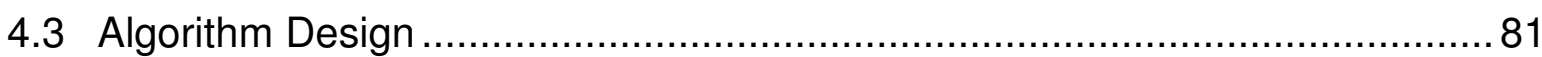

4.3.1 Determining Bullet Vehicle Location................................................. 83

4.3.2 Conflicting Vehicles have Perpendicular Paths ...................................92

4.3.3 Conflicting Vehicles Traveling on the Same Lane ................................96

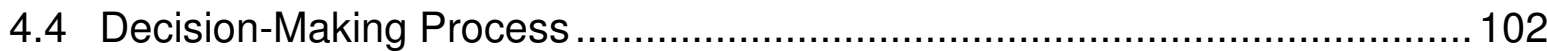

CHAPTER 5: DEVELOPMENT AND VALIDATION OF STATISTICAL MODELS 104

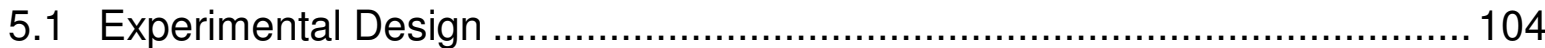

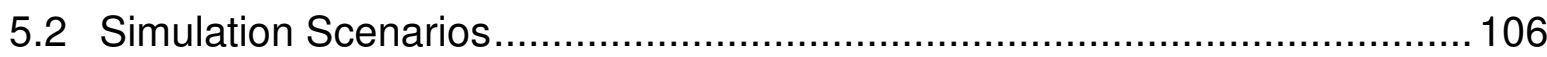

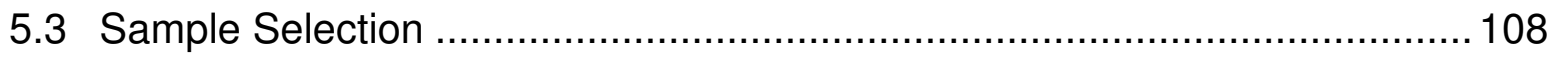

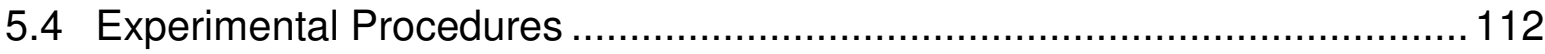

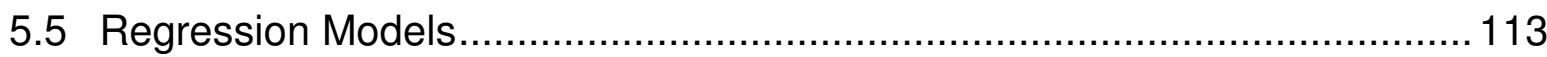

5.5.1 Left Turn Situation .................................................................. 114

5.5.2 Semi-Controlled Intersections ................................................... 117

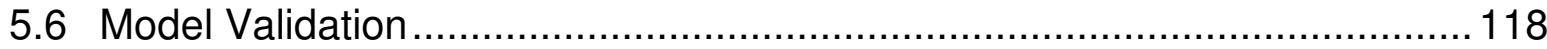

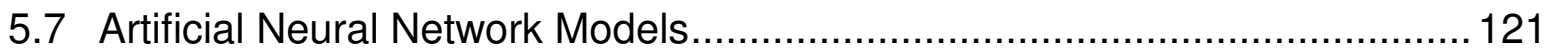

CHAPTER 6: SYSTEM VALIDATION AND SENSITIVITY ANALYSIS ...............130

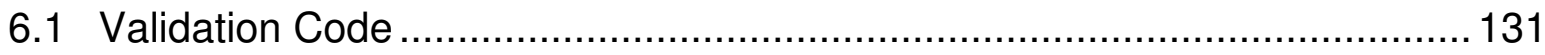

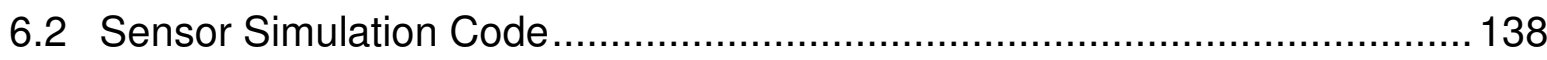

6.3 Effect of Measurement Precision.......................................................... 143 


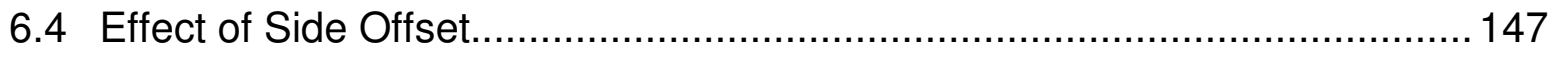

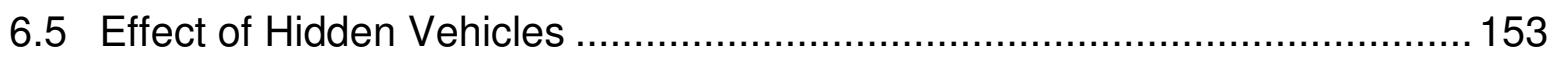

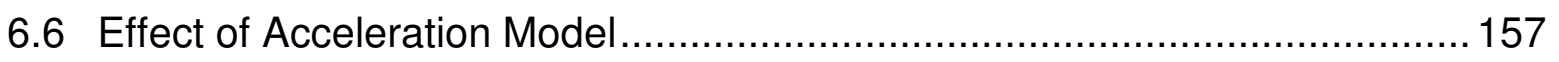

6.6.1 Bullet Vehicle Acceleration Model ................................................. 158

6.6.2 Target Vehicle Acceleration Model.................................................... 162

CHAPTER 7: SUMMARY, CONCLUSIONS AND RECOMMENDATIONS............167

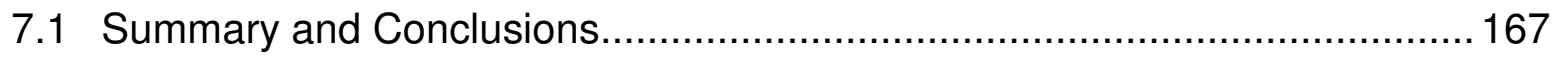

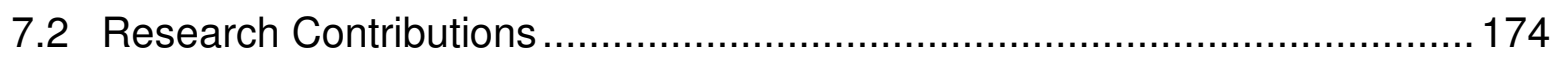

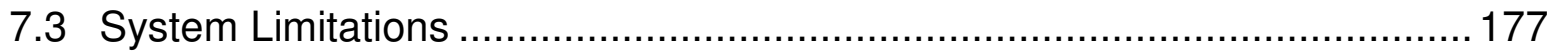

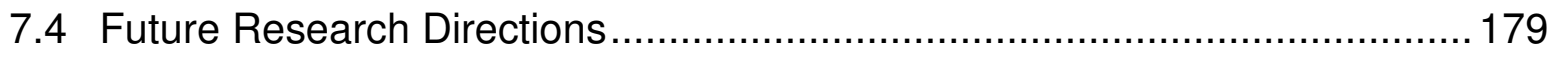

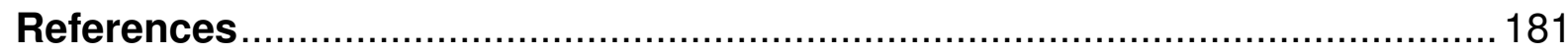

Glossary of Terms

Appendix “A"

Appendix “B”,

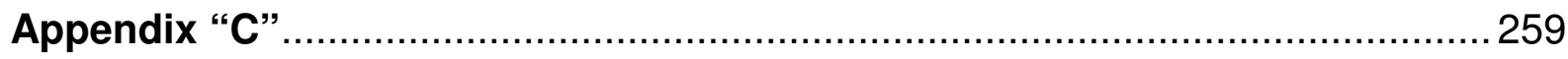

Appendix “D”'

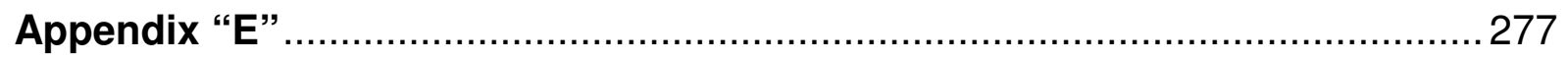

Appendix “F"

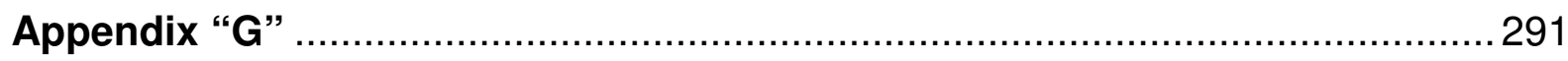




\section{LIST OF TABLES}

Table 1.1 Different collision types in Ontario

Table 2.1 Comparison for different intersection collision warning systems

Table 5.1 Licensed drivers in Ontario and Canada by age group (2003)

Table 5.2 Sample size for the experiment

Table 5.3 Summary of statistics for estimating $t_{1}$ (left turn)

Table 5.4 Summary of statistics estimating $c_{d}$ (left turn)

Table 5.5 Summary of statistics for estimating $t_{1}$ (departing a controlled minor road)

Table 5.6 Summary of statistics for estimating $c_{d}$ (departing a controlled minor road)

Table 5.7 Margin of error between calibration and validation samples

Table 5.8 Summary of validation statistics for all regression models

Table 5.9 Root-mean-squared errors for all sample drivers (ANN models)

Table 6.1 Effect of measuring precision on the side offset error

Table 6.2 Side offset for a vehicle on far lane (reflective point at near edge)

Table 6.3 Side offset for a vehicle on far lane (reflective point at centre)

Table 6.4 Side offset for a vehicle on far lane (reflective point at far edge)

Table 6.5 Effect of acceleration model on target time computation 


\section{LIST OF FIGURES}

Figure 1.1 Left-turn collisions at unsignalised and signalised intersections

Figure 1.2 Angle collisions at unsignalised and signalised intersections

Figure 1.3 Flow chart for research methodology

Figure 2.1 Time criterion for left-turn collisions

Figure 2.2 Maximum acceleration for different vehicles [Long 2000]

Figure 2.3 The experimental car used for simulation at Ryerson University

Figure 2.4 Screen shot from driving simulator by STISIM

Figure 3.1 Configuration of the proposed detection sensors

Figure 3.2 Flow chart for the left-turn algorithm

Figure 3.3 A typical roadway with less than 3 through lanes

Figure 3.4 A typical roadway with dedicated right-turn lane

Figure 3.5 A typical roadway with dedicated left-turn lanes

Figure 3.6 Schematic illustration of bullet vehicle locations at different time instances

Figure 3.7 Calculating distance and angle at time $T$

Figure 3.8 Calculating distance and angle at time $T+t$

Figure 3.9 The case where the radar beam detects a vehicle in the same direction

Figure 3.10 Calculating the distance traversed by the opposing vehicle

Figure 3.11 Calculating the distance at time $T+2 t$

Figure 3.12 Calculating the distance at time $T+3 t$

Figure 3.13 Observed acceleration profile [Wang et al. 2004] 
Figure 3.14 Calculating the distance $d_{f}$ and the offset $w_{f}$

Figure 4.1 Configuration of the proposed detection sensors

Figure 4.2 Conflict cases for a typical TWSC intersection

Figure 4.3 Schematic illustration of bullet vehicle locations at different time instances

Figure 4.4 Measuring the range and azimuth angle at time $T$

Figure 4.5 Measuring the range and azimuth angle at time $T+t$

Figure 4.6 Calculating bullet vehicle displacement during first time interval

Figure 4.7 Calculating side offset and distance between bullet and target vehicles

Figure 4.8 Conflict when bullet and target vehicles are traveling on the same lane

Figure 5.1 Driver populations in Ontario and Canada (2003) compared to sample

Figure 5.2 Typical multilayered feed-forward (2:2:1) ANN model

Figure 5.3 Feed-forward network with one-step ahead prediction

Figure 5.4 ANN prediction for the perception-reaction time (left turn situation)

Figure 5.5 ANN prediction for the perception-reaction time (departing minor road)

Figure 5.6 ANN prediction for diver's acceleration (left turn situation)

Figure 5.7 ANN prediction for diver's acceleration (departing minor road)

Figure 6.1 Flow chart for the validation code TWSC_VALID

Figure 6.2 Flow chart for the sensor-simulation code TWSC_SIM

Figure 6.3 Effect of range measuring precision on the bullet time error

Figure 6.4 Effect of range measuring precision on the safety margin error

Figure 6.5 Effect of azimuth angle measuring precision on the bullet time error

Figure 6.6 Effect of azimuth angle measuring precision on the safety margin error 
Figure 6.7 Effect of side offset and approaching speed on the reading error Figure 6.8 Effect of side offset on the critical distance for hidden vehicles

Figure 6.9 Effect of bullet vehicle acceleration model on computing bullet time Figure 6.10 Effect of bullet vehicle acceleration model on computing safety margin 


\section{LIST OF APPENDICES}

Appendix "A" Simulation Scenario for Left-Turn Situations

Appendix "B" Simulation Scenario for TWSC Situations

Appendix "C" MATLAB Code for Validating the Proposed System

Appendix “D” MATLAB Output Report for Validation Code

Appendix "E" MATLAB Code to Simulate Detection Sensor

Appendix "F" MATLAB Output Report for Sensor Simulation Code

Appendix "G" MATLAB Code for Constant Acceleration 


\section{CHAPTER 1: PROBLEM DEFENITION AND STUDY FRAMEWORK}

This chapter provides information about the research background, problem identification, objectives and scope of the research. A description is also provided regarding the study methodology used to achieve the research objectives.

\subsection{Introduction}

Road safety has direct impact on the wealth and welfare of any nation. It was found that approximately 1.2 million people are killed each and every year in traffic collisions throughout the world with many more millions seriously injured (Murray and Lopez, 1996; Dahl 2004). In 1990, road traffic injuries were ranked as the ninth leading contributor to the global burden of disease and injury. If proper measures are not introduced to curtail these trends they are expected to be ranked third by year 2020.

In-vehicle safety technologies are among the measures aimed at improving road safety by reducing traffic collisions and their severity. They could be either active safety measures that are intended to avoid collisions or passive safety measures that are intended to mitigate the severity of collisions when they occur. Vehicle-mounted collision warning and avoidance systems are among the most popular, yet important, technologies in the active safety measures. Those systems widely vary from a simple audible and/or visual warning to a more sophisticated intelligent system that adjusts 
steering, throttle and brakes to compensate for driver's error or delay in taking proper corrective actions (Drew 1994; Kiefer et al. 199; ENG 2001; Granet et al. 2003).

In-vehicle telematics are form of modern intelligent transportation systems (ITS). It should be noted that driving performance may be impaired by improper design and use of in-vehicle technologies (TC 2003; ITS America 2002). A study by the Texas Transportation Institute evaluated the impacts of ITS from a behavioural perspective (Lomax et al. 2002), where it was found that some of the in-vehicle technologies may lead to more risky behaviour, and that there is a need to reduce driver workload and fatigue through better design. As well, drivers alter their behaviour based on the conditions within their vehicle. For example, driver behaviour is influenced by the general handling characteristics, the existence of driver support systems (e.g., antilock brakes, cruise control, and information systems), field of view, comfort level (e.g. noise and vibration conditions, heating and air conditioning levels), and road lighting (Seiffert and Wech, 2003). Any control button or switch found on an information panel (dashboard) can take the attention of a driver away from driving and if sufficiently distracting, can potentially have the driver looking away from the road for extended periods of time.

Most in-vehicle collision warning and/or avoidance systems are designed to detect potential rear-end collisions (Brown et al. 2002; Taylor 2005; Dravidam and Tosunoglu 2000a). Less attention was paid to warning/avoidance systems that detect other types of collisions, such as left-turn or angle collisions. In Ontario - Canada, there were 22,600 angle collisions and 39,375 turning movement collisions in 2004 with a total of 61,975 collisions from which 147 were fatal and 15,514 caused injuries 
(MTO 2005). This is compared to 60,344 rear-end collisions from which 30 were fatal and 13,517 caused injuries. Similar comparisons for years 1999-2004 are shown in Table 1.1, which indicates that the angle or turning movement collisions are as frequent as rear-end collisions, while their severity is higher.

\subsection{Problem Definition}

As mentioned above, intersection collisions, including left-turn and angle collisions, cause more fatalities and injuries than rear-end collisions. Nevertheless, less attention was paid to them in the design of modern collision warning and/or avoidance systems.

Table 1.1 Different collision types in Ontario

\begin{tabular}{|c|c|c|c|c|c|c|}
\hline \multirow{2}{*}{ Year } & \multicolumn{3}{|c|}{ Angle + Turning Movement Collisions } & \multicolumn{3}{c|}{ Rear-end Collisions } \\
\cline { 2 - 7 } & Fatal & Injuries & Total & Fatal & Injuries & Total \\
\hline 1999 & 169 & 19,184 & 70,924 & 40 & 16,616 & 56,687 \\
\hline 2000 & 161 & 18,571 & 70,544 & 41 & 17,191 & 62,877 \\
\hline 2001 & 155 & 17,472 & 66,767 & 32 & 16,395 & 62,722 \\
\hline 2002 & 149 & 16,912 & 63,755 & 32 & 16,591 & 64,718 \\
\hline 2003 & 143 & 15,997 & 62,957 & 36 & 14,853 & 63,369 \\
\hline 2004 & 139 & 16,004 & 63,162 & 40 & 13,450 & 60,351 \\
\hline 2005 & 147 & 15,514 & 61,975 & 30 & 13,517 & 60,344 \\
\hline
\end{tabular}


Many of intersection collisions occur at situations where the driver has to select a proper gap to proceed into an intersection that is either non-signalised or signalised during the permitted left-turn phase. Left-turn collisions may occur at either non-signalised or signalised intersections as per one of the following two scenarios:

- At a non- signalised intersection, such as two-way stop controlled (TWSC) or two-way yield controlled (TWYC) intersections, where the driver of the target vehicle tries to make a left-turn from the major highway (uncontrolled) into the minor highway (controlled) while a bullet vehicle is approaching the intersection from the opposite side on the major uncontrolled highway, as shown in Figure 1.1(a); or

- At a signalised intersection where the driver of the target vehicle tries to make a left-turn from the major highway during a permitted phase while a bullet vehicle is approaching the intersection from the opposite side on the major highway, as shown in Figure 1.1(b).

Both scenarios above are identical in terms of perception-reaction of the drivers of either the target vehicle or the bullet vehicle. On the other hand, angle collisions that result from driver's improper gap selection may only occur at non-signalised intersections, specifically at a two-way-stop-controlled (TWSC) intersection, or at twoway-yield-controlled (TWYC) intersection. In either case, the driver of the target vehicle tries to exit the minor controlled roadway by making a right turn, a left turn or passing through the uncontrolled major roadway while a bullet vehicle is travelling on the major roadway, as shown in Figure 1.2(a). 
It should be noted that there are other types of angle collisions that are not related to driver's improper gap selection and they usually result from driver's violation of traffic by-laws. An example for that is at a signalised intersection where a driver tries to pass through an intersection while the traffic signal is in its red phase (usually at the start of the red phase), as shown in Figure 1.2(b). In this case, that vehicle would be either a target vehicle or a bullet vehicle, and any possible collision would be solely resulted from that driver's aggressive driving attitudes rather than from his/her misjudgement. This is based on the assumption that the cycle length of the traffic signal is properly designed and provides adequate amber phase that does not cause dilemma for the driver. Furthermore, most traffic agencies provide an all-red phase to reduce the probability of the occurrence of this type of collisions.

Based on the above, a collision warning system will not be useful in reducing that particular type of collisions that are based on driver's violation of the law; therefore there is no need for any collision warning system to address this situation because the driver is already informed by the red traffic signal and it is his/her aggressive driving attitudes, not the lack of information, that lead to this type of collisions. Proper law enforcement measures, such as red-light cameras, might be more effective to address this particular type of collisions.

The factors that affect the probability of an intersection collision to occur can be summarised as follows:

- Geometric characteristics of the roadway, including the number of lanes to be crossed, lane width, median width, slope, and the existence of an exclusive left-turn lane. 


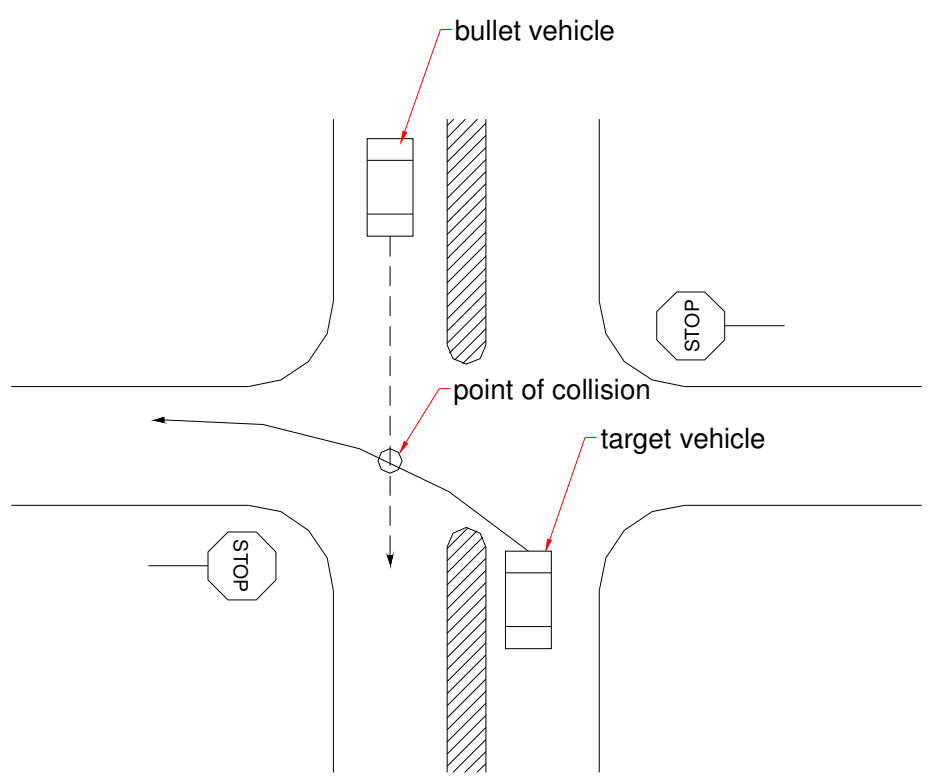

(a) Unsignalised intersection

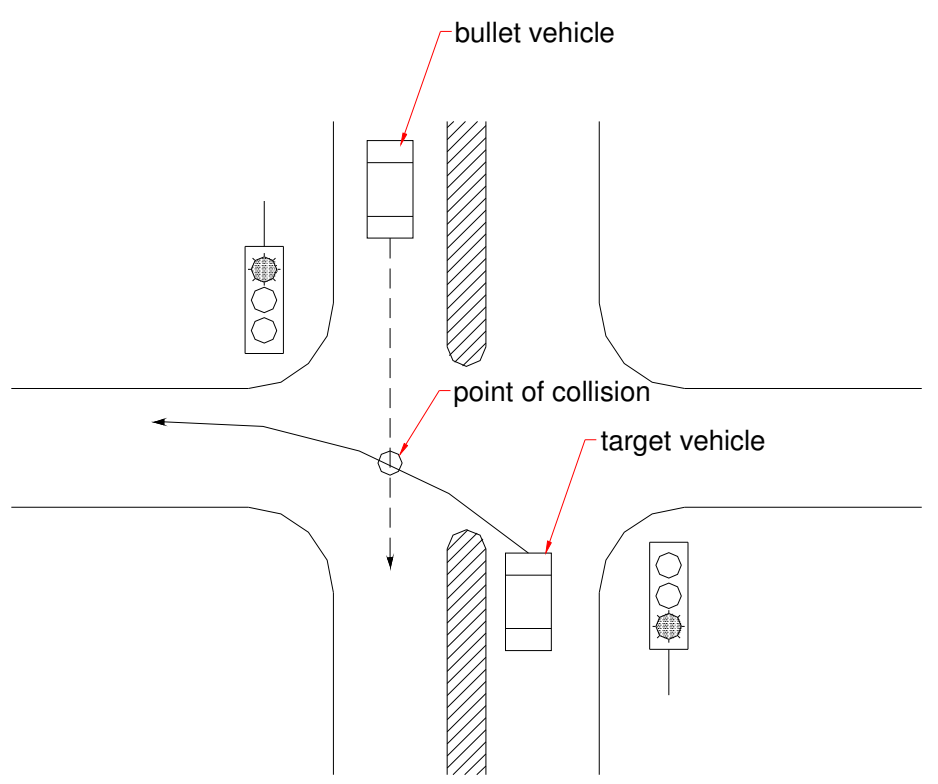

(b) Signalised intersection

Figure 1.1 Left-turn collisions at unsignalised and signalised intersections 


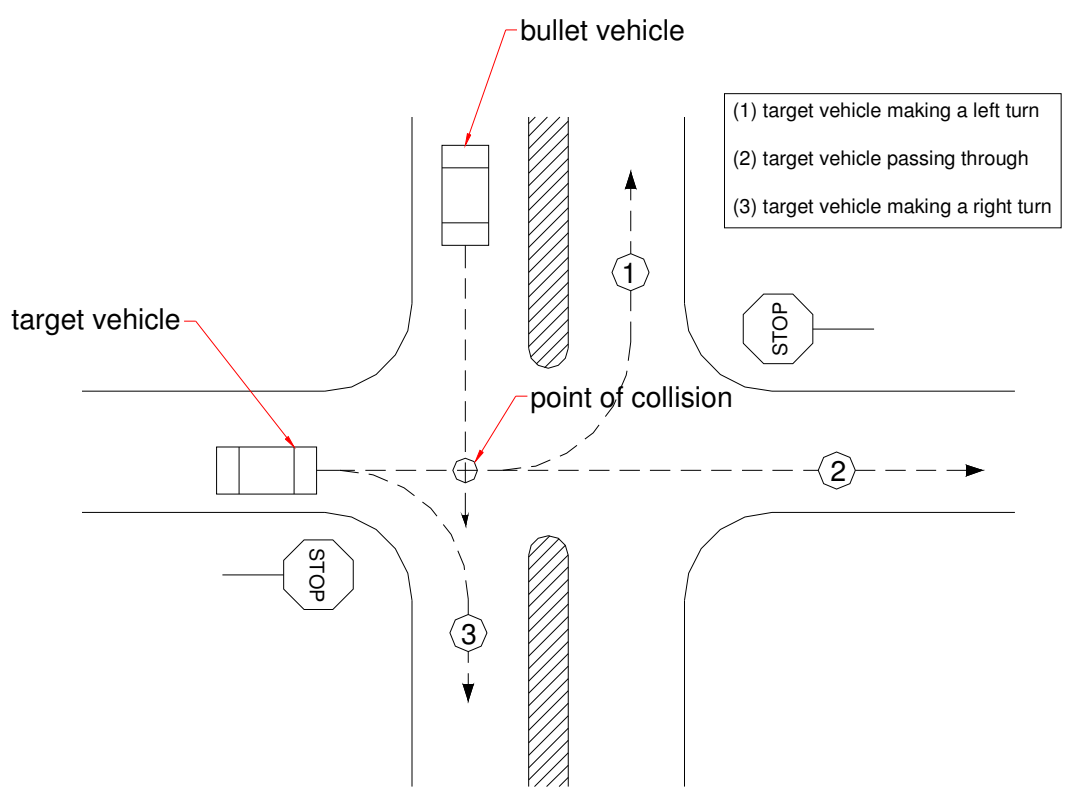

(a) Unsignalised intersection

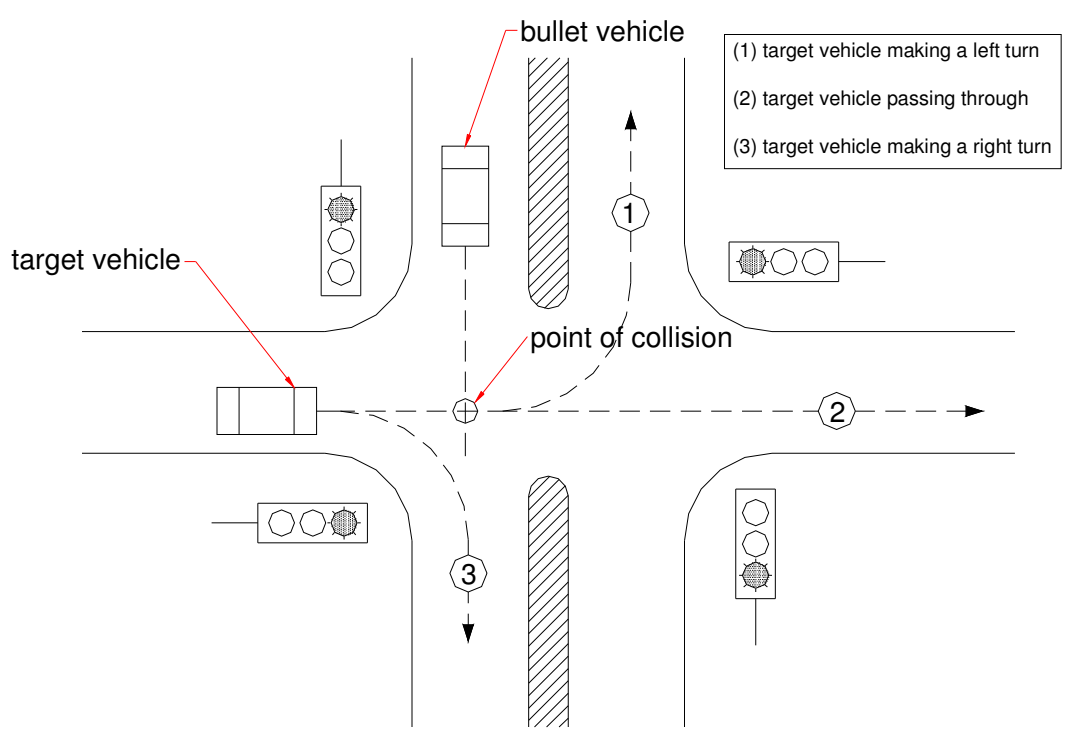

(b) Signalised intersection

Figure 1.2 Angle collisions at unsignalised and signalised intersections 
- Speed and acceleration/deceleration of the approaching bullet vehicle.

- Dynamic and physical characteristics of the target vehicle, including rate of acceleration and the length of the vehicle.

- Perception/reaction of the driver of the target vehicle, which is considered as the most important factor that leads to an imminent potential intersection collision. This can be described as the driver's ability to perceive all the factors stated above, analyse them, make appropriate decision, and perform the leftturn action.

One of the most leading factors that may lead to intersection collisions is the driver's misjudgement of oncoming/cross traffic gap, which resulted in approximately $36.1 \%$ of collisions at intersections (Pierowicz 1997; Jocoy and Pierowicz 1998; Pierowicz et al. 2000); and therefore, any warning system for intersection collisions should provide sufficient information for the driver about the speed, acceleration and time-to-collision for other vehicles in oncoming/cross traffic streams.

Although the human visual system is extremely sophisticated, psychophysical evidence found that it is insensitive to the acceleration of objects with no direct perceptual mechanisms to support the perception of acceleration (Watamaniuk and Duchon 1992). A properly-designed collision warning system might mitigate that problem by perceiving all the information, including driver's reaction time, analyzing them and giving a visual, auditory, or haptic signal to the driver to start proceeding into the intersection by allowing adequate time for the driver's perception to the signal, driver's reaction, and the time required to clear the intersection. 
A key factor in the success of any collision warning system is the consideration given for the driver's perception-reaction time, which is the time required for the driver to perceive the signal initiated by the system and react to it. There are many human factors that affect the perception-reaction time for any driver, including age, health conditions, driver's attitudes and his/her familiarity with the vehicle while driving.

When designing any in-vehicle collisions warning system, its benefits were found to depend on the joint performance of the system and the driver (Dravidam and Tosunoglu, 2000a). This means that many in-vehicle technologically sophisticated systems fail, not because of technical shortcomings, but because designers failed to consider the role of the human operator. As well, in order to be effective, drivers must accept in-vehicle technology. In general, the system must be useful to the driver and must not interfere with normal driving habits. The system should result in a minimum load on driver's attention.

Timing of triggering the alarm is a key factor in the success of any in-vehicle collision warning system. Unrealistic warnings may become a nuisance and lead to the loss of system's reliability for the driver. Hence, the performance should be improved in terms of reliability and optimal timing for providing the warning messages that give adequate time for the driver to perceive the message and take proper actions with minimizing unrealistic messages that might be ignored by the driver and therefore impact the reliability of the system. 


\subsection{Objectives and Scope}

The objectives of this research can be summarised as follows:

1. To develop a new collision warning system to assist drivers in selecting proper gaps at intersections, including the following two cases that are based on gap acceptance:

a. Making a left turn from a major road into another road in either a non-signalised intersection or a signalised intersection during the permitted left-turn phase; and

b. Departing a controlled minor road at a semi-controlled intersection.

2. To specify the minimum specifications for key hardware components.

3. To develop the required algorithms for different situations.

4. To ensure that the proposed system triggers the warning signal in realistic timing that does not cause nuisance to the driver of the equipped vehicle, the objective of this research includes also incorporating human factors into the proposed system to reduce or eliminate unnecessary warnings. The human factors that will be covered by this research include:

a. Estimating the perception-reaction time for the driver of the equipped vehicle, which is the time required to perceive the message given by the system and react to it by activating the throttle; and

b. Estimating the acceleration rate selected by the driver of the equipped vehicle when making the departure movement. 
To estimate the above human factors, two statistical techniques will be consequently implemented as follows:

a. The first technique is by using linear regression models at the start stage of implementing the proposed system; and

b. The second technique is to use more-sophisticated and self-learning Artificial Neural Network (ANN) models, which should be implemented once the system creates a database for actual human response as recorded by the system, and that database has an adequate size to produce Artificial Neural Network (ANN) models with mean-root-squared-errors that are less than that for the initial linear regression models.

5. Finally, the scope of this research also includes validating the proposed system by creating simulation scenarios, using MATLAB software, to simulate the proposed system and evaluate its reliability under different conditions and to assess the impact of different components on the functionality and reliability of the system.

It should be noted that the research scope does not cover the circuit design of the system or the provision of any information for building a prototype or offering the system as a commercial product. Further research and development will be required to achieve those goals, which are beyond the scope of this research and should be achieved using multi-disciplinary approach where other researchers should be involved from the fields of electrical and mechanical engineering. 


\subsection{Study Methodology}

To detail the tasks required to conduct the research, a flow chart has been created as shown in Figure 1.3. The tasks are shown below:

- Chapter 1 includes problem identification and definition of the various aspects of the problem that should be solved. It also includes the development of the methodological framework and identifying the resources required to achieve it.

- Chapter 2 includes literature review focusing on the following topics:

- Driver's errors and human factors at intersections;

- Existing collision warning/avoidance systems and identifying their benefits and limitations;

- Different mathematical models that describe vehicle dynamics and acceleration profile at different time instances, and

- The driving simulation technique used for the research and its advantages and features.

- Chapter 3 includes the algorithm design for detecting imminent left-turn collisions, including calculating the time required for each approaching vehicle to reach the intersection, calculating the time required for the departing vehicle to clear the path of the approaching vehicles, and the safety criterion used to decide whether or not a 'safe' message should be displayed to the driver of the departing vehicle.

- Chapter 4 includes the algorithm design for detecting imminent angle collisions, including calculating the time required for each approaching vehicle 
to reach the intersection, calculating the time required for the departing vehicle to clear the path of the approaching vehicles, and the safety criterion used to decide whether or not a 'safe' message should be displayed for the driver of the departing vehicle. The chapter covers the calculations of those times in different conflict situations - whether if the paths of the approaching and departing vehicles are perpendicular to each other, parallel to each other or located on the same lane.

- Chapter 5 includes the calibration and validation of the statistical models used to estimate human factors, including driver's perception-reaction time as well as driver's selection for the acceleration rate of the turning vehicle. It also covers in-vehicle model improvement for human factors using neural network analysis, which is based on actual response times and acceleration rates as recorded for different drivers at different situations.

- Chapter 6 includes system validation and sensitivity analysis using MATLAB.

- Chapter 7 includes summary, conclusions, limitations and recommendations for further research directions. 


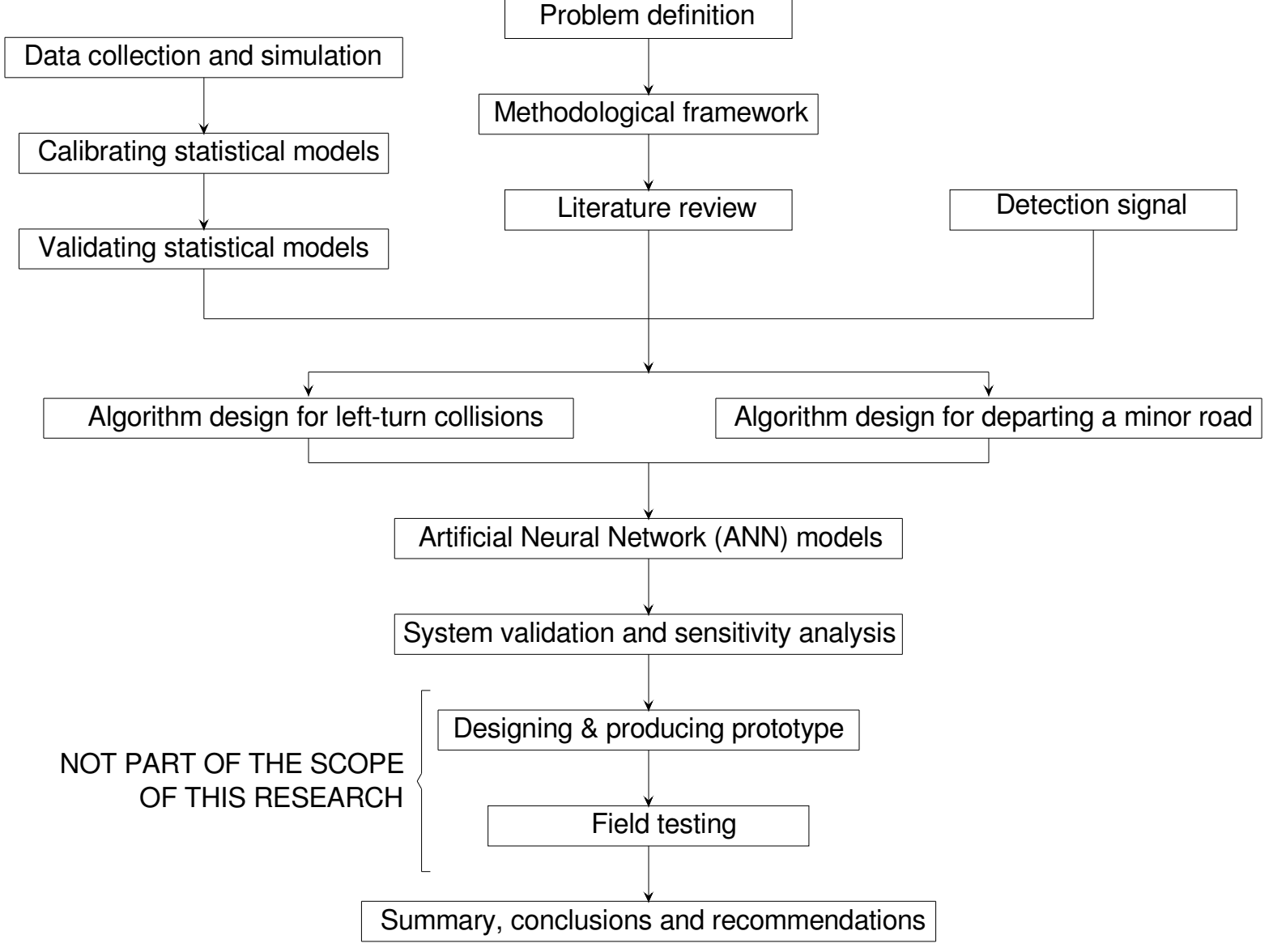

Figure 1.3 Flow chart for research methodology 


\section{CHAPTER 2: LITERATURE REVIEW}

This chapter focuses on human factors at intersections and different factors that might lead to intersection collisions. This chapter also focuses on the existing collision warning/avoidance systems and identifies their benefits and limitations and how driver-vehicle interaction and human factors at intersections should be considered when developing new algorithms for intersection collision warning systems. Vehicle dynamics and different models that describe vehicle acceleration are also covered. This chapter also covers the existing driving simulation techniques with emphasis on the technique used in this research. Existing methods used for vehicle tracking and vehicle-lane association are also discussed.

\subsection{Human Factors at Intersections}

The total time required for a driver to make a left turn or depart a controlled intersection is shown in Figure 2.1, which is the sum of the following times (AASHTO 2001; TAC 1999):

1. Driver's perception-reaction time $\left(t_{1}\right)$, which is the time required for the driver to perceive the situation, analyse it, make a decision and take appropriate action by activating the throttle; and

2. Vehicle's travel time $\left(t_{2}\right)$, which is the time required to accelerate the vehicle and to clear the intersection. This includes the time required to pass a total 
distance that equals to the sum of the offset distance between the approaching and departing vehicles plus the length of the departing vehicle itself.

To achieve a successful safe departure, the total time required for a target vehicle to depart the intersection should be less than the time required for an approaching bullet vehicle to reach the intersection from the moment the driver starts to perceive the situation $\left(t_{\text {bullet }}\right)$. A safe departure is ensured only if the following criterion is met:

$$
t_{1}+t_{2}<t_{\text {Bullet }}
$$

A collision may occur if the driver of the departing vehicle underestimates his/her vehicle's travel time $\left(t_{2}\right)$ or overestimates the time required for the bullet vehicle to reach the intersection $\left(t_{\text {bullet }}\right)$. A collision may also occur if the driver's perceptionreaction time exceeds the difference between $t_{\text {bullet }}$ and $t_{2}$.

Based on the above analysis, an intersection collision mainly results from a human error. This fact was confirmed by earlier research (Pierowicz et al, 2000), where it was found that nearly $75 \%$ of all intersection collisions were due to driver error with the following main factors that compose a driver error:

○ Driver Inattention: $28.7 \%$;

○ Faulty Perception: $33.9 \%$; and

- Vision Obstruction: $11.1 \%$ 


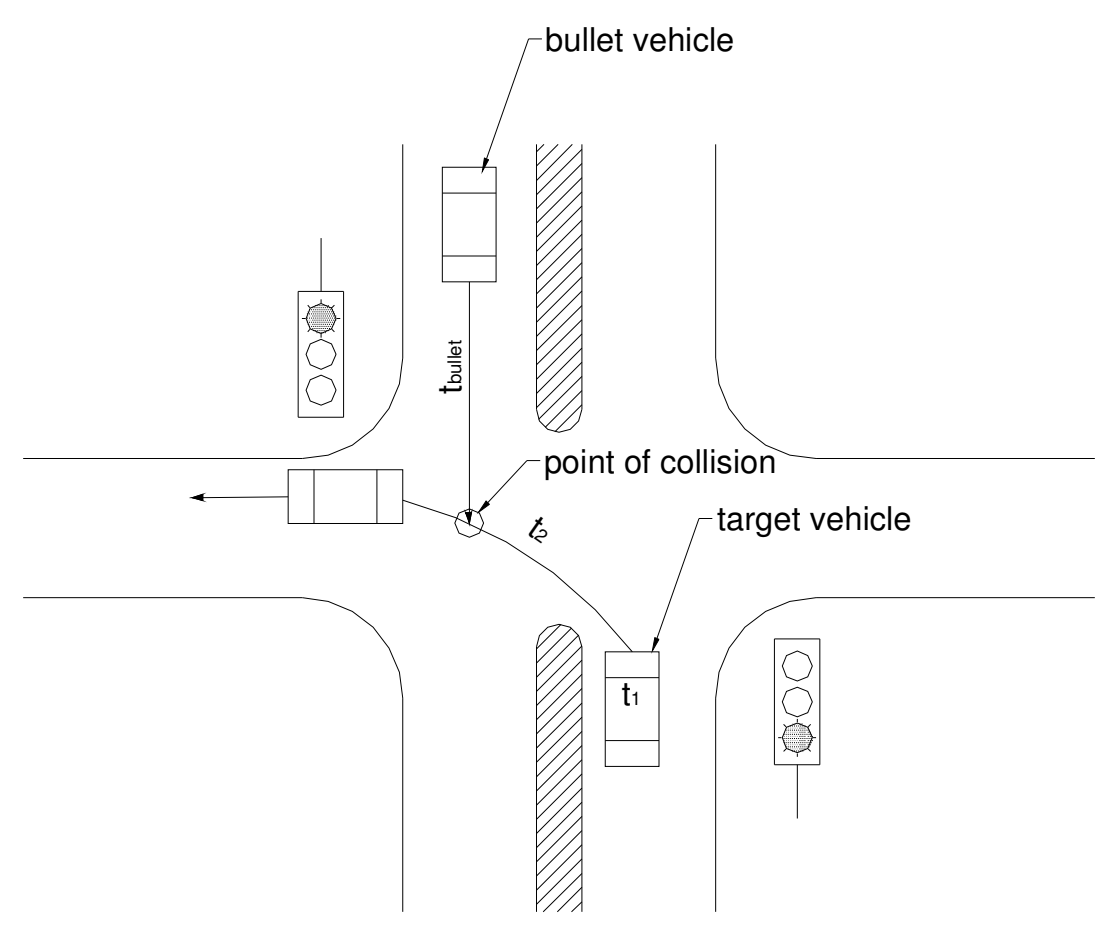

Figure 2.1 Time criterion for left-turn collisions

In another study on all crashes in the United States for the year 1993 (Najm et al 1995) that was supported by the National Highway Traffic Safety Administration (NHTSA), it was found that $48.5 \%$ of left-turn crashes were due to driver's recognition error, and $40.9 \%$ were due to driver's decision error. Vehicle and road conditions were found to have no significant effect on this type of crashes. This finding can be explained in the light of the fact that a driver's proper reaction should be based on his/her perception for vehicle and road conditions.

Both younger and older drivers may be involved in intersection collisions. Older drivers have slower perception-reaction times and exhibit more difficulty in selecting safe gaps and judging speeds of oncoming vehicles than younger drivers. It was 
found that $19 \%$ of older drivers found that making a left turn was getting more difficult as they grow older (FHWA 2001). On the other hand, younger drivers may also be involved in intersection collisions due to their lack of experience and/or risky driving behaviour. It was found that the youngest age group, less than 24 years old, is the most likely to be involved in a left-turn collision during permitted phase (CTRE 2004).

A major cause for driver's misjudgement or faulty perception is when the oncoming bullet vehicle is accelerating. One of the most common reasons for the bullet vehicle to accelerate is when the driver of the bullet vehicle tries to pass a signalised intersection near the end of the green phase. As was mentioned earlier, the problem with acceleration is that the human vision system is insensitive to the acceleration of objects with no direct perceptual mechanisms to support the perception of acceleration (Watamaniuk and Duchon, 1992).

The above findings indicate that a countermeasure to mitigate or reduce driver errors, such as a driver warning system, would significantly reduce the likelihood of intersection collisions. If a target vehicle is equipped with a proper intersection collision warning system, the driver's mental load will be significantly reduced as it will be only limited to perceiving the warning signal and reacting to it instead of perceiving an entire situation. However, such a warning system should be proven reliable to the driver in order to minimize perception-reaction time, which will vary from one driver to another. 


\subsection{Existing Collision Warning Systems}

All existing collision warning systems are developed to address rear-end collisions with few of them can also address side-swipe collisions to some extent (VORAD 2009). Those systems incorporate either radar-based or laser-based (LIDAR) sensors. As the technology used to develop those systems improved, so did the accuracy and, in some cases, complexity of the algorithms used to trigger a warning to the driver and/or an automatic vehicle response.

A collision warning system generally operates by using a sensor installed at the front bumper of a vehicle that constantly scans the road ahead for vehicles or obstacles. When found, the system determines whether the vehicle is in imminent danger of colliding, and if so, warns the driver and/or takes the proper corrective action either by automatic braking or steering. The sensor systems employed can measure clearance and relative velocity of the lead vehicle and the acceleration is estimated through the coordinated systems using a radio to transmit the lead car velocity and acceleration data (Drew 1994).

Several collision warning systems have been developed by different auto makers, research institutes, and government agencies. In general, the algorithms can be classified according to their use of time-headway, time-to-collision, or the underlying kinematics constraints. The time-headway algorithms offer simplicity and are indirectly influenced by relative velocity. They have been designed to be consistent with current manual recommendations for safe driving (i.e. 2-sec gap). Time-tocollision algorithms are based on perception of contact time and are sensitive to 
relative velocity. Algorithms based on kinematics constraints offer increased accuracy by calculating the moment that the driver must initiate braking, given an assumed reaction time and following vehicle deceleration response. Because this class of algorithm considers both the reaction time and the capacity of the following vehicle to decelerate, it offers more comprehensive model than the other two categories.

Most of the new generation of assistance devices are derivatives, in one form or another, of the adaptive cruise-control system (Smith 2000; ENG 2001). They work by detecting the distance and velocity of the vehicle ahead. The easiest way to do this is to use a small onboard sensor system (ENG 2001; Granet et al. 2003). These systems use different optical, electromagnetic radar, or ultrasonic techniques. Researchers are considering combinations of several of these technologies (sensor fusion), to overcome some of the disadvantages (Smith 2000; Granet et al. 2003).

Overall, these systems measure the distance between the two vehicles, to within a few centimetres, using the time that it takes for signals of the sensor beam to travel to the vehicle ahead and bounce back (Granet et al. 2003). On the other hand, the velocity of the vehicle ahead is determined by measuring how the Doppler Effect has altered the frequency of the radar beam that has echoed back. The difference in frequency between the transmitted and received signals gives a measure of how much faster or slower the vehicle ahead is travelling (ENG 2001). In some cases, a small digital camera keeps an eye on the road ahead, processing continuously to track the lane marking. Such examples are present on the Fiat, Nissan, DaimlerChrysler, Volvo, BMW, Subaru, Honda, and Mitsubishi (ENG 2001). Each specific 
vehicle may have slightly different features and functionalities. The core of the system is the collision avoidance algorithm, which receives the inputs from the sensor suite (active and vehicle sensors), processes them and provides the appropriate driver-vehicle warning response.

In 1999, the Chinese Academy of Sciences' Intelligent Control and Systems Engineering Centre started the Intelligent Vehicle Platforms project. Supported by the Vehicular Embedded Computing Platform project, the Xi'an Jiaotong University Institute of Artificial Intelligence and Robotics collaborated to develop intelligent driver-assistance and safety warning systems for passenger vehicles, particularly GPS- and vision-based systems (Zheng et al. 2004). Those systems provide appropriate real time information regarding the vehicle, road, and surroundings for safer and better driving. The system architecture contains three layers: the sensory layer, decision-making layer, and the human-interface layer (Zheng et al. 2004). It warns the driver passively when his or her vehicle is in a hazardous situation and proactively about possible hazardous situations on the basis of the vehicle's current position, orientation, and velocity, and the road conditions or surrounding environment.

\subsection{Intersection Collision Warning/Avoidance Systems}

As stated above, all commercially available collision warning systems are mainly designed for rear-end and/or for lane-changing collisions. However, there were some earlier research efforts to propose intersection collision warning systems. In 1996, 
Calspan SRL Corporation (later changed to Veridian Engineering) was awarded a 3year research contract from the United States National Highway Traffic Safety Administration (NHTSA) to develop a concept for intersection collision avoidance countermeasure (Pierowicz 1997; Jocoy and Phoel 1997; Jocoy and Pierowicz 1998; Pierowicz et al. 2000). That research program, named the Intersection Collision Avoidance (ICA) program, proposed fusing in-vehicle positioning system with radar sensors and communication system to detect potential violation of traffic control device, or proceeding into an intersection with inadequate gap, and to warn the driver accordingly by giving a warning message via a head-up display and haptic feedback. The program also proposed active control of vehicle's brake and steering to assist the driver in pre-crash situations.

That program only focused on detecting the speeds of the vehicles approaching the intersection without having specific algorithm to determine their acceleration/deceleration or time-to-collision and compare it to the time required for the equipped vehicle to clear the intersection. The program assumed that all approaching vehicles, as well as equipped vehicle, are moving in constant speeds. The program also did not give any consideration for the time required for the driver of the equipped vehicle to perceive the warning message and react to it. To detect the vehicles that may be in a collision path with the equipped vehicle, the program proposed the use of two radar sensors with $+/-90$ degree opening angles to either side of vehicle's longitudinal axis.

The proposed exceptionally wide opening angles might lead to unnecessary warnings when detecting vehicles that are not in a collision path with the equipped 
vehicle, which may dramatically impact the reliability of the system. Furthermore, most radar sensors available in the market have a maximum opening angle of 15-25 degrees; and therefore, designing a radar system with +/- 90 degree opening angle might be exceedingly costly and technologically challenging. To overcome this problem, the research project utilized $77 \mathrm{GHz}$ (later replaced with $24 \mathrm{GHz}$ ) sensors with rotating antennae. The use of rotating antenna led to technical challenges in synchronizing the rotating speed of the antenna with the update rate of the radar beam. It also compromised the accuracy in measuring both range and speed (using Doppler Effect) in addition to the difficulty in implementing the technology for commercial use.

The Automotive Collision Avoidance Systems (ACAS) program was launched in the United States in 1995 with its activities carried out by a consortium made up of government agencies as well as industrial and academic participants (NHTSA 2000). The goal of that program was to develop a comprehensive collision warning system with the capability of detecting and warning the driver of potential hazard conditions in the forward, side, and rear regions of the vehicle. The program proposed the use of long-range radar sensors to detect potential hazards in the front of the vehicle, along with short-range sensors to warn the driver of nearby objects when changing traffic lanes or backing up. Unlike the ICA program, the ACAS program effort was focused on providing warnings to the driver, rather than take active control of the vehicle.

In 1999, the United States Federal Highway Administration sponsored an intersection collision warning system (ICWS) project under the national Intelligent 
Transportation Systems program (FHWA 1999). The system comprises two types of traffic-actuated warning signs linked to pavement loops and a traffic signal controller. It was meant to enhance driver awareness of the traffic situation at intersections by providing warnings for drivers in case if a cross-traffic vehicle approaching the intersection, either from the controlled approach or the non-controlled approach.

The above system was mainly intended to compensate for inadequate intersection sight distance and therefore it only informs the driver of the presence of other cross-traffic vehicles without providing any further information about their speeds or acceleration rates. This might be suitable for intersections with extremely low traffic volume, but as the volume increases it will be necessary for the driver on the minor non-controlled road to depart the intersection even with other cross-traffic vehicles approaching the intersection. In this case, the driver will need more information about the approaching cross-traffic vehicles in order to make a proper decision to depart the intersection without compromising safety. The information needed about the cross-traffic vehicles includes their speed, acceleration and the anticipated time to reach the intersection. That system fails to provide such vital information.

In 2000, the Virginia Tech Transportation Institute (VTTI) was awarded a research contract from NHTSA to develop performance specifications and supporting objective tests for a field test of a vehicle-based countermeasure to intersection crashes (NHTSA 2004). The study targeted intersection crashes associated with violations of stop signs and traffic signals (red lights). The study was technology-independent that envisioned a warning system to warn drivers if they are in imminent danger of 
running a stop sign or signal, and is called the Intersection Crash Avoidance, Violation warning (ICAV) system. Similar to the preceding ICA system, the ICAV system also proposes the fusion of in-vehicle positioning system with radar sensors and communication system to detect potential violation of traffic control device, or proceeding into an intersection with inadequate gap.

As the ICAV system study program was technology-independent, it did not give particular specifications about the radar sensors proposed. The study also did not give particular algorithm for the TWSC situation; however, similar to the preceding ICA system, the ICAV system also assumes that the equipped vehicle is moving (not stopping) in violation of the traffic control device by-law and therefore the proposed algorithm would trigger a warning only if a cross traffic vehicle is expected to collide with the equipped vehicle that is traveling in a constant speed and/or acceleration (i.e., does not stop at the TWSC intersection).

White and Eccles (2002) proposed an infrastructure-based intersection collisionavoidance system (ICAS) designed to provide guidance for left-turning vehicles that have secondary right-of-way and are attempting a turn across the path of oppositedirection traffic. That system compares the presumed arrival time of the oppositedirection vehicle with the presumed time for a motorist to turn left. Based on this comparison, the system provides guidance to the left-turning motorist in the form of a dynamic sign placed in the same line of sight of opposite-direction traffic, so that motorists will have a "second opinion" to aid in their left-turn decisions.

The above system is composed of four modules: (a) a left-turn assistance device, (b) left-turn presence detection, (c) opposite-direction detection, and (d) a system 
processor. As there is a large difference between passenger cars and heavy vehicles in terms of acceleration, driver's eye height, and the length of the vehicle, the system differentiates between left-turning passenger cars and left-turning heavy vehicles and tailors its guidance appropriately. The algorithm is designed to undertake the following tasks for each left-turning vehicle:

- estimating the time required for left turn for each vehicle prior to making the left turn;

- determining the time estimated for the nearest oncoming vehicle to reach the intersection;

- comparing the two times; and

- indicating the result of the comparison to the left-turning driver in the form of an easily recognizable and understandable message displayed by a device that is in the line of sight for the left-turning driver.

In addition to the liability issues that might arise by the use of an infrastructurebased device, the above system has some other limitations. In order to accurately estimate the time required for a specific vehicle to make a left turn, the system should collect data about the vehicle, including vehicle classification, acceleration, and kinematics performance. The system should also collect data about the driver making the left turn, including age, perception-reaction time, and behavioural habits. The majority of these required data cannot be available for an infrastructure-based system and therefore the system presumes average values on the basis of ranges developed by prior research. 
In Europe, the European Commission (EC) launched the INTERSAFE research project in 2004 to generate a European approach to increase safety at intersections (Fuerstenberg 2005; Fuerstenberg and Chen 2007). The partners in the INTERSAFE project included vehicle manufacturers (BMW; VW; PSA; RENAULT), automotive suppliers (TRW; IBEO) as well as research institutes (INRIA; ika/FCS; Signalbau Huber). The project proposed the fusion of different technologies, including laser scanners, video camera, GPS and digital mapping, in addition to vehicle-toinfrastructure communication modules. The video camera was used to recognize the lane marking, along with data from the laser scanners and the GPS/digital map, for precise positioning of the equipped vehicle. The laser scanners, installed at the left and right front corners of the equipped vehicle, were also used for object detection, tracking, and classification.

The INTERSAFE system uses an algorithm that identifies all possible conflicts between the equipped vehicle and all detected vehicles using data from high-level digital map. It triggers a warning if the path of the equipped vehicle conflicts with the path of any of the detected vehicles assuming that all vehicles are moving. A probabilistic model for the behavior of all vehicles was deployed to determine the behaviour of the drivers of detected vehicles.

The INTERSAFE system proposed the use of continuous HMI for the drivervehicle interface (DVI). This means that at any time, the driver would have direct link to the risk level computed by the system so that the driver would be prepared for any risky situation and can use his/her own judgment for the intended maneuver by taking his/her own driving skills into account. 
Although the final report for the INTERSAFE project has not been published yet, it is evident that the major limitation of the proposed system is that it relies on many technologies (laser scanners, video camera, GPS, high-level digital map, vehicle-toinfrastructure communications), which may result in increased costs and other technical challenges. The algorithm used for the system assumes that all vehicles (including the equipped vehicle) are moving and it does not consider the time required for the driver to perceive the message given by the system and react to it.

A comparison is shown in Table 2.1 to illustrate the key features of previous major research projects related to collision warning systems, including the Intersection Collision Avoidance System (ICA), the Intersection Crash Avoidance, Violation System (ICAV), and the European Commission project (INTERSAFE) as well as the proposed system.

Some jurisdictions implemented another infrastructure-based collision prevention system at signalised intersections by simply offsetting the signal phasing of the leftturning and through motorists, such that the left-turning motorist has extra amber time remaining while the through motorists' phase is red (US DOT, 2003). The dynamic left-turn phase offset (DLTPO) scenario is accomplished by extending the left-turning motorist's amber phase a short but significant time in excess of the through vehicle's amber ball, when specific conditions warrant. The conditions warranting an offset are as follows:

- The end of the amber phase is approaching;

- A subject left-turning vehicle is detected in the intersection; 
Table 2.1 Comparison for different intersection collision warning systems

\begin{tabular}{|c|c|c|}
\hline System & Features & References \\
\hline $\begin{array}{l}\text { Intersection } \\
\text { Collision } \\
\text { Avoidance System } \\
\text { (ICA) }\end{array}$ & $\begin{array}{l}\text { 1. Utilized a pair of radar sensors and in-vehicle } \\
\text { positioning system. } \\
\text { 2. Assumed that all vehicles were moving in constant } \\
\text { speeds. } \\
\text { 3. No consideration given for the time required for } \\
\text { the turning driver to perceive the message given by } \\
\text { the system and react to it. }\end{array}$ & $\begin{array}{ll}\text { 1. } & \text { Pierowicz } 1997 \\
\text { 2. } & \text { Jocoy and Phoel } 1997 \\
\text { 3. } & \text { Jocoy and Pierowicz } \\
& 1998 \\
\text { 4. } & \text { Pierowicz et al. } 2000\end{array}$ \\
\hline $\begin{array}{l}\text { Intersection } \text { Crash } \\
\text { Avoidance, } \\
\text { Violation } \\
\text { (ICAV) }\end{array}$ & $\begin{array}{l}\text { 1. Technology-independent study (proposed the use } \\
\text { of a pair of radar sensors along with in-vehicle } \\
\text { positioning system). } \\
\text { 2. Assumed that the equipped vehicle was moving in } \\
\text { violation for traffic control device. } \\
\text { 3. No consideration given for the time required for } \\
\text { the turning driver to perceive the message given by } \\
\text { the system and react to it. }\end{array}$ & 1. NHTSA 2004 \\
\hline INTERSAFE & $\begin{array}{l}\text { 1. Utilized a pair of laser scanners, a video camera, } \\
\text { GPS and digital mapping, in addition to vehicle-to- } \\
\text { infrastructure communication modules. } \\
\text { 2. Assumed that all vehicles were moving and used } \\
\text { probabilistic model for the behavior of all vehicles. } \\
\text { 3. Utilized continuous HMI for the driver-vehicle } \\
\text { interface, which means that at any time, the driver } \\
\text { would have direct link to the risk level computed } \\
\text { by the system. } \\
\text { 4. No consideration given for the time required for } \\
\text { the turning driver to perceive the message given by } \\
\text { the system and react to it. }\end{array}$ & $\begin{array}{l}\text { 1. Fuerstenberg } 2005 \text { and } \\
\text { 2. Fuerstenberg and } \\
\text { Chen } 2007\end{array}$ \\
\hline Proposed system & $\begin{array}{l}\text { 2. Technology-independent study (proposes the } \\
\text { utilization of a pair of radar sensors or laser } \\
\text { scanners). } \\
\text { 3. Assumes that the equipped vehicle is starting from } \\
\text { stop (as in a typical semi-controlled intersection). } \\
\text { 4. Assumes that other vehicles are moving with } \\
\text { different speeds and acceleration rates. } \\
\text { 5. Gives consideration for the time required for the } \\
\text { turning driver to perceive the message given by the } \\
\text { system and react to it, and also gives consideration } \\
\text { for the variations among drivers in selecting their } \\
\text { desired acceleration rates when departing the } \\
\text { intersection. } \\
\text { 6. Gives consideration for the number of lanes to be } \\
\text { crossed by the equipped vehicle, including lanes } \\
\text { that may have vehicles traveling on them and not } \\
\text { detected by the sensors. }\end{array}$ & \\
\hline
\end{tabular}


- Opposite direction through traffic is present such that there is potential for a conflict; and

- No opposite direction left-turning motorists are present.

The above system is classified as a 'collision prevention system'. One of the limitations for that system is that it is only applicable at signalised intersections. It also has the same limitation as of any other infrastructure-based system, which are the assumptions of vehicle and driver average conditions. In addition, the system loses relevancy if the subject left-turning motorists anticipate the end of their amber before it is extended, not knowing that additional clear time exists. Conversely, if subject through vehicles repeatedly receive the extended amber light (due to frequent DLPTO activation), they may anticipate having a longer amber phase, making them liable to running red signals under normal signal conditions (when conditions do not warrant DLPTO).

There are many other collision prevention, or avoidance, systems applied by different jurisdictions at signalised intersections, including:

- Violator warning system, which detects a red-light violator upstream of an intersection, and provides a warning with enough time so that the motorist can stop the vehicle safely. The system is designed to differentiating, as earlier as possible, stopping vehicles from would-be violators.

- Dynamic extended red phase, which detects red-light violators that cannot stop, even with giving a warning, and extends the red phase for the cross traffic signal. 
- Cross traffic warning system, which provides a final opportunity to prevent a collision caused by a mid-cycle red-light violation. Warnings to right of way motorists can be applied at any time during the green/amber phase for the approach.

All the above systems are classified as collision prevention, or avoidance, systems and they are only applicable at signalised intersections. Moreover, all of them have the same limitations of infrastructure-based systems by assuming vehicle and driver average conditions.

In Japan, another collision warning system has been developed that combines the use of roadside infrastructure and in-vehicle sensor technology to assist or automate a driver's decisions in numerous potentially hazardous crash conditions at intersections (US DOT, 2003). The system utilizes road surface detectors; aboveground pedestrian/vehicle detectors; starting point markers; and two-way roadside/vehicle communication stations. The starting point markers are short-wave band $(13.56 \mathrm{MHz})$ emitters embedded in the road surface. In conjunction with an onvehicle receiving antenna and processing unit, it functions as a passage detector. Data input to the AHS would come from five sources:

- Stored vehicle-specific data (braking ability, vehicle type, etc.);

- Variable subject vehicle data (speed, position) generated both in-vehicle and through in-ground detectors;

- Variable principal other vehicle data (speed, position) generated from roadside detectors; 
- Stored road data (shape, intersection dimensions, lateral and longitudinal grade); and

- Variable road data about the surface condition of the road (i.e. wet, dry, or icy).

The above system has the same limitation of other infrastructure-based systems that it assumes critical variables related to driver behaviour, including perception reaction time (PRT), normal deceleration, and driver behaviour. The system assumes a driver response to information time of 2.65 seconds. The assumed driver response time to a warning is 1.0 seconds. The assumed normal deceleration rate is $0.3 \mathrm{~g}$, and emergency braking is assumed to be $0.5 \mathrm{~g}$. Also, these values are in conjunction with a flat, straight, wet road (with a minimum coefficient of friction value

of 0.5 ). The times were chosen to cover 90 percent of motorists (70 percent of elderly drivers). The roadside communication is by two-way radio transmission operating at $5.8 \mathrm{GHz}$ and covering a transmission zone of 100 meters.

\subsection{Vehicle Acceleration Models}

A key issue in designing any collision warning system is to select the proper acceleration profile that is used to estimate the anticipated times for the approaching vehicles to reach the intersection as well as the time required for the equipped vehicle to clear the paths of the approaching vehicles. The maximum acceleration that can be provided by a vehicle is based on its size, weight and engine power. There are non-driver-related factors that may reduce the maximum acceleration 
provided by a vehicle; including grade resistance, aerodynamic resistance and rolling resistance (Mannering et al. 2005). Furthermore, drivers usually select an acceleration rate that is less than the maximum acceleration provided by the mechanical characteristics of the vehicle and it depends on other factors related to driver's personality and his/her perception for the situation (e.g., the location and the speed of the nearest approaching vehicle). Different models were developed to represent the time-acceleration profile. The simplest model is by assuming a constant acceleration by using the following simple equations:

$$
\begin{aligned}
& a=\text { constant } \\
& v=v_{0}+a t \\
& d=d_{0}+v_{0} t+1 / 2 a t^{2}
\end{aligned}
$$

where $a$ is the constant acceleration rate (in the distance / time / time units), $v$ is the speed of the vehicle at time $t$ (in distance / time units), $v_{0}$ is the initial speed of the vehicle (in distance / time units) and $d$ is the distance traveled by the vehicle at time $t$ (in distance units). This constant acceleration model may not be realistic reflection of vehicle acceleration as it was found that there is a linear relationship between vehicle acceleration and its speed where vehicles accelerate more at lower speeds and less at higher speeds as shown in Figure 2.2 (Drew 1968; Long 2000). This linear relationship can be represented by the following set of equations: 


$$
\begin{aligned}
& \frac{d v}{d t}=\alpha-\beta v \\
& v=\frac{\alpha}{\beta}-\left(\frac{\alpha}{\beta}-v_{0}\right) e^{-\beta t} \\
& d=\frac{\alpha}{\beta} t-\left(\frac{\alpha}{\beta}-v_{0}\right) \frac{\left(1-e^{-\beta t}\right)}{\beta}
\end{aligned}
$$

where $\alpha$ and $\beta$ are model parameters that are based on the mechanical characteristics of the vehicle where $\alpha$ represents the maximum acceleration at the start of the departure movement, and $\beta$ represents the rate of decrease in acceleration as the speed, $v$, increases.

The decreasing linear relationship between acceleration and vehicle speed has been reported in a number of studies. In a detailed investigation of the effect of grades on traffic movements, test results found in the literature have been examined and validated by newer tests (St. John and Kobett 1978) where it was found that the acceleration capability of passenger cars, pickup trucks, and recreational vehicles was a linearly decreasing function of speed. That study found a linear relationship with $\alpha=3.36$ and $\beta=0.0803$ for a 1970 Chevrolet sedan on level terrain, and $\alpha=$ 5.19 and $\beta=0.1478$ for a 1971 Chevrolet three-quarter-ton pickup truck on level terrain, under maximum acceleration.

Similar results have been also provided for other vehicles (Glauz et al. 1980) where a linear relationship was also found with $\alpha=2.5$ and $\beta=0.104$ for the acceleration characteristics of the lowest 10 percent in performance capabilities of recreational vehicles, and $\alpha=2.85$ and $\beta=0.0853$ for passenger cars. 
In 1958, traffic data have been collected at three signalized intersections in New Jersey (Bellis 1960) where the data for passenger cars were too incomplete to be useful, but the truck data had fewer gaps and exhibited a linearly decreasing relationship with $\alpha=1.67$ and $\beta=0.1229$. The truck in that early study may be closer to SU (single unit) vehicles in the current design guides.

Another study in 1966 (Dockerty 1966) used motion picture photography to study the acceleration characteristics of leading vehicles queued at two traffic signals in England to capture actual motorist behavior in traffic streams. The distances of movement have been measured during 1-s intervals, commencing at the beginning of vehicle motion.

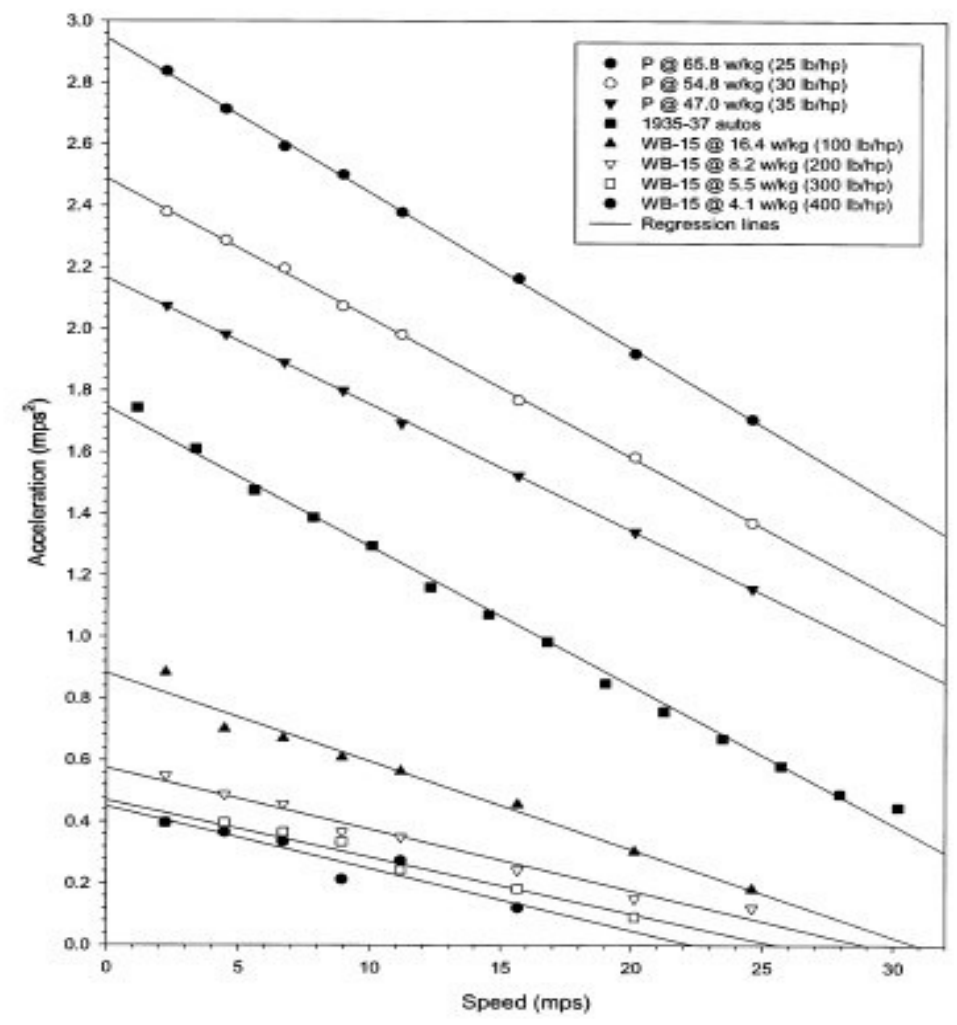

Figure 2.2 Maximum acceleration for different vehicles [Long 2000] 
Observations were separated for passenger cars, small vans, and medium vans from heavy vehicles, which included all others. Although that study did not present it, a linearly decreasing model with $\alpha=2.27$ and $\beta=0.1487$ fits the data from those observations of cars starting in the median lane. Those data reflect that leading vehicles tend to accelerate in the outside travel lane a little slower, with $\alpha=1.88$ and $\beta=0.1201$.

In 1972, another study (Evans and Rothery 1972) collected speed and acceleration data on the characteristics of 15,138 queued motorists crossing a trap after stopping for a red traffic signal at a suburban location near Detroit. From that data, a different study (Bonneson 1992) calculated a strong linearly decreasing relationship between acceleration and speed. In that same study more data were collected using the same technique of the older to collect acceleration and speed data for a sample of 4,820 passenger cars accelerating straight ahead at five different signalized sites in two states. The data showed very strong linear relationships between acceleration and speed, with $\alpha=2.02$ for each traffic stream. The five through-traffic streams had very similar accelerations, ranging from $\beta=$ 0.1184 to $\beta=0.1326$.

In another treatise (Bonneson 1990), data were collected on eight left-turning movements with a total sample size of 13,881 passenger cars where a linear relationship was also found with $\alpha=2.02$. All left-turning movements showed slower accelerations at higher speeds than through movements, ranging from $\beta=0.1339$ to $\beta=0.3157$, probably due to centrifugal forces affecting occupant comfort while turning through different radii under normal conditions. 
Acceleration data for a sample of 98 left-turning and 342 right-turning vehicles moving from minor streets into cross-street gaps of $10 \mathrm{~s}$ or less were reported in another study (Harwood et al. 1996). This limitation on gap time was imposed to select samples wherein drivers had some incentive to use relatively high accelerations. Speeds were measured across a series of four $61-\mathrm{m}$ to $122-\mathrm{m}$ (200-ft to $400-\mathrm{ft}$ ) traps on the cross streets, so the observations did not provide much detail, did not provide startup accelerations, and were merely summarized in the report. All the computations assumed that each vehicle had a constant acceleration over each long trap.

Another study (Wang et al. 2004) used database generated by 100 personal vehicles in Atlanta urban area equipped with Global Positioning System (GPS), accelerometers, and brake sensors. Vehicle speeds were measured every 1 second using the GPS via wireless connection. The data collected included data from vehicles moving straight as well as from turning vehicles. For vehicles moving straight, a linear relationship was found with $\alpha=1.883$ and $\beta=0.021$. For turning vehicles, a linear relationship was also found with $\alpha=1.646$ and $\beta=0.017$.

A polynomial acceleration model was introduced in another study (Akcelik and Biggs 1987) where speeds were measured in 1-second intervals for 1,037 vehicles accelerating from a stop in three different locations (central business district, urban, and non-urban) in Australia, collected with an instrumented chase car, to calibrate a polynomial model for acceleration. The polynomial model was formulated to satisfy the realistic condition, at the start and end of the departure movement, of zero 
acceleration $(a=0)$ and zero jerk $(d a / d t=0)$. The model assumes that the acceleration at any time, $t$, is computed using the following equations:

$$
\begin{aligned}
a_{t} & =b \theta\left(1-\theta^{m}\right)^{2} \quad(\mathrm{~m}>-0.5) \\
b & =\frac{2(m+1)(m+2)}{m^{2}} a_{\text {avg }} \\
m & =\frac{15-27 \rho+\sqrt{\left(81 \rho^{2}-138 \rho+73\right)}}{12 \rho-4} \\
\rho & =\frac{u_{\text {avg }}-u_{0}}{u_{f}-u_{0}} \\
\theta_{m} & =(1+2 m)^{-\frac{1}{m}}
\end{aligned}
$$

where

$$
\begin{array}{ll}
u_{f} & =\text { final speed of the vehicle } \\
u_{0} & =\text { initial speed of the vehicle } \\
u_{a v g} & =\text { average speed of the vehicle }\left(d_{f} / t_{f}\right) \\
b & =\text { vehicle-specific calibrated parameter } \\
a_{a v g} & =\text { average acceleration rate of the vehicle }\left(u_{f}-u_{0} / t_{f}\right) \\
\theta & =\text { relative time at instant }\left(t / t_{f}\right) \\
\theta_{m} & =\text { relative time at which vehicle attains maximum acceleration }\left(t_{m} / t_{f}\right) \\
t_{f} & =\text { time required for the vehicle to attain final speed } \\
t_{m} & =\text { time at which vehicle attains maximum acceleration, and } \\
d_{f} & =\text { final distance traveled by the vehicle }
\end{array}
$$


The speed and distance at any time, $t$, can be computed as per the following equations:

$$
\begin{aligned}
& u_{t}=u_{0}+t_{f} b \theta^{2}\left[0.5-\frac{2 \theta_{m}}{m+2}+\frac{\theta^{2 m}}{2 m+2}\right] \\
& d_{t}=u_{0} t+t_{f}^{2} b \theta\left[\frac{1}{6}-\frac{2 \theta^{m}}{(m+2)(m+3)}+\frac{\theta^{2 m}}{(2 m+2)(2 m+3)}\right]
\end{aligned}
$$

The initial and final conditions should be known to use the above polynomial model to compute the speed and acceleration at any intermediate time instance, $t$, which is not always available; and therefore, the linear model may be more practical to be used in different applications.

\subsection{Driving Simulator}

A key factor in the success of the proposed system is its inclusion of human factors of the driver of the equipped vehicle. This includes estimating the driver's perception-reaction time from the time a signal is triggered by the system to the time the vehicle starts to accelerate. The human factors also include the ability to predict the driver's selection for the desired vehicle acceleration rate, which is based on driver's characteristics and vehicle performance and can assume any value between a minimum acceleration rate and the maximum acceleration rate that can be performed by the vehicle. 
Regression models have been calibrated to precisely predict both the perceptionreaction time and the acceleration rate selected by different drivers. The driver simulator facility at the Department of Civil Engineering, Ryerson University has been utilized for the experimental work to collect the data used to calibrate the required models. The simulator system at Ryerson includes an experimental passenger car (shown in Figure 2.3) where the throttle, steering wheel, and brakes are all connected to driving simulator system to measure driver's response to different scenario events that are generated by a PC computer using a wall projector. The driving simulator uses the STISIM simulation system developed by Systems Technology Inc (STISIM 2009). The system uses setup and event files to run the simulation, allowing vehicle dynamics to be modified and driving scenarios to be created using scenario definition language (Allen et al 2004).

The scenario definition language (SDL) was developed to minimize the effort required to specify experimental designs. It frees the user from having to program visual data bases as is the case with many other CGI-based simulators. The simulation system consists of STISIM software and PC-compatible hardware components for producing visual scenes and auditory displays relevant to driving. It produces real-time driving scenarios that are projected on a screen in front of the subject driver (Figure 2.4).

Driving simulators have been broadly used for training, vehicle design, and safety research. They have also been used to assess different aspects of human factors, including pavement marking effect (Horberry et al. 2006), perception of traffic signs (Dutta et al. 2004), gap acceptance behaviour (Alexander et al. 2002), passing 
manoeuvre (Jenkins and Rilett, 2005), crash avoidance manoeuvre (Smith et al. 2002), driving distraction due to the use of mobile phones (Rakauskas et al. 2004), and human behaviour at high-risk situations (Yan et al. 2008). The use of driving simulators has many advantages over similar real-world or on-road driving research, including experimental control, efficiency, expense, safety, and ease of data collection (Nilsson, 1993). Driving simulators also have a potential to identify highway design problems and explain interaction between drivers and roadway surroundings (Yan et al. 2008).

To assess the validity of driving simulators of representing actual driving tasks, two primary areas should be validated (Jamson 1999); namely, physical and behavioural validation. Physical validity measures the degree to which the simulator dynamics and visual system reproduce the vehicle being simulated. As the world's most sophisticated driving simulator, the U.S. National Advanced Driving Simulator (NADS) needs a detailed, highly accurate vehicle dynamics simulation to predict the movements of the simulated vehicle in response to both control and disturbance inputs. Several types of vehicle dynamics models in NADS have been validated at the absolute validity level, including Ford Taurus, Jeep Cherokee, Chevrolet Malibu, and a tractor-semitrailer (Salaani and Heydinger, 2000).

Behavioural validation refers to a simulator's ability to induce the same response from a driver as would be performed in the same situation in real life (Jamson 1999). Blaauw (1982) proposed two types of driving behavioural validity, including:

- Absolute validity where the numerical values between the two systems are the same; and 
- Relative validity where differences found between experimental conditions are in the same direction, and have a similar or identical magnitude on both systems.

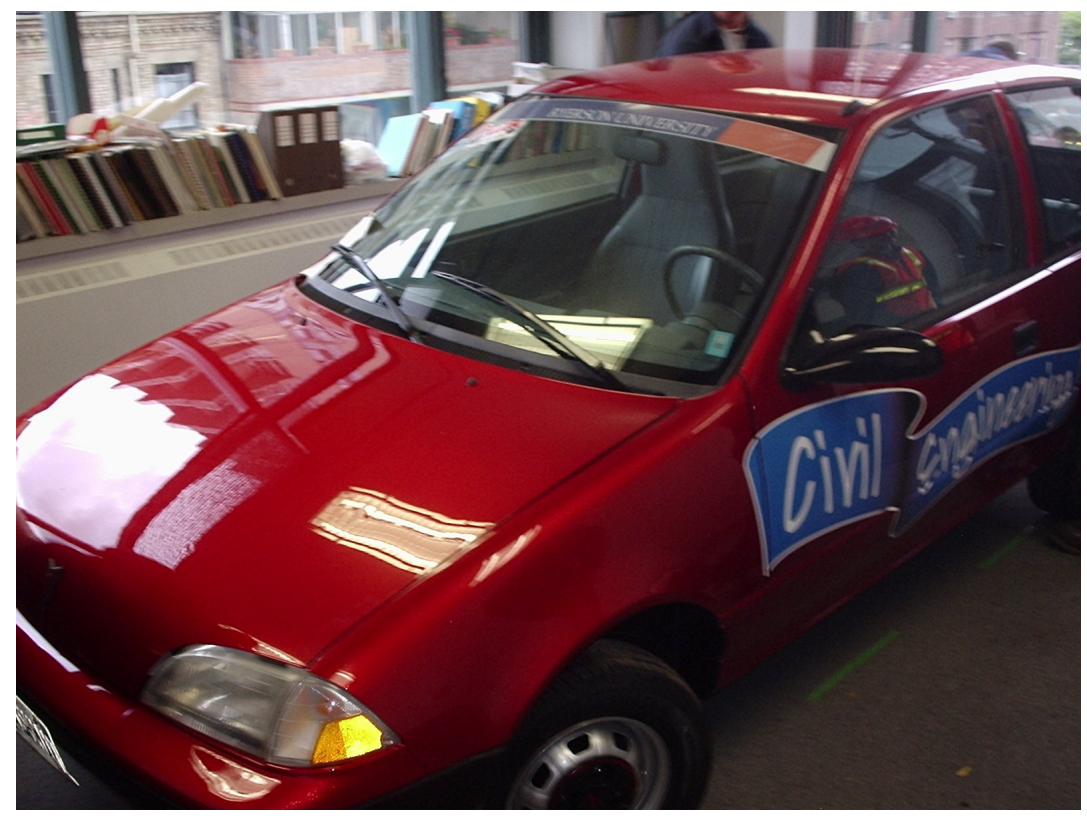

Figure 2.3 The experimental car used for simulation at Ryerson University

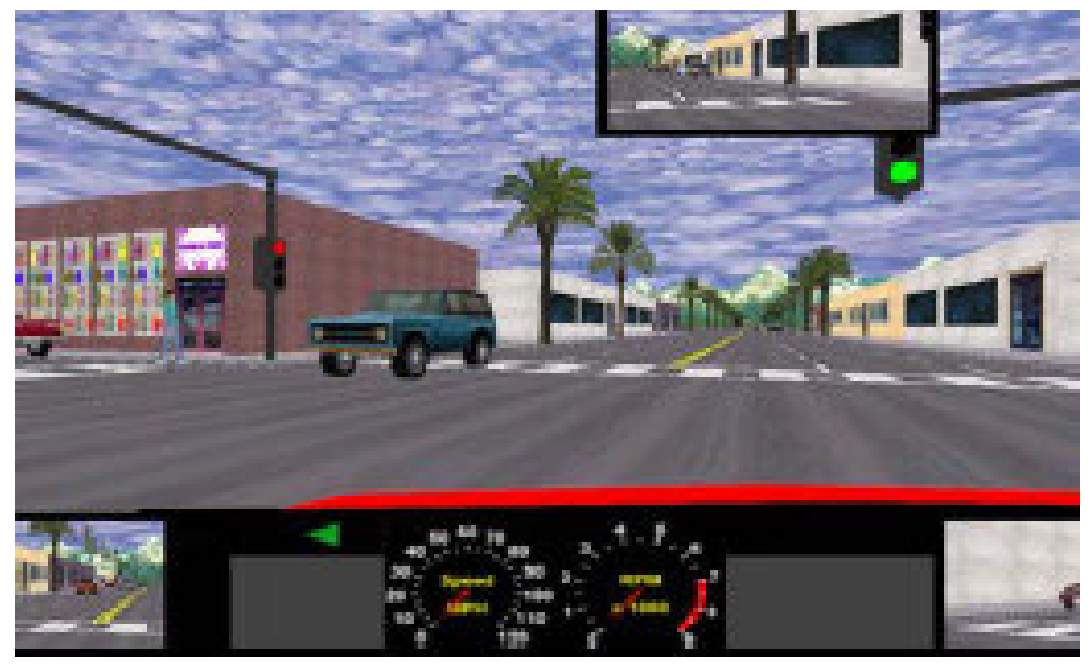

Figure 2.4 Screen shot from driving simulator by STISIM 
Many previous validation studies related to driving speed behaviours have been conducted to evaluate if drivers have similar speed performances in driving simulators as those measured in the real-world or a real instrumented car. At the University of Central Florida, a study was conducted to evaluate if the fix-base driving simulator could provide a realistic driving experience (Klee et al., 1999). The results indicated that the drivers behaved similarly at 10 of 16 designated locations along the road, but the difference in mean speeds between the simulator and the field indicated a tendency of drivers to travel at slower speeds in the simulator.

A speed validation study was conducted (Godley et al. 2002) that compared speed measurements of an instrumented car and the driving simulator in two separate experiments where the tested roadways contained transverse rumble strips at three sites, as well as three equivalent control sites without rumble strips. It was found that participants reacted to the rumble strips, in relation to their deceleration pattern on the control road, in very similar ways in both the instrumented car and simulator experiments, establishing the relative validities. However, the results failed in absolute validity because participants generally drove faster in the instrumented car than the simulator.

Another validation study for speed and lateral position evaluated driving behaviour in a simulated road tunnel (Tornros 1998) where similar conclusions were drawn that behavioural validity in absolute terms was not quite satisfactory, especially regarding the choice of speed, whereas relative validity was achieved for both speed and lateral position. 
It was found that many realistic results can be obtained by driving simulators, including absolute validity of route choice behaviour, relative validity of speed and lateral control behaviour; and the presence of a moving base and possibly a higher image resolution might increase the validity of a driving simulator (Kaptein et al. 1996). In the Interuniversity Research Center for Road Safety (CRISS), a simulator speed validation study focused on the effectiveness of temporary traffic signs on highways (Bella 2005). Speed measurements were conducted for both field observations and driving simulator experiments in the transition area, the activity area, the termination area, and the advance warning area. The results showed that differences between the speeds observed in the real situation and those measured with the simulator were not statistically significant and therefore validated the driving simulator in absolute terms.

A further simulator validation study for highway deceleration lane design (Bella et al. 2007) indicated that the speeds in driving simulator were also similar to the field data, but standard deviation in simulation is higher than in reality. Another recent research was conducted to ascertain the validity of a driving simulator in determining the effectiveness of temporary traffic control devices in a work zone during night time hours. Spot speeds were observed in both field and driving simulator at three locations in a freeway work zone (McAvoy et al. 2007). However, it was found that the statistical tests indicated that a simulator study may not reproduce the mean travel speed of a field study for night time driving conditions through a work zone in either absolute or relative validity terms. 
Another study was conducted to validate the driving behaviour of older adult drivers (60-90 years) in a PC-based simulator (Lee 2002) where an overall measure index have been established to evaluate drivers' performances, including driving speed, confidence on high speed, traffic rule compliance, decision and judgment, road use obligation, working memory, and other measures. Based on that study, it was found that the use of driving simulator may be a safer and more economical method than the on-road testing to assess the driving performance of drivers.

\subsection{Vehicle Tracking}

Vehicle tracking in collision warning systems is usually performed by using Kalman filter (Kalman 1960; Grewal and Andrews 1993; Maybeck 1979). The Kalman filter performs tracking by estimating the state of dynamic objects (i.e., the approaching vehicles) at different time intervals from a series of incomplete and noisy measurements (taken by detection sensor) and provides accurate continuouslyupdated information about the position and speed of those approaching vehicles. A bounding box is placed around the predicted positions of approaching vehicles and logic is used to determine if the detection is within that bounding box and hence is associated with a specific track. The trajectory of a detected vehicle is estimated using the following state vector:

$$
\hat{x}_{k}=\left(\rho_{k}, \phi_{k}, d_{k}, v_{k}, a_{k}\right)
$$


where $\rho_{k}, \phi_{k}$ are the polar coordinates of the centre of the bounding box, $\left(d_{k}, v_{k}, a_{k}\right)$ relate to the dynamic state, and $\mathrm{k}$ is the time series. The vehicle position is then calculated by:

$$
z_{k}=H \hat{x}_{k-1}+w_{k}
$$

where $\hat{x}_{k-1}$ is the prior estimation of state vector, $w_{k}$ is the measurement error, and $H$ relates to the measurement matrix to the centre of the detected vehicle. The following are different cases for associating tracked vehicles with boundary boxes:

- If the detected vehicle does not fall within the boundary box of an existing track or candidate track, that vehicle is marked as a candidate track and a new boundary box is created for it.

- If the detected vehicle is found to be associated with a specific boundary box for a candidate track, it is promoted to be a track.

- If a track is not associated with any detection for some specified number of updates it is maintained, or "coasted", on its current trajectory for a specified number of updates after which it is deleted. In the coast mode the last update of state variables is retained. The track remains ready for an association.

- If a candidate track is not associated with any new detection, it is deleted. 


\section{CHAPTER 3: ALGORITHM DESIGN FOR LEFT-TURNING VEHICLES}

This chapter describes the architecture of the proposed algorithm used to warn against imminent left-turn collisions and the method used to estimate the time required for any detected vehicle to reach the intersection and also the method used to estimate the time required for the turning vehicle to complete the left turn movement and how to compare the two values to ensure that the driver of the host vehicle can safely complete the left-turn movement without being involved in a collision.

\subsection{Algorithm Architecture}

The proposed algorithm utilizes a detection sensor that is installed at the front-left corner of the turning (target) vehicle, as shown in Figure 3.1. The detection sensor can be either a radar sensor similar to the ICA and ICAV systems, or a laser scanner similar to the INTERSAFE system. The detection sensor is installed at the left-side front corner of the host (target) vehicle to detect approaching (bullet) vehicles in the opposite-direction traffic stream. Using the detection sensor, along with a processing unit and a driver-vehicle interface (DVI) unit, the algorithm follows the procedures shown in Figure 3.2 to determine whether a 'safe' message should be displayed to the driver of the turning (target) vehicle. 


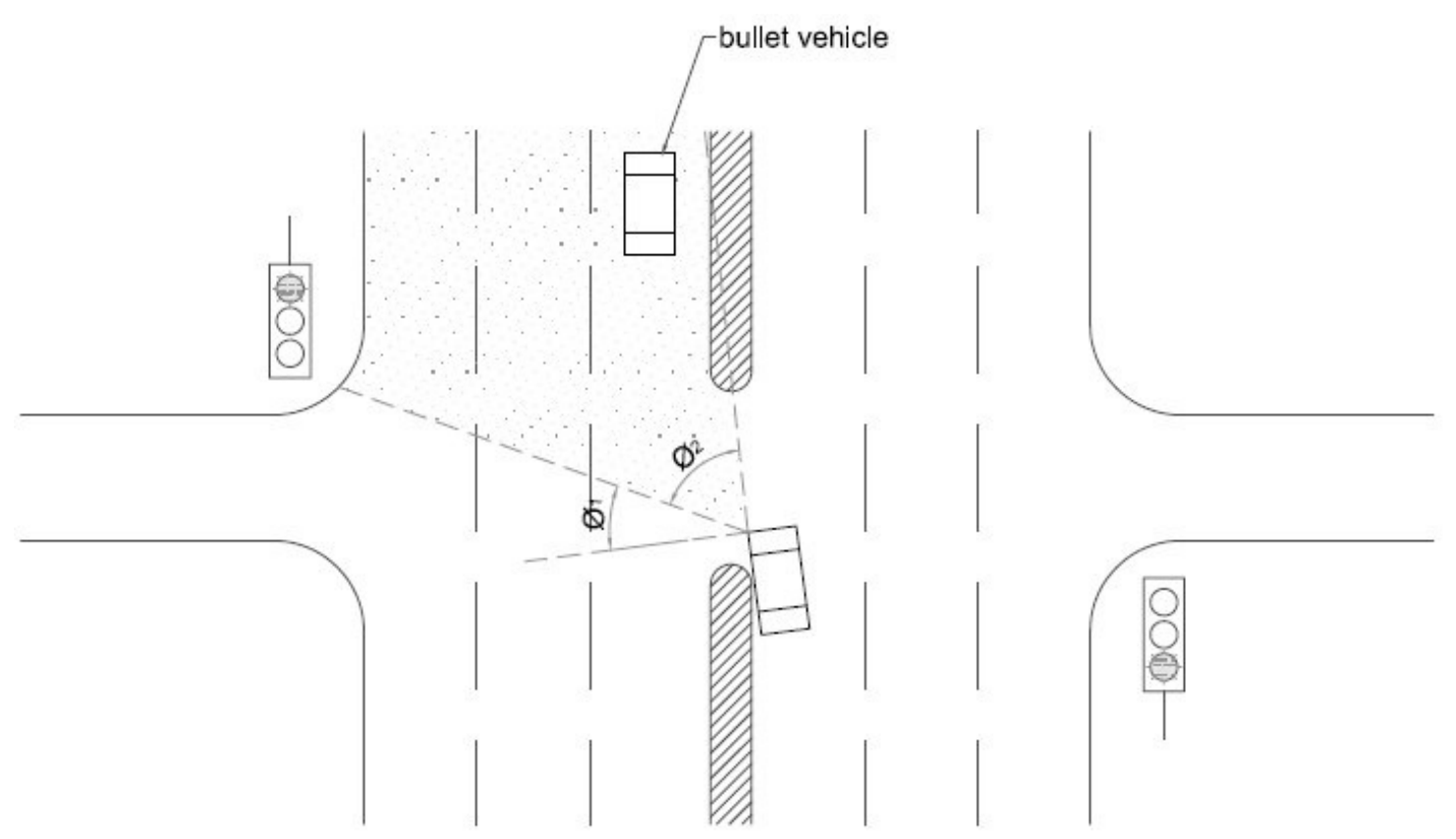

Figure 3.1 Configuration of the proposed detection sensors

The procedures followed by the algorithm are as follows (Dabbour and Easa 2009):

1. Once the brakes are activated and the detection sensor detects moving objects within its coverage area (the opposite traffic lanes) the system displays a warning message to the driver by default. That message could be visual, auditory, haptic, or a combination of two methods. The message is not deactivated until the algorithm confirms that a safe left turn is available as per the subsequent steps;

2. The system estimates the time required for each detected vehicle, $t_{\text {bullet, }}$ to reach the intersection using the methodology presented later in this chapter; 
3. The system also estimates the time required for the turning vehicle, $t_{\text {target, }}$ to complete the left turn movement using the methodology presented later in this chapter;

4. If the time required for the opposite vehicle to reach the intersection, $t_{\text {bullet, }}$ was found to be greater than the time required to complete the left turn, $t_{\text {target, }}$, by more than the factor of safety used, a 'safe' message will be displayed to the driver of the turning vehicle to allow him/her to start the left turn movement; or

5. If the time required for the opposite vehicle to reach the intersection, $t_{\text {bullet, }}$ was found to be greater than the time required to complete the left turn, $t_{\text {target}}$, by less than the factor of safety used, the warning 'not safe' message will continue to be active and the system will repeat the above algorithm until the criterion shown in the previous step is met (i.e., a proper gap is found).

It should be noted that the algorithm is capable of recognizing different approaching vehicles on different lanes and the above procedures are followed for each vehicle on each lane and the 'safe' signal is not activated until all lanes are clear of approaching vehicles that could collide with the turning vehicle, as will be discussed later in this chapter.

\subsection{Specifications of Detection Sensor}

The detection sensor sends a beam every time interval, $t$, to scan the traffic lanes in the opposite direction of the road. The time interval ranges from $0.04 \mathrm{sec}$ to 1.5 
sec, depending on the type of the detector used. From earlier research (NHTSA 2004) an update rate of $10 \mathrm{~Hz}$, with 0.1 second time interval is recommended to provide acceptable range and range-rate resolution.

The width of the beam is designed so that the angle between the outer left edge of the beam and the face plane of the vehicle, $\Phi_{1}$, is within the range $\left(10^{\circ}-22^{\circ}\right)$, as shown in Fig. 3.1. This is to ensure no opposite vehicle, which may potentially collide with the turning vehicle, is outside the coverage area of the radar beam. The maximum azimuth angle, $\Phi_{2}$, is within the range $\left(55^{\circ}-70^{\circ}\right)$ to cover three lanes in the opposite direction in addition to a median.

Based on the findings of earlier research (NHTSA 2000; US DOT 2003; NHTSA 2004) the other required specifications for the detection sensor used are as follows:

1. Maximum range no less than $150 \mathrm{~m}$ to detect all vehicles traveling in the opposite direction that may collide with the host vehicle. This value is calculated by assuming $85^{\text {th }}$ percentile maximum running speed on the major road of approximately $96.6 \mathrm{~km} / \mathrm{h}$ (Fitzpatrick et al. 2003), which allows approximately 5.5 seconds for departure.

2. Opening (azimuth) angle (horizontal) $55^{\circ}-70^{\circ}$ (as explained above).

3. Opening (azimuth) angle (vertical) $4^{\circ}-8^{\circ}$.

4. Resolution of azimuth angle $0.1^{\circ}$ or better.

5. Maximum data latency 0.05 seconds to minimize the processing time and ensure realistic performance of the system without causing nuisance to the driver of the equipped vehicle. 
6. Maximum range-rate no less than $30 \mathrm{~m} / \mathrm{sec}$ (assuming maximum speed on the opposite direction of the major road of $108 \mathrm{~km} / \mathrm{h}$ ).

7. Range resolution is $0.05 \mathrm{~m}$ or better (given the small cycle time).

8. Update rate is $10 \mathrm{~Hz}$, with 0.1 -sec time interval.

The above are technology-independent minimum specifications and further research will be required to select the product that meets them. Possible candidates may include the following detection sensors:

1. The EVT-200 radar sensor produced by Eaton VORAD Technologies (VORAD 2009). That sensor was utilized by the ICA system (Jocoy et al. 1997; Pierowicz et al. 2000); however, the maximum opening angle provided by that sensor is smaller than required and technical modifications may be required to increase the opening angle.

2. The UMRR-09xx radar sensor produced by Smart Microwave Sensors (SMS 2009). That sensor may meet the minimum specifications required; however, given that it is fully customized, its price may be too high to be implemented for commercial use.

3. The LUX laser scanner produced by ibeo Automobile Sensor (IBEO 2009). That sensor emerged from the original ALASCA XT scanner that was utilized by the INTERSAFE system (Fuerstenberg and Chen 2007); however, further research may be required to ensure its functionality under different weather conditions. 


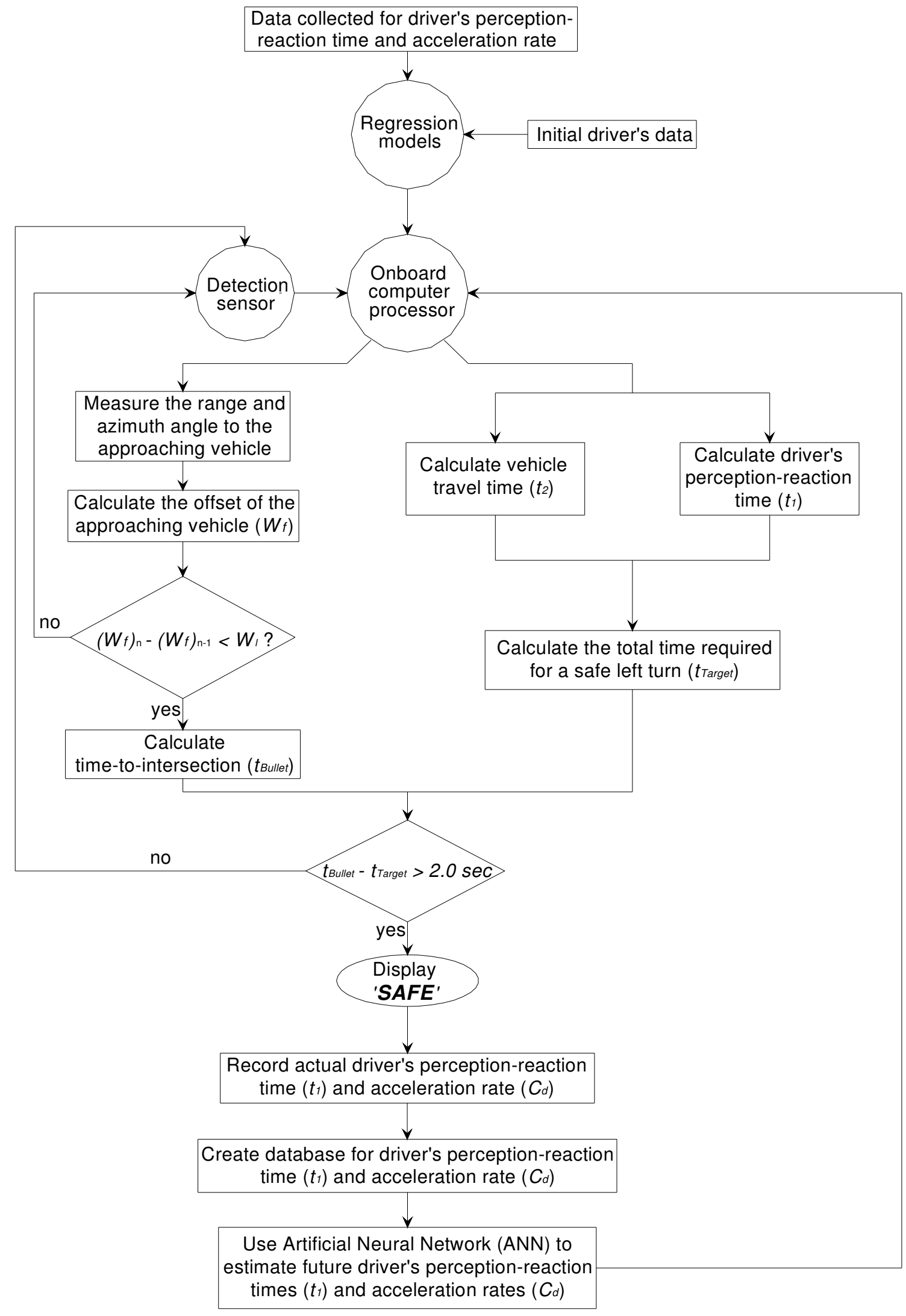

Figure 3.2 Flow chart for the left-turn algorithm 


\subsection{Effect of Lane Configuration}

The algorithm is designed to work with any number of opposite through lanes and it does not detect any vehicles outside the through lanes, as shown in Figure 3.3 and as will be discussed later in this chapter. It should be noted that the beam angle, $\Phi_{2}$, is typically designed for a maximum three through lanes in each direction as this is the maximum number in practice for through lanes in typical North American arterials (AASHTO 2001; TAC 1999). The detection sensor can also detect any right-turning vehicles, either in dedicated or shared lanes, and include them in the algorithm, regardless of the number of the through lanes, as shown in Figure 3.4.

In case if dedicated left-turn lanes exist, in addition to through lanes, the dedicated left-turn lane in the opposite direction is not be covered by the radar beam, as shown in Figure 3.5. This is due to the offsetting of left-turn lanes that is in practice and as was suggested by many researchers (Easa et al, 2005; Easa and Ali, 2005). However, even if the radar beam detects left-turning opposite vehicles, the algorithm can recognize that vehicles, in that opposite left-turn lane, are decelerating to nearly fully stop; and therefore will not include those vehicles in the calculations of the algorithm, as will be discussed later in this chapter.

It should be noted that the main objective of the proposed system is to compensate for the human error in perceiving the speed and acceleration of approaching vehicles that are visible fir the driver of the equipped vehicle, which means that it should not be considered as an aid for drivers to detect vehicles that are not visible to them. 


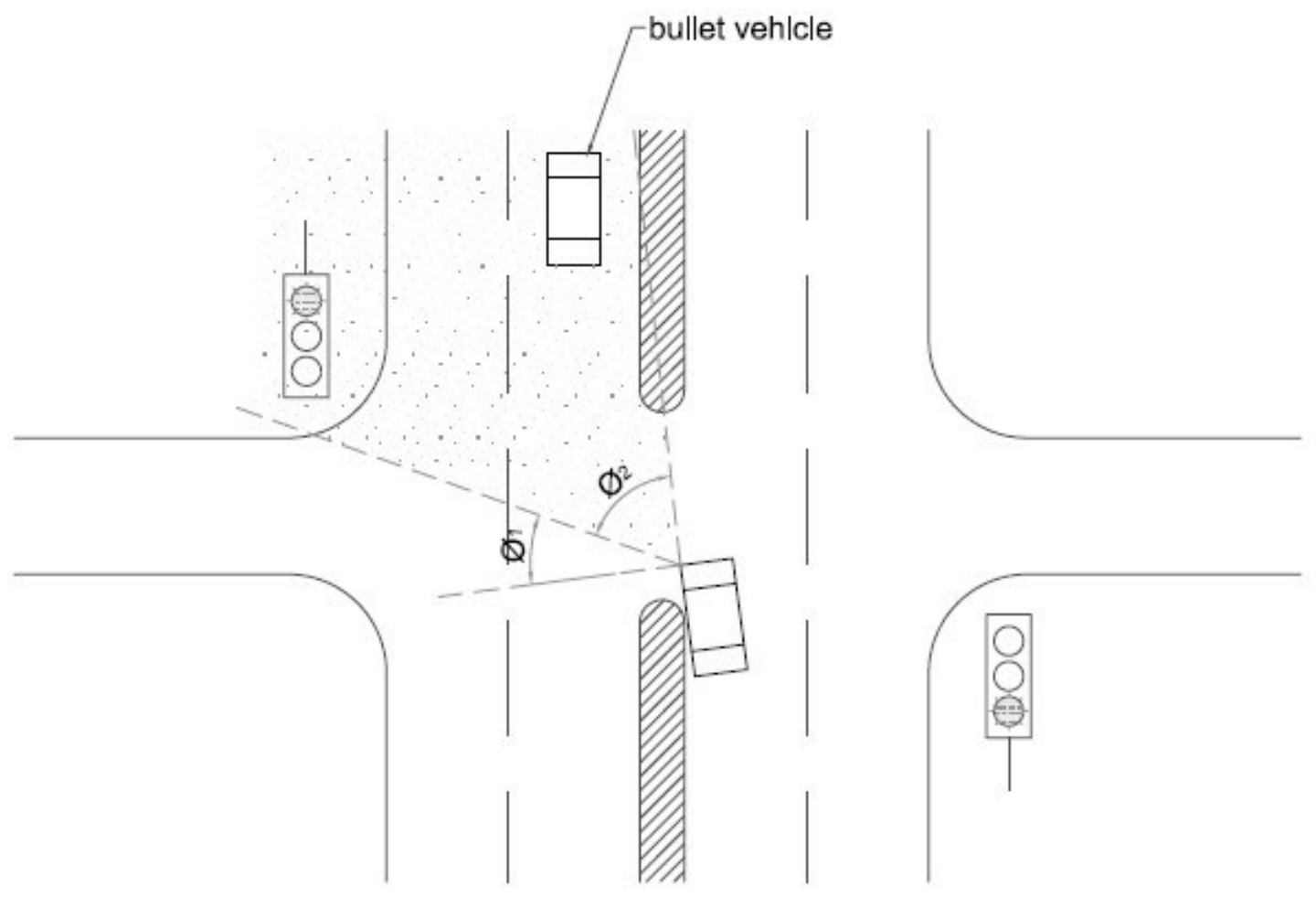

Figure 3.3 A typical roadway with less than 3 through lanes

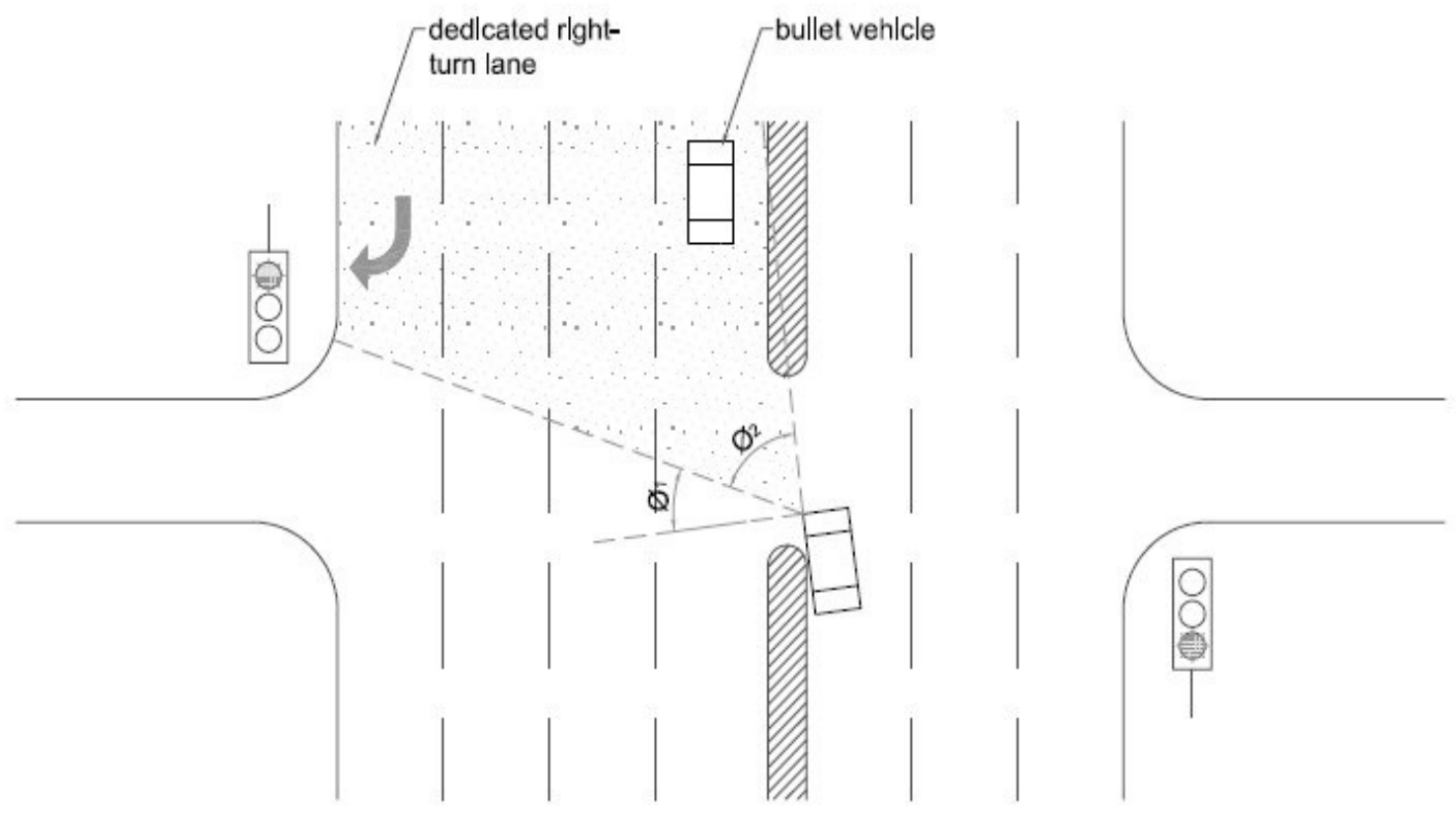

Figure 3.4 A typical roadway with dedicated right-turn lane 


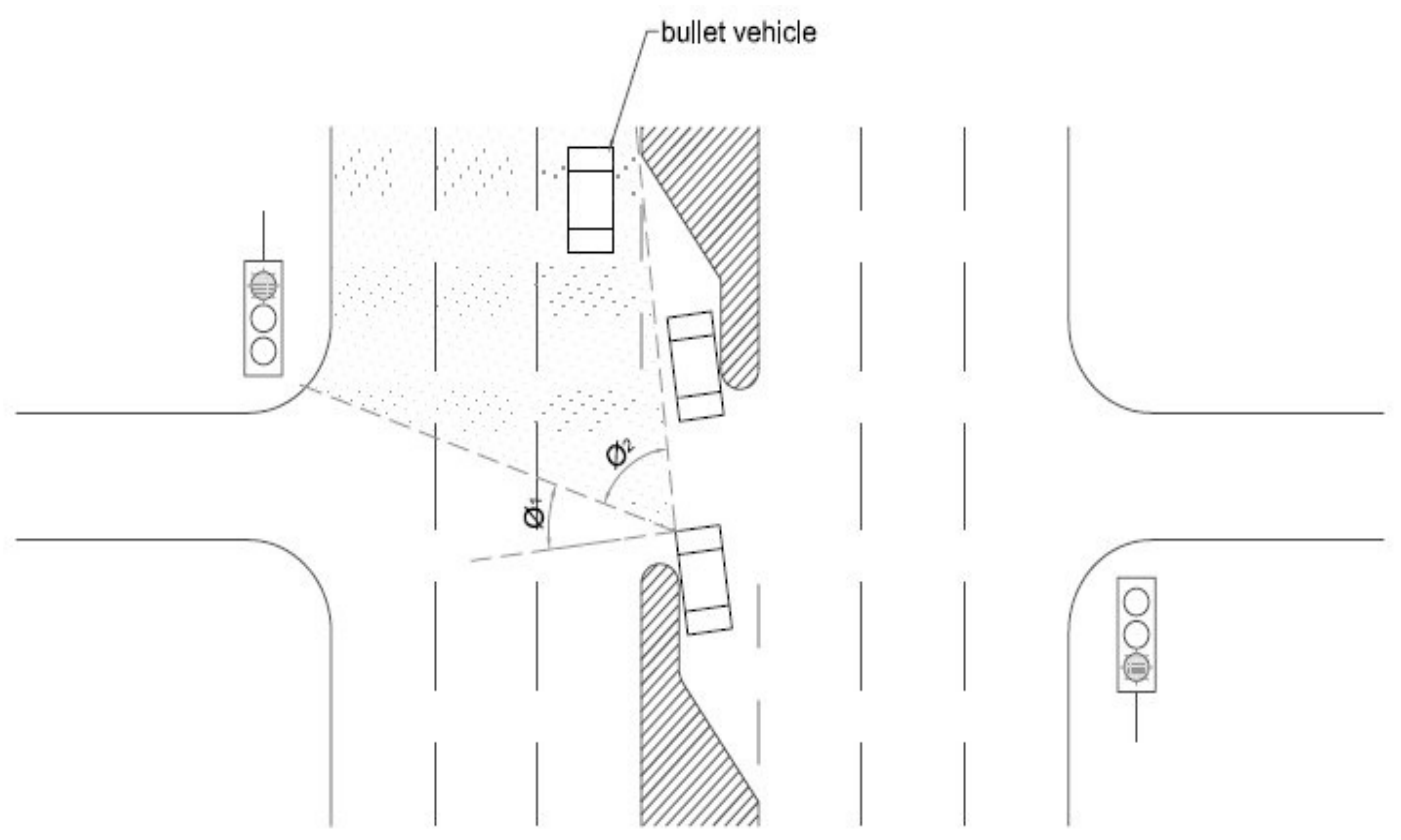

Figure 3.5 A typical roadway with dedicated left-turn lanes

\subsection{Calculating $t_{\text {bullet }}$}

A schematic illustration is shown in Figure 3.6 to depict the methodology for the algorithm that calculates the time required for the bullet vehicle to reach the intersection, $t_{\text {bullet, }}$ which can be summarised as follows:

1. A detection beam is generated at time $T$ to scan all opposite lanes. If no object is detected a 'safe' signal is displayed to the driver. Otherwise, the nearest object detected, vehicle $A$, is recorded at distance $d_{1}$ and azimuth angle $\theta_{1}$ (measured from the face plane of the detection sensor) where polar coordinates are used with the origin point to coincide with the location of the 
detection sensor, as shown in Figure 3.7. It should be noted that if multiple vehicles are detected, the algorithm is followed for each detected vehicle individually and a Kalman filter is used to track different vehicles as was explained in Chapter 2.

2. Another detection beam is generated at time $T+t$, where $t$ is the time interval for the detection sensor used, and the new location of vehicle $A$ is recorded at distance $d_{2}$ and azimuth angle $\theta_{2}$ where the same above polar coordinates are used, as shown in Figure 3.8.

3. If $d_{1}$ and $d_{2}$ were found to be equal, the algorithm will conclude that the object is not moving (e.g., a tree or a building in case of less than three through lanes). A 'safe' signal is displayed to the driver (unless another object is detected). It should be noted that generating a 'safe' signal is based on the assumption that the intersection is adequately designed for intersection sight distance as given by TAC 1999.

4. If $d_{2}$ was found to be greater than $d_{1}$, the algorithm will conclude that the object is moving in the same direction as for the turning (target) vehicle. This may occur in case the target vehicle is positioned in the same direction of the traffic so that it might detect another vehicle ahead of it, as shown in Figure 3.9. A 'safe' signal is also displayed to the driver (unless another object is detected).

5. If $d_{2}$ was found to be less than $d_{1}$, the distance traversed by the vehicle during the first time interval, $d_{v 1}$, is calculated as shown in Figure 3.10 using the following equation: 


$$
d_{v 1}=\sqrt{d_{1}^{2}+d_{2}^{2}-2 d_{1} d_{2} \cos \left(\theta_{1}-\theta_{2}\right)}
$$

6. A third detection beam is generated at time $T+2 t$ and the distance, $d_{3}$, and azimuth angle, $\theta_{3}$, for vehicle 'A' are recorded as shown in Figure 3.11.

7. The distance traversed by the vehicle during the second time interval, $d_{v 2}$, is calculated similar to calculating $d_{v 1}$ by using the following formula:

$$
d_{v 2}=\sqrt{d_{2}^{2}+d_{3}^{2}-2 d_{2} d_{3} \cos \left(\theta_{2}-\theta_{3}\right)}
$$

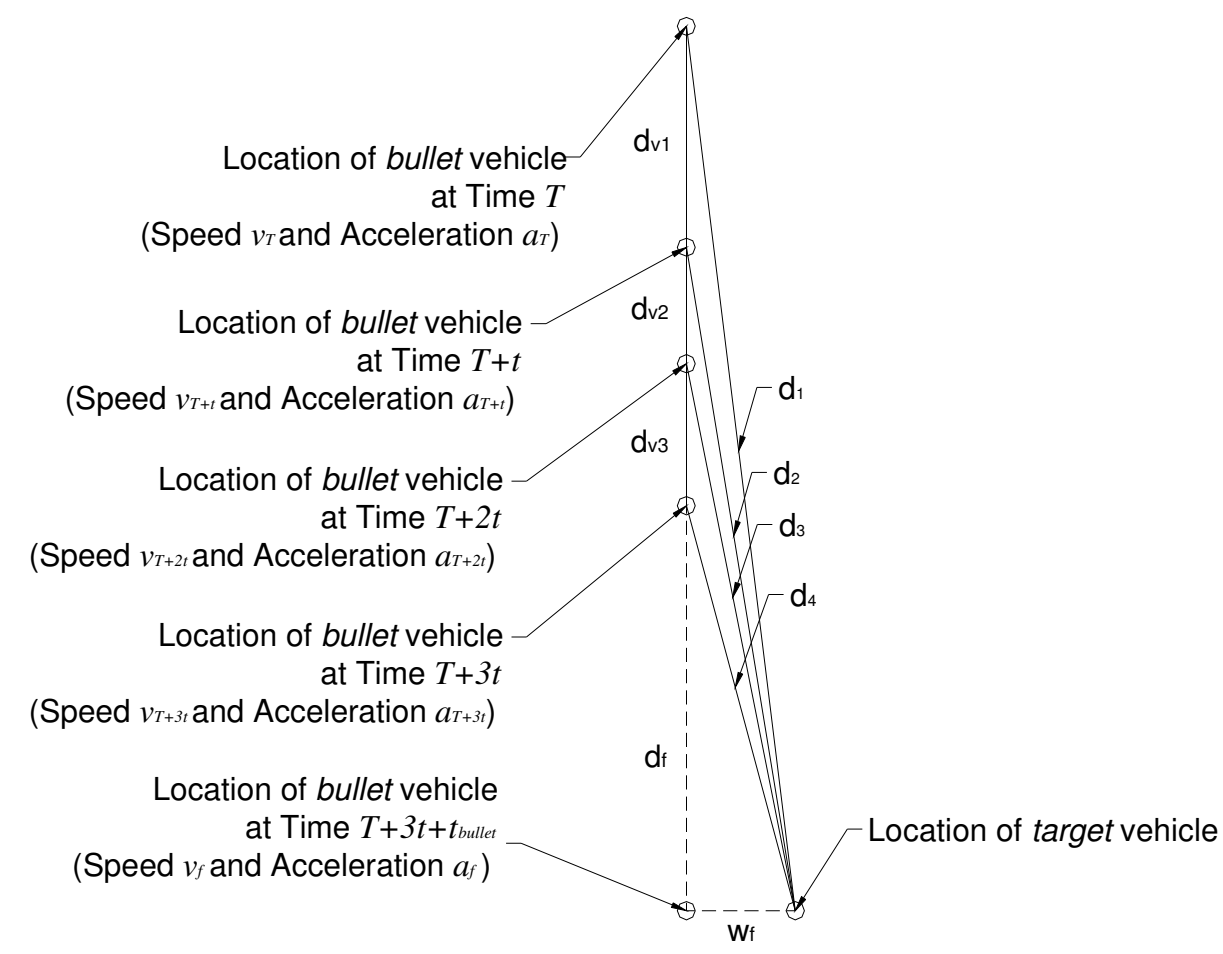

Figure 3.6 Schematic illustration of bullet vehicle locations at different time instances 


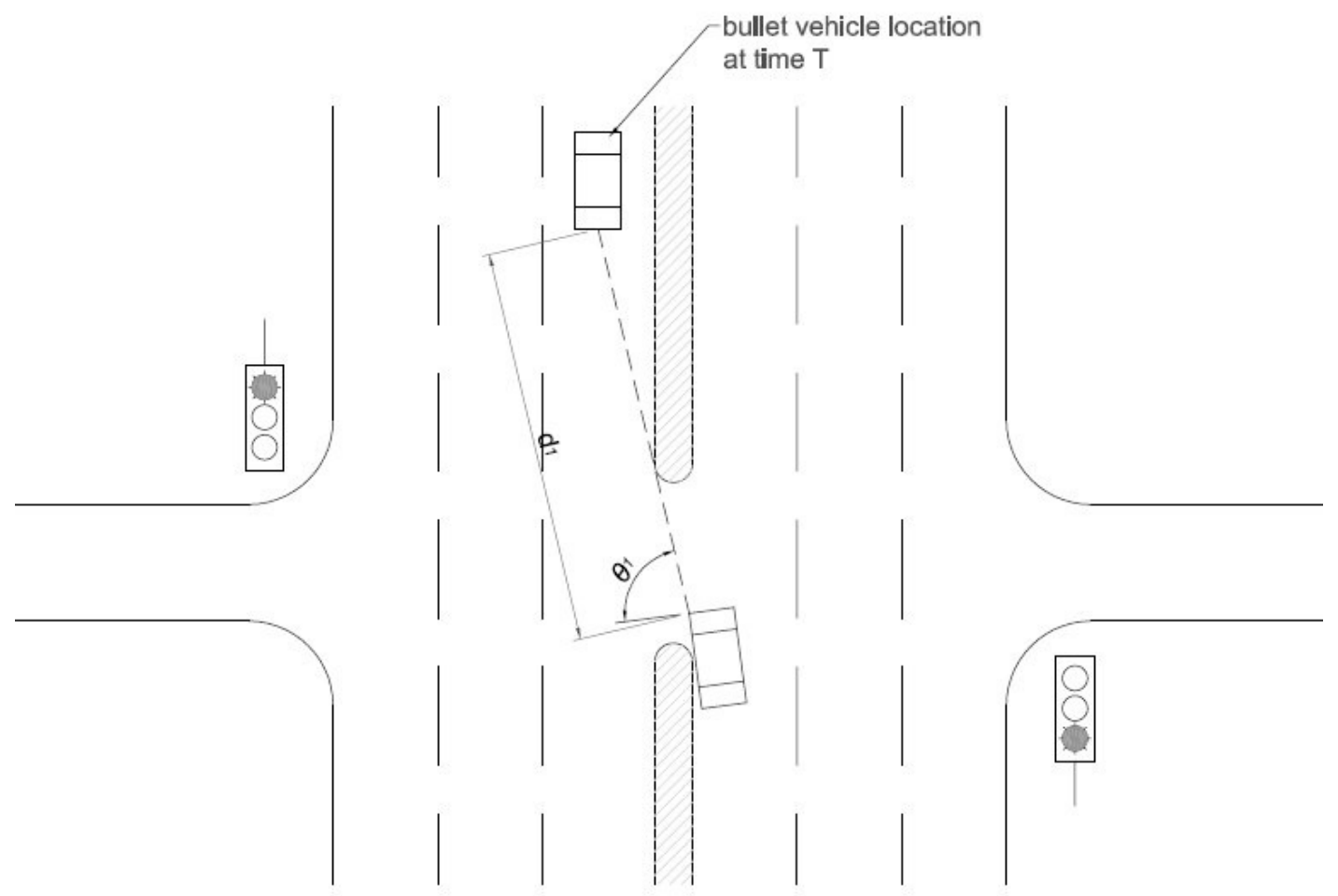

Figure 3.7 Calculating distance and angle at time $T$

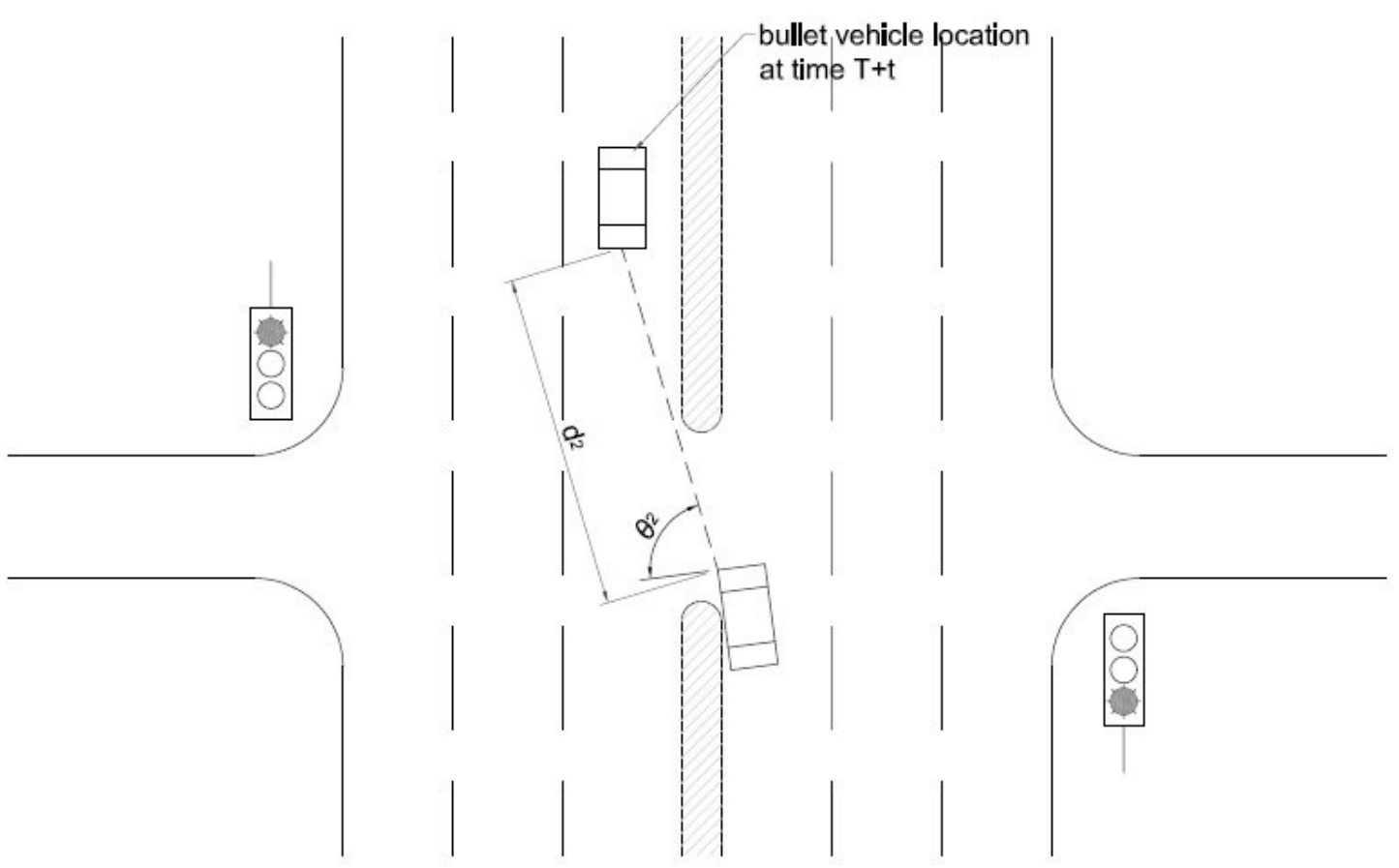

Figure 3.8 Calculating distance and angle at time $T+t$ 


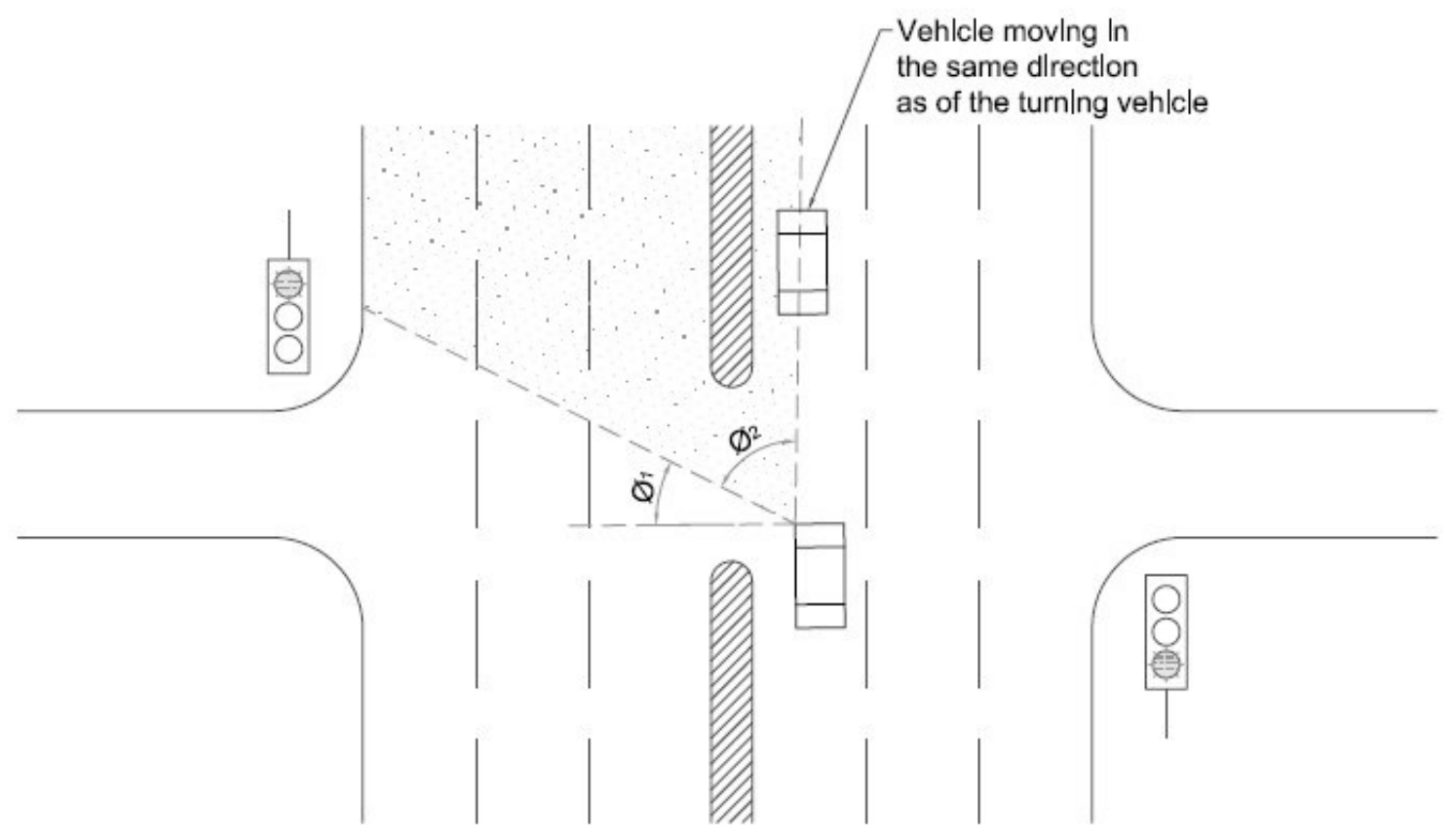

Figure 3.9 The case where the radar beam detects a vehicle in the same direction

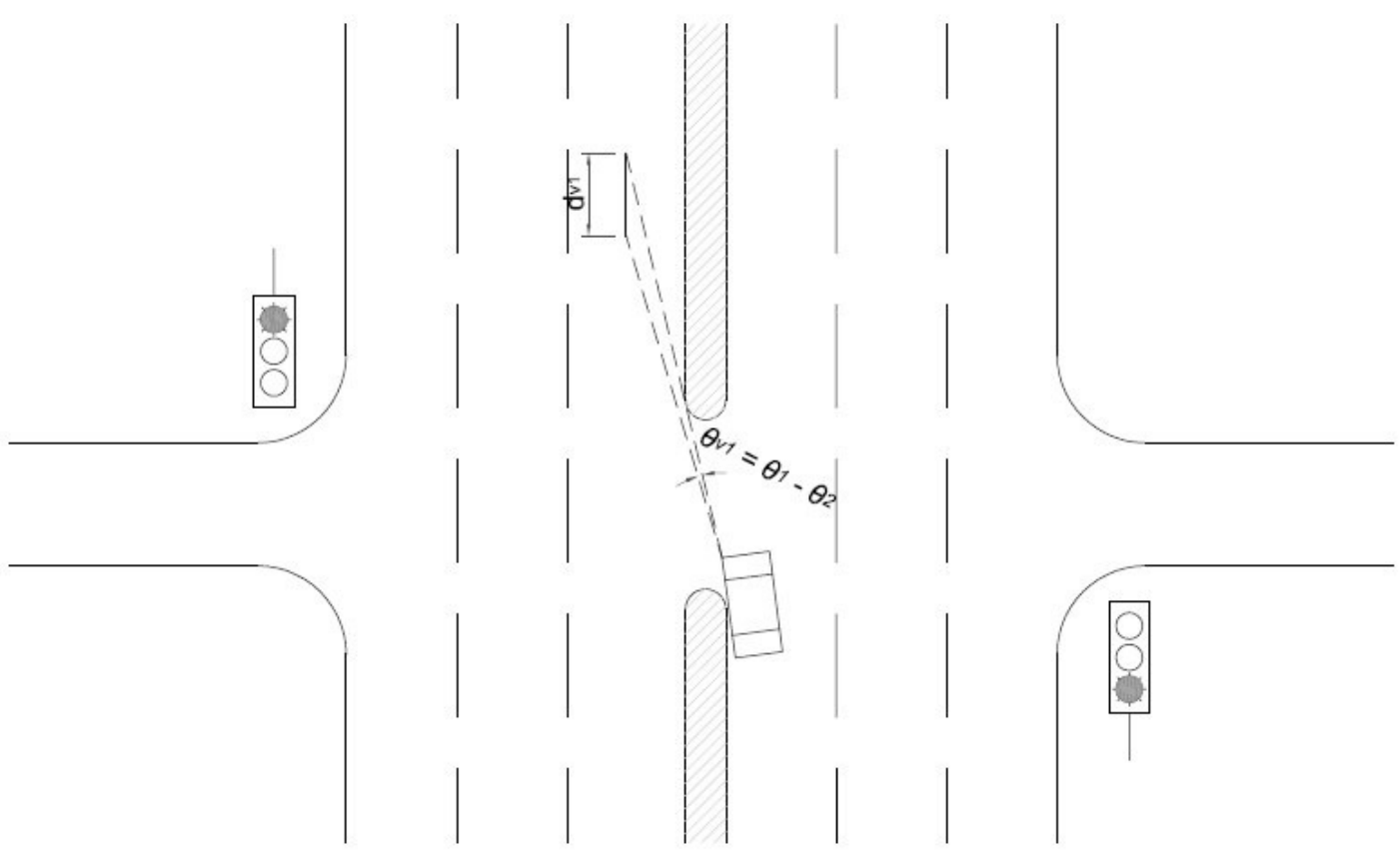

Figure 3.10 Calculating the distance traversed by the opposing vehicle 
8. Similarly, a fourth detection beam is generated at time $T+3 t$. The distance, $d_{4}$, and azimuth angle, $\theta_{4}$, are both measured, and the distance traversed by the vehicle during the third time interval, $d_{v 3}$, is also calculated by using the following formula as shown in Figure 3.12:

$$
d_{v 3}=\sqrt{d_{3}^{2}+d_{4}^{2}-2 d_{3} d_{4} \cos \left(\theta_{3}-\theta_{4}\right)}
$$

9. Given the small time intervals used, the time-acceleration relationship can be assumed as a linear model with slope $r$, which is the rate of change in acceleration as represented by the following equation:

$$
\frac{d a}{d t}=r
$$

It should be noted that the linear decay relationship, as was discussed in Chapter 2, cannot be applied for the approaching vehicles as the parameters $\alpha$ and $\beta$ that represent the mechanical characteristics of the approaching vehicle should be known where the parameter $\alpha$ represents the maximum acceleration that can be provided by the engine at the start of the movement, and the parameter $\beta$ represents the rate of decrease in acceleration as the speed increases. In reality, those parameters cannot be known for all approaching vehicles; and therefore, the linear decay 
acceleration function cannot be applied in this case. Based on that, the acceleration rate of an approaching bullet vehicle was assumed to have a linear relationship with the time. From the actual acceleration profile shown in Figure 3.13 (Wang et al. 2004) and given the small time period for the analysis, the error resulted from assuming a linear relationship is expected to be minimal.

10. The acceleration, speed and distance at any time, $t$, can be computed using the following equations (where $a_{T}, v_{T}$ and $d_{T}$ are the initial acceleration, speed and distance, respectively):

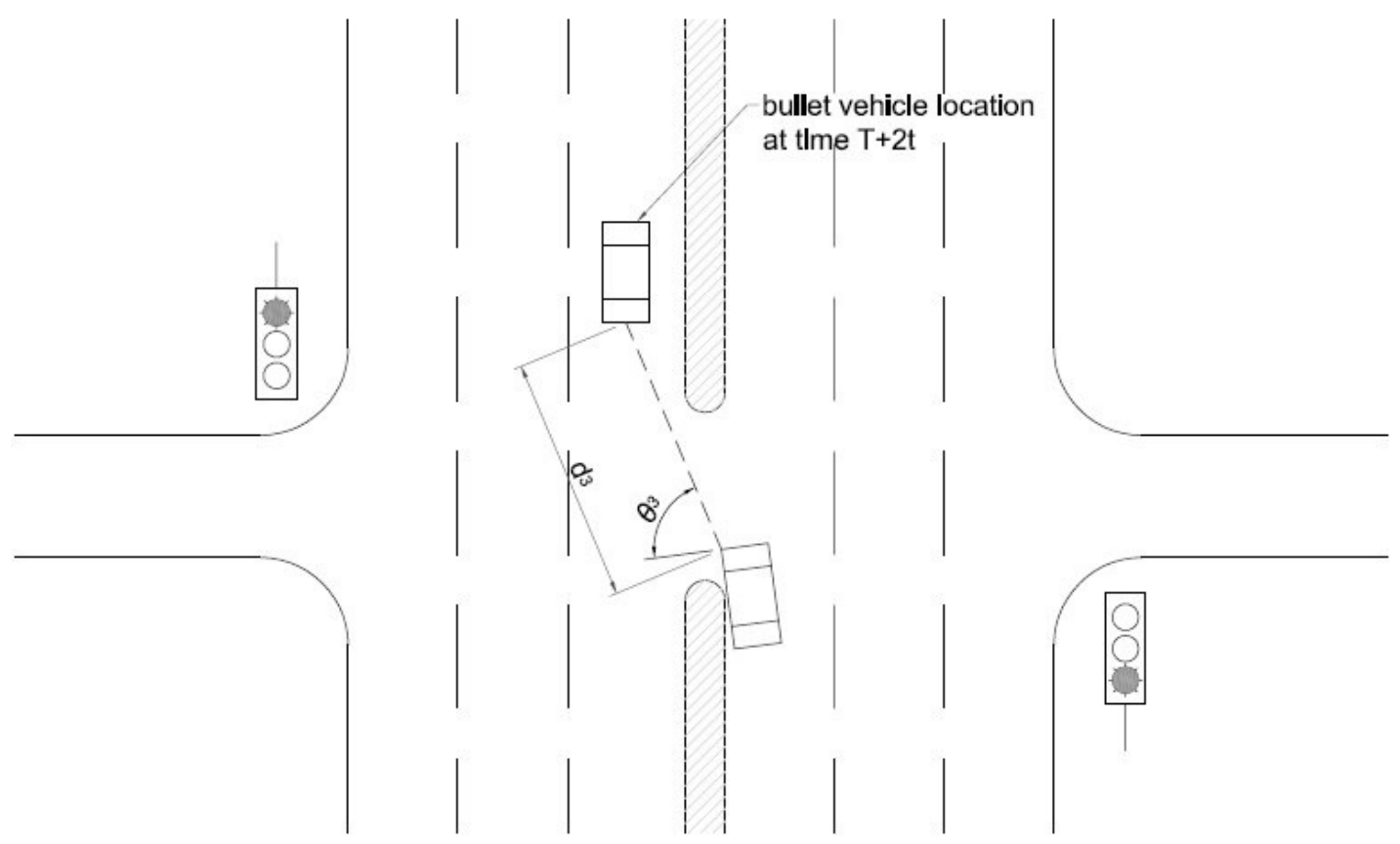

Figure 3.11 Calculating the distance at time $T+2 t$ 


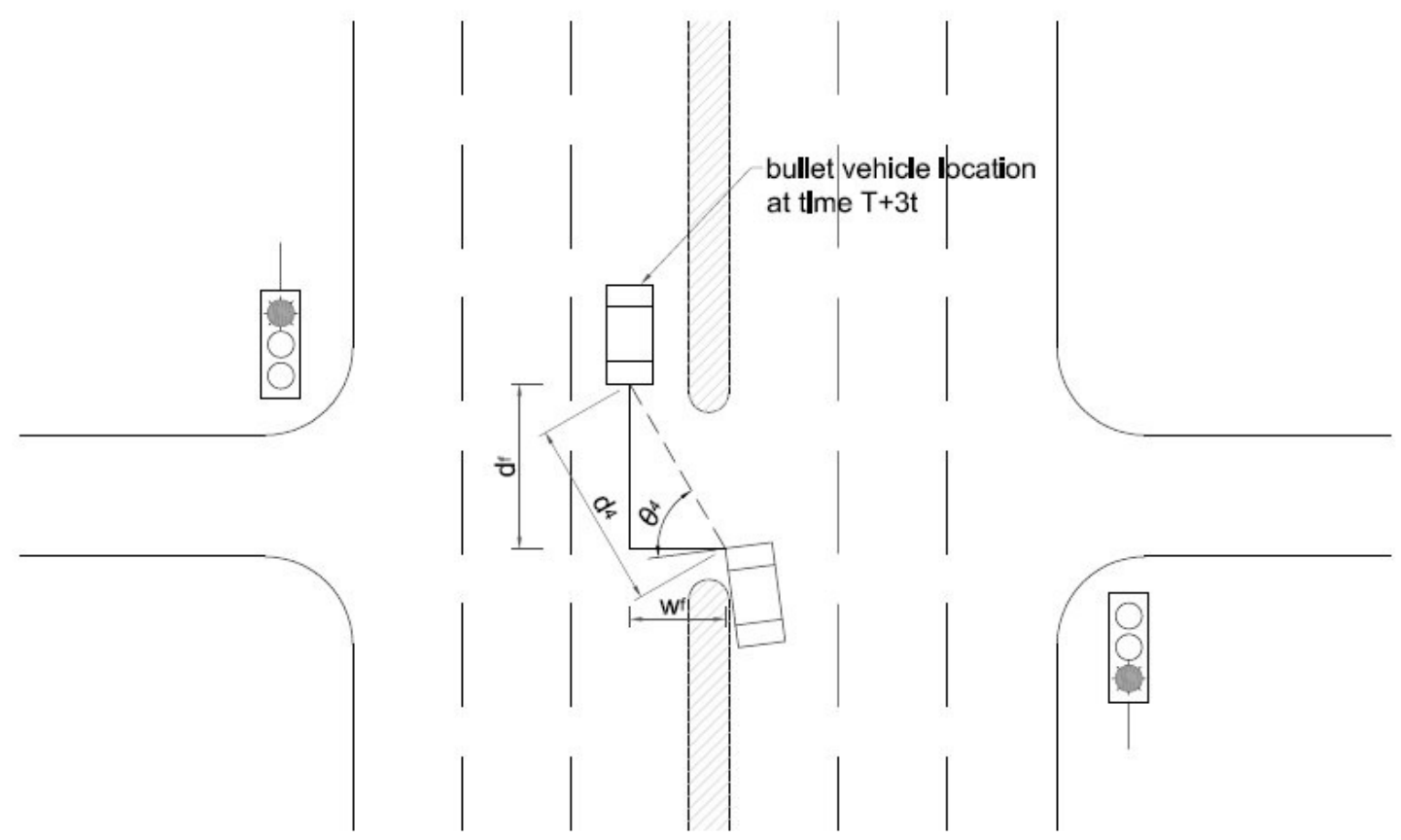

Figure 3.12 Calculating the distance at time $T+3 t$

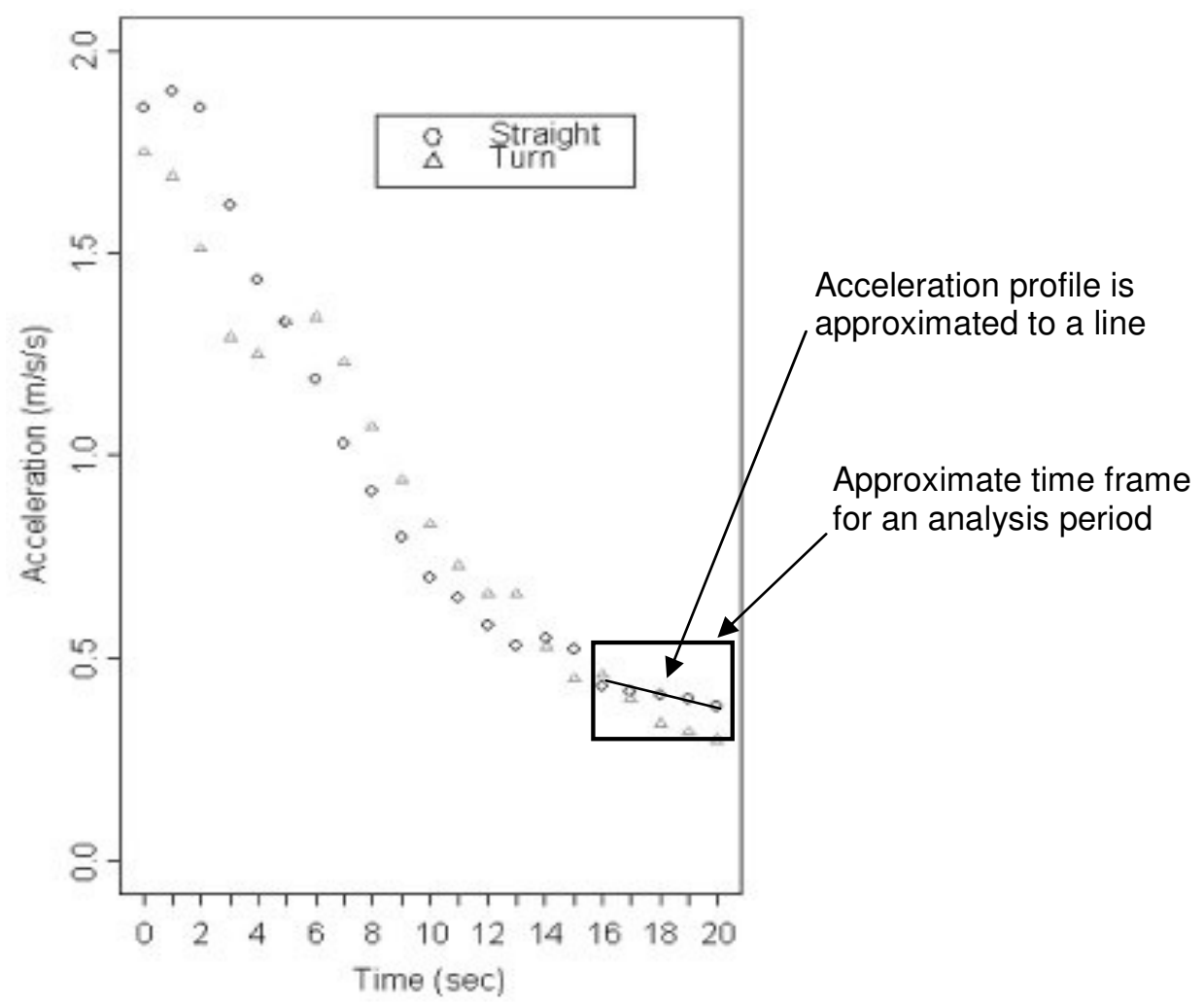

Figure 3.13 Observed acceleration profile [Wang et al. 2004] 


$$
\begin{aligned}
& a_{t}=\int_{0}^{t} \frac{d a}{d t} d t=a_{T}+r t \\
& v_{t}=\int_{0}^{t} a_{t} d t=v_{T}+a_{T} t+\frac{1}{2} r t^{2} \\
& d_{t}=\int_{0}^{t} v_{t} d t=d_{T}+v_{0} t+\frac{1}{2} a_{T} t^{2}+\frac{1}{6} r t^{3}
\end{aligned}
$$

11. By applying the above three equations at different time intervals, the acceleration, speed and distance at time $T+t$ are computed as per the following equations:

$$
\begin{gathered}
a_{T+t}=a_{T}+r t \\
v_{T+t}=v_{T}+a_{T} t+\frac{1}{2} r t^{2} \\
d v_{1}=d_{T}+v_{T} t+\frac{1}{2} a_{T} t^{2}+\frac{1}{6} r t^{3}
\end{gathered}
$$

12. Similarly, the acceleration, speed and distance at time $T+2 t$ are computed as per the following equations:

$$
\begin{gathered}
a_{T+2 t}=a_{T+t}+r t=a_{T}+2 r t \\
v_{T+2 t}=v_{T+t}+a_{T+t} t+\frac{1}{2} r t^{2}=\left(v_{T}+a_{T} t+\frac{1}{2} r t^{2}\right)+\left(a_{T}+r t\right) t+\frac{1}{2} r t^{2}=v_{T}+2 a_{T} t+2 r t^{2}
\end{gathered}
$$




$$
\begin{aligned}
& d v_{2}=d_{T}+v_{T+t} t+\frac{1}{2} a_{T+t} t^{2}+\frac{1}{6} r t^{3}=t\left(v_{T}+a_{T} t+\frac{1}{2} r t^{2}\right)+\frac{1}{2} t^{2}\left(a_{T}+r t\right)+\frac{1}{6} r t^{3} \\
& =d_{T}+v_{T} t+\frac{3}{2} a_{T} t^{2}+\frac{7}{6} r t^{3}
\end{aligned}
$$

13. The acceleration, speed and distance at time $T+3 t$ are also computed as per the following equations:

$$
\begin{gathered}
a_{T+3 t}=a_{T+2 t}+r t=a_{T}+3 r t \\
v_{T+3 t}=v_{T+2 t}+a_{T+2 t} t+\frac{1}{2} r t^{2}=\left(v_{T}+2 a_{T} t+2 r t^{2}\right)+\left(a_{T}+2 r t\right) t+\frac{1}{2} r t^{2}=v_{T}+3 a_{T} t+\frac{9}{2} r t^{2}
\end{gathered}
$$

$$
\begin{aligned}
& d v_{3}=d_{T}+v_{T+2 t} t+\frac{1}{2} a_{T+2 t} t^{2}+\frac{1}{6} r t^{3}=d_{T}+\left(v_{T}+2 a_{T} t+2 r t^{2}\right) t+\frac{1}{2} t^{2}\left(a_{T}+2 r t\right)+\frac{1}{6} r t^{3} \\
& =d_{T}+v_{T} t+\frac{5}{2} a_{T} t^{2}+\frac{19}{6} r t^{3}
\end{aligned}
$$

14. The initial speed, $v_{T}$, the initial acceleration, $a_{T}$, and the rate of change in acceleration, $r$, can all be computed by solving equations $3.10,3.13$ and 3.16 together and they were found to be as follows (where $d v_{1}, d v_{2}$ and $d v_{3}$ are calculated from equations $3.1,3.2$ and 3.3 , respectively):

$$
\begin{aligned}
& v_{T}=\frac{\frac{11}{6} d v_{1}-\frac{7}{6} d v_{2}+\frac{1}{3} d v_{3}}{t} \\
& a_{T}=\frac{-2 d v_{1}+3 d v_{2}-d v_{3}}{t^{2}}
\end{aligned}
$$




$$
r=\frac{d v_{1}-2 d v_{2}+d v_{3}}{t^{3}}
$$

15. From Figure 3.14, the angles $\alpha$ and $\beta$ can be determined from the following equations:

$$
\begin{aligned}
& \sin \alpha=\frac{d_{3}}{d_{v 3}} \sin \left(\theta_{3}-\theta_{4}\right) \\
& \sin \beta=\sin \left(180^{\circ}-\beta\right)=\sin \alpha=\frac{d_{3}}{d_{v 3}} \sin \left(\theta_{3}-\theta_{4}\right)
\end{aligned}
$$

16. The offset between the bullet vehicle and the target vehicle, $w_{f}$, can be computed by the following equation (where the mean value has been taken to reduce the probability of reading errors):

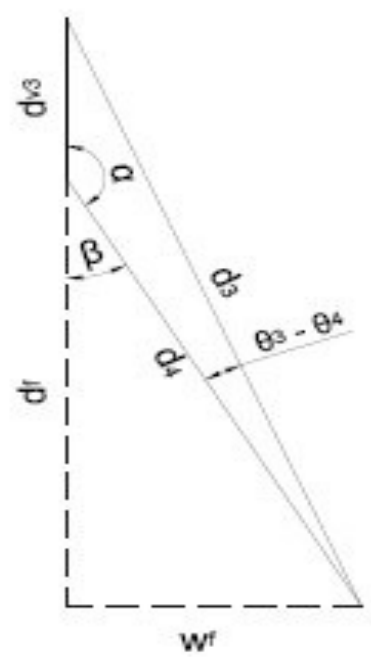

Figure 3.14 Calculating the distance $d_{f}$ and the offset $w_{f}$ 


$$
w_{f}=\frac{\sum_{n=1}^{3} d_{n+1} \sin \beta_{n+1}}{3}=\frac{\sum_{n=1}^{3} d_{n+1} \frac{d_{n}}{d_{v n}} \sin \left(\theta_{n}-\theta_{n+1}\right)}{3}=\frac{1}{3} \sum_{n=1}^{3} \frac{d_{n} d_{n+1} \sin \left(\theta_{n}-\theta_{n+1}\right)}{\sqrt{d_{n}^{2}+d_{n+1}^{2}-2 d_{n} d_{n+1} \cos \left(\theta_{n}-\theta_{n+1}\right)}}
$$

17. The distance from the bullet vehicle (at time $T+3 t$ ) to the intersection, $d_{f}$, can also be calculated as follows:

$$
d_{f}=\sqrt{d_{4}^{2}-w_{f}^{2}}
$$

18. The distance from the bullet vehicle (at time $T+3 t$ ) to the point of collision (shown in Figure 1.1), $d_{f}$, equals the distance to the intersection, $d_{f}$, minus a correction factor, $C_{L}$, for the number of lanes of the minor road that should be crossed by the target vehicle to reach the point of collision. There is uncertainty in determining that correction factor as it depends on the number of lanes of the minor road as well as the lane width and the median width. A conservative approach is by assuming that the minor road has three lanes on each direction with lane width $3.60 \mathrm{~m}$ and median width $4.00 \mathrm{~m}$ (the maximum typical lane width and median width in practice for arterials). In this case, the correction factor, $C_{L}$, will be $14.80 \mathrm{~m}$. Applying this correction factor will result in underestimating the time required for the bullet vehicle to reach the point of collision, tbullet, by less than 0.7 seconds (for typical running speed of $60 \mathrm{~km} / \mathrm{h}$ ) in case if the minor road has number of lanes, lane width or median width that are less than the maximum assumed values. This means that in some cases 
the proposed system will be more conservative by less than 0.7 seconds, which is insignificant and may not cause nuisance to the driver of the equipped vehicle.

19. The time required for the bullet vehicle to reach the point of collision, $t_{\text {bullet, }}$ is the smallest positive real root for the following polynomial equation that is based on Equation 3.7 (where $d_{f}$ is calculated from Equation 3.23, $v_{T+3 t}$ is calculated from Equation 3.15, $a_{T+3 t}$ is calculated from Equation 3.14, and $r$ is calculated from Equation 3.19):

$$
d_{f}=v_{T+3 t} t_{\text {bullet }}+\frac{1}{2} a_{T+3 t} t_{\text {bullet }}^{2}+\frac{1}{6} r t_{\text {bullet }}^{3}
$$

If the above polynomial equation returns no positive roots, the system should trigger a 'safe' signal as this indicates that the approaching vehicle is decelerating for a full a stop before reaching the intersection. If more than one positive root were returned, the smaller one should be considered by the system for further calculations as the larger one represents the imaginary mathematical case when the approaching vehicle is decelerating for a full stop at a point past the intersection and it mathematically reverses the direction of its velocity to the opposite direction (negative velocity) so that it mathematically reaches the same intersection again. 


\subsection{Calculating $t_{\text {target }}$}

As was discussed in Chapter 2, the total time required for the driver of the target vehicle to make a left turn, $t_{\text {target, }}$ is the sum of the following two times:

- Driver's perception-reaction time $\left(t_{1}\right)$, which is the time required for the driver to perceive the 'safe' signal and take appropriate action (activate throttle); and

- Vehicle's travel time $\left(t_{2}\right)$, which is the time required to accelerate the vehicle and to clear the path of the approaching bullet vehicle. This includes the time required to traverse the offset distance between the target vehicle and the bullet vehicle in addition to the length of the target vehicle itself. A correction factor, $C_{W}$, should also be added that equals the distance between the 'reflective' point (or the centre of a cluster of reflective points) in the approaching vehicle detected by the detection sensor and the far edge of the approaching vehicle that should be passed by the equipped vehicle to ensure safe departure. The reflective point detected by the sensor should be determined based on the technical specifications of the detection sensor used. If the reflective point is located at the near edge of the approaching vehicle, the correction factor, $C_{W}$, should equal to a full width of a passenger car, which is typically $2.13 \mathrm{~m}$ (AASHTO 2001); if the reflective point is located at the centre of the approaching vehicle, the correction factor, $C_{W}$, should equal to a half width of a passenger car, and if the reflective point is located at the far edge of the approaching vehicle, the correction factor, $C_{W}$, should equal to zero (no correction factor added). 


\subsubsection{Perception-reaction Time $\left(t_{1}\right)$}

It should be noted that the driver's perception-reaction time, when a collision warning system is installed, should be different from that when no collision warning system is installed. If a collision warning system is installed, the perception-reaction time for the driver is the time required to perceive the message, from the collision warning system, and to react accordingly by activating throttle. On the other hand, if a collision warning system is not installed, the driver has to perceive the whole situation, analyse it, make a decision and take appropriate action by activating the throttle.

Theoretically, it is expected that the perception-reaction time if a collision warning system is installed should be less than that if no collision warning system is installed due to the fewer tasks expected from the driver (no analysis or decision-making required). This expectation is subject to many factors, including reliability of the warning system and driver's familiarity with it. Actually, if the system is not reliable or if the driver is not familiar with it, the perception-reaction time may be much longer and likely to exceed that if no warning system is installed due to increased driver's mental load to verify the message given by the system by using his/her own judgement. Based on that, a dedicated chapter will be introduced to calibrate a model that estimates driver's perception-reaction time $\left(t_{1}\right)$ as precisely as possible since this is a key factor in the reliability of the system. 


\subsubsection{Vehicle Travel Time $\left(t_{2}\right)$}

A key issue in computing the anticipated travel time for the target vehicle, $t_{2}$, is to select the proper time-acceleration profile. The maximum acceleration of the target vehicle is based on its size, weight and engine power. The driver's desired acceleration of the target vehicle is less than the maximum acceleration provided by the mechanical characteristics of the target vehicle and it depends on other factors related to driver's personality and his/her perception for the situation (e.g., the location and the speed of the nearest approaching vehicle).

Different models were developed to represent the time-acceleration profile. The simplest model is by assuming a constant acceleration throughout the departure movement by using the following simple equations:

$$
\begin{aligned}
& a_{v}=\text { constant } \\
& a_{d}=a_{v} \times c_{d} \\
& V_{t}=V_{0}+a_{d} \times t \\
& d_{t}=V_{0} \times t+0.5 \times a_{d} \times t^{2}
\end{aligned}
$$

where $a_{v}$ is the maximum acceleration rate that can be provided by the mechanical characteristics of the host vehicle, $c_{d}$ is the driver's correction factor, $a_{d}$ is the acceleration rate selected by the driver averaged throughout the departure movement, $V_{t}$ is the speed of the host vehicle at time $t, V_{0}$ is the initial speed of the 
host vehicle (typically $0 \mathrm{~m} / \mathrm{sec}$ ) and $d_{t}$ is the distance traveled by the host vehicle at time $t$.

The above constant acceleration model may not be realistic reflection of vehicle acceleration as it was found that most vehicles accelerate more at lower speeds and less at higher speeds; and therefore, the linear decay acceleration model (Drew 1968; Long 2000) was developed based on the observation that the acceleration is inversely proportional to the speed as per the following equation:

$$
a_{t}=a_{v}\left(1-\frac{v_{t}}{v_{e}}\right)
$$

where $a_{t}$ is the acceleration rate at time $t ; a_{v}$ is the maximum acceleration rate provided by the mechanical characteristics of the vehicle at the start of its movement; $v_{t}$ is the speed of the vehicle at time $t$; and $v_{e}$ is the equilibrium speed (the crawl speed) where the acceleration decreases to zero, which also depends on the mechanical characteristics of the vehicle. By integrating Equation 3.29, the following two equations compute speed, $v_{t}$, and distance, $d_{t}$, at any time $t$ :

$$
\begin{array}{r}
v_{t}=a_{v} v_{e}\left(1-e^{-\frac{a_{v}}{v_{e}} t}\right)+v_{0} e^{-\frac{a_{v}}{v_{e}} t} \\
d_{t}=a_{v} v_{e} t-\frac{v_{e}^{2}}{a_{v}}\left(1-e^{-\frac{a_{v}}{v_{e}} t}\right)+\frac{v_{0} v_{e}}{a_{v}}\left(1-e^{-\frac{a_{v}}{v_{e}} t}\right)
\end{array}
$$


The driver's desired acceleration, $a_{d}$, is based on driver's characteristics as well as on vehicle performance. The driver's correction factor for vehicle acceleration, $C_{d}$, will be discussed in Chapter 5 and it represents a correction factor that should be applied to the rate of vehicle acceleration to account for driver's personal characteristics and his/her own perception for the situation (e.g., the location and speed of the nearest approaching vehicle). This can be represented by the following equation:

$$
a_{d}=C_{d} a_{v}
$$

The acceleration, $a_{t}$, speed, $v_{t}$, and distance, $d_{t}$, at any time $t$ can be computed from the following equations:

$$
\begin{aligned}
& a_{t}=a_{d}\left(1-\frac{v_{t}}{v_{e}}\right) \\
& v_{t}=a_{v} v_{e}\left(1-e^{-\frac{a_{d}}{v_{e}} t}\right)+v_{0} e^{-\frac{a_{d}}{v_{e}} t} \\
& d_{t}=a_{v} v_{e} t-\frac{v_{e}^{2}}{a_{d}}\left(1-e^{-\frac{a_{d}}{v_{e}} t}\right)+\frac{v_{0} v_{e}}{a_{d}}\left(1-e^{-\frac{a_{d}}{v_{e}} t}\right)
\end{aligned}
$$

To compute the vehicle's travel time, $t_{2}$, the total distance to be crossed by the target vehicle, $S$, is first computed, which is the sum of the offset, $w_{f}$, the length of the 
target vehicle, $L$, and the correction factor, $C_{W}$, for the distance between the 'reflective' point (or the centre of a cluster of reflective points) in the approaching vehicle detected by the detection sensor and the far edge of the approaching vehicle that should be passed by the equipped vehicle to ensure safe departure. The equation for calculating the distance $S$ is as follows:

$$
S=w_{f}+L+C_{W}
$$

where $S$ is the total distance to be crossed; $w_{f}$ is the side offset between the target vehicle and the bullet vehicle as was calculated from Equation 3.22; and $L$ is the length of the target vehicle, which can be pre-input to the algorithm as this is the length of the vehicle that the system will be mounted to.

The correction factor, $C_{W}$, depends on the location of the reflective point (or the centre of a cluster of reflective points) that is detected by the sensor, which depends on the technical specifications of the detection sensor used. If the reflective point is located at the near edge of the approaching vehicle, the correction factor, $C_{W}$, should equal to a full width of a passenger car, which is typically $2.13 \mathrm{~m}$ (AASHTO 2001); if the reflective point is located at the centre of the approaching vehicle, the correction factor, $C_{W}$, should equal to a half width of a passenger car, and if the reflective point is located at the far edge of the approaching vehicle, the correction factor, $C_{W}$, should equal to zero (no correction factor added). The time required for the crossing, $t_{2}$, is computed from Equation 3.35 where the initial speed, $v_{0}$, is set to zero (vehicle starts from stop) and the only unknown variable is the crossing time, $t_{2}$. 


\subsubsection{Total Time $\left(t_{\text {Target }}\right)$}

Finally, the total time required for the target vehicle to perform left turn, $t_{\text {target, }}$ is calculated by the following simple formula:

$$
t_{t \arg e t}=t_{1}+t_{2}
$$

where

$$
\begin{aligned}
& t_{\text {target }}=\text { the time required for the target vehicle to perform the left turn } \\
& \text { movement; } \\
& t_{1}=\text { driver's perception-reaction time; and } \\
& t_{2}=\text { crossing time }
\end{aligned}
$$

\subsection{Decision-Making Process}

The final step of the algorithm is to compare the time required for the bullet vehicle to reach the intersection, $t_{\text {bullet}}$, with the time required for the target vehicle to depart the intersection, $t_{\text {target }}$ A factor of safety may be added to represent a minimum gap between the target and bullet vehicles. It was found from earlier research (Ragland et al. 2005) that the majority of left-turning drivers feel more comfortable when the clear gap between their turning vehicle and other vehicles approaching on the opposite traffic lanes is 2 seconds when they make their left-turn departure movement; and therefore, to keep the proposed system consistent with 
driver's normal behaviour (so that the system does not cause nuisance to the driver), the following criterion is used:

$$
t_{\text {bullet }}-t_{\text {target }}>2.0 \mathrm{sec} \text {. }
$$

If the above criterion is met for all detected vehicles, a 'safe' message is displayed to the driver; otherwise, the 'not safe' message continues to be displayed until the above criterion is met. 


\section{CHAPTER 4: ALGORITHM DESIGN FOR SEMI-CONTROLLED INTERSECTIONS}

This chapter describes the architecture for the proposed algorithm used to warn against possible collisions at semi-controlled (e.g., two-way stop controlled) intersections and the method used to estimate the time required for any detected bullet vehicle to reach the intersection and also the method used to estimate the time required for the target vehicle to complete the movement required (either to turn right, turn left, or pass through). The chapter also covers the methodology used to compare the two values and make a decision to give an appropriate warning message to prevent any possible collision.

It should be noted that in order for the system to detect the approaching vehicles, the intersection should have adequate sight distances as was suggested by design guides and earlier research findings (Easa et al., 2004). Therefore, the proposed system should not be considered as an aid for drivers in situations of insufficient intersection sight distance.

As was previously shown in Chapter 1 , the proposed algorithm is not designed for cases when an intersection collision is resulted from driver's violation for traffic bylaws. An example for that is at a signalised intersection where the driver of a vehicle tries to pass through the intersection while the traffic signal is red (usually at the start of the red phase), as was shown in Figure 1.2(b). In this case, that vehicle would be either a target or a bullet, and any possible collision would be merely resulted from that driver's aggressive driving attitudes rather than from his/her misjudgement, 
giving that the cycle length for the traffic signal is properly designed and provides adequate amber phase that does not cause dilemma for the driver. Furthermore, most traffic agencies provide an all-red phase to reduce the probability of the occurrence of this type of collisions.

Based on the above facts, a collision warning system will not be useful in reducing this particular type of collisions that are based on driver's violation for the law; therefore there is no need for any collision warning system to address this situation because the driver is already informed by the traffic control device and it is his/her aggressive driving attitudes, not the lack of information, that lead to this type of collisions. Proper law enforcement measures, such as red-light cameras, will be more effective to address this particular type of collisions.

\subsection{Algorithm Architecture}

The proposed algorithm utilizes a pair of detection sensors (either radar sensors similar to the ICA and ICAV systems, or laser scanners similar to the INTERSAFE system). One sensor is installed at each of the two front corners of the turning (target) vehicle, as shown in Figure 4.1. Using the two detection sensors, along with the same processing unit and driver-vehicle interface used for left-turn movement (shown in Chapter 3), the algorithm follows procedures, for each sensor, similar to those discussed for the left-turn shown in Figure 3.2. 
The objective is to determine whether a 'safe' message should be displayed to the driver of the turning (target) vehicle. The procedures are as follows (Dabbour and Easa 2008):

1. Once the brakes are activated for a full stop and the detection sensors detect cross-traffic vehicles, as in a typical semi-controlled intersection situation, the system displays a warning message by default. Similar to the left-turn situation, the warning message could be visual, auditory, haptic, or a combination of two or more methods. The warning message is not deactivated until the algorithm confirms that a safe departure is available;

2. The system estimates the time required for each vehicle detected in the crosstraffic stream to reach the intersection, $t_{\text {bullet; }}$

3. The system estimates the time required for the turning vehicle to safely depart the intersection, $t_{\text {target; }}$

4. If the time required for the cross-traffic vehicle to reach the intersection, $t_{\text {bullet, }}$ was found to be greater than the time required to complete departure movement, $t_{\text {target, }}$ by more than the factor of safety used, a 'safe' message will be displayed to the driver of the turning vehicle to allow him/her to start the departure movement; or

5. If the time required for the cross-traffic vehicle to reach the intersection, $t_{\text {bullet }}$, was found to be greater than the time required for safe departure, $t_{\text {target }}$, by less than the factor of safety used, the warning message will continue to be active and the system will repeat the above algorithm until the criterion shown in the previous step is met for both detection sensors. 


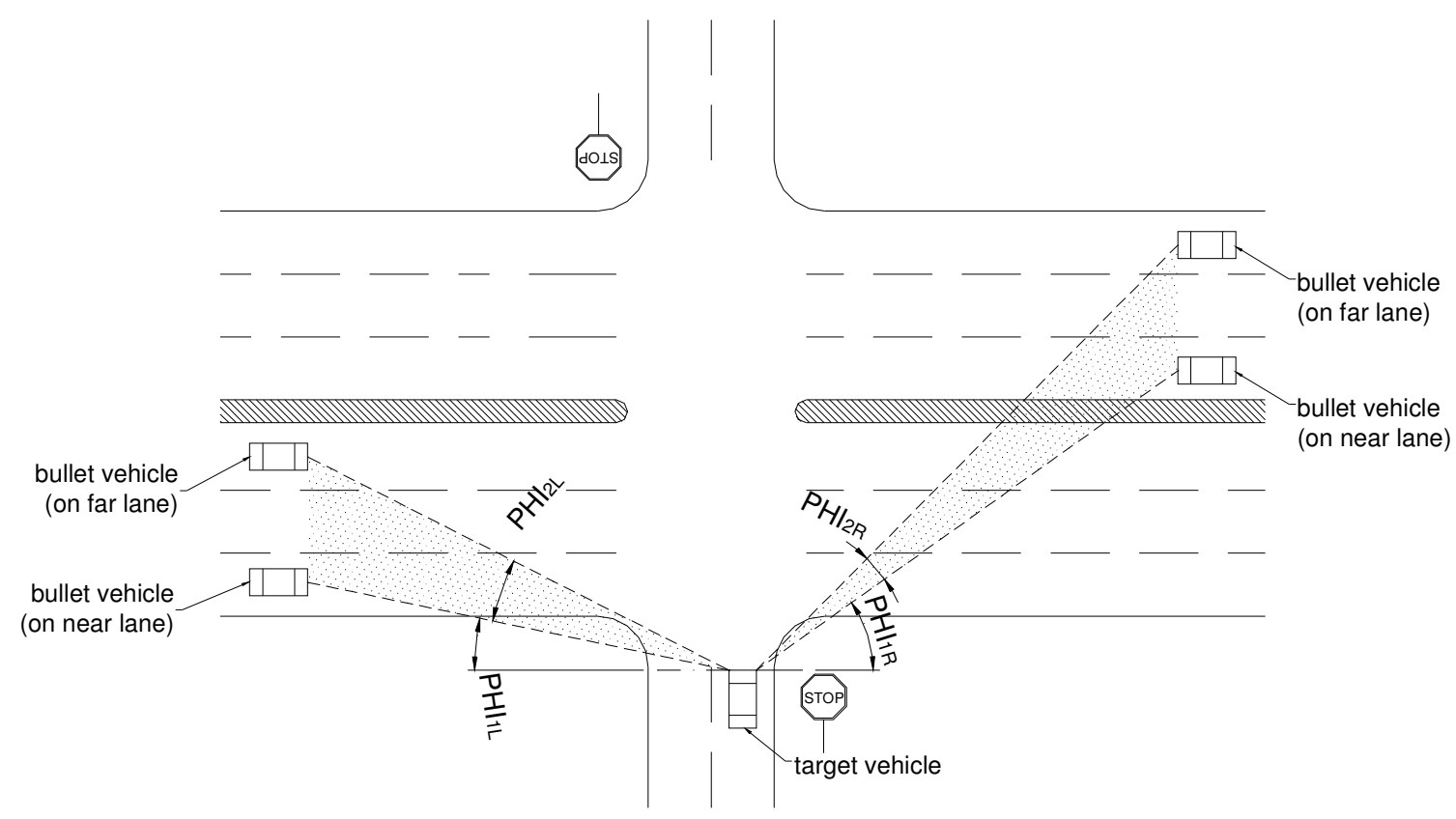

Figure 4.1 Configuration of the proposed detection sensors

Similar to the left-turn situation, the algorithm is capable of recognizing different approaching vehicles on different lanes and the above procedures are followed for each vehicle on each lane and no 'safe' message is activated until all lanes are clear of approaching vehicles that could collide with the departing vehicle. Similar to the left-turn situation, Kalman filter is also used for vehicle tracking as per the methodology described in Chapter 2.

\subsection{Specifications of Detection Sensors}

Similar to the left-turn situation, the detection sensors send detection beams every time interval, $t$, to scan cross-traffic lanes. The width of the radar beam is 
designed so that the angle between the outer edge of the beam and the face plane of the vehicle, $P H I_{1 L}$ or $P H I_{1 R}$ is to detect an approaching vehicle, either from the left direction or from the right direction, within a safe intersection site distance for a typical semi-controlled intersection. For instance, the intersection site distance for a two-way stop-controlled (TWSC) intersection is explained in case B in Chapter 9 of the geometric design guide (AASHTO 2001). From the guide, the intersection sight distance, ISD, along the major road is determined by:

$$
I S D=0.278 V_{\text {major }} t_{g}
$$

where:

- $V_{\text {major }}$ is the design speed of the major road $(\mathrm{km} / \mathrm{h})$; and

$\circ t_{g}$ is the time gap for the vehicle on the minor road to enter the major road (sec.).

From Exhibit 9-55 (AASHTO 2001), the intersection sight distance for a design speed of $70 \mathrm{~km} / \mathrm{h}$ is $150 \mathrm{~m}$. The design speed of $70 \mathrm{~km} / \mathrm{h}$ has been selected as it is the maximum design speed for a major road in an unsignalised intersection. It should be noted that the intersection sight distance shown above is a conservative value suggested by the design aids (AASHTO 2001; TAC 1999) and it is used in this research for the purpose of selecting the appropriate opening angle for the detection sensors used. The actual distance between the bullet vehicle and the intersection is 
calculated on the basis of the actual location of the bullet vehicle as well as its speed and acceleration rate, as will be explained herein after in this chapter.

For vehicles approaching from the left side (as shown in Figure 4.1), the angle with the plane face of the vehicle is $0^{\circ}$ (with a conservative assumption of no setback). The opening angle has a tan of 13.5/150 (assuming maximum 3 lanes), which yields an opening angle of $6^{\circ}$. For vehicles approaching from the right side (as shown in Figure 4.1), another detection sensor is used with angle with the plane of the vehicle has tan of approximately $3.5 / 150$, which yields an angle of $1.3^{\circ}$ (assuming one lane on each direction on the major road and no setback). The opening angle is set to detect an approaching vehicle on the far lane assuming three lanes on each direction on the major road and setback of $3.0 \mathrm{~m}$ (as a more conservative assumption for the calculation of the opening angle). In that case, the angle tan is approximately 27/150 (assuming 6 lanes, 3 m setback and 3 m median), which yields an opening angle of $\left(11^{\circ}-1.3^{\circ}=9.7^{\circ}\right)$.

\subsection{Algorithm Design}

Unlike the methodology used for left-turn movement that utilizes one detection sensor (as was shown in Chapter 3), the methodology for departing a minor road utilizes two detection sensors; and therefore two different algorithms should be followed (one algorithm for each detection sensor) where each algorithm has its procedures that are comparable to the methodology used for left-turn movement 
shown in Chapter 3. No 'safe' message is displayed to the driver until the algorithm confirms that the time required for every approaching vehicle to reach the intersection is larger than the time required for the equipped vehicle to depart the intersection.

The time anticipated for the bullet vehicle to reach the intersection, $t_{\text {bullet, }}$ as well as the time required for the host target vehicle to depart the intersection, $t_{\text {target, }}$, are both affected by the intended direction of the host target vehicle as well as the direction where the bullet vehicle is approaching from, as explained in the following three cases that are also shown in Figure 4.2:

- The first case is when the paths of the target and bullet vehicles are parallel to each other with no conflict (as shown in Figure 4.2a); an example for that case is when the target vehicle is turning right while the bullet vehicle is approaching from the right. A 'safe' message should be displayed to the driver of the target vehicle (unless another object is detected by any of the two detection sensors).

- The second case is when the paths of the target and bullet vehicles are perpendicular to each other (as shown in Figure 4.2b); that case particularly occurs when the target vehicle is turning left while the bullet vehicle is approaching from the left or when the target vehicle is going straight while the bullet vehicle is approaching from either the left or the right. In that case, the algorithm calculates the anticipated time for the bullet vehicle to reach the intersection and compares it to the required time for the target vehicle to clear the path of the bullet vehicle. This case will be discussed in detail in the subsequent sections. 
- The third case is when both the target and bullet vehicles are traveling on the same lane in the same direction (as shown in Figure 4.2c); that case particularly occurs when the target vehicle is turning right while the bullet vehicle is approaching from the left on the same lane, or when the target vehicle is turning left while the bullet vehicle is approaching from the right on the same lane. In that case, the algorithm calculates the time required for the target vehicle to accelerate to $70 \%$ of the speed of the bullet vehicle, which is the criterion recommended by the design guides and earlier research (AAHTO 2001). The algorithm also determines the distance from the intersection to the conflict point where the target vehicle completes its acceleration to $70 \%$ of the speed of the bullet vehicle. The algorithm then computes the anticipated time for the bullet vehicle to reach that conflict point and compares it to the required time for the target vehicle to reach the same point. This case will also be discussed in detail in the subsequent sections.

\subsubsection{Determining Bullet Vehicle Location}

A schematic illustration is shown in Figure 4.3 to depict the methodology used to compute the time required for the bullet vehicle to reach the intersection, $t_{\text {bullet, }}$ for the case when the paths of the bullet and target vehicles are perpendicular to each other, which can be summarised as follows:

1. A detection beam is generated, from each of the two detection sensors, at time $T$ to scan all cross-traffic lanes. If no object is detected a 'safe' signal is 
displayed to the driver. Otherwise, the nearest object detected, vehicle $A$, is registered at distance $d_{1}$ and azimuth angle $\theta_{1}$ (measured from the face plane of the vehicle) where polar coordinates are used with the origin point to coincide with the location of the detection sensor, as shown in Figure 4.4.

2. Another radar beam is generated at time $T+t$, where $t$ is the time interval for the detection sensor, and the new location of vehicle $A$ is registered at distance $d_{2}$ and azimuth angle $\theta_{2}$ where the same above polar coordinates are used, as shown in Figure 4.5.

3. If $d_{1}$ and $d_{2}$ were found to be equal, the algorithm concludes that the object is not moving (e.g., a tree or a building). A 'safe' message is displayed to the driver (unless another object is detected from any of the two detection sensors). It should be noted that generating a 'safe' signal is based on the assumption that the intersection is adequately designed for intersection sight distance as given by design guides (TAC 1999; AASHTO 2001).

4. If $d_{2}$ was found to be greater than $d_{1}$, the algorithm concludes that the object is moving away from the turning (target) vehicle and a 'safe' message is also displayed to the driver (unless another object is detected from any of the two detection sensors).

5. If $d_{2}$ was found to be less than $d_{1}$, the distance traversed by the approaching vehicle during the first time interval, $d_{v 1}$, is calculated as shown in Figure 4.6 using the following formula:

$$
d_{v 1}=\sqrt{d_{1}^{2}+d_{2}^{2}-2 d_{1} d_{2} \cos \left(\theta_{1}-\theta_{2}\right)}
$$


(a)
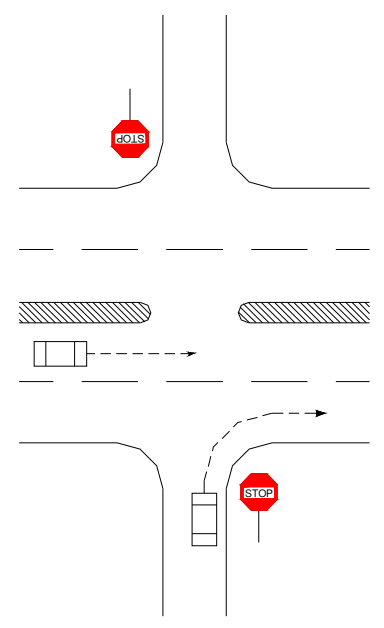

(b)

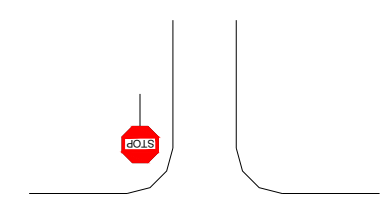

(b)

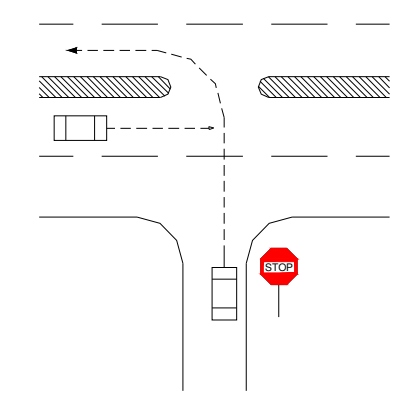

(c)
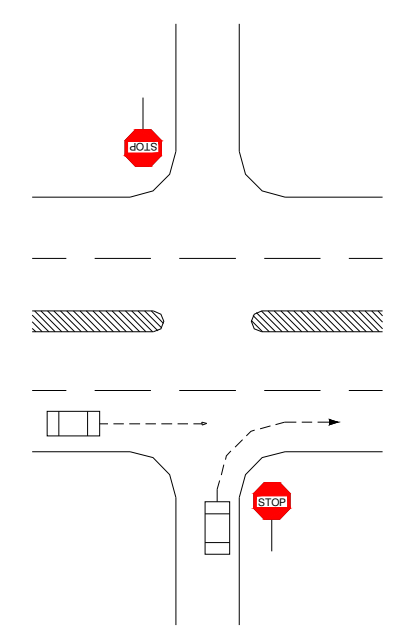
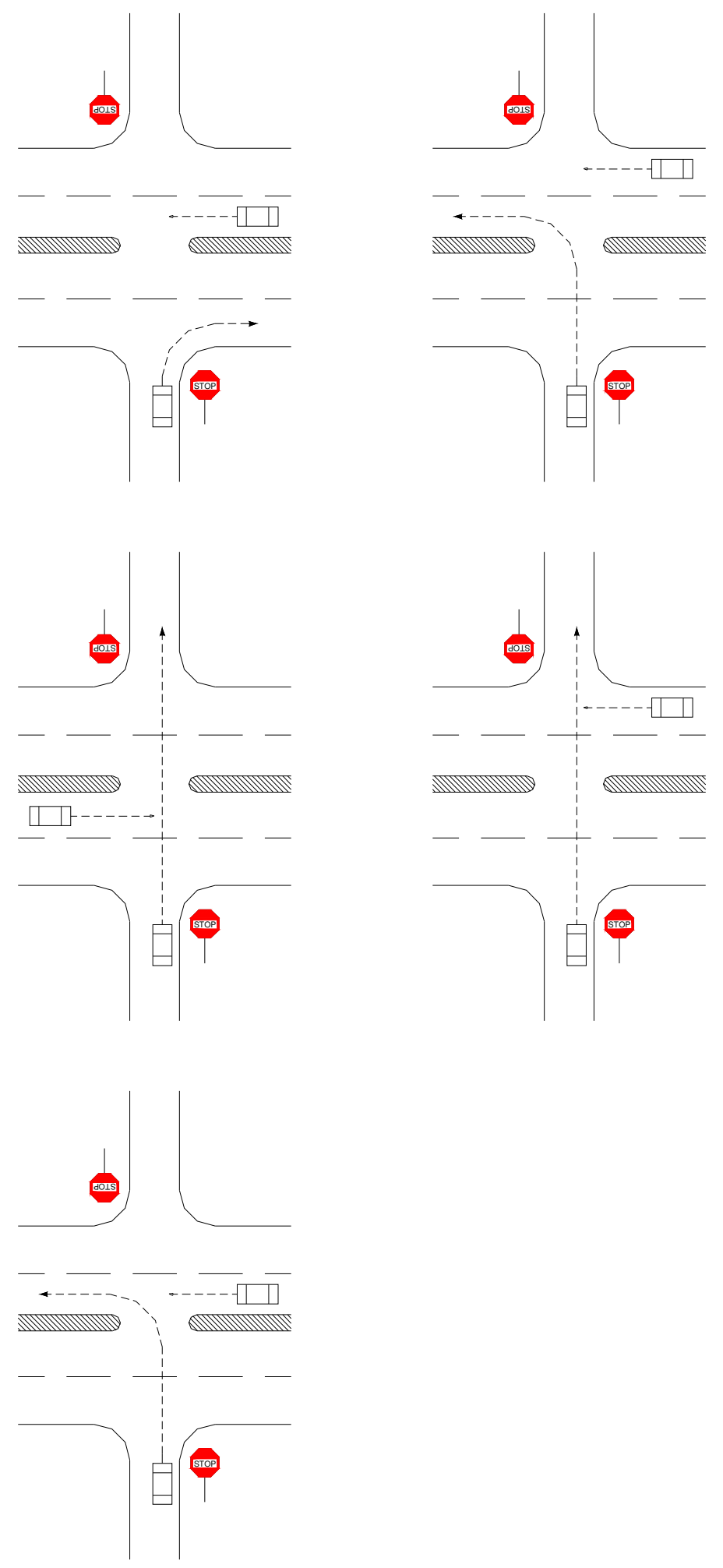

Figure 4.2 Conflict cases for a typical TWSC intersection 


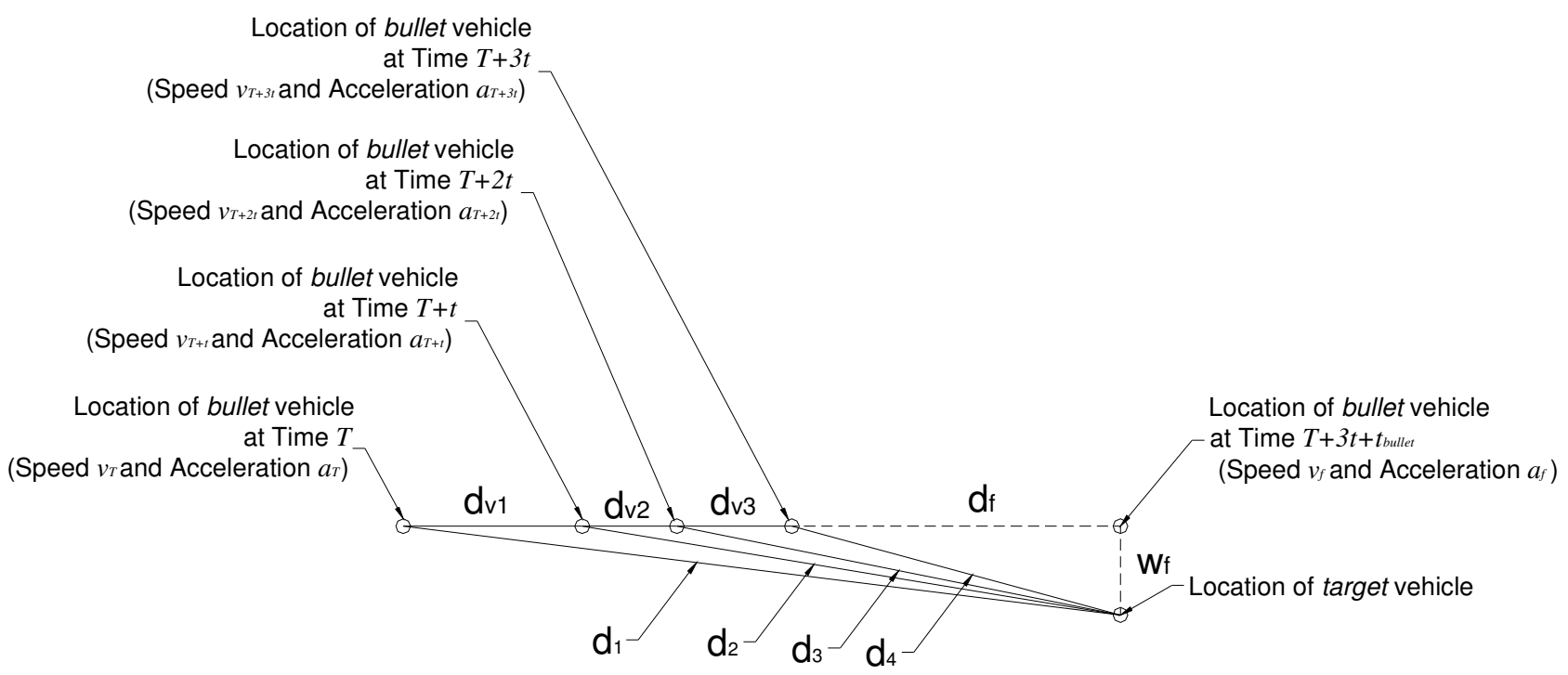

Figure 4.3 Schematic illustration of bullet vehicle locations at different time instances

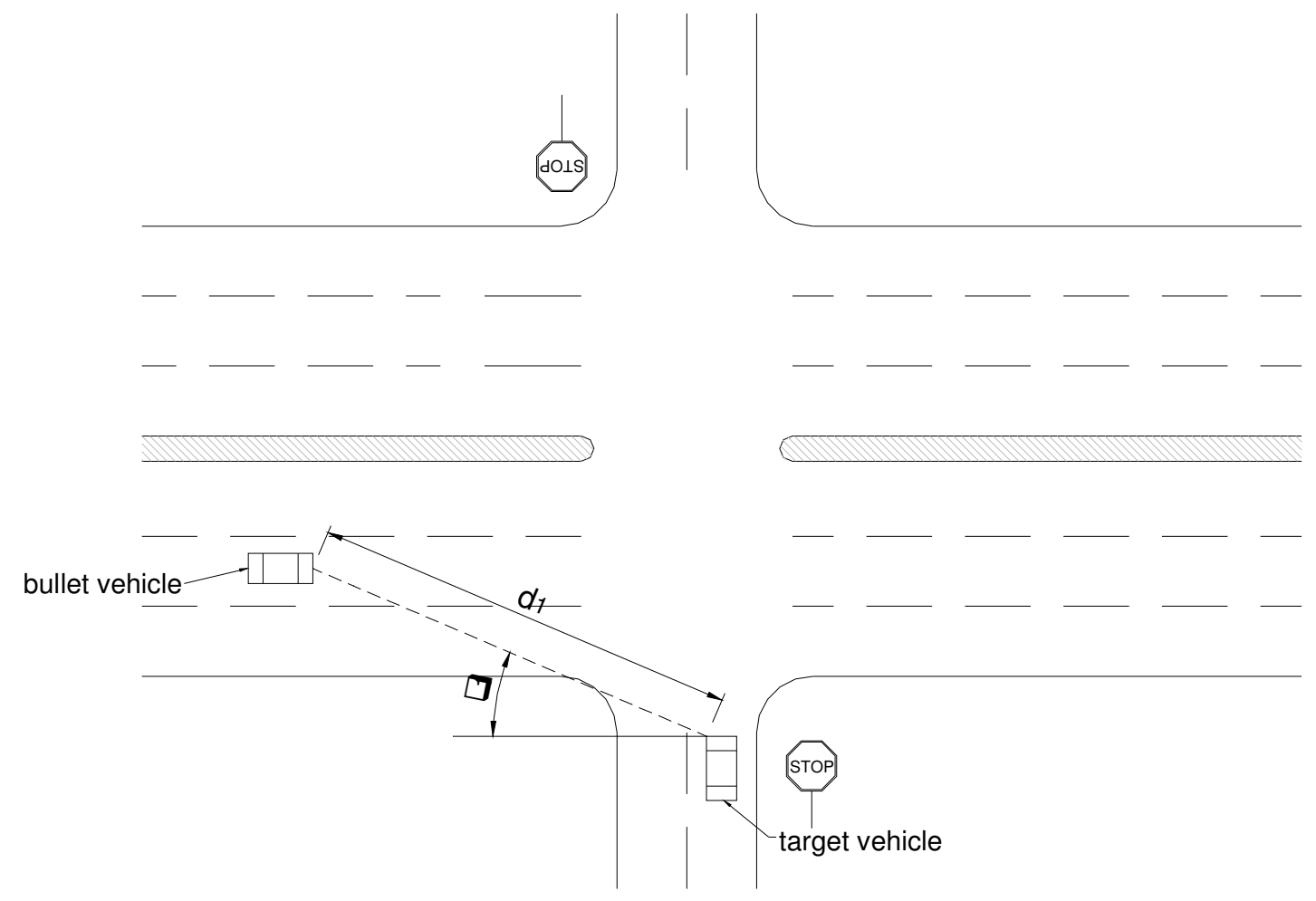

Figure 4.4 Measuring the range and azimuth angle at time $T$ 


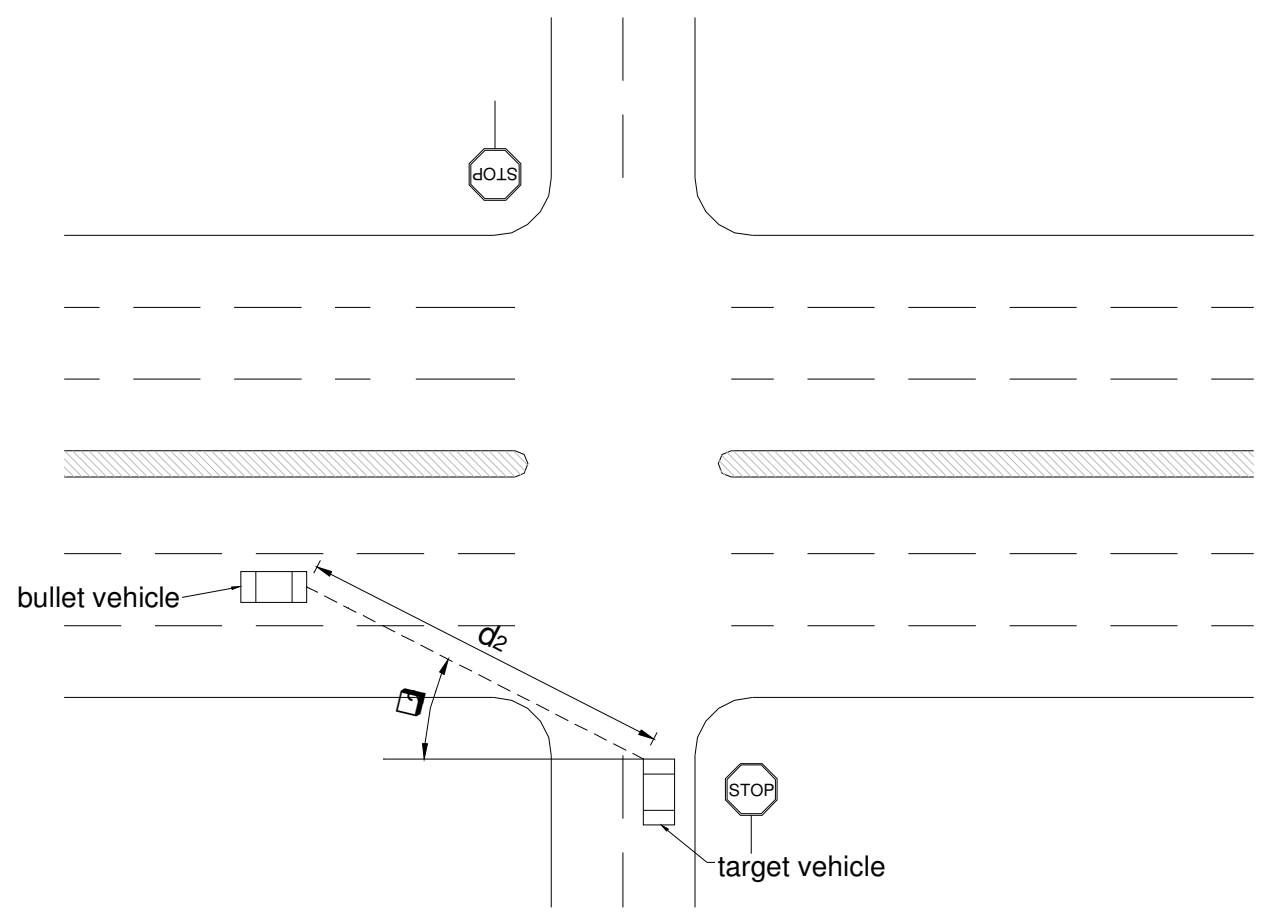

Figure 4.5 Measuring the range and azimuth angle at time $T+t$

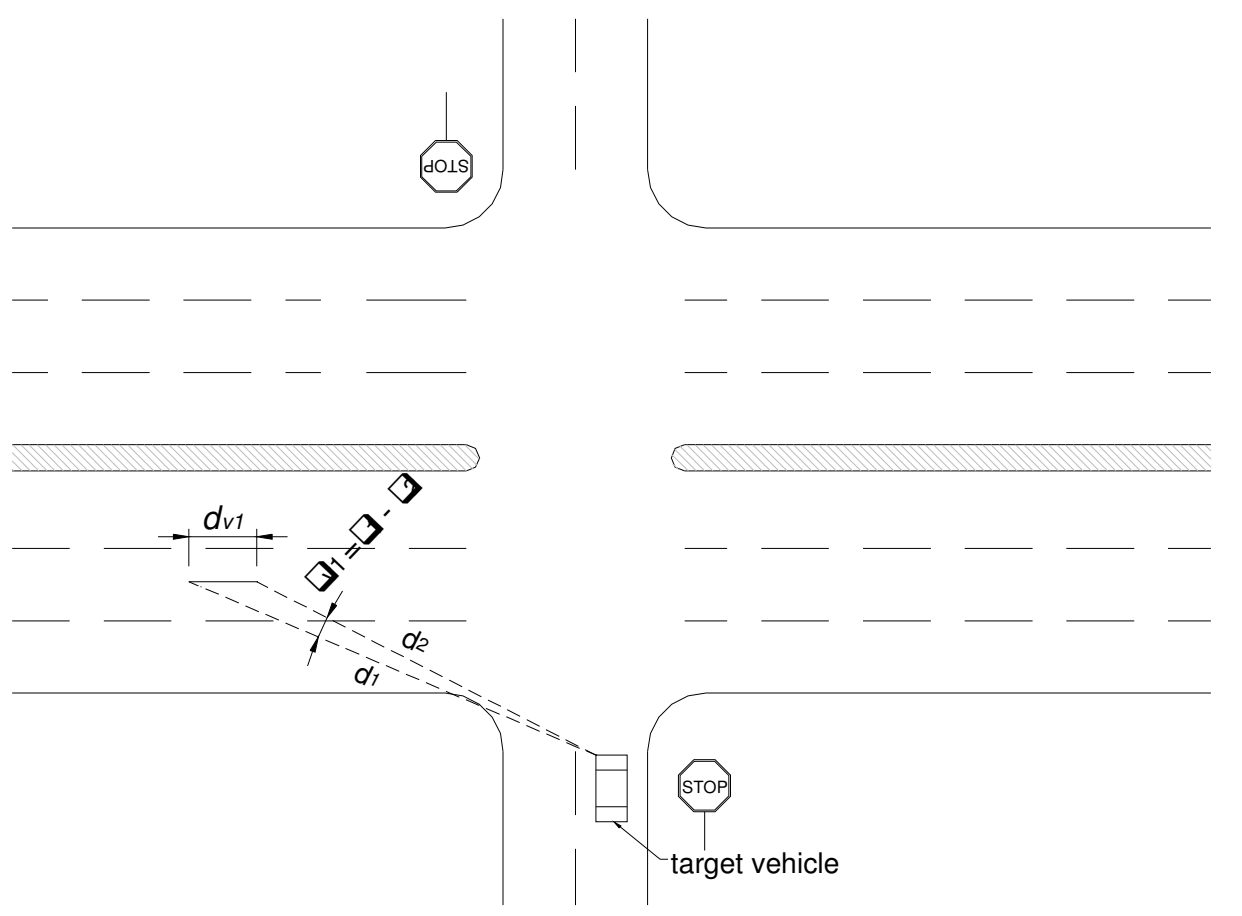

Figure 4.6 Calculating bullet vehicle displacement during first time interval 
6. A third radar beam is generated at time $T+2 t$ and the information for vehicle $A$ are recorded, including distance, $d_{3}$, and azimuth angle, $\theta_{3}$. The distance traversed by the vehicle during the second time interval, $d_{v 2}$, is calculated similar to calculating $d_{v 1}$ by using the following formula:

$$
d_{v 2}=\sqrt{d_{2}^{2}+d_{3}^{2}-2 d_{2} d_{3} \cos \left(\theta_{2}-\theta_{3}\right)}
$$

7. Similarly, a fourth radar beam is generated at time $T+3 t$. The distance, $d_{4}$, and azimuth angle, $\theta_{4}$, are both measured, and the distance traversed by the vehicle during the third time interval, $d_{v 3}$, is also calculated by using the following formula:

$$
d_{v 3}=\sqrt{d_{3}^{2}+d_{4}^{2}-2 d_{3} d_{4} \cos \left(\theta_{3}-\theta_{4}\right)}
$$

8. Similar to the left-turn case, and given the small time intervals used, the timeacceleration relationship can be assumed as a linear model with slope $r$, which is the rate of change in acceleration as represented by the following equation:

$$
\frac{d a}{d t}=r
$$


9. The acceleration, speed and distance at any time, $t$, can be computed using the following equations (where $a_{0}, v_{0}$ and $d_{0}$ are the initial acceleration, speed and distance, respectively):

$$
\begin{aligned}
& a_{t}=\int_{0}^{t} \frac{d a}{d t} d t=a_{T}+r t \\
& v_{t}=\int_{0}^{t} a_{t} d t=v_{T}+a_{T} t+\frac{1}{2} r t^{2} \\
& d_{t}=\int_{0}^{t} v_{t} d t=d_{T}+v_{T} t+\frac{1}{2} a_{T} t^{2}+\frac{1}{6} r t^{3}
\end{aligned}
$$

10. By applying the above three equations at different time intervals, the acceleration, speed and distance at time $T+t$ are computed as per the following equations:

$$
\begin{aligned}
& a_{T+t}=a_{T}+r t \\
& v_{T+t}=v_{T}+a_{T} t+\frac{1}{2} r t^{2} \\
& d v_{1}=d_{T}+v_{T} t+\frac{1}{2} a_{T} t^{2}+\frac{1}{6} r t^{3}
\end{aligned}
$$

11. Similarly, the acceleration, speed and distance at time $T+2 t$ are computed as per the following equations: 


$$
\begin{gathered}
a_{T+2 t}=a_{T+t}+r t=a_{T}+2 r t \\
v_{T+2 t}=v_{T+t}+a_{T+t} t+\frac{1}{2} r t^{2}=\left(v_{T}+a_{T} t+\frac{1}{2} r t^{2}\right)+\left(a_{T}+r t\right) t+\frac{1}{2} r t^{2}=v_{T}+2 a_{T} t+2 r t^{2} \\
d v_{2}=d_{T}+v_{T+t} t+\frac{1}{2} a_{T+t} t^{2}+\frac{1}{6} r t^{3} \\
=d_{T}+t\left(v_{T}+a_{T} t+\frac{1}{2} r t^{2}\right)+\frac{1}{2} t^{2}\left(a_{T}+r t\right)+\frac{1}{6} r t^{3}=v_{T} t+\frac{3}{2} a_{T} t^{2}+\frac{7}{6} r t^{3}
\end{gathered}
$$

12. The acceleration, speed and distance at time $T+3 t$ are also computed as per the following equations:

$$
\begin{gathered}
a_{T+3 t}=a_{T+2 t}+r t=a_{T}+3 r t \\
v_{T+3 t}=v_{T+2 t}+a_{T+2 t} t+\frac{1}{2} r t^{2}=\left(v_{T}+2 a_{T} t+2 r t^{2}\right)+\left(a_{T}+2 r t\right) t+\frac{1}{2} r t^{2}=v_{T}+3 a_{T} t+\frac{9}{2} r t^{2}
\end{gathered}
$$

$$
\begin{aligned}
& d v_{3}=d_{T}+v_{T+2 t} t+\frac{1}{2} a_{T+2 t} t^{2}+\frac{1}{6} r t^{3}=d_{T}+\left(v_{T}+2 a_{T} t+2 r t^{2}\right) t+\frac{1}{2} t^{2}\left(a_{T}+2 r t\right)+\frac{1}{6} r t^{3} \\
& =d_{T}+v_{T} t+\frac{5}{2} a_{T} t^{2}+\frac{19}{6} r t^{3}
\end{aligned}
$$

13. The initial speed, $v_{T}$, the initial acceleration, $a_{T}$, and the rate of change in acceleration, $r$, can all be computed by solving equations $4.11,4.14$ and 4.17 together and they were found to be as follows (where $d v_{1}, d v_{2}$ and $d v_{3}$ are calculated from equations $4.2,4.3$ and 4.4 , respectively): 


$$
\begin{aligned}
& v_{T}=\frac{\frac{11}{6} d v_{1}-\frac{7}{6} d v_{2}+\frac{1}{3} d v_{3}}{t} \\
& a_{T}=\frac{-2 d v_{1}+3 d v_{2}-d v_{3}}{t^{2}} \\
& r=\frac{d v_{1}-2 d v_{2}+d v_{3}}{t^{3}}
\end{aligned}
$$

14. From Figure 4.7, the angles $\alpha$ and $\beta$ can be computed from the following equations:

$$
\begin{aligned}
& \sin \alpha=\frac{d_{3}}{d_{v 3}} \sin \left(\theta_{3}-\theta_{4}\right) \\
& \sin \beta=\sin \left(180^{\circ}-\beta\right)=\sin \alpha=\frac{d_{3}}{d_{v 3}} \sin \left(\theta_{3}-\theta_{4}\right)
\end{aligned}
$$

15. The side offset between the bullet vehicle and the target vehicle, $w_{f}$, can be computed by the following equation (where the mean value has been taken to reduce the probability of reading errors):

$$
w_{f}=\frac{\sum_{n=1}^{3} d_{n+1} \sin \beta_{n+1}}{3}=\frac{\sum_{n=1}^{3} d_{n+1} \frac{d_{n}}{d_{v n}} \sin \left(\theta_{n}-\theta_{n+1}\right)}{3}=\frac{1}{3} \sum_{n=1}^{3} \frac{d_{n} d_{n+1} \sin \left(\theta_{n}-\theta_{n+1}\right)}{\sqrt{d_{n}^{2}+d_{n+1}^{2}-2 d_{n} d_{n+1} \cos \left(\theta_{n}-\theta_{n+1}\right)}}
$$




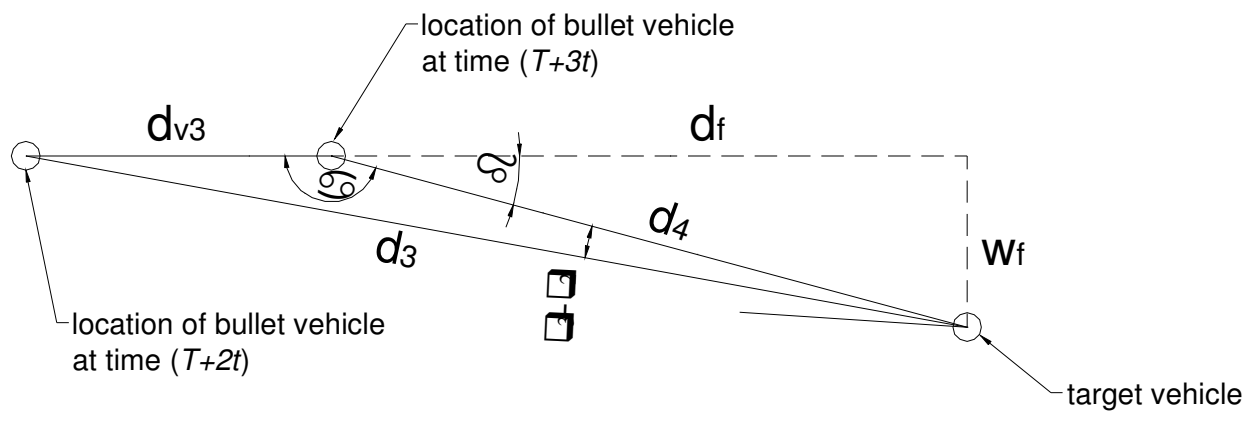

Figure 4.7 Calculating side offset and distance between bullet and target vehicles

16. The distance from the bullet vehicle (at time $T+3 t$ ) to the intersection, $d_{f}$, can also be computed as follows:

$$
d_{f}=\sqrt{d_{4}^{2}-w_{f}^{2}}
$$

17. Based on the conflict type between the bullet and target vehicles (either they have perpendicular paths or they are traveling on the same lane), the bullet time, $t_{\text {bullet, }}$ and target time, $t_{\text {trget }}$, can be computed as per one of the two cases explained below.

\subsubsection{Conflicting Vehicles have Perpendicular Paths}

When the bullet and target vehicles have perpendicular paths as shown in Figure $4.2 \mathrm{~b}$, the bullet and target times are computed in a similar manner to what was used for the left-turn situation. The bullet time is the smallest positive real root of the following polynomial equation (where $d_{f}$ is calculated from Equation $4.24, v_{T+3 t}$ is 
calculated from Equation 4.16, $a_{T+3 t}$ is calculated from Equation 4.15, and $r$ is calculated from Equation 4.20):

$$
d_{f}=v_{T+3 t} t_{\text {bullet }}+\frac{1}{2} a_{T+3 t} t_{\text {bullet }}^{2}+\frac{1}{6} r t_{\text {bullet }}^{3}
$$

Similar to the left-turn situation, if the above polynomial equation returns no positive roots, the system should trigger a 'safe' signal as this indicates that the approaching vehicle is decelerating for a full a stop before reaching the intersection. If more than one positive root were returned, the smaller one should be considered by the system for further calculations as the larger one represents the imaginary mathematical case when the approaching vehicle is decelerating for a full stop at a point past the intersection and it mathematically reverses the direction of its velocity to the opposite direction (negative velocity) so that it mathematically reaches the same intersection again.

It should also be noted that as a factor of safety and to keep the system consistent with driver's habits without causing nuisance, the bullet time calculated by the system should not be less than 7.5 seconds with adding another 0.5 seconds for each additional lane that should be crossed (where the number of lanes to be crossed is determined from the side offset calculated by Equation 4.23). This is based on the findings of design guides and previous studies (AASHTO 2001; Harwood et al. 1996) where it was found that a passenger car driver who is departing a minor road onto a two-lane major road in a TWSC 
intersection mentally needs the above minimum value for the total gap available for departure.

If the bullet time calculated by the system was found to be less than the minimum gap required, the system should not trigger a 'safe' signal even if the target time was found to be less than the bullet time calculated by the system. For instance, if the bullet time calculated by the system was found to be 6.0 seconds, the system should not trigger a 'safe' signal as the driver of the equipped vehicle will not feel comfortable to depart the intersection with an approaching vehicle that is too close, even if the required departure time was found to be less than the time required for the bullet vehicle to reach the intersection, which is 6.0 seconds. The system in that case would interfere with the driver's normal behaviour and may cause nuisance to the driver and result in losing its reliability. However, this additional condition may be eventually eliminated in a later stage after the driver gets used to the system and gains more confidence in it.

As for the target time, $t_{\text {target, }}$ it is computed as per the following methodology:

1. The driver's perception-reaction time, $t_{1}$, is computed from the statistical model that will be discussed later in Chapter 5 .

2. The vehicle's travel time, $t_{2}$, is computed using the following methodology:

a. The total distance to be crossed by the target vehicle, $S$, is the sum of the offset, $w_{f}$, the length of the target vehicle, $L$, and a correction factor, $C_{W}$, for the distance between the 'reflective' point (or the centre of a cluster of 
reflective points) in the approaching vehicle detected by the detection sensor and the far edge of the approaching vehicle that should be passed by the equipped vehicle to ensure safe departure, where the correction factor, $C_{W}$, assumes values that are similar to those in left-turn situation. The equation for calculating the distance $S$ is as follows::

$$
S=w_{f}+L+C_{W}
$$

b. The driver's desired acceleration, $a_{d}$, is calculated from the following equation (where the correction factor, $C_{d}$, is as will be computed from the statistical model that will be discussed later in Chapter 5 and $a_{v}$ is the maximum acceleration rate provided by the mechanical characteristics of the equipped target vehicle):

$$
a_{d}=C_{d} a_{v}
$$

c. The time required for crossing, $t_{2}$, is computed from the following equation where $v_{e}$ is the equilibrium (crawl) speed and the only unknown variable is the crossing time, $t_{2}$ :

$$
S=a_{d} v_{e} t_{2}-\frac{v_{e}^{2}}{a_{d}}\left(1-e^{-\frac{a_{d} t_{2}}{v_{e}}}\right)
$$


3. The total time required for the target vehicle to depart the intersection, $t_{\text {target }}$, is the sum of the driver's perception-reaction time and the vehicle's travel time as per the following equation:

$$
t_{\text {targ } e t}=t_{1}+t_{2}
$$

\subsubsection{Conflicting Vehicles Traveling on the Same Lane}

Where both the target and bullet vehicles are traveling on the same lane in the same direction (as shown in Figure 4.2c), the conflict point should be located somewhere between the intersection and point ' $\mathrm{B}$ ', which is the location where the target vehicle accelerates to $70 \%$ of the speed of the bullet vehicle.

The conflict scenario is explained in Figure 4.8 and can be summarised as follows:

1. At time $T+3 t$ : the target vehicle is still in a stop position while the bullet vehicle is traveling at speed $v_{T+3 t}$ and acceleration $a_{T+3 t}$, which are computed as per the following two equations, respectively:

$$
a_{T+3 t}=a_{T}+3 r t=\frac{-2 d v_{1}+3 d v_{2}-d v_{3}}{t^{2}}+3 t \frac{d v_{1}-2 d v_{2}+d v_{3}}{t^{3}}=\frac{d v_{1}-3 d v_{2}+2 d v_{3}}{t^{2}}
$$


(a) Time $T+3 t$
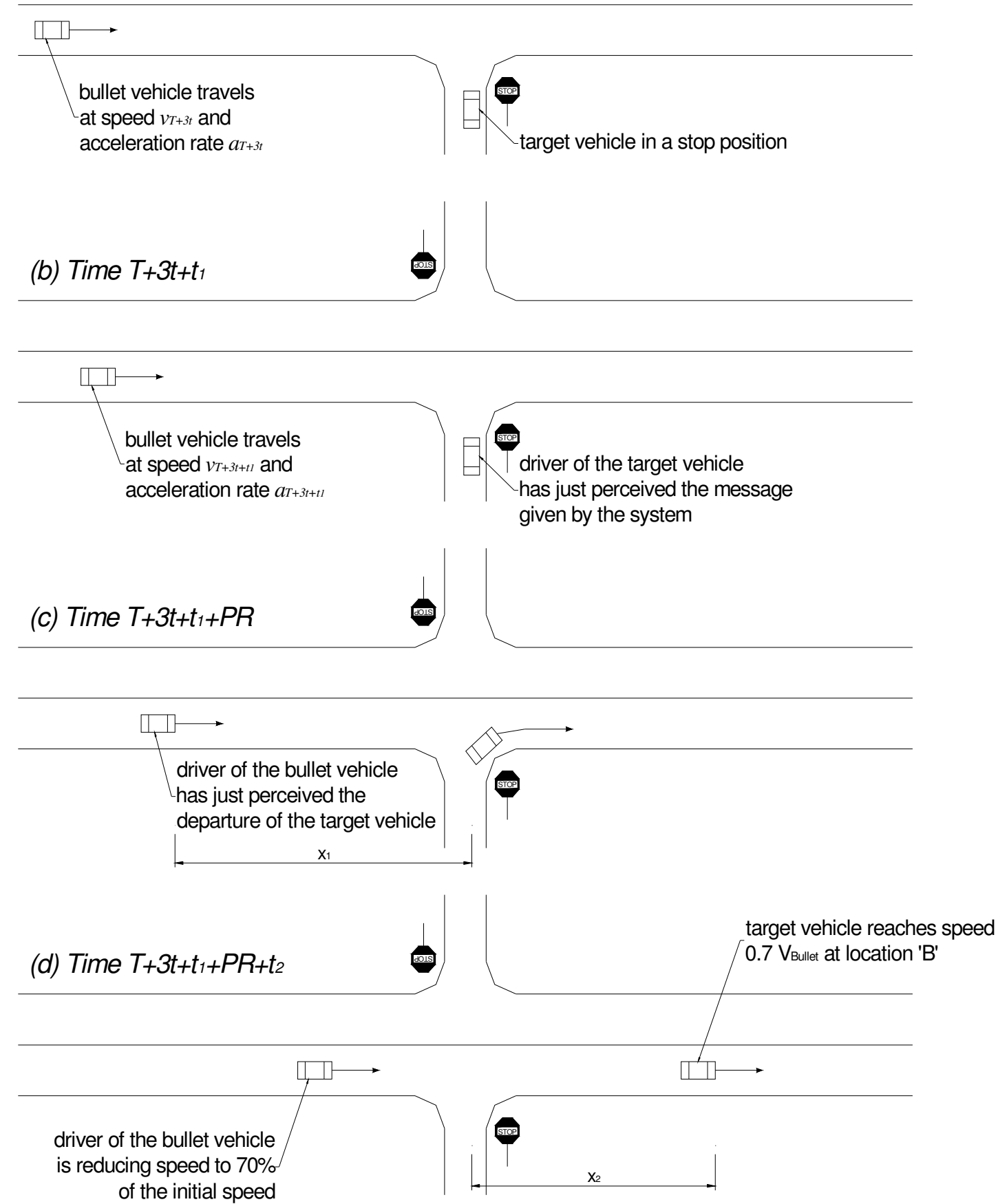

Figure 4.8 conflict when bullet and target vehicles are traveling on the same lane 


$$
\begin{aligned}
& v_{T+3 t}=v_{T}+3 t a_{T}+\frac{9}{2} r t^{2}=\frac{\frac{11}{6} d v_{1}-\frac{7}{6} d v_{2}+\frac{1}{3} d v_{3}}{t}+3 t\left(\frac{-2 d v_{1}+3 d v_{2}-d v_{3}}{t^{2}}\right) \\
& +\frac{9}{2} t^{2}\left(\frac{d v_{1}-2 d v_{2}+d v_{3}}{t^{3}}\right)=\frac{\frac{1}{3} d v_{1}-\frac{7}{6} d v_{2}+\frac{11}{6} d v_{3}}{t}
\end{aligned}
$$

2. At time $T+3 t+t_{1}+P R$ : the target vehicle has already started the departure movement while the driver of the bullet vehicle has just perceived the departure of the target vehicle and just starting to reduce its speed accordingly. The perception-reaction $(P R)$ time required for the driver of the bullet vehicle to perceive the departure of the target vehicle is selected to be 2.5 seconds as per the recommendations given by design guides and earlier research (AASHTO 2001; Fambro et al. 1997; Johansson and Rumar 1971; Normann 1953). The acceleration and speed at time $T+3 t+t_{1}+P R$ as well as the distance traversed from time $T+3 t$ to time $T+3 t+t_{1}+P R$ can all be computed from the following equations, respectively:

$$
a_{T+3 t+t_{1}+P R}=a_{T+3 t}+r\left(t_{1}+2.5\right)=\frac{d v_{1}-3 d v_{2}+2 d v_{3}}{t^{2}}+\left(t_{1}+2.5\right)\left(\frac{d v_{1}-2 d v_{2}+d v_{3}}{t^{3}}\right)[4
$$




$$
\begin{aligned}
& v_{T+3 t+t_{1}+P R}=v_{T+3 t}+\left(t_{1}+2.5\right) a_{T+3 t}+\frac{1}{2}\left(t_{1}+2.5\right)^{2} r= \\
& \frac{\frac{1}{3} d v_{1}-\frac{7}{6} d v_{2}+\frac{11}{6} d v_{3}}{t}+\left(t_{1}+2.5\right)\left(\frac{d v_{1}-3 d v_{2}+2 d v_{3}}{t^{2}}\right)+\frac{1}{2}\left(t_{1}+2.5\right)^{2}\left(\frac{d v_{1}-2 d v_{2}+d v_{3}}{t^{3}}\right) \\
& d v_{5}=\left(t_{1}+2.5\right) v_{T+3 t}+\frac{1}{2}\left(t_{1}+2.5\right)^{2} a_{T+3 t}+\frac{1}{6}\left(t_{1}+2.5\right)^{3} r=\frac{\left(t_{1}+2.5\right)}{t}\left(\frac{1}{3} d v_{1}-\frac{7}{6} d v_{2}+\frac{11}{6} d v_{3}\right) \\
& +\frac{\left(t_{1}+2.5\right)^{2}}{2 t^{2}}\left(d v_{1}-3 d v_{2}+2 d v_{3}\right)+\frac{\left(t_{1}+2.5\right)^{3}}{6 t^{3}}\left(d v_{1}-2 d v_{2}+d v_{3}\right)
\end{aligned}
$$

3. At time $T+3 t+t_{1}+t_{2}$ : the target vehicle has just finished its acceleration to $70 \%$ of the speed of the bullet vehicle (measured at time $T+3 t+t_{1}+P R$ ) at point ' $\mathrm{B}$ ' that is located at distance $x_{2}$ from the intersection. The time $t_{2}$ required for the target vehicle to accelerate to $70 \%$ of the speed of the bullet vehicle can be computed from the following equation (where $v_{T+3 t+t_{1}+P R}$ is calculated from Equation 4.36; $v_{e}$ is pre-loaded to the algorithm from the mechanical characteristics of the equipped target vehicle; $a_{d}$ is calculated from Equation 4.27 ; and $t_{2}$ is the only unknown variable):

$$
0.7 v_{T+3 t+t_{1}+P R}=a_{d} v_{e}\left(1-e^{-\frac{a_{d}}{v_{e}} t_{2}}\right)
$$


4. The distance $x_{5}$ required for the target vehicle to accelerate to $70 \%$ of the speed of the bullet vehicle (measured at time $T+3 t+P R$ ) can be computed from the following equation (where vehicle starts from stop):

$$
x_{5}=a_{d} v_{e} t_{2}-\frac{v_{e}^{2}}{a_{d}}\left(1-e^{-\frac{a_{d}}{v_{e}}}\right)
$$

5. The distance $x_{2}$, along the path of the bullet vehicle, between the intersection and point ' $\mathrm{B}$ ' can be computed from the following equation:

$$
x_{2}=x_{5}-w_{f}
$$

6. The time required for the bullet vehicle to reach point ' $\mathrm{B}$ ', $t_{\text {bullet, }}$, is the sum of the overall time required to travel a distance equals to the sum of $x_{1}$ and $x_{2}$, and it can be summarised as follows:

a. The time required to decelerate from speed $v_{T+3 t+t_{1}+P R}$ to $70 \%$ of that speed using a comfortable deceleration rate of $3.4 \mathrm{~m} / \mathrm{s}^{2}$ as suggested by design guides and earlier research (AASHTO 2001; Fambro et al. 1997), which can be computed from the following equation:

$$
t_{\text {bullet } 1}=\frac{0.3 v_{T+3 t+t_{1}+P R}}{3.4}
$$


b. The time required to travel on a constant speed $\left(0.7 v_{T+3 t+t_{1}+P R}\right)$ to reach the conflict point ' $\mathrm{B}$ ', which can be computed from the following equation:

$$
t_{\text {bullet } 2}=\frac{x_{3}}{0.7 v_{T+3 t+t_{1}+P R}}
$$

7. The distance $x_{3}$ where the bullet vehicle travels on a constant speed $\left(0.7 v_{T+3 t+t_{1}+P R}\right)$ can be computed from the following equation:

$$
x_{3}=x_{1}+x_{2}-x_{4}
$$

where

a. $x_{l}$ is calculated from the following equation (where $d_{f}$ is calculated from Equation 4.24 and $d v_{5}$ is calculated from Equation 4.37):

$$
x_{1}=d_{f}-d v_{5}
$$

b. $x_{2}$ is calculated from Equation 4.40

C. $x_{4}$ is the distance traveled while decelerating from $v_{T+3 t+t_{1}+P R}$ to $\left(0.7 v_{T+3 t+t_{1}+P R}\right)$, which can be calculated from the following Equation: 


$$
x_{4}=t_{\text {bullet }} v_{T+3 t+t_{1}+P R}-\frac{1}{2}(3.4) t_{\text {bullet } 1}^{2}=t_{\text {bullet } 1} v_{T+3 t+t_{1}+P R}-1.7 t_{\text {bullet } 1}^{2}
$$

8. Finally, the times required for both the target and bullet vehicles to reach the conflict point ' $\mathrm{B}$ ', from time $T+3 t$, are compared together as follows:

a. The total time required for the target vehicle to reach point ' $\mathrm{B}$ ' is calculated from the following equation (where $t_{1}$ is computed from the calibrated statistical model that will be discussed in Chapter 5 ; and $t_{2}$ is computed from Equation 4.38):

$$
t_{t \arg e t}=t_{1}+t_{2}
$$

b. The total time required for the bullet vehicle to reach point ' $\mathrm{B}$ ' is calculated from the following equation (where $P R$ is typically 2.5 seconds as given by design guides; $t_{\text {bullet }}$ is as computed from Equation 4.41; and $t_{\text {bullet2 }}$ is as computed from Equation 4.42):

$$
t_{\text {bullet }}=t_{1}+P R+t_{\text {bullet } 1}+t_{\text {bullet } 2}
$$

\subsection{Decision-Making Process}

The final step of the algorithm is to compare the time required for the bullet 
vehicle to reach the intersection, $t_{\text {bullet, }}$ as computed from Equation 4.25 or Equation 4.47 , with the time required for the target vehicle to depart the intersection, $t_{\text {target }}$, as computed from Equation 4.29 or Equation 4.46, using the following criterion:

$$
t_{\text {bullet }}>t_{\text {target }}
$$

If the above criterion is met for all detected vehicles, a 'safe' message is displayed to the driver; otherwise, the 'not safe' message continues to be displayed. 


\section{CHAPTER 5: DEVELOPMENT AND VALIDATION OF STATISTICAL MODELS}

This chapter describes the experimental procedures used to calibrate and validate the statistical models used to estimate the perception-reaction time for the driver of the equipped target vehicle, $t_{1}$, as well as the correction factor selected by the driver, $C_{d}$, for the maximum acceleration rate provided by the mechanical characteristics of the turning target vehicle. The chapter covers the methodology used to design the experiments, sample selection, the design of the simulation scenarios used in the experiments, data collection, data manipulation, the methods used to calibrate the final statistical models, and the methods used to validate those models.

\subsection{Experimental Design}

The objectives of the experiments can be summarised as follows (Dabbour et al. 2008):

1. to estimate the perception-reaction time for different driver groups when making a left-turn movement from a major road into a minor road (as was discussed in Chapter 3);

2. to estimate the perception-reaction time for different driver groups when departing a minor road into a major road on a typical semi-controlled 
intersection, either by turning left, turning right or passing through (as was discussed in Chapter 4); and

3. to estimate the correction factor selected by a driver, $C_{d}$, for the maximum acceleration rate provided by the mechanical characteristics of the turning target vehicle as used by different drivers in each of the above situations.

To achieve the above objectives, a set of simulation scenarios have been designed to simulate different driving situations shown above. The simulation scenarios are used with STISIM driving simulator (STISIM 2009) to simulate different real-life driving situations. The procedures of the experiment were to select a random sample of licensed drivers that is proportionally representative to licensed drivers in Ontario and Canada in terms of age and gender.

Every subject of the sample was asked to drive all different scenarios while measuring his/her response times and acceleration rates at different situations in each scenario. The collected data was used to develop regression models that predict the required dependent variables using the selected predictors so that the perception-reaction time and acceleration rate can be estimated for any other driver, who was not part of the sample used to develop the regression models, based on his/her known predictors (e.g., age and gender). 


\subsection{Simulation Scenarios}

To address the above objectives, a set of simulation scenarios have been developed. The set of scenarios comprises two groups of scenarios:

1. The first group of scenarios simulates left turn movement from a major road into a minor road through either a non-signalised intersection or a signalised intersection during either the amber or the permitted green phase; and

2. The second group of scenarios simulates the departure from a minor road into a major road in a semi-controlled intersection (by turning left, turning right or passing through).

To control for the variations in vehicle's mechanical performance, each group of the above scenarios comprises three scenarios that are almost identical where the only variation is the maximum acceleration rate that could be provided by the vehicle's mechanical characteristics as follows:

- The first scenario simulates a passenger car with a maximum rate of acceleration of $1.5 \mathrm{~m} / \mathrm{s}^{2}$, which represents a typical rate of acceleration for an older model passenger car with fair engine condition;

- The second scenario simulates a passenger car with a maximum rate of acceleration of $3.0 \mathrm{~m} / \mathrm{s}^{2}$, which represents a typical rate of acceleration for an average passenger car with good engine condition; and 
- The third scenario simulates a passenger car with a maximum rate of acceleration of $4.0 \mathrm{~m} / \mathrm{s}^{2}$, which represents a typical rate of acceleration for a top-of-the-line passenger car with excellent engine condition.

To control for the variations in road geometry that might affect the experiment, each scenario was designed to include 20 intersections that represent different geometric features. The geometric features that may have significant effect on the experiments are:

- The class of the highway (rural vs. urban);

- The number of lanes;

- The posted speed;

- The existence of a median (divided vs. undivided); and

- The traffic control device deployed (signalised vs. non-signalised).

Based on the above features, six intersections in the scenarios were signalised in urban settings, four were non-signalised in urban settings, and ten intersections were non-signalized in rural settings. Furthermore, the following road classes were included in the scenarios:

- Undivided two-lane rural road;

- Undivided four-lane urban arterial;

- Divided four-lane urban arterial; and

- Divided six-lane urban arterial. 
Typically, an STISIM scenario is defined by two files as follows:

1. a configuration file that defines vehicle characteristics, $1 / O$ configurations, and view settings; and

2. an events file that defines the road geometry, traffic characteristics as well as all different events that are faced by the subject during the experiment.

Typical scenarios from the first and the second groups are shown in Appendix "A" and Appendix "B", respectively. The algorithm for the proposed intersection collision warning system was encoded into all the scenarios so that at each intersection the subject would hear a buzz sound indicating that it was not safe to depart the intersection. Subjects were instructed to depart every intersection as soon as the buzz signal stops for that intersection.

The data collected at each intersection include the perception-reaction time for the subject from the time the buzz signal stops to the time when the subject starts to engage the throttle to complete the required movement. The data collected also included the average acceleration rate for the subject during the departure movement.

\subsection{Sample Selection}

To ensure that the results are as realistic as possible, the stratified sample size was selected so that it proportionally represents the total population of licensed 
drivers in Ontario and Canada in terms of gender and age groups. This method of proportional representation of the population was found to be a powerful approach in sample selection (Dorofeev and Grant, 2006; Moser and Kalton 1971). In 2003, the total number of licensed drivers in Ontario was found to be $8,541,555$ licensed drivers (MTO 2003), from which $53 \%$ were males and $47 \%$ were females. The total number of licensed drivers in Canada for the same year (2003) was found to be $21,427,287$ licensed drivers (Transport Canada 2003) with the male-female proportional is the same as in Ontario ( $53 \%$ males and $47 \%$ females). Driver populations by age group for both Ontario and Canada are shown in Table 5.1. Figure 5.1 shows comparison for the proportion of total driver population for each age group in both Ontario and Canada.

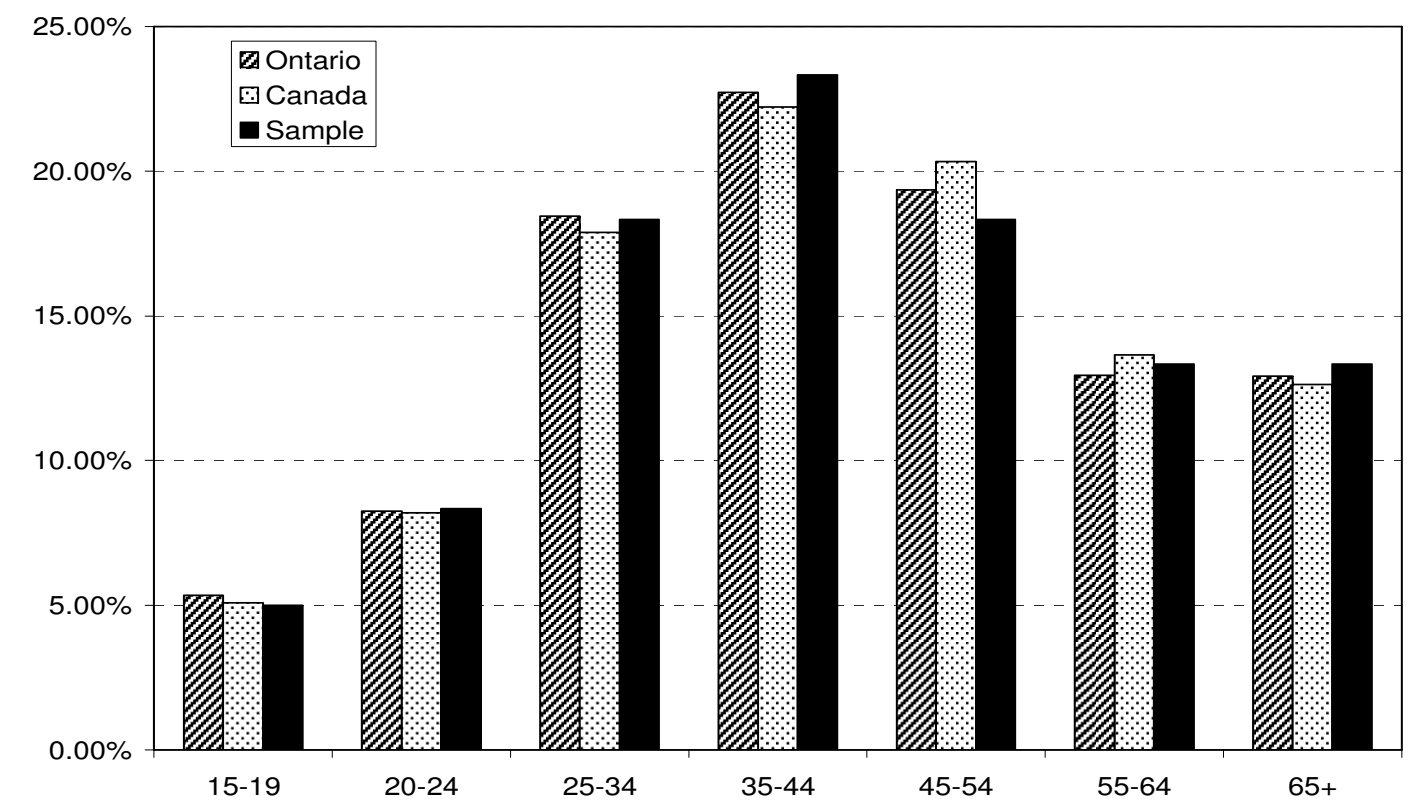

Figure 5.1 Driver populations in Ontario and Canada (2003) compared to sample 
Table 5.1 Licensed drivers in Ontario and Canada by age group (2003)

\begin{tabular}{|l|c|c|c|c|}
\hline \multirow{2}{*}{ Age } & \multicolumn{2}{|c|}{ Ontario } & \multicolumn{2}{c|}{ Canada } \\
\cline { 2 - 5 } Group & Number of Licensed & & Number of Licensed & \\
& Drivers & Percentage & Drivers & Percentage \\
\hline $15-19$ & 457,049 & $5.35 \%$ & $1,087,986$ & $5.08 \%$ \\
$20-24$ & 704,720 & $8.25 \%$ & $1,754,394$ & $8.19 \%$ \\
$25-34$ & $1,575,345$ & $18.44 \%$ & $3,833,556$ & $17.89 \%$ \\
$35-44$ & $1,940,896$ & $22.72 \%$ & $4,760,515$ & $22.22 \%$ \\
$45-54$ & $1,653,604$ & $19.36 \%$ & $4,358,434$ & $20.34 \%$ \\
$55-64$ & $1,105,726$ & $12.95 \%$ & $2,924,581$ & $13.65 \%$ \\
$65+$ & $1,104,215$ & $12.93 \%$ & $2,707,821$ & $12.64 \%$ \\
\hline Total & $\mathbf{8 , 5 4 1 , 5 5 5}$ & $\mathbf{1 0 0 . 0 0 \%}$ & $\mathbf{2 1 , 4 2 7 , 2 8 7}$ & $\mathbf{1 0 0 . 0 0 \%}$ \\
\hline
\end{tabular}

Both Table 5.1 and Figure 5.1 show near similarity between Ontario and Canada in the proportion of total driver population for each age group. Based on that, the stratified sample structure that represents the population of drivers in Ontario and Canada may include male proportion within the range (50-53\%) and proportions of age groups as close as possible to those shown in Table 5.1. A practical sample size that satisfies the above requirements is to have 60 drivers randomly selected from the population with their gender and age proportions as shown in Table 5.2. As shown in Figure 5.1, the proportions of age groups in the sample are reasonably close to those in both Ontario and Canada. 
Table 5.2 Sample size for the experiment

\begin{tabular}{|l|c|c|c|}
\hline Age Group & Male & Female & Percentage \\
\hline $15-19$ & 2 & 1 & $5.00 \%$ \\
$25-24$ & 3 & 2 & $8.33 \%$ \\
$35-44$ & 6 & 5 & $18.33 \%$ \\
$45-54$ & 7 & 7 & $23.33 \%$ \\
$55-64$ & 4 & 4 & $13.33 \%$ \\
$65+$ & 4 & 4 & $13.33 \%$ \\
\hline Total & $\mathbf{3 2}$ & $\mathbf{2 8}$ & $\mathbf{1 0 0 . 0 0 \%}$ \\
\hline
\end{tabular}

It should be noted that the sample size selected may not be fully representative of the population in terms of driving behaviour, especially with the lack of statistical parameters of the population with respect to the phenomena described by the statistical models, namely the perception-reaction time, $t_{1}$, and correction factor for the maximum acceleration rate provided by the equipped vehicle, $C_{d}$, for the case when a collision warning system is installed. However, it is impractical to substantially increase the sample size, especially with the lack of the statistical parameters of the population that leads to uncertainty in determining the required sample size that produces an acceptable margin of error between the sample and the population. 
The sample size selected is within the same range of the sample sizes selected by similar previous driver simulation studies (Staplin 1995; Alexander et al. 2002; Yan et al. 2007; Yan et al 2008). It should be noted that the regression models developed from the sample size used are only applicable at the early stage of implementing the system and they should be later replaced by more-realistic self-learning artificial neural network models, as will be explained in subsequent sections in this chapter.

\subsection{Experimental Procedures}

The experiments have taken place in the Road Safety Laboratory located at the Civil Engineering Department, Ryerson University using STISIM driving simulator. A total of 60 subjects have been invited to drive the following simulation scenarios:

- A set of scenarios to simulate left-turn movement from a major road (as was discussed in Chapter 3); and

- Another set of scenarios to simulate vehicle departure from a minor road in a semi-controlled intersection (as was discussed in Chapter 4).

The purpose of the experiment was explained to every subject before starting the simulations, along with a brief description for the simulation scenarios and instructions for what was expected from the subject. In addition to the verbal briefing, an instruction sheet was also given to the subject that explains the process of the 
simulation scenarios and gives him/her all the required instructions to drive the scenarios.

The data collected from the subjects before the start of the experiments include subject's age, gender, class of driver's license, and number of years of driving experience. The data collected by the STISIM software at every intersection during the experimental work include the perception-reaction time of the subject from the time the buzz signal stops to the time when the subject starts to engage the throttle to complete the required movement. The data collected also include the average acceleration rate for the subject during the departure movement. All the data have been aggregated for manipulation in order to develop the regression models.

\subsection{Regression Models}

A total of 3600 observations were used to calibrate the regression models for leftturning drivers. From the observations taken, two of the subjects failed to depart the intersection within the time gap provided by the simulation, which resulted in two left turn collisions (in the simulation).

For the situation of departing a minor road, 2160 observations were used to calibrate the regression models, and from those observations, one subject failed to depart the intersection within the time gap provided by the simulation, which also resulted in another angle collision (in the simulation). All the three simulation collisions may be interpreted as a result if drivers' unfamiliarity with the system so that the drivers took excessive time to verify the 'safe' message given by the system 
and their reaction was so slow that they overestimated the time remaining of the gaps provided by the system after they had consumed noticeable part of those gaps verifying the message given by the system.

Four different regression models have been developed using the data collected from the experiments, which are listed below:

- The first regression model estimates driver's perception-reaction time (PRT) for left turn situation when the driver attempts to make a left turn from a major road into a minor road in either a non-signalised intersection or a signalised intersection during the permitted green time;

- The second regression model estimates driver's correction factor for the acceleration rate (CAR) for left turn situations;

- The third regression model estimates driver's perception-reaction time (PRT) when departing a minor road in a semi-controlled intersection; and

- The fourth regression model estimates driver's correction factor for the acceleration rate (CAR) when departing a minor road in a semi-controlled intersection.

\subsubsection{Left Turn Situation}

Three scenarios were developed to simulate left turn movement. All the three scenarios were almost identical where the only variation was the maximum acceleration rate provided by vehicle's mechanical characteristics, as was previously discussed. A total of 3600 observations were used to calibrate the following 
regression models for perception-reaction time, $t_{1}$, and correction factor for acceleration rate, $c_{d}$, respectively:

$$
\begin{gathered}
t_{1}=0.2466+0.0241 A G E+0.1353 G E N D E R \\
c_{d}=0.95164-0.00228 A G E-0.01976 G E N D E R-0.00517 d_{f}+0.02325 v_{f}
\end{gathered}
$$

where $A G E$ is the age of the driver (in years), GENDER is a dummy variable that represents driver gender ( 0 for male and 1 for female), $d_{f}$ is the distance (in meters) to the nearest detected approaching vehicle and $v_{f}$ is the speed of the nearest approaching vehicle (in meter per second).

The relevant statistics for both models are shown in Table 5.3 and Table 5.4, respectively. The coefficients of all independent variables are significantly different from zero at the $95 \%$ confidence level.

Table 5.3 Summary of statistics for estimating $t_{1}$ (left turn)

\begin{tabular}{lll}
\hline Independent Variable & Coefficient & t-stat \\
\hline Intercept & 0.2466 & 31.887 \\
$A G E$ & 0.0241 & 156.889 \\
GENDER & 0.1353 & 26.613 \\
\hline
\end{tabular}

Note: Coefficient of determination, $R^{2}=0.87$; significance of $F$ statistic $=0.000$

Table 5.4 Summary of statistics for estimating $c_{d}$ (left turn)

\begin{tabular}{lcl}
\hline Independent Variable & Coefficient & t-stat \\
\hline Intercept & 0.95164 & 170.6739 \\
$A G E$ & -0.00280 & -151.586 \\
$G E N D E R$ & -0.01976 & -39.755 \\
$d_{f}$ & -0.00517 & -157.888 \\
$v_{2}$ & 0.02325 & 104.5611 \\
\hline Note: Coefficient of determination, $R^{2}=0.96 ;$ significance of $F$ statistic $=0.000$
\end{tabular}


There are logical explanations for the parameters shown in both regression models. For the PRT model, the positive sign associated with the age indicates that perception-reaction time increases with the age as older drivers usually have slower cognitive response than younger drivers, which is consistent with what was found in earlier research (American Automobile Association 1958; Lerner et al. 1995; Alexander et al. 2002; Yan et al. 2007). The positive sign for the parameter associated with the gender indicates that perception-reaction time for female drivers is longer than that for male drivers by approximately 0.13 seconds under the same conditions, which is also consistent with earlier research findings (Alexander et al. 2002; Yan et al. 2007).

For the CAR model, the negative sign for the parameter associated with the age indicates that older drivers accelerate slower than younger drivers due to the increased experience and reduced vision so that their driving behavior is less risky. This finding is also consistent with earlier research findings (Yan et al. 2007). The negative sign for the parameter associated with the gender indicates that female drivers accelerate slower than male drivers by approximately $1.86 \%$ under the same conditions. The negative sign for the parameter associated with the distance to the approaching vehicle indicates that drivers tend to accelerate faster when the approaching vehicles are closer so that they can avoid any imminent collision. Finally, the positive sign for the parameter associated with the speed of the approaching vehicle indicates that drivers tend to accelerate faster to avoid being hit by the approaching vehicles that are moving with higher speeds. The findings for the effect of the speed and proximity of the approaching vehicle are also consistent with 
the findings of earlier research that used both driving simulation and actual field data (Alexander et al. 2002; Davis and Swenson 2004; Yan et al. 2007).

\subsubsection{Semi-Controlled Intersections}

Similar to the left-turn scenarios, three other scenarios have also been developed to simulate the departure from a semi controlled intersection where a vehicle attempts to depart a controlled minor road into a non-controlled major road (by turning left, turning right or passing through). A total of 2160 observations were used to calibrate the following regression models for perception-reaction time, $t_{1}$, and correction factor for acceleration rate, $c_{d}$, respectively:

$$
\begin{aligned}
& t_{1}=0.3726+0.0278 A G E+0.1523 G E N D E R \\
& c_{d}=0.95745-0.00219 A G E-0.01860 G E N D E R-0.00471 d_{f}+0.02234 v_{2}
\end{aligned}
$$

All the variables are previously defined for the left turn models. The relevant statistics for both models are shown in Table 5.5 and Table 5.6, respectively. The coefficients of all independent variables are significantly different from zero at the 95\% confidence level. All parameters have logical explanations similar to those for left turn models. 
Table 5.5 Summary of statistics for estimating $t_{1}$ (departing a controlled minor road)

\begin{tabular}{lll}
\hline Independent Variable & Coefficient & t-stat \\
\hline Intercept & 0.3726 & 21.089 \\
$A G E$ & 0.0278 & 78.974 \\
GENDER & 0.1523 & 13.106 \\
\hline
\end{tabular}

Note: Coefficient of determination, $R^{2}=0.75$; significance of $F$ statistic $=0.000$

Table 5.6 Summary of statistics for estimating $c_{d}$ (departing a controlled minor road)

\begin{tabular}{lll}
\hline Independent Variable & Coefficient & t-stat \\
\hline Intercept & 0.95745 & 89.69364 \\
$G E N D E R$ & -0.01860 & -19.25965 \\
$A G E$ & -0.00219 & -75.06292 \\
$d_{f}$ & -0.00471 & -80.71803 \\
$v_{2}$ & 0.02234 & 50.04220 \\
\hline
\end{tabular}

Note: Coefficient of determination $R^{2}=0.91$ and significance of $F$ statistic $=0.000$

\subsection{Model Validation}

The regression models were validated by asking a sample of 6 licensed drivers, who were not included in the sample used to calibrate the models, to drive the same simulation scenarios on the STISIM driving simulator. The validation sample included 3 males and 3 females with different ages. The margin of error between the validation sample and the calibration sample can be calculated from the following equation:

$$
E=\frac{z_{\alpha / 2} \sigma}{\sqrt{n}}
$$

where:

$$
\begin{array}{ll}
n & =\text { sample size } \\
E & =\text { margin of error between calibration and validation samples }
\end{array}
$$




$$
\begin{array}{ll}
\sigma & =\text { standard error for the calibration sample } \\
z_{\alpha / 2} & =\text { critical value (typically } 1.96 \text { for } 95 \% \text { confidence level) }
\end{array}
$$

The margin of error between the calibration and validation samples is shown in Table 5.7, which is based on Equation 5.5 where the standard error values, associated with calibrating the models, have been applied. From Table 5.7, it is evident that the sample size selected for the validation results in a margin of error that is insignificant for all calibrated statistical models.

To validate the statistical models over all different ages, the ages of the drivers used to validate the models were different from the ages used in calibrating the models. A total of 360 observations were used to validate the models for left-turn movement, and a total of 216 observations were used to validate the models for departing a controlled minor road. The validation statistics for all the models are shown in Table 5.8, which include the following:

- The sum of validation errors, $S E_{v}$, which is the sum of the differences between the observed and the predicted values (either perception-reaction times or correction factor for acceleration rate). The sum of validation errors is computed by using the following formula:

$$
S E_{v}=\sum_{i=1}^{n_{v}} y_{i}-\hat{y}_{i}
$$


- The sum of squares of validation errors, $S S E_{v}$, which is the sum of the squared differences between the observed and the predicted values; and it can be computed by using the following formula:

$$
S S E_{v}=\sum_{i=1}^{n_{v}}\left(y_{i}-\hat{y}_{i}\right)^{2}
$$

- Mean squared error of validation, $M S E_{v}$, which is the average squared error for validation, or the sum of squares of errors divided by the number of validation observations:

$$
M S E_{v}=\frac{S S E_{v}}{n_{v}}
$$

- Root mean squared error of validation, $R M S E_{v}$, which is a measure of the average error of validation among all the validation observations, and is computed as the square root of the mean squared error of validation:

$$
R M S E v=\sqrt{M S E_{v}}=\sqrt{\frac{\sum_{i=1}^{n_{v}}\left(y_{i}-\hat{y}_{i}\right)^{2}}{n_{v}}}
$$

Table 5.7 Margin of error between calibration and validation samples

\begin{tabular}{cccc}
\hline \multicolumn{2}{c}{ Left-turn movement } & \multicolumn{2}{c}{ Departing controlled minor road } \\
$t_{l}$ & $c_{d}$ & $t_{l}$ & $c_{d}$ \\
\hline 0.1218 & 0.0119 & 0.2157 & 0.0179 \\
\hline
\end{tabular}


Table 5.8 Summary of validation statistics for all regression models

\begin{tabular}{lcccc}
\hline Validation Measure & \multicolumn{2}{c}{ Left-turn movement } & \multicolumn{2}{c}{ Departing controlled minor road } \\
& $t_{l}$ & $c_{d}$ & $t_{l}$ & $c_{d}$ \\
\hline$S E_{v}$ & 0.89 & 0.15 & 1.37 & 0.41 \\
$S S E_{v}$ & 11.2161 & 0.1966 & 12.4729 & 0.1298 \\
$M S E_{v}$ & 0.03116 & 0.00055 & 0.05774 & 0.00060 \\
$R M S E_{v}$ & 0.17651 & 0.02337 & 0.24030 & 0.02451 \\
$S_{e}$ (from calibration) & 0.15227 & 0.01489 & 0.26951 & 0.02240 \\
No. of observations & 360 & 360 & 216 & 216 \\
\hline
\end{tabular}

From Table 5.8, the sum of the validation errors is small for all the models, which indicates that the errors resulting from validating the models fluctuate around zero and do not accumulate to a negative or a positive trend. The mean squared validation error for all the models is insignificant. Furthermore, the root mean squared errors of validation, $R M S E_{v}$, is very small (less than 0.24 seconds in calculating $t_{1}$, and less than $2.24 \%$ in calculating the correction factor for acceleration rates). Also, the root mean squared errors, $R M S E_{v}$, were very close to the standard errors associated with model calibration, indicating that the models are stable and robust when being validated for predictors that were not used in calibrating the models.

\subsection{Artificial Neural Network Models}

The reliability of the prediction models can be improved by recording the actual perception-reaction times and acceleration rates for specific driver(s) on a specific vehicle and creating a database for every driver to be used to calibrate nonlinear prediction models using artificial neural networks that replace the original regression models. The developed artificial neural network (ANN) models have the potential to forecast perception-reaction times (PRT) and the driver's correction factors for 
acceleration rates (CAR) by recognizing patterns of the observed data collected from individual drivers.

ANN is capable of capturing such highly nonlinear phenomena and has been applied to various problems in the field of transportation engineering, where ANN has been used in driver identification based on voice signal (Wu and Ye 2009), analysis of vehicle accident frequency in freeways (Chang, 2005), injury severity in traffic collisions (Delen et al. 2006), vehicle collisions (Xie et al. 2007) and vehicle accident reconstruction (Xiao-yun et al. 2008).

Typically, ANN consists of multiple layers of many interconnected linear or nonlinear processing units operating in a parallel fashion. Each processing unit receives multiple inputs through weighted connections from neurons in the previous layer to which it is connected, performs appropriate computations, and transmits its output to other processing units as a network output using an assigned transfer function (Rumelhart et al. 1986). The nonlinear nature of neural networks makes them suitable to perform functional approximation, classification, and pattern recognition (Bishop 1995; Freeman and Skapura 1991). Three important steps must be considered in constructing a successful artificial neural network:

- network architecture;

$\circ$ training; and

$\circ$ testing.

The basic methodology for developing a successful ANN-based model is to teach a neural network the relationship between inputs and outputs. The basic aspects of 
network architecture consist of the number of hidden layers between the input and output layers (as shown in Figure 5.2), the number of processing units in each layer, the pattern of connectivity among the processing units, and the activation (transfer) function employed for each processing unit (Zhang et al. 1998). Upon successful completion of the training process, a well-trained neural network is not only capable of computing the expected output of any input set of data used in the training stage, but also should be able to predict with an acceptable accuracy the outcome of any unfamiliar set of input located within the range of the training data.

In this research, ANN-based feed-forward models (the most popular and widely used network paradigm in forecasting) are developed which can forecast and recognize patterns of perception reaction time and acceleration rate of an individual driver on a specific vehicle. The actual perception reaction times and acceleration rates for specific driver on a specific vehicle as well as the distance $\left(d_{f}\right)$ and the speed $\left(v_{2}\right)$ of the nearest detected approaching vehicle are used to create a database for every driver for training and validation of ANN models that can replace the original regression models by showing better predictive ability and reliability.

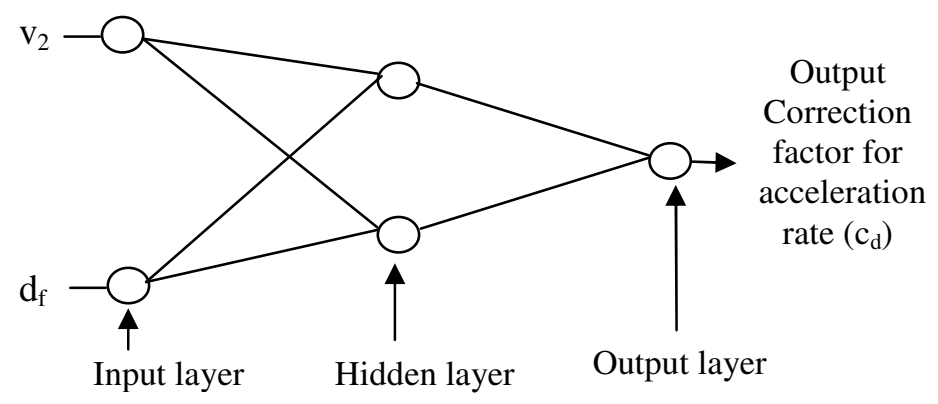

Figure 5.2 Typical multilayered feed-forward (2:2:1) ANN model 
As the ANN models are developed for each driver, other parameters, such as age and gender, were not included. Four different ANN models have been developed using MATLAB codes as follows:

1. The first model (ANN1) predicts the perception-reaction time (PRT) for a specific driver at left-turn situations. This model is based on a MATLAB toolbox that was specifically developed for artificial neural networks (Nogaard 2000).

2. The second model (ANN2) predicts the perception-reaction time (PRT) for the specific driver when departing a controlled minor road in a semi-controlled intersection. This model is also based on a MATLAB toolbox that was specifically developed for artificial neural networks (Nogaard 2000).

3. The third model (ANN3) predicts driver's correction factor for acceleration rate (CAR) related to a specific driver at left-turn situations. This model is based on MATLAB toolbox for feed-forward systems (The Mathworks 2009).

4. The fourth model (ANN4) predicts driver's correction factor for acceleration rate (CAR) related to the specific driver when departing a controlled minor road in a semi-controlled intersection. This model is also based on MATLAB toolbox for feed-forward systems (The Mathworks 2009).

The objective of ANN1 and ANN2 is to predict the value of perception reaction time (PRT) of an individual driver for a time in the future by using feed-forward ANN models with one-step-ahead prediction. In such prediction, a set of values (for PRT) from a number of periods, for example three successive periods $x_{t-2}, x_{t-1}$ and $x_{t}$ are 
used as the input vector of the network and the next value $x_{t+1}$ is used as the target value as illustrated in Figure 5.3. By moving along the time axis, a training data set is created that consists of many sets of input vector values with corresponding target values (Bishop, 1995). The one-step-ahead prediction is achieved by 'training' the ANN model using the actual dataset that can be represented as per the following equation (where $u(i)$ is the set of inputs, $y(i)$ is the corresponding outputs and $N$ is the number of inputs):

$$
Z^{i}=\{[u(i), y(i)] i=1, \ldots, N\}
$$

ANN3 and ANN4 are developed to predict the value of correction factor $\left(c_{d}\right)$ for acceleration rate $(\mathrm{CAR})$ of an individual driver using general feed-forward networks.

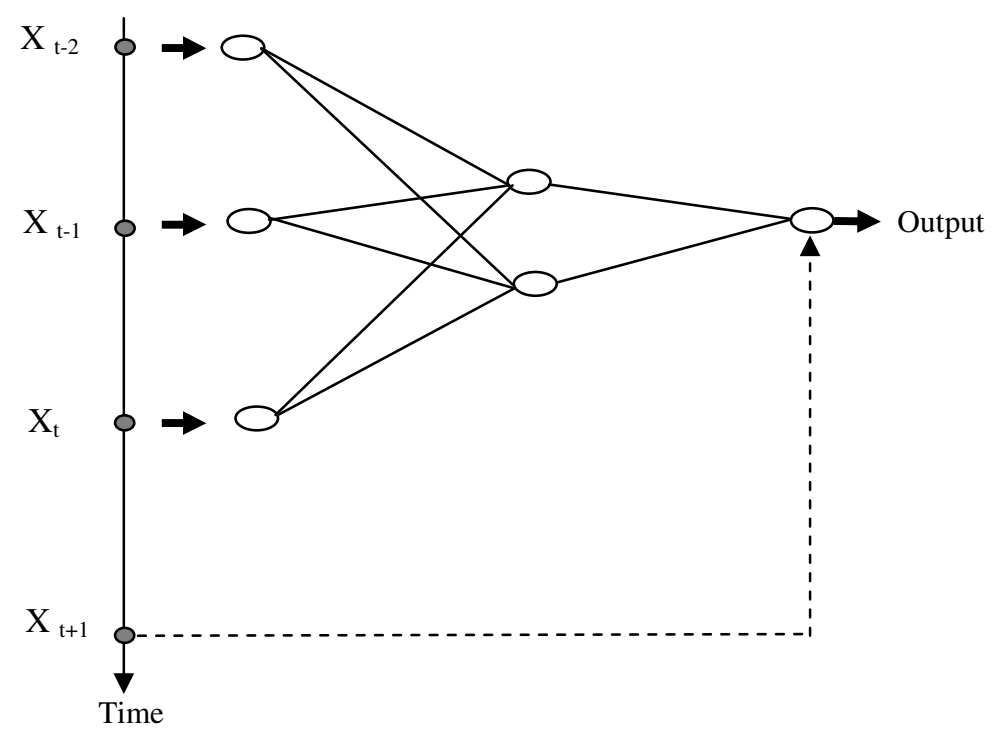

Figure 5.3 Feed-forward network with one-step-ahead prediction 
These models are developed with 2 variables in the input layer $\left(d_{f}\right.$ and $\left.v_{2}\right), 2$ neurons in a single hidden layer (obtained from network optimization) and a single output (correction factor, $c_{d}$, for acceleration rate) in the output layer. Typical architecture of such 2:2:1 ANN model is shown in Figure 5.2.

All four ANN models developed in MATLAB codes have been trained and validated by using the database created from experimental observations of 60 drivers used to develop the original linear regression models.

There were 60 observations from every driver for left-turn situations, and 36 observations from every driver for the situation when departing a controlled minor road. The idea was to use part of the dataset for every driver to 'train' the ANN model, and then validate the model by comparing the predicted dependent variables to their corresponding observed actual values in the remaining part of the original dataset.

To evaluate the performance of the developed ANN models in comparison to the original linear models, the values for the root-mean-squared error (RMSE) for all drivers in all situations are shown in Table 5.9. In addition, Figure 5.4 to Figure 5.7, consecutively, show the predicted values for PRT and correction factor for CAR compared with their observed values for a typical driver.

From the table and the figures, the developed ANN models were found to perform very well in predicting perception-reaction time (PRT) and correction factor for acceleration rate (CAR) for every driver as predicted values were found to be close to the observed values. This demonstrates that the developed ANN models are robust and yield reasonable values with acceptable errors. However, given the relatively 
small number of observations used in calibrating the ANN models, RMSE values for ANN1 and ANN2 (perception-reaction time) were found to exceed those for the initial linear regression models, which indicates that more observations are needed to yield RMSE values that are less than those for the original regression models (Tables 5.8 and 5.9).

The performance of ANN3 and ANN4 models (for acceleration rate's correction factor) seems to be better than the original regression models given the lower RMSE values. However, the neural network model should not replace the original regression model until the size of the dataset, $N$, produces values for the root-mean-squarederror (RMSE) that are less than those for the original linear regression models, which is the criterion used to fully migrate from the original initial regression models to the more-sophisticated artificial neural network (ANN) models.

Table 5.9 Root-mean-squared errors for all sample drivers (ANN models)

\begin{tabular}{ccccc}
\hline RMSE & \multicolumn{2}{c}{ Perception-reaction Time } & Correction Factor for Acceleration Rate \\
& & ANN2 & ANN4 \\
& ANN1 & Departing minor & ANN3 & Departing minor \\
& Left-turn & road & Left-turn & road \\
\hline Minimum & 0.06 & 0.13 & 0.010 & 0.010 \\
Maximum & 0.63 & 0.64 & 0.022 & 0.051 \\
Mean & 0.19 & 0.37 & 0.015 & 0.021 \\
\hline
\end{tabular}




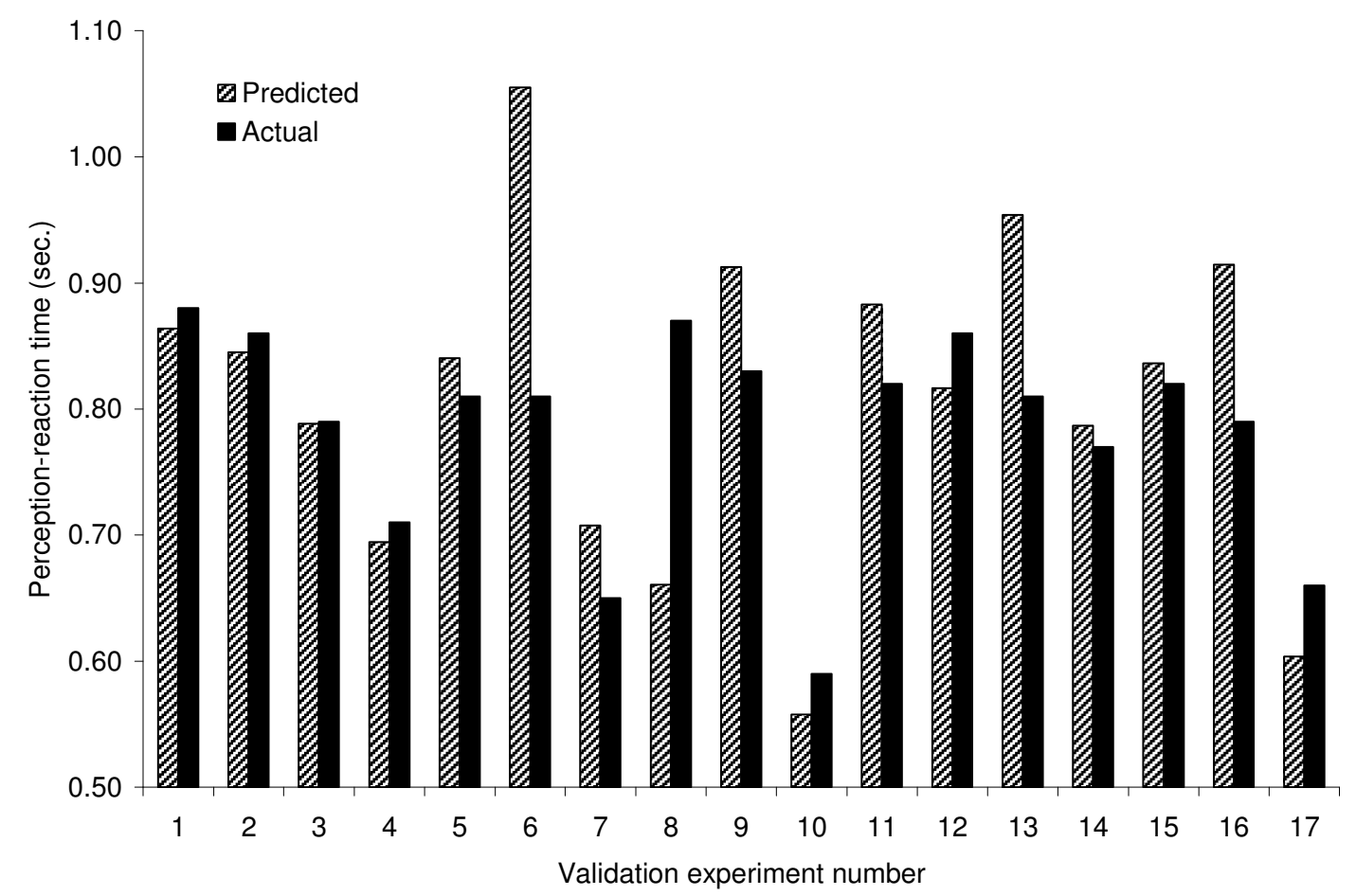

Figure 5.4 ANN prediction for the perception-reaction time (left turn situation)

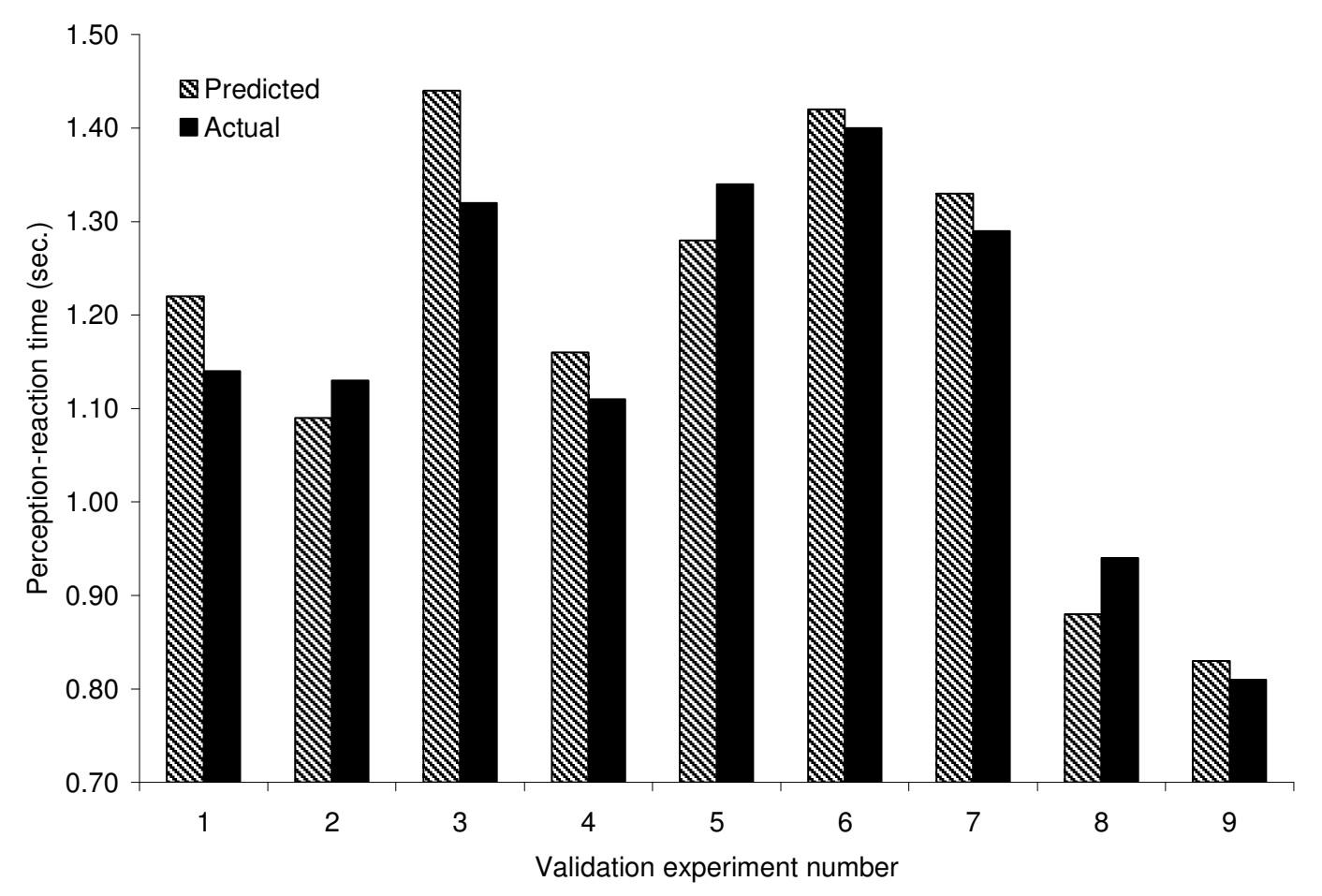

Figure 5.5 ANN prediction for the perception-reaction time (departing minor road) 


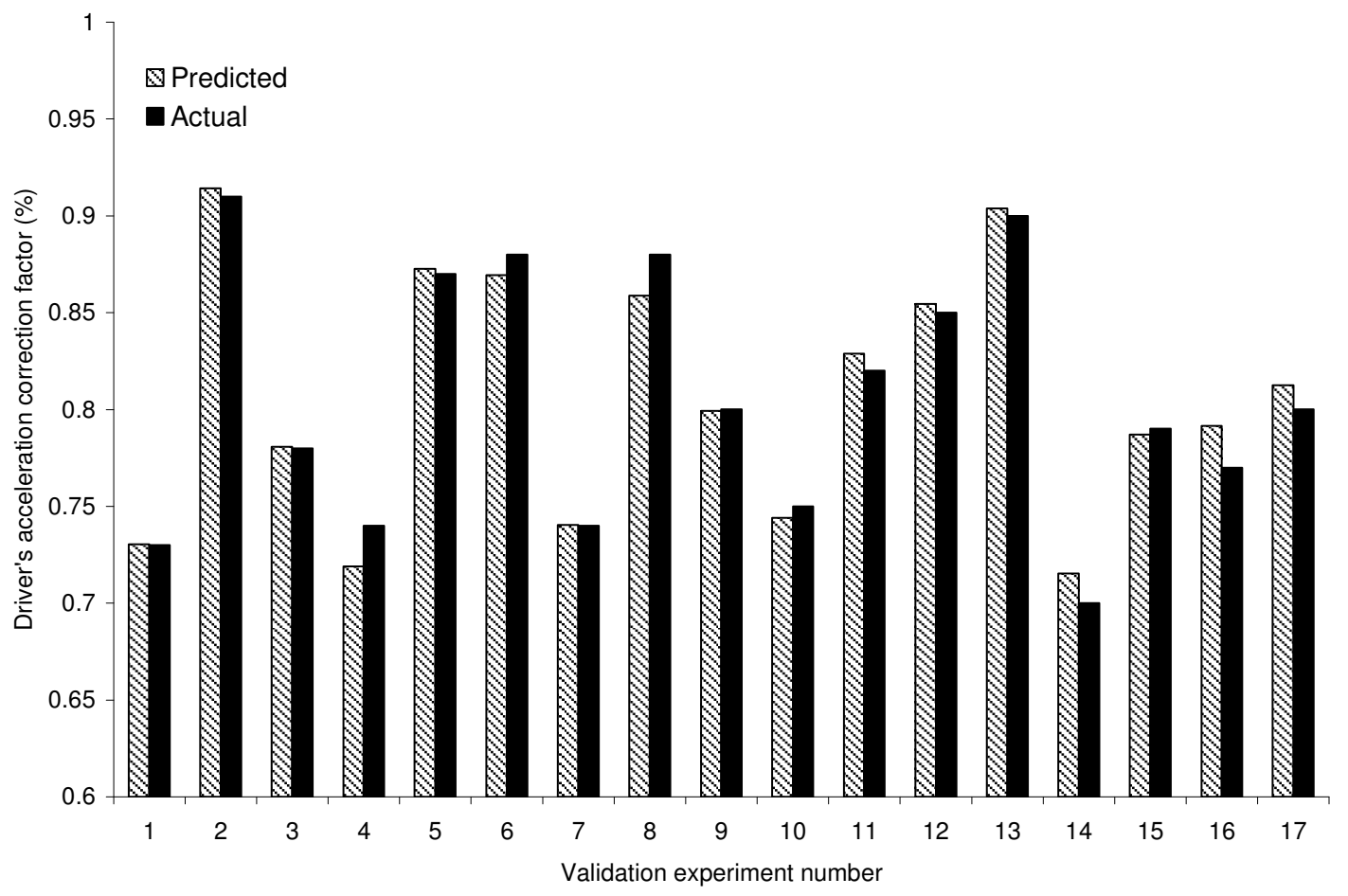

Figure 5.6 ANN prediction for diver's acceleration (left turn situation)

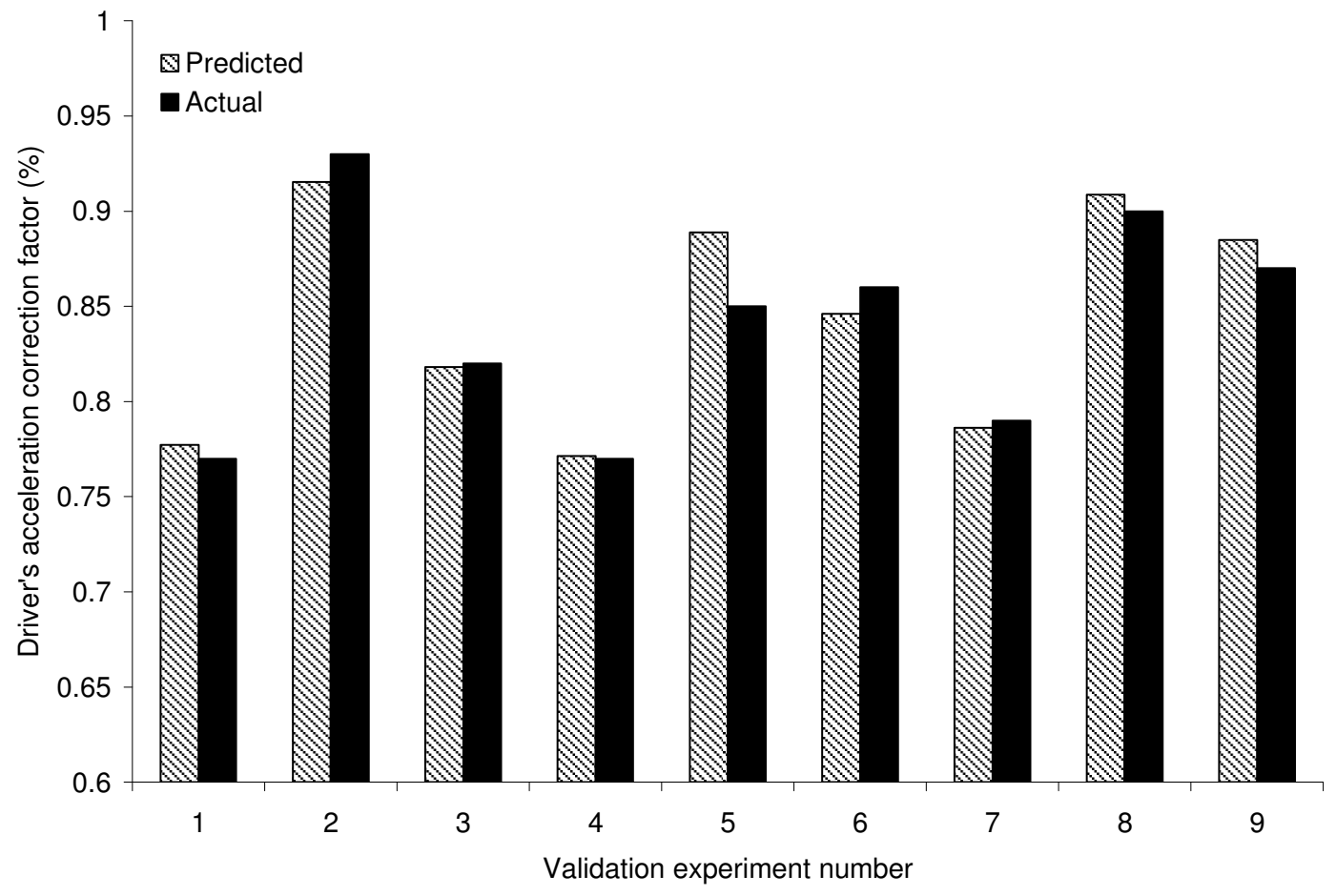

Figure 5.7 ANN prediction for diver's acceleration (departing minor road) 


\section{CHAPTER 6: SYSTEM VALIDATION AND SENSITIVITY ANALYSIS}

To validate the algorithms used in the proposed system, two MATLAB codes were created where the first code, TWSC_VALID, estimates positions, speeds, and acceleration rates of all vehicles at different time instances using the laws of dynamic motion; while the second code, TWSC_SIM, estimates them by applying the principles of trigonometric functions on the sensor readings, including range distances and azimuth angles, given at four consecutive time instances $(T ; T+t ; T+2 t$; $T+3 t)$ as was discussed in Chapter 3 and Chapter 4 .

This chapter gives details on the procedures followed to create both MATLAB codes and the methodology used to validate the system using both codes and also the methodology used to utilize both codes to examine different factors that may impact the accuracy of the system and how to assess the impact of those factors to optimize the effectiveness of the system.

It should be noted that there is similarity between left-turn situation and TWSC situation from analytical point of view with the exception that TWSC situation needs more analysis for the two additional conflict cases discussed in section 4.3 and shown in Figure 4.2(a) and Figure 4.2(c); and therefore, all the results obtained from the TWSC analysis, discussed in this chapter, are also applicable to left-turn situation except those results related to conflict cases that are not applicable to left-turn situation (i.e., when the paths of the target 
and bullet vehicles are parallel to each other and when they both travel on the same lane at the same direction).

\subsection{Validation Code}

The first MATLAB code, TWSC_VALID, is shown in Appendix ' $C$ '. It is based on the laws of dynamic motion where the user would input the initial conditions of every approaching vehicle (including its initial distance to the intersection, $l$, its initial speed, $v_{T}$, initial acceleration, $a_{T}$, and the rate of change in acceleration, $r$ ). The user should also input the specifications related to the detection sensors, including installation angle, the time interval used, the opening angle of the detection sensor, and the reflective point of the vehicle detected by the sensor (either the near edge of the vehicle, the centre of the vehicle or the far edge of the vehicle).

Driver specific information should also be input, including driver's age, gender and the turning signal activated. The mechanical characteristics of the host target vehicle should also be input, including its length, the maximum acceleration rate provided by its engine and the equilibrium speed where the acceleration decreases to zero (the crawl speed). Finally, the user should also input all the information related to geometric configuration of the roadway, including the number of lanes, lane width, median width, and setback distance.

The code estimates the displacement of every approaching vehicle at any time instance by applying the following equation (where $c_{n}$ is the displacement at time instance $(T+n t), n$ is the time interval number, and $t$ is the time interval length): 


$$
c_{n}=v_{T}(n t)+\frac{1}{2} a_{T}(n t)^{2}+\frac{1}{6} r(n t)^{3}
$$

The code computes the range at any time instance by applying the following equation (where the offset distance, $w_{f}$, is computed by the system based on the location of the approaching vehicle as defined by the user):

$$
d_{n}=\sqrt{w_{f}^{2}+\left(l-c_{n}\right)^{2}}
$$

The code also computes the azimuth angle (measured to the longitudinal axis of the detection sensor) at any time instance. Computing the azimuth angle depends on whether the bullet vehicle is approaching from the left or from the right. For a vehicle approaching from the left, the azimuth angle is computed by applying the following equation (where $\theta_{n}$ is the azimuth angle, $\alpha_{L}$ is the installation angle of the left detection sensor, and $\varphi$ is the skew angle of the minor road where measured positive if clockwise):

$$
\theta_{n}=\frac{\pi}{2}+\alpha_{L}-\varphi-\arctan \left(\frac{w_{f}}{l-c_{n}}\right)
$$

For a vehicle approaching from the right, the azimuth angle is computed by applying the following equation (where $\alpha_{R}$ is the installation angle of the right detection sensor): 


$$
\theta_{n}=\frac{\pi}{2}+\alpha_{R}+\varphi-\arctan \left(\frac{w_{f}}{l-c_{n}}\right)
$$

The code determines whether the approaching vehicle is within the coverage area of the detection sensor by comparing the azimuth angles with the opening angle of the detection sensor, which is the maximum azimuth angle that could be detected by the sensor (as given by the user based on the technical specifications of the detection sensor used).

Based on the turning signal activated by the driver and input by the user (either left, right or going straight if no signal was activated) and also based on the direction where the bullet vehicle is approaching from, the code determines the conflict type used to compute the time required for the approaching vehicle to reach the intersection, $t_{\text {bullet, }}$ and the time required for the departing target vehicle to clear the path of that approaching vehicle, $t_{\text {target }}$, as per the methodology described in Chapter 4 and illustrated in Figure 4.2.

If an approaching bullet vehicle was found to have no conflict with the departing target vehicle (as shown in Figure 4.2a), the code drops that vehicle from calculations and gives it a 'safe' label. For all other approaching vehicles, the code gives each of them either a 'safe' or 'no safe' label by comparing the time required for that vehicle to reach the intersection, $t_{\text {bullet, }}$ with the time required for the departing target vehicle to clear the path of that approaching vehicle, $t_{\text {target }}$ 
Finally, the code gives a final decision based on the labels attached to all approaching vehicles so that if all the approaching vehicles were found to have no risk of colliding with the departing vehicles, the code gives a 'safe' message.

Two output reports are generated by the code; the first output report, READINGS.txt, includes the range and azimuth angle readings that should be input to the second sensor-simulation MATLAB code. A sample report is shown in Appendix 'D' for the second output report, TWSC_VALID.txt, which typically contains the following:

1. All the input data given to the code (for verification purpose).

2. The perception-reaction time for the driver (based on age and gender as calculated by the statistical regression model discussed in Chapter 5).

3. The distance and speed of the nearest approaching vehicle (to be used to compute driver's correction factor for the maximum acceleration rate of the equipped vehicle as per the statistical regression model discussed in Chapter 5).

4. The correction factor for the maximum acceleration rate of the equipped vehicle.

5. The departure acceleration rate of the equipped vehicle.

6. Range and azimuth angle of every approaching vehicle at different time instances (to be used by the other sensor simulation MATLAB code that will be discussed in this chapter).

7. The time required for every approaching vehicle to reach the intersection, $t_{\text {bullet. }}$ 
8. The time required for the departing target vehicle to clear the path of every approaching vehicle, $t_{\text {target }}$

9. The safety margin associated with every approaching vehicle, which is the difference between the time required for that vehicle to reach the intersection, $t_{\text {bullet}}$, and the time required for the departing target vehicle to clear the path of that approaching vehicle, $t_{\text {target }}$.

10. The safety label given for every approaching vehicle, whether or not it is safe of colliding with the departing vehicle.

11. The final decision made by the system - whether or not to continue the warning signal.

A flow chart is shown in Figure 6.1 for the validation code TWSC_VALID where the symbols in the trapezoids are user inputs while the symbols in the rectangles are outputs of system computations. The following symbols and abbreviations are used in the flow chart:

- POL: The acceleration model used for the departing vehicle

- L: Linear decay acceleration model used for the departing vehicle

- C: Constant acceleration model used for the departing vehicle

- AV: Maximum acceleration provided by the departing vehicle

- EQ: Equilibrium (crawl) speed of the departing vehicle

○ T: Time interval for the detection sensor 


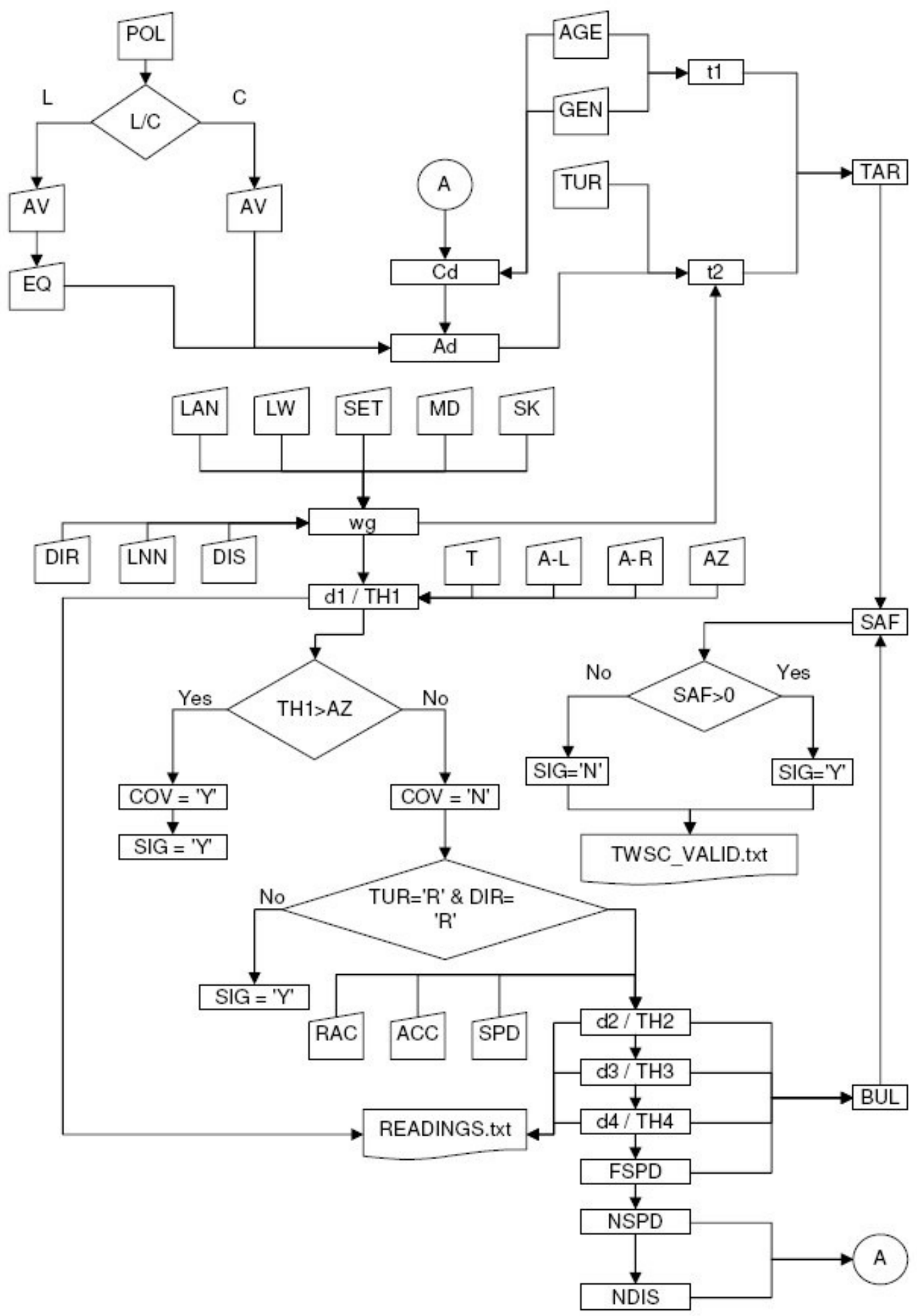

Figure 6.1 Flow chart for the validation code TWSC_VALID 
- A-L: Installation angle for the left detection sensor

- A-R: Installation angle for the right detection sensor

- AZ: Maximum azimuth angle that can be detected by the detection sensor

- AGE: Age of the driver of the departing vehicle

- GEN: Gender of the driver of the departing vehicle

○ TUR: The turning signal activated by the driver of the departing vehicle

- LN: Number of lanes of the major roadway

LW: Width of lane on the major roadway

- SET: Setback distance

○ MD: Median width

- SK: Skew angle between the minor roadway and the major roadway

- DIR: Direction where the approaching vehicle is approaching from

- LNN: Lane number where the approaching vehicle is traveling on

- DIS: Initial distance of the approaching vehicle from the intersection

- SPD: Initial speed of the approaching vehicle

- ACC: Initial acceleration o the approaching vehicle

- RAC: Acceleration rate-of-change for the approaching vehicle

- COV: An indicator to whether the approaching vehicle is out of coverage

- SIG: An indicator to whether a safe signal should be generated

- BUL: Bullet time

- TAR: Target time

- SAF: Safety margin

○ NSPD: Speed of the nearest approaching vehicle 
- NDIS: Distance of the nearest approaching vehicle

- wg: Side offset between the approaching vehicle and the departing vehicle

- Cd: Driver's correction factor for departing vehicle's maximum acceleration

- Ad: Driver's selected acceleration for the departing vehicle

○ t1: Driver's perception-reaction time

- t2: Travel time required for the departing vehicle for a safe departure

- d1: Range reading of the approaching vehicle at first time instance

$\circ$ d2: Range reading of the approaching vehicle at $2^{\text {nd }}$ time instance

$\circ$ d3: Range reading of the approaching vehicle at $3^{\text {rd }}$ time instance

$\circ d 4$ : Range reading of the approaching vehicle at $4^{\text {th }}$ time instance

- TH1: Azimuth angle reading of the approaching vehicle at first time instance

- TH2: Azimuth angle reading of the approaching vehicle at $2^{\text {nd }}$ time instance

- TH3: Azimuth angle reading of the approaching vehicle at $3^{\text {rd }}$ time instance

- TH4: Azimuth angle reading of the approaching vehicle at $4^{\text {th }}$ time instance

- A: Connector symbol

\subsection{Sensor Simulation Code}

The second MATLAB code, TWSC_SIM, is shown in Appendix 'E'. It is based on the principles of trigonometric functions that relate the detection sensor readings together. The user should input the range and azimuth angles for all different approaching vehicles at four consecutive time instances $(T ; T+t ; T+2 t ; T+3 t)$ where $T$ 
is the start time and $t$ is the time interval used for the detection sensors. Those range and azimuth angle readings can be obtained from the first code, TWSC_VALID.

As this code is a simulation for an actual detection sensor, the user should input the user-related, sensor-related and vehicle-related information that should be available for an actual system. The user-related information that should be input includes driver's age and gender as well as the turning signal activated by the driver. The sensor-related information includes installation angle, the time interval used, the opening angle of the detection sensor, and the reflective point of the vehicle detected by the sensor (either the near edge of the vehicle, the centre of the vehicle or the far edge of the vehicle). Finally, the vehicle-related information includes its length, the maximum acceleration rate provided by its engine and the equilibrium speed where the acceleration decreases to zero (the crawl speed).

As this code is a simulation for an actual system, geometric configuration of the roadway is not required as it should not be available for an actual system. The initial conditions of the approaching vehicles (including initial speed, initial acceleration and the rate of change in acceleration) are not also required for that code as they should not be available for an actual system.

A flow chart is shown in Figure 6.2 for the sensor-simulation code TWSC_SIM where the symbols in the trapezoids are user inputs while the symbols in the rectangles are outputs of system computations. It should be noted that the only inputs in this case are the range and azimuth angle readings from the detection sensor at four consecutive time instances (in addition to vehicle, sensor and driver information that are pre-input to the system). 


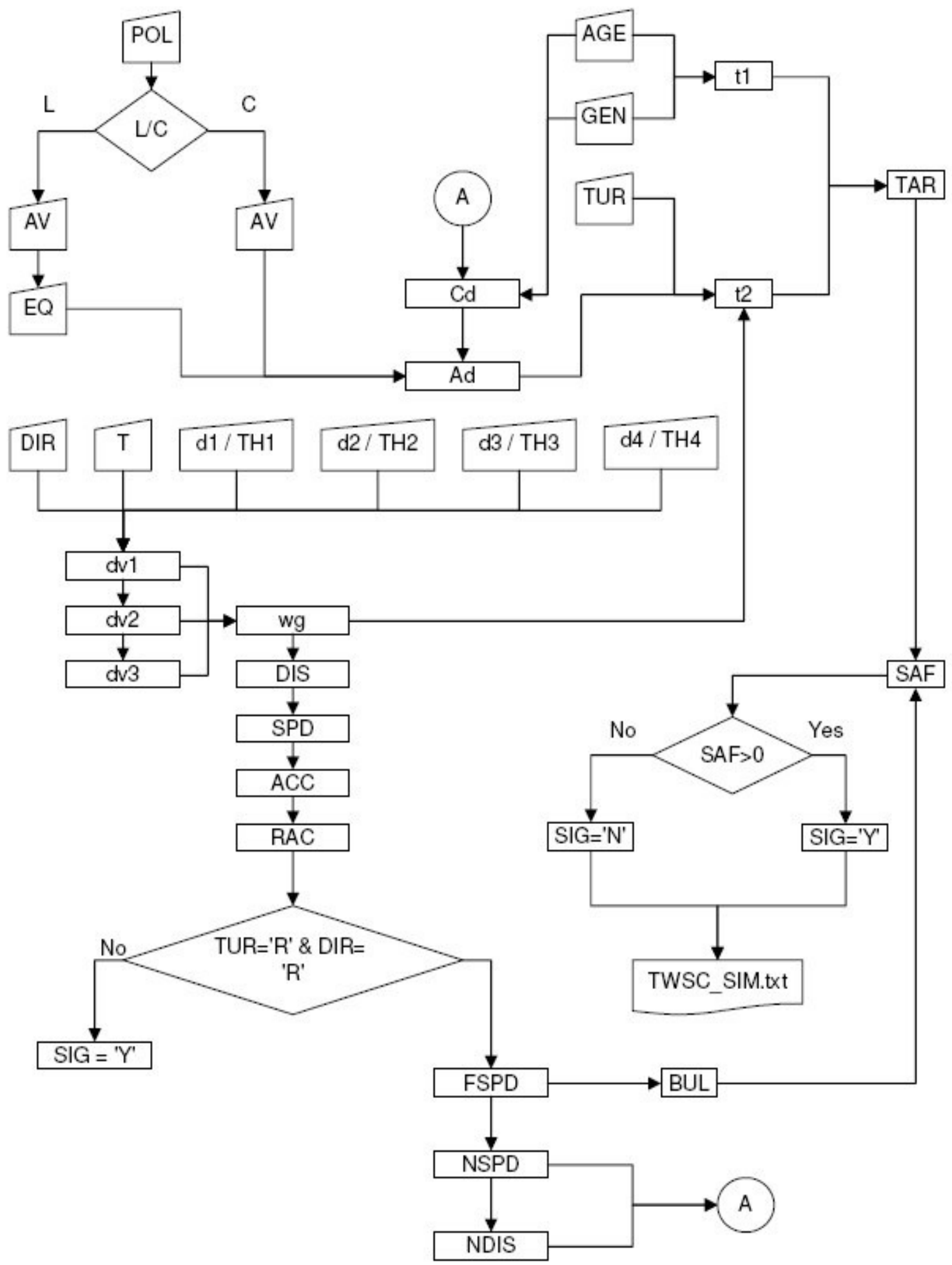

Figure 6.2 Flow chart for the sensor-simulation code TWSC_SIM 
From the sensor readings, the code estimates the initial conditions for every approaching vehicle as per the methodology discussed in Chapter 4. Based on the turning signal activated by the driver and input by the user (either left, right or going straight if no signal was activated) and also based on the direction where the bullet vehicle is approaching from, the code determines the conflict type used to compute the time required for the approaching vehicle to reach the intersection, $t_{\text {bullet, }}$ and the time required for the departing target vehicle to clear the path of that approaching vehicle, $t_{\text {target}}$, as per the methodology described in Chapter 4 and illustrated in Figure 4.2. If an approaching bullet vehicle was found to have no conflict with the departing target vehicle (as shown in Figure 4.2a), the code drops that vehicle from calculations and gives it a 'safe' label. For all other approaching vehicles, the code gives each of them either a 'safe' or 'no safe' label by comparing the time required for that vehicle to reach the intersection, $t_{\text {bullet, }}$ with the time required for the departing target vehicle to clear the path of that approaching vehicle, $t_{\text {target }}$. Finally, the code gives a final decision based on the labels attached to all approaching vehicles so that if all the approaching vehicles were found to have no risk of colliding with the departing vehicles, the code displays a 'safe' message to the driver.

An output report, TWSC_SIM.txt, is generated by the code. A sample report is shown in Appendix 'F', which typically contains the following:

1. All the input data given to the code (for verification purpose).

2. The perception-reaction time for the driver (based on age and gender as calculated by the statistical regression model discussed in Chapter 5). 
3. The distance and speed computed by the code for the nearest approaching vehicle (to be used to compute driver's correction factor for the maximum acceleration rate of the equipped vehicle as per the statistical regression model discussed in Chapter 5).

4. The correction factor for the maximum acceleration rate of the equipped vehicle.

5. The departure acceleration rate of the equipped vehicle.

6. The initial conditions for every approaching vehicle as computed by the code, including initial speed, initial acceleration and the rate of change in acceleration.

7. The time required for every approaching vehicle to reach the intersection, $t_{\text {bullet. }}$

8. The time required for the departing target vehicle to clear the path of every approaching vehicle, $t_{\text {target }}$

9. The safety margin associated with every approaching vehicle, which is the difference between the time required for that vehicle to reach the intersection, $t_{\text {bullet, }}$ and the time required for the departing target vehicle to clear the path of that approaching vehicle, $t_{\text {target }}$.

10. The safety label given for every approaching vehicle, whether or not it is safe of colliding with the departing vehicle.

11. The final decision made by the system - whether or not to continue the warning signal. 
The results of both codes were compared together to verify the accuracy of all the algorithm equations. For instance, comparing the initial conditions for a specific approaching vehicle as computed by the second code, TWSC_SIM, with the initial conditions for the same vehicle as given by the user for the first code, TWSC_VALID, should give an indication about the accuracy of the algorithm equations and methodology. Both codes were also used to assess the impact of different components on the functionality of the system. The following sections provide more information on the validation and sensitivity analysis.

\subsection{Effect of Measurement Precision}

Measurement precision of the detection sensors is a key factor in the success of the system. Although the selection of the physical components and hardware used in the system is not a part in the scope of this research, the developed simulation MATLAB codes can be useful in assessing the impact of measurement precision of the detection sensors on the accuracy of the system. To assess the impact of measurement precision, a series of different scenarios were simulated by the first validation code, TWSC_VALID, which can precisely simulate the system with no measurement error. The same scenarios were also simulated using the second sensor-simulation code, TWSC_SIM, with different levels of reading precision. To keep the proposed system as realistic as possible, a range reading precision of 0.05 $m$ was used when assessing the effect of angle precision, and an angle reading precision of $0.1^{\circ}$ was used when assessing the effect of range reading precision. 
These values are selected as they are the values recommended by previous research to ensure acceptable level of accuracy with keeping the system realistic (NHTSA 2000; US DOT 2003; NHTSA 2004).

The results for the effect of the range measurement precision on computing the bullet vehicle time and safety margin for a vehicle approaching on the nearest lane with different approaching speeds are shown in Figure 6.3 and Figure 6.4, respectively. Both figures show that measurement precision is affected by the speed of the approaching vehicle as the larger speed reduces the relative error due to the larger displacement distances detected at the same time interval. For a typical approaching speed of $60 \mathrm{~km} / \mathrm{h}$, a measurement precision $5 \mathrm{~cm}$ or better should give satisfactory results as the error sharply increases if the measurement precision is poorer than $5 \mathrm{~cm}$. This finding is consistent with the findings of earlier research (NHTSA 2000; US DOT 2003; NHTSA 2004).

The results for the effect of the azimuth angle measurement precision on the bullet vehicle time and safety margin for a vehicle approaching on the nearest lane with different approaching speeds are shown in Figure 6.5 and Figure 6.6, respectively. Both figures show that the accuracy of the system is more sensitive to the precision of measuring the azimuth angle. For a typical approaching speed of 60 $\mathrm{km} / \mathrm{h}$, the accuracy sharply improves when the precision of the azimuth angle is $0.1^{\circ}$ or better, which is also consistent with the findings of earlier research (NHTSA 2000; US DOT 2003; NHTSA 2004). 


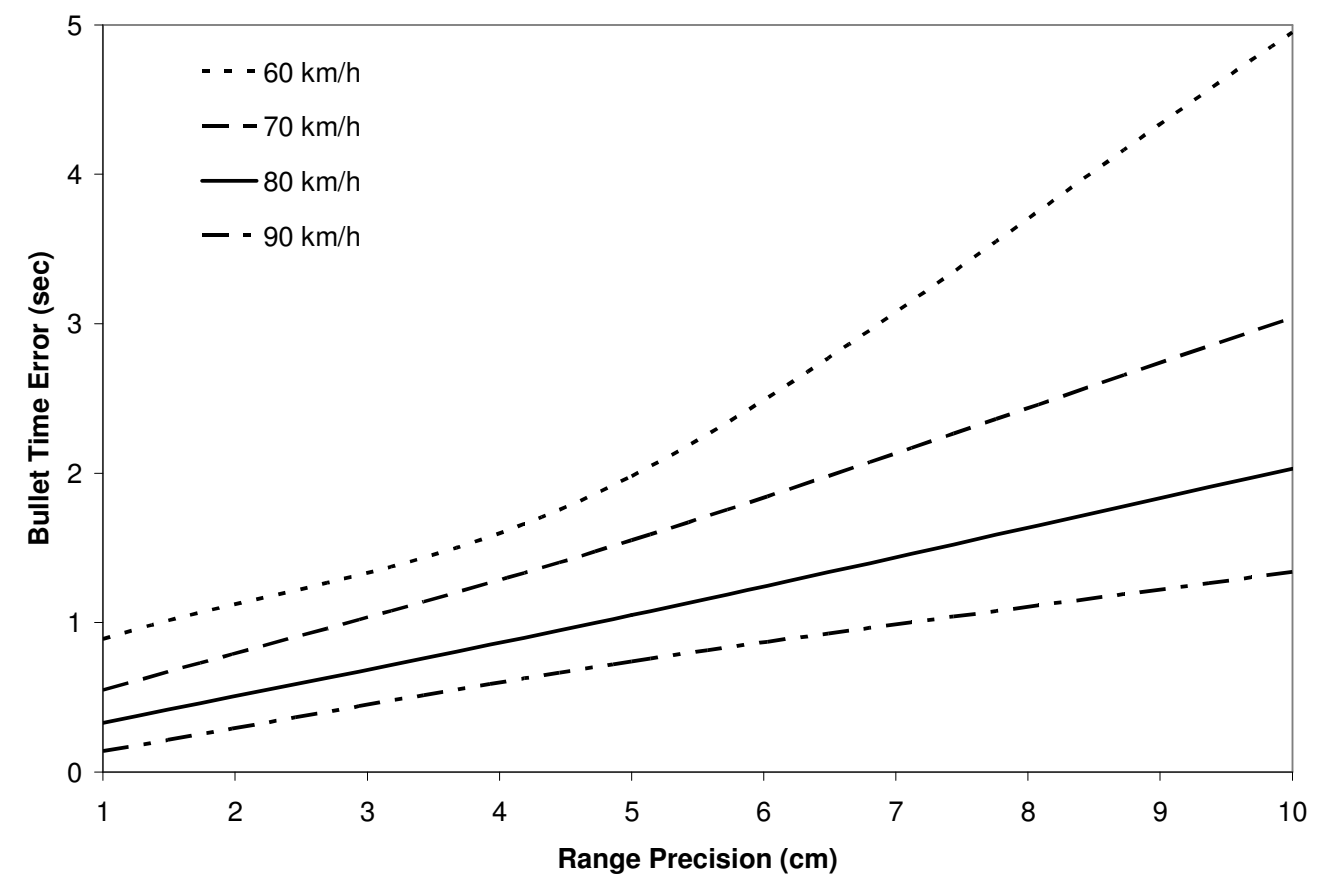

Figure 6.3 Effect of range measuring precision on the bullet time error

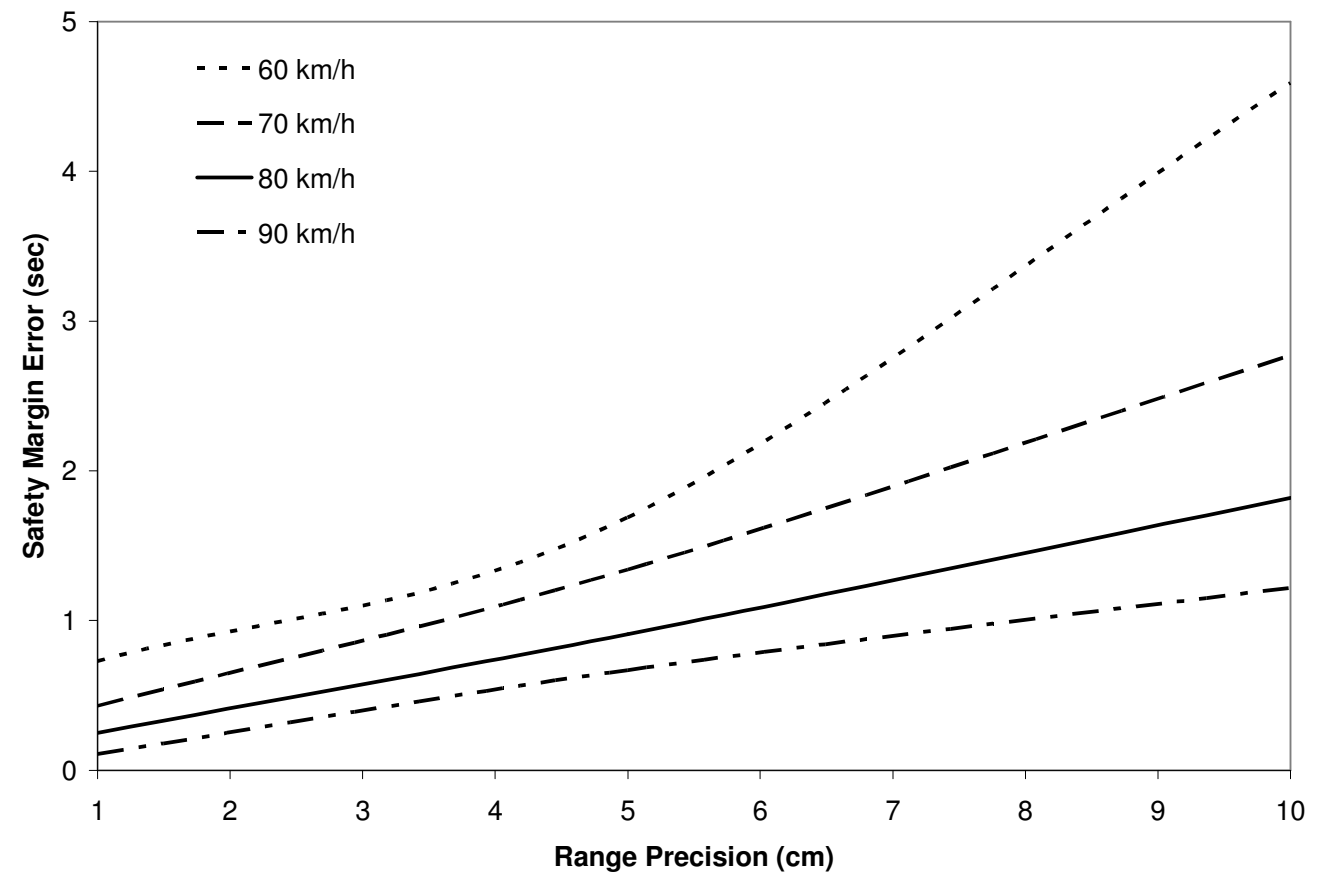

Figure 6.4 Effect of range measuring precision on the safety margin error 


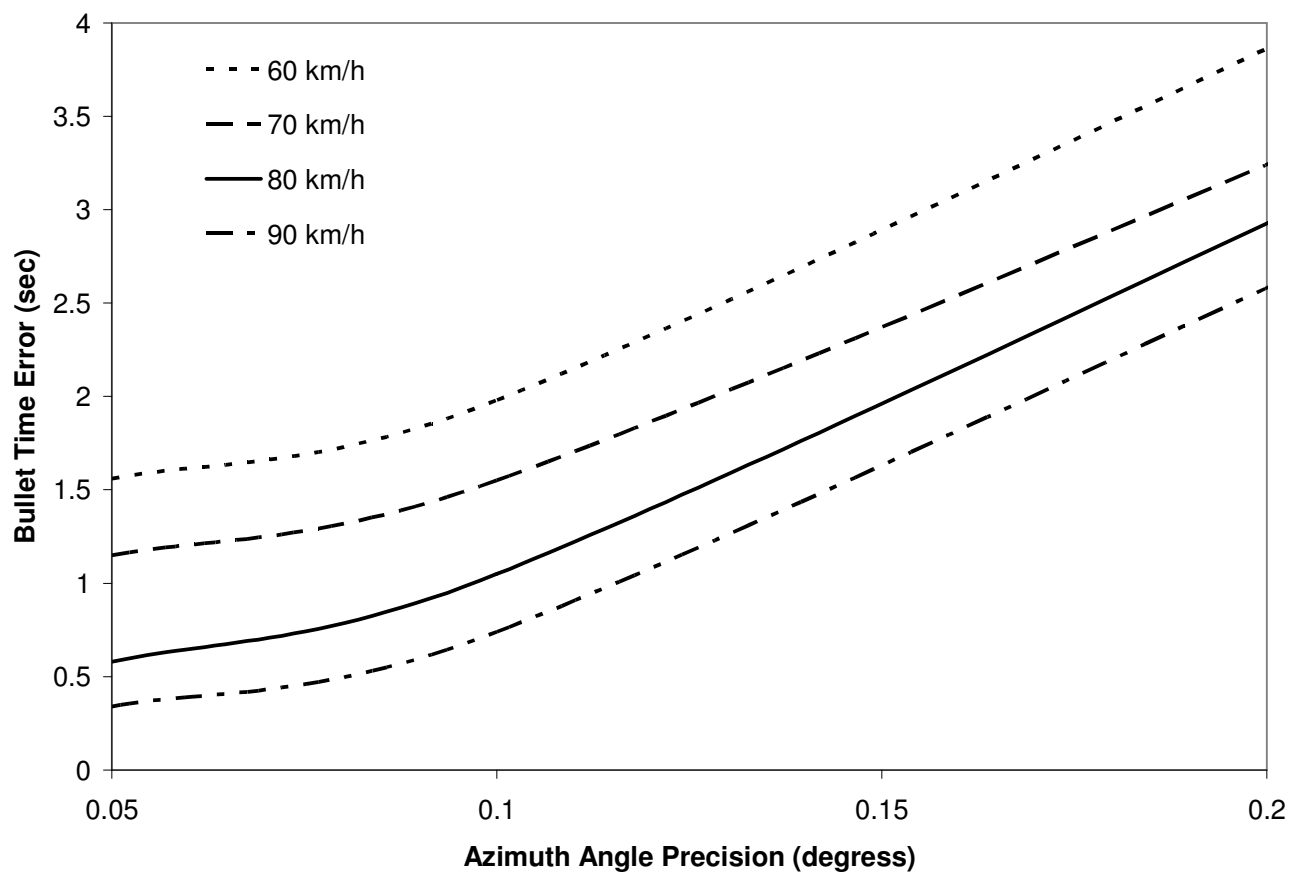

Figure 6.5 Effect of azimuth angle measuring precision on the bullet time error

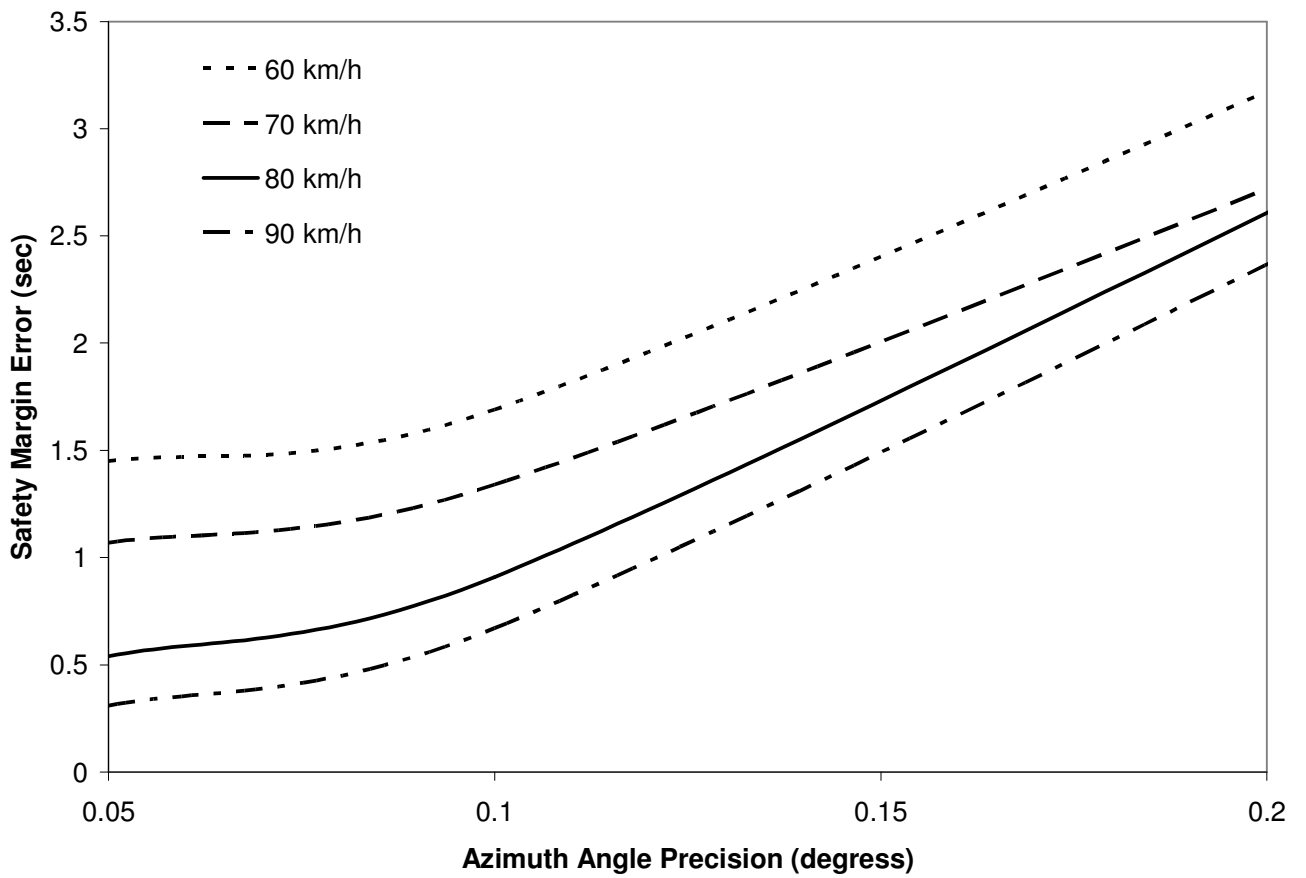

Figure 6.6 Effect of azimuth angle measuring precision on the safety margin error 


\subsection{Effect of Side Offset}

The side offset between the bullet and target vehicles, $w_{f}$, has two impacts on the accuracy of the system. First, the error in reading the side offset would affect the departure time (in the departure cases shown in Figure 4.2b). Moreover, side offset is used as a criterion in deciding whether the approaching vehicle is traveling on the near lane (when the target vehicle is turning right or left in semi-controlled intersections) so that the case shown in Figure 4.2c should apply, or whether the approaching vehicle is traveling on a far lane with no conflict with the departing target vehicle (as shown in Figure 4.2a). The side offset is a function of the following factors:

- For vehicles approaching from the left, the side offset is a function of lane width and setback; and

- For vehicles approaching from the right, the side offset is also a function of lane width and setback as well as in median width.

From Equation 3.22 or Equation 4.23, the side offset is computed from the detection sensor readings as follows:

$$
w_{f}=\frac{1}{3} \sum_{n=1}^{3} \frac{d_{n} d_{n+1} \sin \left(\theta_{n}-\theta_{n+1}\right)}{\sqrt{d_{n}^{2}+d_{n+1}^{2}-2 d_{n} d_{n+1} \cos \left(\theta_{n}-\theta_{n+1}\right)}}
$$


From the above equation, the side offset is sensitive to errors in reading both the range and the azimuth angle. The simulation MATLAB codes were used to assess the impact of measurement precision on the accuracy of computing side offset by simulating a series of different scenarios using the first validation code, TWSC_VALID, which can precisely simulate the system with no measurement error. The same scenarios were also simulated using the second sensor-simulation code, TWSC_SIM, with different levels of reading precision. The results are shown in Table 6.1, which indicates that using $0.05 \mathrm{~m}$ precision for measuring the range and $0.1^{\circ}$ precision for measuring the azimuth angle will likely result in computing the side offset with some acceptable range of accuracy (approximately $1.13 \mathrm{~m}$ when detecting a vehicle approaching from the left on the near lane, and $0.60 \mathrm{~m}$ when detecting a vehicle approaching from the right on the near lane).

Table 6.1 Effect of measuring precision on the side offset error

\begin{tabular}{|c|c|c|c|c|c|}
\hline \multirow{2}{*}{$\begin{array}{c}\text { Vehicle } \\
\text { Approaching } \\
\text { from }\end{array}$} & $\begin{array}{c}\text { Range } \\
\text { Precision }\end{array}$ & $\begin{array}{c}\text { Azimuth Angle } \\
\text { Precision }\end{array}$ & \multicolumn{3}{|c|}{ Side Offset } \\
\cline { 4 - 6 } & $(m)$ & & $\begin{array}{c}\text { Computed } \\
(\text { degree })\end{array}$ & $\begin{array}{c}\text { Actual } \\
(m)\end{array}$ & $\begin{array}{c}\text { Error } \\
(m)\end{array}$ \\
\hline Left & 0.01 & 0.05 & 3.78 & 3.5 & 0.28 \\
Left & 0.05 & 0.05 & 3.96 & 3.5 & 0.46 \\
Left & 0.10 & 0.05 & 4.15 & 3.5 & 0.65 \\
& & & & & \\
Right & 0.01 & 0.05 & 16.64 & 16.75 & 0.11 \\
Right & 0.05 & 0.05 & 16.93 & 16.75 & 0.18 \\
Right & 0.10 & 0.05 & 17.17 & 16.75 & 0.42 \\
& & & & & \\
Left & 0.01 & 0.1 & 4.42 & 3.5 & 0.92 \\
Left & 0.05 & 0.1 & 4.63 & 3.5 & 1.13 \\
Left & 0.10 & 0.1 & 4.95 & 3.5 & 1.45 \\
& & & & & \\
Right & 0.01 & 0.1 & 16.52 & 16.75 & 0.23 \\
Right & 0.05 & 0.1 & 17.35 & 16.75 & 0.60 \\
Right & 0.10 & 0.1 & 17.89 & 16.75 & 1.14 \\
\hline
\end{tabular}




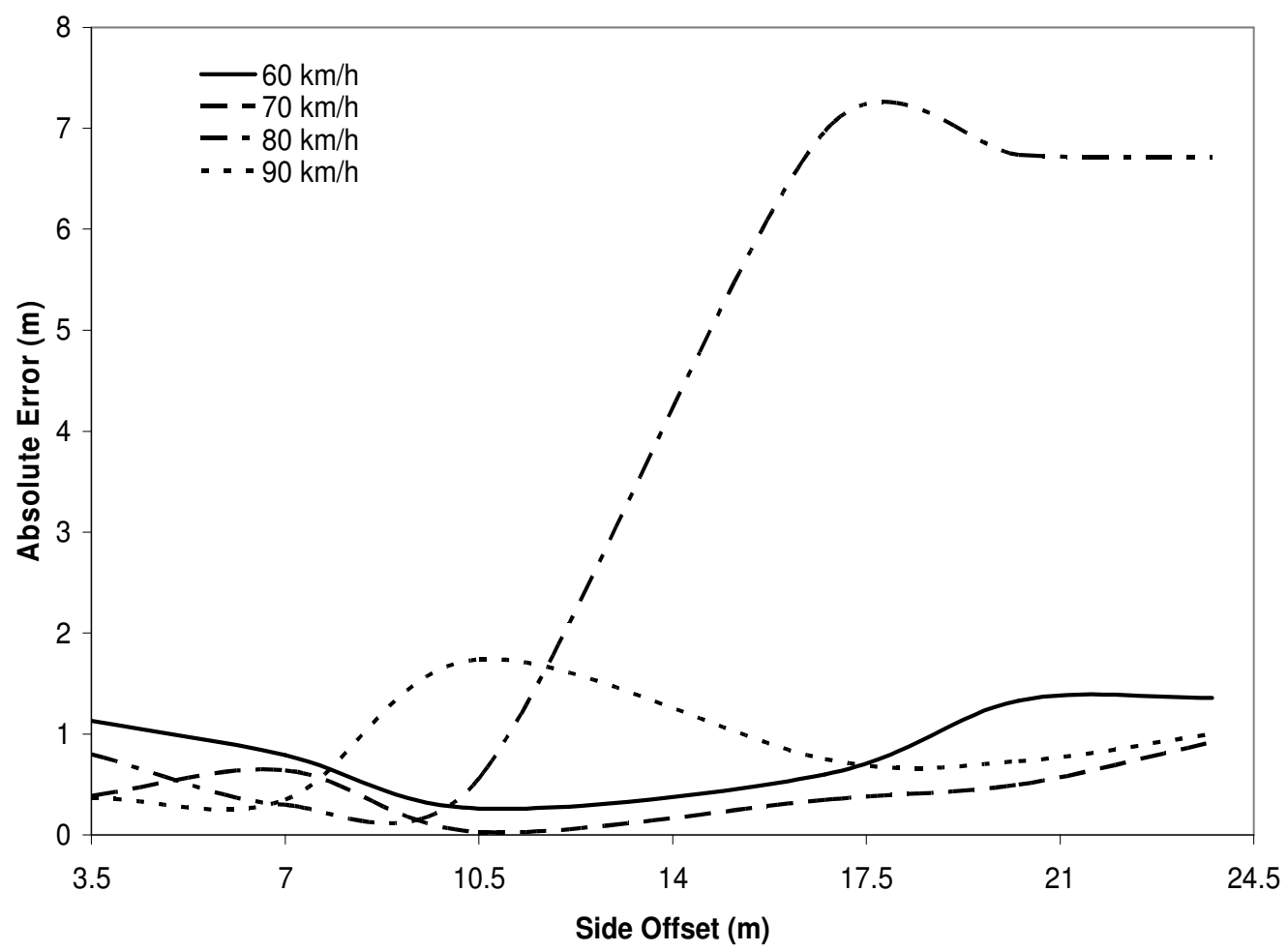

Figure 6.7 Effect of side offset and approaching speed on the reading error

As shown in Figure 6.7, the error in computing the side offset largely depends on the level of precision for every individual reading with no apparent trend. However, the error curve for different approaching speeds has the shape of a sine curve with some trend to increase with the decrease of the approaching speed.

Computing the side offset between the target and bullet vehicles with an acceptable level of confidence is vital to improving the reliability of the system by reducing unnecessary warnings in situations where the target vehicle is turning right or left while the bullet vehicle is approaching from the opposite direction on a further lane (as shown in Figure 4.2a). When the target vehicle is turning right while the bullet vehicle is approaching from left, and to ensure that the approaching vehicle is 
not traveling on the same near lane that the target vehicle is turning into, the side offset should be greater than the width of a typical lane plus a typical setback in addition to the correction factor for the distance between the reflective point of the approaching vehicle and its nearest edge. The lane width typically ranges between $3.00 \mathrm{~m}$ to $3.75 \mathrm{~m}$, and the setback distance typically ranges between $1.00 \mathrm{~m}$ to 1.75 m (AASHTO 2001; TAC 1999). Based on that, the typical offset distance, $w_{f}$, for a vehicle not traveling on the near lane should be as follows:

$\circ$ between $4.00 \mathrm{~m}$ and $5.50 \mathrm{~m}$ in case when the reflective point detected in that vehicle is at the vehicle's near edge;

$\circ$ between $5.06 \mathrm{~m}$ and $6.56 \mathrm{~m}$ in case when the reflective point detected in that vehicle is at the centre of the vehicle; and

$\circ$ between $6.13 \mathrm{~m}$ and $7.63 \mathrm{~m}$ in case when the reflective point detected in that vehicle is at the vehicle's far edge.

Tables 6.2 to 6.4 show the minimum and maximum values for the side offset distances for vehicles not traveling on the near lane where the reflective point is located at the near edge, the centre and at the far edge, respectively. These tables are based on the reading errors found in Figure 6.7. For a certain detected approaching speed, if the side offset value computed by the system was found to be greater than the maximum value shown in Tables 6.2 to 6.4 , depending on the location of the reflective point, it could be concluded with high level of confidence that the approaching vehicle is not traveling on the near lane; moreover, if the side offset value computed by the system was found to be less than the minimum value shown 
in Tables 6.2 to 6.4 , depending on the location of the reflective point, it could be concluded with the same high level of confidence that the approaching vehicle is traveling on the near lane. If the side offset computed by the system, for a certain detected approaching speed, was found to be within the ranges shown in Tables 6.2 to 6.4 , it will not be feasible to predict the actual side offset with an acceptable level of confidence; and therefore, it will not be possible to determine whether the detected vehicle is traveling on the near lane or not. A conservative approach is to assume that the detected approaching vehicle is traveling on the near lane unless the computed side offset was found to be greater than the maximum values shown in Tables 6.2 to 6.4 , depending on the location of the reflective point, that are associated with the approaching speed of that vehicle as detected by the system. For instance, if the reflective point is at the near edge and a vehicle was detected to be traveling with a speed $70 \mathrm{~km} / \mathrm{h}$ at a computed side offset $5.25 \mathrm{~m}$, which is less than the maximum value of $6.11 \mathrm{~m}$ shown in Table 6.2, that vehicle is assumed to be traveling on the near lane with its path conflicting with the departing target vehicle.

Table 6.2 Side offset for a vehicle on far lane (reflective point at near edge)

\begin{tabular}{ccccc}
\hline $\begin{array}{c}\text { Approaching } \\
\text { Speed }\end{array}$ & $\begin{array}{c}\text { Side Offset } \\
(m)\end{array}$ & $\begin{array}{c}\text { Error } \\
(m)\end{array}$ & $\begin{array}{c}\text { Minimum Value } \\
(m)\end{array}$ & $\begin{array}{c}\text { Maximum Value } \\
(m)\end{array}$ \\
\hline $60 \mathrm{~km} / \mathrm{h}$ & $4.00-5.50$ & $1.08-0.99$ & 2.92 & 6.49 \\
$70 \mathrm{~km} / \mathrm{h}$ & $4.00-5.50$ & $0.48-0.61$ & 3.52 & 6.11 \\
$80 \mathrm{~km} / \mathrm{h}$ & $4.00-5.50$ & $0.80-0.66$ & 3.20 & 6.16 \\
$90 \mathrm{~km} / \mathrm{h}$ & $4.00-5.50$ & $0.40-0.39$ & 3.60 & 5.89 \\
\hline
\end{tabular}


Table 6.3 Side offset for a vehicle on far lane (reflective point at centre)

\begin{tabular}{ccccc}
\hline $\begin{array}{c}\text { Approaching } \\
\text { Speed }\end{array}$ & $\begin{array}{c}\text { Side Offset } \\
(m)\end{array}$ & $\begin{array}{c}\text { Error } \\
(m)\end{array}$ & $\begin{array}{c}\text { Minimum Value } \\
(m)\end{array}$ & $\begin{array}{c}\text { Maximum Value } \\
(m)\end{array}$ \\
\hline $60 \mathrm{~km} / \mathrm{h}$ & $5.06-6.56$ & $1.03-0.86$ & 4.03 & 7.42 \\
$70 \mathrm{~km} / \mathrm{h}$ & $5.06-6.56$ & $0.64-0.59$ & 4.42 & 7.15 \\
$80 \mathrm{~km} / \mathrm{h}$ & $5.06-6.56$ & $0.66-0.32$ & 4.40 & 6.88 \\
$90 \mathrm{~km} / \mathrm{h}$ & $5.06-6.56$ & $0.26-0.29$ & 4.80 & 6.85 \\
\hline
\end{tabular}

Table 6.4 Side offset for a vehicle on far lane (reflective point at far edge)

\begin{tabular}{ccccc}
\hline $\begin{array}{c}\text { Approaching } \\
\text { Speed }\end{array}$ & $\begin{array}{c}\text { Side Offset } \\
(m)\end{array}$ & $\begin{array}{c}\text { Error } \\
(\%)\end{array}$ & $\begin{array}{c}\text { Minimum Value } \\
(m)\end{array}$ & $\begin{array}{c}\text { Maximum Value } \\
(m)\end{array}$ \\
\hline $60 \mathrm{~km} / \mathrm{h}$ & $6.13-7.63$ & $0.94-0.74$ & 5.19 & 8.37 \\
$70 \mathrm{~km} / \mathrm{h}$ & $6.13-7.63$ & $0.73-0.48$ & 5.40 & 8.11 \\
$80 \mathrm{~km} / \mathrm{h}$ & $6.13-7.63$ & $0.58-0.19$ & 5.55 & 7.82 \\
$90 \mathrm{~km} / \mathrm{h}$ & $6.13-7.63$ & $0.41-0.49$ & 5.72 & 8.12 \\
\hline
\end{tabular}

The second case is when the target vehicle is turning left while the bullet vehicle is approaching from the right. To ensure that the approaching vehicle is not traveling on the same near lane that the target vehicle is turning into, the side offset should be greater than the sum of the setback distance, the width of all travel lanes on the near side of the road, the median width and the width of one lane on the far side of the road. Due to the large variation in the value of all those components (setback, lane width, median width, and number of lanes), it is not practically possible to verify, with an acceptable level of confidence, that the approaching vehicle is not traveling on the near lane; and therefore, the conservative approach is to always assume that the approaching vehicle is traveling on the near lane with its path conflicting with the departing target vehicle. 


\subsection{Effect of Hidden Vehicles}

There is a probability that a small vehicle (e.g. a motorcycle) may be hidden from the detection sensor by another larger vehicle (e.g. a transportation truck) during all time intervals used for detection. In the worst case, the hidden small vehicle would accelerate to gain a speed that is larger than the speed of the detected vehicle while it is still hidden from the detection sensors during all time intervals used for detection. This situation may be critical when the system concludes that the detected vehicle is not likely to collide with the departing target vehicle, while the speed and acceleration of the hidden vehicle are so large that it may collide with the departing target vehicle.

An example for the above situation is when a detected vehicle is approaching from the left on the near (first) lane at a distance $125 \mathrm{~m}$, speed $60 \mathrm{~km} / \mathrm{h}(16.667$ $\mathrm{m} / \mathrm{sec}$ ), acceleration rate $0.25 \mathrm{~m} / \mathrm{sec}^{2}$, and rate of change of acceleration 0.05 $\mathrm{m} / \mathrm{sec}^{3}$. By applying the methodology described in Chapter 4 , the anticipated time for the detected vehicle to reach the intersection, $t_{\text {bullet, }}$ should be 6.2 seconds. Assuming that the driver of the departing target vehicle is 36 years old male and the maximum acceleration rate provided by the mechanical characteristics of the target vehicle is $3.85 \mathrm{~m} / \mathrm{sec}^{2}$ with a crawl speed of $40 \mathrm{~m} / \mathrm{sec}$ and assuming that the reflective point is at the far edge, the time required for the target vehicle to clear the path of the approaching vehicle, $t_{\text {target}}$, should be 3.9 seconds, which is less than the anticipated time for the detected vehicle to reach the intersection. However, there may be a hidden vehicle on a further (second or third) lane that is traveling on a higher speed without being detected by the sensors. If the speed of the hidden vehicle is $90 \mathrm{~km} / \mathrm{h}$ 
( $25 \mathrm{~m} / \mathrm{sec}$ ), its anticipated time to reach the intersection would be 4.3 seconds, which is less than the time required for the target vehicle to clear the path of that hidden vehicle (4.4 seconds). This means that the detected vehicle was found to have no risk of colliding with the departing target vehicle while there is a risk of collision with the hidden vehicle that is not detected by the detection sensors.

The simulation MATLAB codes were used to examine the effect of hidden vehicles on the reliability of the system. Based on the findings of earlier research (Fitzpatrick et al. 2003), the probability for a hidden vehicle to be traveling on high speed is as follows:

- $15 \%$ probability (1 in 7 hidden vehicles) to be traveling on a speed higher than $96.6 \mathrm{~km} / \mathrm{h}$ (60 mph);

- $5 \%$ probability (1 in 20 hidden vehicles) to be traveling on a speed higher than $109.5 \mathrm{~km} / \mathrm{h}$ (68 mph); and

- $2 \%$ probability ( 1 in 50 hidden vehicles) to be traveling on a speed higher than $114.3 \mathrm{~km} / \mathrm{h}(71 \mathrm{mph})$.

Based on the level of risk selected, there is a critical distance where if a hidden vehicle is traveling within that critical distance, it may constitute a risk of colliding with the departing target vehicle even if the detected vehicle (traveling on the nearer lane) was found to have no risk of colliding with the target vehicle. That critical distance depends on many factors, including the lane on which the hidden vehicle is traveling, the lane width, the setback distance, driver's age and gender (to calculate perception-reaction time), the reflective point detected by the sensor, and the 
mechanical characteristics of the departing vehicle. The MATLAB simulation codes were used to establish the chart shown in Figure 6.8, which is based on the following assumptions:

- Driver is 28 years old male;

- Equipped vehicle has a maximum acceleration rate of $4.50 \mathrm{~m} / \mathrm{sec}^{2}$ and a crawl speed of $40 \mathrm{~m} / \mathrm{sec}$;

- The reflective point detected in the approaching vehicle is at the near edge; and

○ Setback distance is $1.75 \mathrm{~m}$, and the lane width is $3.50 \mathrm{~m}$.

The chart shown in Figure 6.8 can be used to calculate the critical distance at different risk levels under the above specific assumptions. For instance, if the target vehicle is turning left into a major road that has two lanes in each direction, a hidden vehicle may be traveling on the second lane (with side offset approximately $7 \mathrm{~m}$ ) and it may collide with the departing target vehicle if another detected vehicle (on the near lane) was detected at a distance that is less than the following critical distances (associated with different levels of risk):

○ $136 \mathrm{~m}$ (if 1-in-7 hidden vehicles risk level was used);

○ $154 \mathrm{~m}$ (if 1-in-20 hidden vehicles risk level was used); or

○ $161 \mathrm{~m}$ (if 1-in-50 hidden vehicles risk level was used).

If a vehicle was detected on a distance that is less than the distance shown above (depending on the level of risk used), there might be a risk of having a hidden vehicle 
on the further lane that may collide with the departing target vehicle even if the detected vehicle was found to be safe of colliding with the departing target vehicle. It should be noted that this problem persists either with or without an intersection collision warning system. The proposed system, as well as all preceding systems, is not intended to address this problem as the detection sensors currently available in the market are not capable of detecting hidden vehicles. The main objective of the proposed intersection warning system is to compensate for the human error in perceiving the speed and acceleration of approaching vehicles, which means that it should not be considered as an aid for drivers to detect vehicles that are not visible to them.

The usual practice for most drivers is that they do not depart a controlled intersection if a large vehicle is approaching and they simply wait until that large vehicle passes and then they start waiting for another time gap to make their departure. A similar course of action may be taken by drivers who have their vehicles equipped with the proposed warning system so that they may simply wait until any large vehicle passes and then they start waiting for another safe signal from the system to make their departure. 


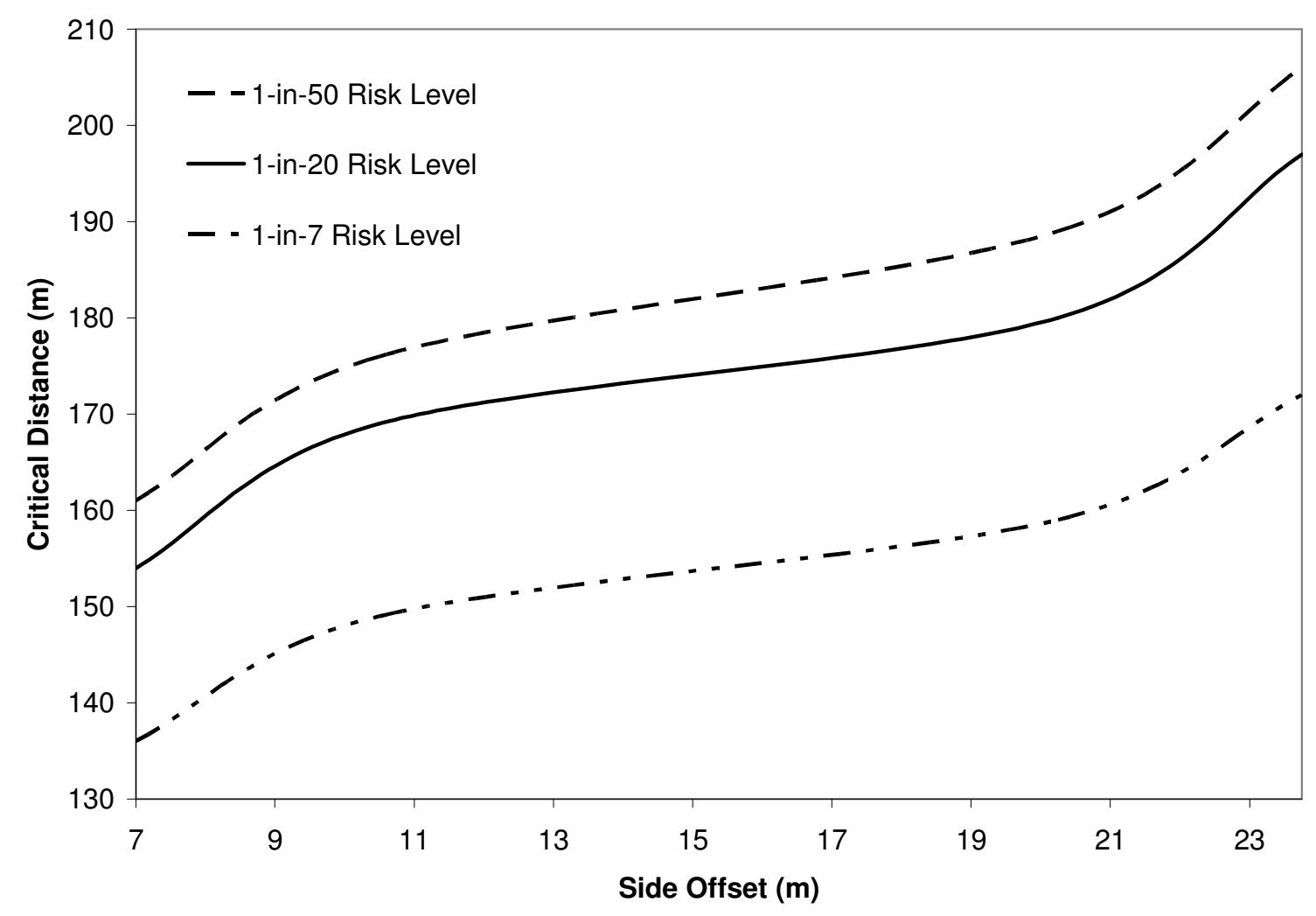

Figure 6.8 Effect of side offset on the critical distance for hidden vehicles

\subsection{Effect of Acceleration Model}

The acceleration models assumed for both the target and bullet vehicles may also influence the accuracy of the system. As was discussed in Chapter 3, the bullet vehicles are usually travelling on near-constant speeds and also given the small time intervals used by the system, the approaching vehicles are assumed to be travelling on variable acceleration with a linear relationship between the time, $t$, and the rate of change in the acceleration, $r$. As for the target vehicle, it is starting from a stop position; and therefore, its acceleration is assumed to be decreasing with the 
increase of the speed as was observed by earlier researchers (Drew 1968; Long 2000). Changing the acceleration model for either the bullet or the target vehicle may affect the accuracy of the system as will be explained in the following sections.

\subsubsection{Bullet Vehicle Acceleration Model}

The acceleration model selected for the bullet vehicle is that the acceleration of the vehicle at any time has a linear relationship with the time. Based on that, the system has to take four consecutive measurements to create three equations that should be mutually solved to obtain the initial conditions; namely the initial acceleration, $a_{T}$, the initial speed, $v_{T}$, and the rate of change in acceleration, $r$. If the approaching vehicle was assumed to have a constant rate of acceleration, then only two equations, instead of three, should be mutually solved to obtain the initial conditions (constant acceleration, $a$ and the initial speed, $v_{T}$ ) as the rate of change in acceleration would be assumed to be zero. That assumption may impact the accuracy of the system but it may also reduce the processing time by one time interval, $t$, as only three consecutive readings would be required (instead of four).

Based on the above, a third MATLAB code has been developed, TWSC_CONST, which is shown in Appendix 'G'. That code assumes a constant acceleration rate for the bullet vehicles and the results from that code were compared to those from the original code, TWSC_VALID, to assess the error resulted from assuming a constant acceleration rate for the bullet vehicles. 
If the approaching vehicle has $v_{T}$ initial speed, at time $T$, and a constant acceleration rate, $a$, the speed at the end of the first time interval can be computed from the following equation:

$$
v_{T+t}=v_{T}+a t
$$

Similarly, the speed at the end of the second time interval can be computed from the following equation:

$$
v_{T+2 t}=v_{T}+2 a t
$$

The displacement during the first time interval can be computed as follows:

$$
d v_{1}=v_{T} t+\frac{1}{2} a t^{2}
$$

Similarly, the displacement during the second time interval can be computed as follows:

$$
d v_{2}=v_{T+t} t+\frac{1}{2} a t^{2}=\left(v_{T}+a t\right) t+\frac{1}{2} a t^{2}=v_{T} t+\frac{3}{2} a t^{2}
$$


By subtracting Equation 6.8 from Equation 6.9, the acceleration rate can be computed as follows:

$$
a=\frac{d v_{2}-d v_{1}}{t^{2}}
$$

By substituting for the acceleration rate in either Equation 6.8 or Equation 6.9, the initial speed is computed as follows:

$$
v_{T}=\frac{3 d v_{1}-d v_{2}}{2 t}
$$

In the above calculations, the displacements $d v_{1}$ and $d v_{2}$ are both computed from the readings at three consecutive time intervals similar to what was discussed in Chapter 3 and Chapter 4 . The offset distance between the bullet and target vehicles is computed from the following equation:

$$
w_{f}=\frac{\sum_{n=1}^{2} d_{n+1} \sin \beta_{n+1}}{2}=\frac{\sum_{n=1}^{2} d_{n+1} \frac{d_{b}}{d_{v n}} \sin \left(\theta_{n}-\theta_{n+1}\right)}{2}=\frac{1}{2} \sum_{n=1}^{2} \frac{d_{n} d_{n+1} \sin \left(\theta_{n}-\theta_{n+1}\right)}{\sqrt{d_{n}^{2}+d_{n+1}^{2}-2 d_{n} d_{n+1} \cos \left(\theta_{n}-\theta_{n+1}\right)}}
$$

The final distance between the approaching vehicle and the intersection, $d_{f}$, at the end of the second time interval is computed from the following equation: 


$$
d_{f}=\sqrt{d_{3}^{2}-w_{f}^{2}}
$$

Finally, the anticipated time for the bullet vehicle to reach the intersection, $t_{\text {bullet }}$, is the real positive root for the following polynomial equation (where $d_{f}$ is calculated from Equation 6.13, $v_{T+2 t}$ is calculated from Equation 6.7, and $a$ is calculated from Equation 6.10):

$$
d_{f}=v_{T+2 t} t_{\text {bullet }}+\frac{1}{2} a t_{\text {bullet }}^{2}
$$

To assess the impact of the acceleration model for the approaching vehicles on the accuracy of the system readings, a series of different scenarios were simulated by the first validation code, TWSC_VALID, which can precisely simulate the system and calculate anticipated time to reach the intersection, $t_{b u l l e t}$, by assuming variable acceleration rate. The same scenarios were also simulated using the third MATLAB simulation code, TWSC_CONST, with incorporating the above methodology into it so that the code should determine the anticipated time for the bullet vehicle to reach the intersection, $t_{\text {bullet, }}$ by assuming a constant acceleration rate.

By comparing the results obtained from both MATLAB codes, it was found that the rate of change in acceleration has virtually no effect on computing the side offset. The effect on computing the bullet time and the safety margin is shown in Figure 6.9 and Figure 6.10, respectively, which show that the error increases with the decrease of the initial acceleration due to the higher relative impact of the error on smaller 
acceleration rates. It should be noted that the values shown for the rate of change in acceleration are negative values as it is expected that the vehicles detected are moving on relatively high speeds (not starting from stop) and therefore their rate of change in acceleration is decreasing as per the Linear Decay model recommended by earlier research (Drew 1968; Long 2000).

The maximum error was found to be approximately 2.25 seconds, which is associated with an acceleration rate of $0.25 \mathrm{~m} / \mathrm{sec} / \mathrm{sec}$ and a rate of change in acceleration $-1.25 \mathrm{~m} / \mathrm{sec} / \mathrm{sec}$. For larger acceleration rates, the error is within 0.5 seconds. However, it should be noted that for bullet vehicles that are moving on relatively high speeds (not starting from stop), the acceleration rate is expected to be relatively small and therefore, the error resulting from using constant acceleration model may be significant and exceeds the saving in processing time associated with using the constant acceleration model (where only two time intervals are needed instead of three).

Based on the above, using a variable acceleration model (with three time intervals) may improve the overall accuracy of the system as the benefit gained (in terms of improved accuracy) exceeds the cost (in terms of extending the processing time by an extra time interval).

\subsubsection{Target Vehicle Acceleration Model}

As the target vehicle typically starts from a stop position, the acceleration model selected for it is the Linear Decay model, which assumes that the acceleration rate 
decreases with the increase of the speed of the vehicle. This assumption is the most realistic representation of actual driving situations; however, many design guides (AASHTO 2001; TAC 1999) assume a constant rate of acceleration when starting from a stop position. The MATLAB validation code, TWSC_VALID, has been used to assess the error resulting from using a constant acceleration model for the target vehicle.

A series of scenarios have been simulated using a constant acceleration model and the same scenarios have been simulated again using the Linear Decay acceleration model. The comparison results for the two groups are shown in Table 6.5, which shows that for perpendicular conflict types shown in Figure 4.2b (e.g. when the target vehicle is turning left while the bullet vehicle is approaching from the left) the effect of the acceleration model on the computation of the target time is less than 0.11 seconds and can be neglected with no significant impact on the accuracy of the system. However, for the conflict type where both the target and bullet vehicles are traveling on the same lane in the same direction as shown in Figure 4.2c (e.g. when the target vehicle is turning right while the bullet vehicle is approaching from the left) the effect of the acceleration model on the computation of the target time is much more significant due to the larger distance required for the target vehicle to accelerate in order to avoid a collision.

As was discussed in Chapter 4, for the case of the second conflict type, the target vehicle should accelerate to gain $70 \%$ of the speed of the bullet vehicle and the distance traversed during the acceleration period is typically more than $100 \mathrm{~m}$, depending on the factors shown in Equation 4.40. 


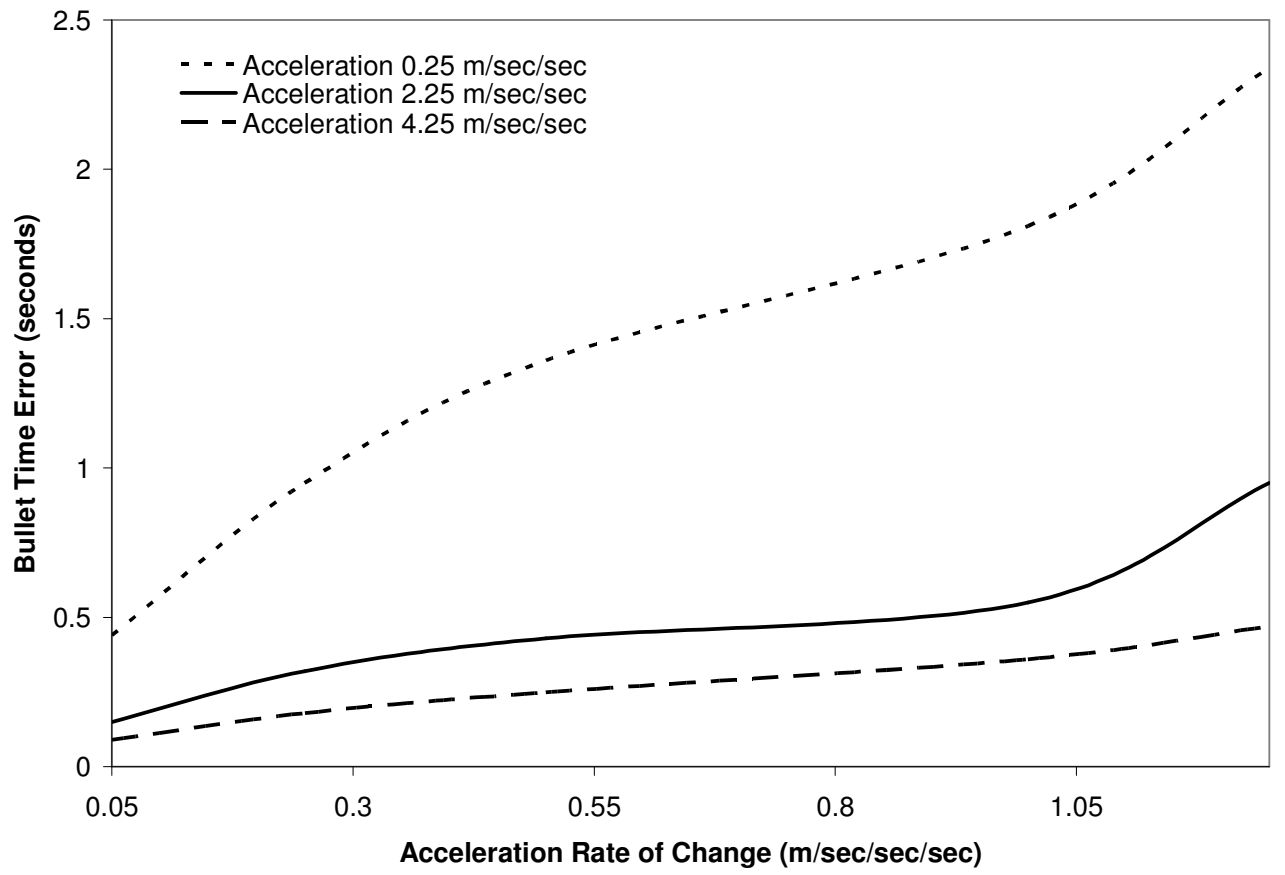

Figure 6.9 Effect of bullet vehicle acceleration model on computing bullet time

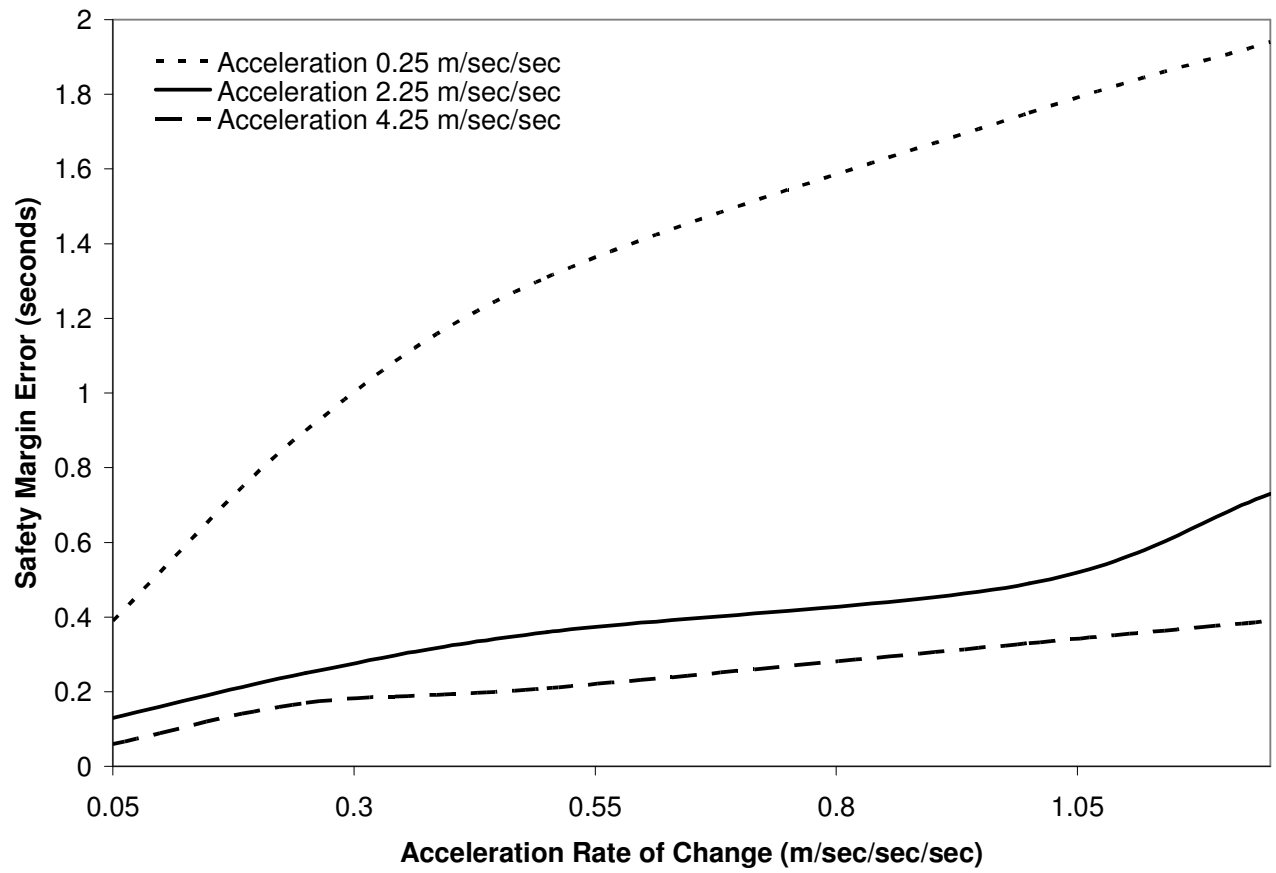

Figure 6.10 Effect of bullet vehicle acceleration model on computing safety margin 
Table 6.5 Effect of acceleration model on target time computation

\begin{tabular}{|c|c|c|c|c|c|}
\hline \multirow[t]{2}{*}{ Conflict Type } & \multirow{2}{*}{$\begin{array}{l}\text { Acceleration } \\
(\mathrm{m} / \mathrm{s} / \mathrm{s})\end{array}$} & \multirow{2}{*}{$\begin{array}{c}\text { Crawl Speed } \\
(\mathrm{m} / \mathrm{s})\end{array}$} & \multicolumn{3}{|c|}{ Target Time } \\
\hline & & & Observed & Actual & Error \\
\hline \multirow[t]{15}{*}{ Perpendicular } & \multirow[t]{5}{*}{1.5} & 25 & 5.65 & 5.76 & 0.11 \\
\hline & & 30 & 5.65 & 5.74 & 0.09 \\
\hline & & 35 & 5.65 & 5.73 & 0.08 \\
\hline & & 40 & 5.65 & 5.72 & 0.07 \\
\hline & & 45 & 5.65 & 5.71 & 0.06 \\
\hline & \multirow[t]{5}{*}{3} & 25 & 4.40 & 4.51 & 0.11 \\
\hline & & 30 & 4.40 & 4.49 & 0.09 \\
\hline & & 35 & 4.40 & 4.47 & 0.07 \\
\hline & & 40 & 4.40 & 4.46 & 0.06 \\
\hline & & 45 & 4.40 & 4.45 & 0.05 \\
\hline & \multirow[t]{5}{*}{4.5} & 25 & 3.84 & 3.95 & 0.11 \\
\hline & & 30 & 3.84 & 3.93 & 0.09 \\
\hline & & 35 & 3.84 & 3.92 & 0.08 \\
\hline & & 40 & 3.84 & 3.91 & 0.07 \\
\hline & & 45 & 3.84 & 3.90 & 0.06 \\
\hline \multirow[t]{15}{*}{ Same Lane } & \multirow[t]{5}{*}{1.5} & 25 & 20.24 & 47.84 & 27.60 \\
\hline & & 30 & 20.24 & 35.71 & 15.47 \\
\hline & & 35 & 20.24 & 31.29 & 11.05 \\
\hline & & 40 & 20.24 & 28.90 & 8.66 \\
\hline & & 45 & 20.24 & 27.37 & 7.13 \\
\hline & \multirow[t]{5}{*}{3} & 25 & 10.81 & 24.61 & 13.80 \\
\hline & & 30 & 10.81 & 18.54 & 7.73 \\
\hline & & 35 & 10.81 & 16.33 & 5.52 \\
\hline & & 40 & 10.81 & 15.13 & 4.32 \\
\hline & & 45 & 10.81 & 14.37 & 3.56 \\
\hline & \multirow[t]{5}{*}{4.5} & 25 & 7.66 & 16.86 & 9.20 \\
\hline & & 30 & 7.66 & 12.82 & 5.16 \\
\hline & & 35 & 7.66 & 11.35 & 3.69 \\
\hline & & 40 & 7.66 & 10.55 & 2.89 \\
\hline & & 45 & 7.66 & 10.04 & 2.38 \\
\hline
\end{tabular}

For comparison, for the first perpendicular conflict type, the distance required for the target vehicle to accelerate is typically the side offset between the target and bullet vehicles plus the length of the target vehicle itself as well as the correction factor for the distance between the reflective point detected by the sensor and the far 
edge of the approaching vehicle. This distance is typically less than $20 \mathrm{~m}$. Due to the large difference between the distances required for the target vehicle to accelerate in both cases, the effect of the acceleration model on the accuracy of the target time computation is more significant for the case shown in Figure 4.2c and may impact the accuracy of the whole system. Therefore, selecting the linear decay model for the acceleration profile of the target vehicle will result in improving the accuracy, and consequently reliability, of the system especially in situations where both the bullet and target vehicles are traveling on the same lane at the same direction. 


\section{CHAPTER 7: SUMMARY, CONCLUSIONS AND RECOMMENDATIONS}

This chapter provides summary and conclusions related to the research work presented followed by an outline for the scientific contributions made by this research as well as the limitations of the proposed system. Finally recommendations for future research work are presented.

\subsection{Summary and Conclusions}

This research presented the design and validation procedures for an intersection collision warning system that has the potential of improving safety and reducing intersection collisions by assisting drivers in selecting proper gaps at intersections. The system utilizes detection sensors (either radar sensors or laser scanners) to detect different vehicles traveling on the opposite/cross-traffic lanes and determines their speeds and acceleration rates as well as their rate of change in acceleration. Based on that, the developed system's algorithm can estimate the expected time required for every approaching vehicles to reach the intersection. The system also estimates the time required for the equipped vehicle to clear the intersection and by comparing the two times, the system displays a message for the driver of the equipped vehicle when it is safe to start departure. This will help reduce the human errors related to the driver of the equipped vehicle by avoiding the misjudgement related to perceiving the speeds and acceleration rates of the approaching vehicles. 
The time required for the equipped vehicle to depart the intersection depends on its intended path of departure as well as on the time required for the driver to perceive the message received from the system and react to it. Departure time also depends on the driver's desired rate of acceleration when departing the intersection. Initial regression models have been developed to estimate the driver's perceptionreaction time (that is based on age and gender) and departure acceleration rate (that is based on age, gender, and the speed and location of the nearest approaching vehicle). Those models have been developed from data collected from a sample of 60 drivers who proportionally represent the population of registered drivers in both Ontario and Canada in terms of age and gender. It should be noted that the sample size selected may not be fully representative to the population in terms of driving behaviour, especially with the lack of statistical parameters of the population with respect to the phenomena described by the statistical models. However, it was impractical to substantially increase the sample size, especially with the lack of the statistical parameters of the population that leads to uncertainty in determining the required sample size that produces an acceptable margin of error between the sample and the population. The sample size selected was within the same range of the sample sizes selected by similar previous driver simulation studies.

Every driver in the sample was asked to drive six different simulation scenarios on the STISIM driving simulator located at Ryerson University. The first three scenarios were designed to simulate a series of left turn movements, and the other three 
scenarios were designed to simulate different departure movements (turning left, turning right and crossing) from controlled minor roadways into non-controlled major roadways. The developed algorithms were encoded into the scenarios so that at each intersection the subject would hear a buzz signal indicating that it was not safe to depart the intersection, and was instructed to depart the intersection as soon as the buzz signal stops for that intersection. The experimental design gave consideration to control for variations in geometric configurations as well as variations in mechanical characteristics of different vehicles.

The developed regression models have been validated by asking a sample of 6 licensed drivers, who were not included in the sample used to calibrate the models, to drive the same simulation scenarios on the STISIM driving simulator. To validate the statistical models over all different ages, the ages of the drivers used to validate the models were different from the ages used in calibrating the models. The sample size selected for the validation was found to be associated with a margin of error between the calibration and validation samples that is insignificant for all calibrated statistical models. It was also found that the sum of the validation errors is small for all the models, which indicates that the errors resulting from validating the models fluctuate around zero and do not accumulate to a negative or a positive trend. It was also found that the mean squared validation error for all the models was insignificant and the root mean squared errors of validation was also very small (less than 0.24 seconds in calculating $t_{1}$, and less than $2.24 \%$ in calculating the correction factor for acceleration rates). Furthermore, the root mean squared errors, $R M S E_{v}$, were found to be very close to the standard errors associated with model calibration, indicating 
that the models are stable and robust when being validated for predictors that were not used in calibrating the models.

The above initial regression models would be useful at the early implementation of the warning system by individual drivers. To improve the reliability of the proposed warning system over the time, a methodology was introduced to continually record the actual perception-reaction times and acceleration rates of each specific driver on a specific vehicle and use the created database to calibrate nonlinear prediction models using artificial neural networks, ANN, that replace the original regression models. Four different ANN models have been developed using MATLAB codes where the first two models predict driver's perception-reaction time for left turn situations and semi-controlled intersections, respectively; while the other two models predict driver's desired acceleration rate for left turn situations and semi-controlled intersections, respectively. The developed ANN models have been validated by using the database created from experimental observations of 60 drivers used to develop the original linear regression models. The idea was to use part of the dataset for every driver to 'train' the appropriate ANN model, and then validate the model by comparing the predicted dependent variables to their corresponding observed actual values in the remaining part of the original dataset. It was found that the predicted values were close to the observed values with a small root-mean-squared-error, which demonstrates that the developed ANN models are robust and yield reasonable values with acceptable errors. It should be noted that the artificial neural network models should not replace the initial regression models until the size of the dataset 
created by the system produces root-mean-squared error values that are less than those for the original linear regression models.

The proposed system have been validated by coding the system into two MATLAB codes where the first code estimates positions, speeds, and acceleration rates of all approaching vehicles at different time instances from the laws of dynamic motion; while the second code estimates them by applying the principles of trigonometric functions on the detection sensor readings at four consecutive time instances. The results of both codes were compared together to verify the accuracy of all the algorithm equations. Both codes were also used to assess the impact of different components on the functionality and reliability of the system. The impact of measurement precision of the detection sensors have been assessed where a series of different scenarios were simulated by the first MATLAB validation code, which can precisely simulate the system with no measurement error. The same scenarios were simulated again using the second MATLAB simulation code with different levels of reading precision, and it was found that for a typical approaching speed of $60 \mathrm{~km} / \mathrm{h}$, a range measurement precision of $0.05 \mathrm{~m}$ and an azimuth angle measurement precision of $0.1^{\circ}$ should give satisfactory results with acceptable error as the computation error sharply increases for poorer measurement precision.

Computing the side offset between the departing and approaching vehicles with an acceptable level of confidence is vital to improving the reliability of the system by reducing unnecessary warnings in situations where the departing vehicle is turning right while the approaching vehicle is approaching from left on a further lane. Different thresholds have been established by setting minimum values for the side 
offset that depends on the speed of the approaching vehicle as well as on the location of the reflective point detected by the sensors used by the system. Those thresholds account for the variations in setback distance and lane width as well as for the reading error. If the side offset computed by the system was found to exceed the threshold established, it could be concluded with high level of confidence that the approaching vehicle is not traveling on the near lane and therefore it has no conflict with the turning vehicle. Those thresholds can only be applied to the situation where the departing vehicle is turning right while the approaching vehicle is approaching from left. For other situations where the departing vehicle is turning left while the approaching vehicle is approaching from the right, it is not practically possible to verify, with an acceptable level of confidence, that the approaching vehicle is not traveling on the near lane. This is due to the large variation in the value of all the components that comprise the side offset for that case (setback, lane width, median width, and number of lanes).

The simulation MATLAB codes, developed in this research, were used to examine the probability that a small vehicle may be hidden from the detection sensor by another larger vehicle during all time intervals used for detection and that the hidden vehicle may gain a speed that is larger than the speed of the detected vehicle while it is still hidden from the detection sensors. Different thresholds have been established, depending on the level of risk selected, to identify the critical distance where if a hidden vehicle is traveling within that critical distance, it may constitute a risk of colliding with the departing target vehicle even if the detected vehicle (traveling on the nearer lane) was found to have no risk of colliding with the target vehicle. This 
problem of hidden vehicles persists either with or without an intersection collision warning system, and it should be noted that the proposed system, as well as all preceding systems, is not intended to address this problem as the detection sensors currently available in the market are not capable of detecting hidden vehicles. The usual practice for most drivers is that they do not depart a controlled intersection if a large vehicle is approaching and they simply wait until that large vehicle passes and then they start waiting for another time gap to make their departure. A similar course of action may be taken by drivers who have their vehicles equipped with the proposed warning system so that they may simply wait until any large vehicle passes and then they start waiting for another safe signal from the system to make their safe departure.

The simulation MATLAB codes, developed in this research, were also used to assess the impact of the acceleration model for the approaching vehicles on the accuracy of the system readings. Two cases have been investigated where the first case was to assume a constant acceleration rate for the approaching vehicles while the second case was to assume time-dependent variable acceleration rate for the approaching vehicles. The first case, where a constant acceleration rate is assumed, requires less processing time as only three consecutive sensor readings are required, while the second case, which is more realistic representation for actual vehicle acceleration, requires four consecutive sensor readings. While the first case requires less processing time (by saving one reading interval), it was found that the error associated with it exceeds the time savings and therefore, using a variable acceleration model (where four consecutive sensor readings are required) may 
improve the overall accuracy of the system as the benefit gained (in terms of improved accuracy) exceeds the cost (in terms of extending the processing time by an extra time interval).

Finally, the MATLAB simulation codes, developed in this research, were also used to assess the error resulting from assuming constant acceleration for the target vehicle (instead of the realistic linear decay acceleration where the acceleration decrease with the increase of the speed) and it was found that for perpendicular conflict types the effect of assuming constant acceleration on the computation of the target time is insignificant, while for the conflict type where both the departing and approaching vehicles are traveling on the same lane in the same direction the effect of assuming constant acceleration on the computation of the target time is more significant due to the larger distance required for the target vehicle to accelerate in order to avoid a collision.

\subsection{Research Contributions}

Below is a list of the key scientific contributions made by this research:

1. Designing the algorithms that compute the anticipated times for approaching vehicles to reach an intersection and compare those times to the required time for equipped vehicles to depart that intersection and making a decision whether or not to display a warning message to the driver of the equipped vehicle. Different algorithms have been designed for different intersection situations that rely on gap-acceptance criterion where a driver has to perceive 
the situation and select a proper gap in the opposite/cross traffic stream to start departure. To increase the accuracy in calculating the anticipated times for approaching vehicles to reach an intersection, the developed algorithms take into account the speed, acceleration and rate-of-change in acceleration for all the approaching vehicles. To increase the accuracy in calculating the required time for equipped vehicles to depart an intersection, the developed algorithms take into account the perception-reaction time required for the drivers of the equipped vehicles to perceive the message displayed by the warning system and react to it; furthermore, the developed algorithms also take into account the variations among drivers in selecting the desired acceleration rate when departing an intersection. Those developed algorithms can be applied to any intersection collision warning system to increase its accuracy and reliability.

2. Developing and validating statistical models that estimate the perceptionreaction times required for the driver of any vehicle equipped with an intersection collision warning system to perceive the message displayed by the warning system and react to it. This includes the initial regression models that should only be applied at the early stage of implementing the warning system as well as the more-sophisticated artificial neural network models that are based on actual data collected from a specific driver on a specific vehicle so that they should produce better estimate for future perception-reaction times of that specific driver. Those statistical models can also be applied to 
any intersection collision warning system to increase its accuracy and reliability.

3. Developing and validating statistical models that estimate the acceleration rate selected by a specific driver when departing an intersection using the gapacceptance criterion. This includes the initial regression models that should only be applied at the early stage of implementing the warning system as well as the more-sophisticated artificial neural network models that are based on actual data collected from a specific driver on a specific vehicle so that they should produce better estimate for future acceleration rates selected by that specific driver. Similar to the perception-reaction time models, those statistical models can also be applied to any intersection collision warning system to increase its accuracy and reliability.

4. Examining the impact of different factors on the reliability of an intersection collision warning system, including the impact of reading precision as well as the acceleration profiles assumed for both the turning and approaching vehicles. Conclusions have been made to select the feasible reading precision that produces acceptable computation error, which was consistent with earlier research findings. Conclusions have also been made to select the acceleration profiles for both the turning and approaching vehicles that minimize computation errors and increase the accuracy and reliability of the warning system.

5. Establishing different thresholds for the side offset that accounts for the variations in setback distance and lane width as well as for the reading error, 
where if the side offset computed by an intersection warning system was found to exceed the threshold established, it could be concluded with high level of confidence that the detected vehicle is not traveling on the near lane and therefore it has no conflict with the turning vehicle in case if the detected vehicle is approaching from left and the equipped vehicle is turning right. This will eliminate unnecessary warnings and increase the reliability of any warning system by reducing driver's nuisance.

\subsection{System Limitations}

The sample size selected to calibrate the initial regression models may not be fully representative of the population in terms of driving behaviour, especially with the lack of statistical parameters of the population with respect to the phenomena described by the statistical models. However, it was impractical to substantially increase the sample size, especially with the lack of the statistical parameters of the population that leads to uncertainty in determining the required sample size that produces an acceptable margin of error between the sample and the population. The sample size selected was within the same range of the sample sizes selected by similar previous driver simulation studies.

It should also be noted that using driving simulator to collect the data used to calibrate the initial regression models might not be fully representative to actual driving behaviour; however, using driving simulator was the only 
feasible method to collect the data in the early stage prior to building a prototype that may be used in later stages to collect more realistic data based on actual driving experience. It should be noted that the regression models developed from the sample size used are only applicable at the early stage of implementing the system and they should be later replaced by more-realistic self-learning artificial neural network models that are also developed in this research.

The proposed system is not designed for situations where an intersection collision results from driver's violation of traffic by-laws. An example is at a TWSC intersection where a driver of an equipped vehicle (on the minor road) attempts to enter the major road without fully stopping at the stop sign. In this case, the equipped vehicle might collide with another vehicle traveling on the major road as a result of driver's aggressive behaviour rather than from his/her misjudgement. Proper law enforcement measures would be more effective in addressing this type of collisions.

It should also be noted that the main objective of the proposed intersection warning system is to compensate for the human error in perceiving the speed and acceleration of approaching vehicles, which means that it should not be considered as an aid for drivers to detect vehicles that are not visible to them, either as a result of poor intersection sight distance or in other situations where a large vehicle blocks the sightline between the detection sensors and another small vehicle that is traveling on a further lane. Successful implementation of the proposed warning system would 
require that the intersections have adequate sight distances in order for the system to detect the approaching vehicles.

\subsection{Future Research Directions}

Further research will be required to cover aspects related to different engineering disciplines such as electrical and mechanical engineering. This includes selecting or manufacturing different hardware components of the system, building a prototype to be tested at different levels including field testing. Field testing should be the most effective method of validating the system and examining its feasibility. Field testing will also be useful in collecting more realistic data that can be used to update the initial regression models to estimate the perception-reaction time for the driver of the equipped vehicle as well as the selected acceleration rate when departing an intersection. After testing the system, a before-and-after study may be required to examine the benefits of the system in terms of reduced intersection collisions and deciding whether those benefits justify the cost of the system. Further research and development will be required to achieve those goals, which are beyond the scope of this research and should be achieved using multi-discipline approach where other researchers should be involved from different engineering disciplines.

The self-learning artificial neural network models have the potential to reduce the uncertainty in estimating human factors related to the proposed system over the time due to the ever-increasing size of the database used to calibrate the models. However, if scientific evidence proved that human errors 
will be significant even after implementing the self-learning artificial neural network models in an actual system, statistical observations may be collected at that stage (after building an actual system) to produce more realistic estimates for the error in human factors; and the system algorithms may be revised at that stage to account for those error estimates. 


\section{References}

Akcelik, R. and Biggs, D. C. (1987). Acceleration profile model for vehicles in road traffic. Transportation Science. Vol. 21(1), pp. 36-54.

Alexander, G. J. (1989). Search and perception-reaction time at intersections and railroad grade crossings. Institute of Transportation Engineers Journal. Vol. 59(11), pp. 17-20.

Alexander, J., Barham, P. and Black, I. (2002). Factors influencing the probability of an incident at a junction: results from an interactive driving simulator. Accident Analysis and Prevention Vol. 34, pp. 779-792.

Allen, R. W., Park, G., Rosenthal, T. J. and B. L. Aponso (2004). A process for developing scenarios for driving simulations. Presented at IMAGE 2004 Conference, Scottsdale, AZ, USA.

American Association of State Highway and Transportation Officials (AASHTO) (2001). A Policy on Geometric Design of Highways and Streets (The Green Book), $4^{\text {th }}$ Edition. Washington, D.C., USA.

American Automobile Association (1958). Reaction time as related to age. Research Report No.69. American Automobile Association, Washington, D.C., USA.

Bella, F. (2005).Validation of a driving simulator for work zone design. Transportation Research Record 1937, pp. 136-144, Transportation Research Board, National Research Council, Washington, D.C., USA. 
Bella, F., Garcia, A., Solves, F., Romero, M.A., 2007. Driving simulator validation for deceleration lane design. Proc. $86^{\text {th }}$ Annual Meeting of the Transportation Research Board, Washington, D.C., USA.

Bellis, W. R. (1960). Capacity of Traffic Signals and Traffic Signal Timing. Bulletin 271, pp. 45-67, HRB, National Research Council, Washington, D.C., USA.

Belz, S. M., Robinson, G. S., and Casali, J. G. (1998). Auditory icons as impending collision system warning signals in commercial motor vehicles. Proceedings of the Human Factors and Ergonomics Society 42nd Annual Meeting. HFES: Santa Monica, CA, USA.

Belz, S. M., Robinson, G. S., and Casali, J. G. (1999). A New Class of Auditory Warning Signals for Complex Systems: Auditory Icons. Human Factors, Vol. 41(4), pp. 608-618.

Bishop, C. (1995). Neural Networks for Pattern Recognition. Clarendon Press, Oxford, UK.

Blaauw, G. J. (1982). Driving experience and task demands in simulator and instrumented car: a validation study. Human Factors, Vol. 24(3), pp. 473486.

Boer, E. R. (1999). Car-following from the driver's perspective. Transportation Research Part F, National Research Council, Washington, D.C. USA.

Bonneson, J. A. (1990). Operational Characteristics of the Single-Point Urban Interchange. Ph. D. dissertation. Texas A\&M University, College Station, TX, USA. 
Bonneson, J. A. (1992). Modeling Queued Driver Behavior at Signalized Junctions. Transportation Research Record 1365, pp. 99-107, Transportation Research Board, National Research Council, Washington, D.C., USA.

Brown, Timothy L.; Lee, John D.; McGehee, Daniel V (2002). Attention-Based Model of Driver Performance in Rear-End Collisions. Transportation Research Record 1724, Transportation Research Board, National Research Council, Washington, D.C., USA.

Centre for Transportation Research and Education, lowa State University (2004). Impact of Left-Turn Phasing on Older and Younger Drivers at High-Speed Signalized Intersections. Project 03-149. Centre for Transportation Research and Education, lowa State University, IW, USA.

Chakroborty, P. and Kikuchi, S. (1999). Evaluation of the General Motors based Car-Following Models and a Proposed Fuzzy Inference Model. Transportation Research, Part C. Vol. 7(4), pp. 209-235. National Research Council, Washington, D.C., USA.

Chang L. Y. (2005). Analysis of freeway accident frequencies: Negative binomial regression versus artificial neural network. Journal of Safety Science, Vol. 43(8), pp. 541-557.

Dabbour, E. and Easa, S. (2009). Perceptual Framework for a Modern Left-Turn Collision Warning System. International Journal of Applied Science, Engineering and Technology. Vol. 5(1), pp. 8 -14. 
Dabbour, E. and Easa, S. (2009). Technology-Independent Algorithm for Collision Warning System at Semi-Controlled Intersections. Canadian Journal of Transportation [in review].

Dabbour, E., Easa, S. M. and Hossain, K. M. (2009). Explicit Modeling of Human Factors for Intersection Collision Warning Systems. Journal of Advanced Transportation [in review].

Dahl, R. (2004). Vehicular Manslaughter: The Global Epidemic of Traffic Deaths. Environmental Health Perspectives, Vol. 112(11), pp. 628-631.

Davis, G.A. and Swenson, T. (2004). Field study of gap acceptance by left-turning drivers. Transportation Research Record 1899, pp. 71-75.

Dockerty, A. (1966). Accelerations of Queue Leaders from Stop Lines. Traffic Engineering and Control, Vol. 8(3), pp. 150-155.

Dorofeev, Sergey and Grant, Peter (2006). Statistics for Real-Life Sample Surveys, Non-Simple-Random Samples and Weighted Data. Cambridge University Press, Cambridge, UK.

Dravidam U. and Tosunoglu S. (2000). Driver Behavioral factors in Rear-End Collision Avoidance System. Florida Conference on the Recent Advances in Robotics, Florida, USA.

Drew, D. R. (1968). Traffic Flow Theory and Control. McGraw-Hill Inc., New York, N.Y., USA.

Drew, M (1994). Coordinated Adaptive Cruise Control: Design and Simulation Master of Science Dissertation submitted to the Department of Mechanical Engineering at the University of California-Berkeley, CA, USA. 
Dutta, A., Fisher, D., and Noyce, D. (2004). Use of a driving simulator to evaluate and optimize factors affecting understandability of variable message signs. Transportation Research Part F: 7, pp. 209-227, Transportation Research Board, Washington D.C., USA.

Easa, S. M., Dabbour, E., and Ali, M. Z. (2004). Three-Dimensional Model for Stop-Control Intersection Sight Distance. ASCE Journal of Transportation Engineering, Vol. 130(2), pp. 261-270.

Easa, S. M., and Ali, M. Z. (2005). Modified guidelines for left-turn lane geometry at intersections. ASCE Journal for Transportation Engineering. Vol. 131(9), pp. 677-688.

Easa, S. M., Ali, M. Z. and Dabbour, E. (2005). Offsetting opposing left-turn lanes for intersections on horizontal curves. ASCE Journal for Transportation Engineering. Vol. 131(11), pp. 835-842.

Economist Newspaper Group Ltd (ENG) (2001). Beyond Cruise Control. http://www.gavrila.net/Computer Vision/Smart Vehicles/Media Coverage/ec onomist.pdf

Evans, L., and R. W. Rothery (1981). Influence of Vehicle Size and Performance on Intersection Saturation Flow. Proc., 8th International Symposium on Transportation and Traffic Theory, University of Toronto Press, Toronto, Ontario, Canada.

Fambro, D. B., Fitzpatrick, K. and Koppa, R. J. (1997). Determination of Stopping Sight Distances. NCHRP Report 400, Transportation Research Board (TRB), Washington, D.C., USA. 
Fambro, D. B., Koppa, R. J., Picha, D. L. \& Fitzpatrick, K. (1998). Driver Perception-Brake Response in Stopping Sight Distance Situations. Transportation Research Record 1628, Transportation Research Board, National Research Council, Washington, D.C., USA.

Federal Highway Administration (1999). Intersection Collision Warning System.

Publication No. FHWA-RD-99-103. Federal Highway Administration, Washington, D.C., USA.

Federal Highway Administration (2001). Highway Design Handbook for Older Drivers and Pedestrians. U. S. Department of Transportation, Federal Highway Administration (FHWA). Office of Safety - R\&D, McLean, VA, USA. Ferlis, RA (2002). Infrastructure Collision-Avoidance Concept for StraightCrossing-Path Crashes at Signalized Intersections. Transportation Research Record 1800, Transportation Research Board, National Research Council, Washington, D.C., USA.

Fitzpatrick, Kay, Carlson, Paul, Brewer, Marcus A., Wooldridge, Mark D. and Miaou, Shaw-Pin (2003). Design Speed, Operating Speed, and Posted Speed Practices. NCHRP Report 504. Transportation Research Board, National Research Council, Washington, D.C., USA.

Freeman, J. A. and Skapura, D. M. (1991). Neural Networks: algorithms, applications and programming techniques. Addison-Wesley Publishing Company Inc., Indianapolis, IN, USA. 
Fuerstenberg, K. (2005). Intersection Safety - The EC-Project INTERSAFE. Proc.

ITS 2005. 12th World Congress on Intelligent Transport Systems, San Francisco, CA, USA.

Fuerstenberg, K. and J. Chen, J. (2007). New European approach for intersection safety - results of the EC project INTERSAFE. In Proc. International Forum on Advanced Microsystems for Automotive Applications. ISBN-13 978-3-54071324-1. Springer-Verlag, Berlin, Germany.

Glauz, W. D., D. W. Harwood, and A. D. St. John (1980). Projected Vehicle Characteristics Through 1995. In Transportation Research Record 772, Transportation Research Board, National Research Council, Washington, D.C., USA.

Godley, S., Triggs, J., and Fildes, B. (2002). Driving simulator validation for speed research. Accident Analysis and Prevention, Vol. 34(4), pp. 589-600.

Granet, F.; Picado, R.; and Smith, L. (2003). Longitudinal Collision Avoidance. Institute of Transportation Studies at the University of California at Berkeley, CA, USA.

Grewal, M. S. and Andrews, A. P. (1993). Kalman Filtering Theory and Practice. Prentice Hall. New Jersey, NJ, USA.

Groeger, J. A. and Rothegatter, J. A. (1998). Traffic Psychology and Behaviour. Transportation Research Part F 1(1998) pp. 1-9. Transportation Research Board, Washington D.C., USA. 
Harwood, D. W., J. M. Mason, R. E. Brydia, M. T. Pietrucha, and G. L. Gittings (1996). Intersection Sight Distance. NCHRP Report 383. Transportation Research Board, National Research Council, Washington, D.C., USA. Horberry, T., Anderson, J., and Regan, M. A. (2006). The possible safety benefits of enhanced road markings: a driving simulator evaluation. Transportation Research Part F: 9, pp. 77-87, National Research Council, Washington, D.C. USA.

IBEO Automobile Sensor (2009). IBEO laser scanners. Available http://www.ibeo-as.com/english/default.asp

ITS America. (2002). The National ITS Program Plan: A Ten-year Vision. Delivering the Future of Transportation (www.itsa.org/research.html). Jamson, H. (1999). Curve negotiation in the Leeds Driving Simulator: the role of driver experience. Engineering in Psychology and Cognitive Ergonomics, Vol. 3, pp. 351-358.

Jenkins, J., Rilett, L. (2005). Classifying passing maneuvers: a behavioral approach. Transportation Research Record 1937, pp. 14-21, Transportation Research Board, Washington, D.C., USA.

Jocoy, Edward H. and Phoel, Wayne G. (1997). Radar Sensors for Intersection Collision Avoidance. SPIE paper number 2902-32, SPIE Proceedings of Conference on Transportation Sensors and Controls: Collision Avoidance, Traffic Management, and ITS. Vol. 2902, pp. 260-271, Boston, MA, USA. 
Jocoy, Edward H. and Pierwowicz, John (1998). Threat Detection System for Intersection Collision Avoidance. SPIE proceedings Volume 3207, pp. 24-35, Boston, MA, USA.

Johansson, G., and Rumar, K. (1971). Drivers' Brake Reaction Times. Human Factors, 13(1), 23-27.

Kalman, R. E. (1960). A new approach to linear filtering and prediction problems. Journal of Basic Engineering Vol. 82 (1), pp. 35-45.

Kaptein, N., Theeuwes, J., Van Der Horst, R., 1996. Driving simulator validity: Some considerations. Transportation Research Record 1550, pp. 30-36, Transportation Research Board, Washington, D.C., USA.

Kiefer, R., LeBlanc, D., Palmer, M., Salinger, J., Deering, R., and Shulman, M. (1999). Development and Validation of Functional Definitions and Evaluation Procedures for Collision Warning/Avoidance Systems. Report No. DOT HS 808 964. National Highway Traffic Safety Administration. Washington, D.C., USA.

Klee, H., Bauer, C., Radwan, E., and Al-Deek, H. (1999). Preliminary validation of a driving simulator based on forward speed. Transportation Research Record 1689, pp. 33-39, Transportation Research Board, Washington, D.C., USA.

Lee, H. (2002). The validity of driving simulator to measure on-road driving performance of older drivers. Proc. The 24th Conference of Australian Institutes of Transport Research (CAITR 2002), University of New South Wales, Sydney, Australia. 
Lerner, N. D., Huey, R. W., McGee, H. W., and Sullivan, A. (1995). Older driver perception-reaction time for intersection sight distance and object detection. Report No. FHWA-RD-93-168. Federal Highway Administration. Washington, D.C., USA.

Lomax, T., Vadali, S., \& Eisele, W. (2002), Evaluating Intelligent Transportation Systems Impacts: A Framework for Broader Analyses. Report TTI/ITS RCE00/02. Texas Transportation Institute, San Antonio, TX, USA.

Long, G. (2000). Acceleration characteristics of starting vehicles. Transportation Research Record 1737. Transportation Research Board, Washington, D.C., USA.

Mannering, Fred L., Walter P. Kilareski and Scott S. Washburn (2005). Principles of Highway Engineering and Traffic Analysis. Third Edition. John Wiley \& Sons Inc., Hoboken, NJ, USA.

The Mathworks (2009). MATLAB User Guide. The Mathworks http://www.mathworks.com/support/

Maybeck, P. (1979). Stochastic Models Estimation and Control. Academic Press. New York, NY, USA.

McAvoy, D., Schattler, K., and Datta, T. (2007). Driving simulator validation for nighttime construction work zone devices. Proc. The $86^{\text {th }}$ Annual Meeting of the Transportation Research Board, Washington, D.C., USA.

Ministry of Transportation Ontario - MTO (2005). Ontario Road Safety Annual Report. Available from the ministry's website http://www.mto.gov.on.ca/english/safety/orsar/orsar05/ 
Moser, C. A. and Kalton, G. (1971). Survey Methods in Social Investigation. 2nd Edition. Heinemann Educational Books, Portsmouth, NH, USA.

Murray, C.J.L. and A.D. Lopez (1996). The Global Burden of Disease: A Comprehensive Assessment of Mortality and Disability from Diseases, Injuries, and Risk Factors in 1990 and Projected to 2020. Harvard University Press, Cambridge, MA, USA.

Najm, W., Mironer, M., Koziol, J., Wang, J.-S., and Knipling, R. R. (1995). Synthesis Report: Examination of Target Vehicular Crashes and Potential ITS Countermeasures. Report No. DOT HS 808 263, National Highway Traffic Safety Administration, Washington, D.C., USA.

NHTSA (2000). Automotive Collision Avoidance Systems (ACAS) Program. Final Report DOT HS 809 080. National Highway Traffic Safety Administration, Washington, D.C., USA.

NHTSA (2004). Vehicle-Based countermeasures for signal and stop sign violation. Progress Report. DOT HS 809 716. National Highway Traffic Safety Administration, Washington, D.C., USA.

Nilsson, L. (1993). Behavioural research in an advanced driving simulator experiences of the VTI system. Proc. Human Factors and Ergonomics Society $37^{\text {th }}$ Annual Meeting, pp. 612-616.

Norgaard, Magnus (2000). Neural Network Based System Identification Toolbox. Technical Report 00-E-891, Department of Automation, Technical University of Denmark, Denmark. 
Normann, O. K. (1953). Braking Distances of Vehicles from High Speeds. Proc. HRB. Highway Research Board. Vol. 22. pp. 421-436.

Pierwowicz, John (1997). Development of an In-vehicle Intersection Collision Countermeasure. SPIE paper number 2902-34, SPIE Proceedings of Conference on Transportation Sensors and Controls: Collision Avoidance, Traffic Management, and ITS. Volume 2902, pp. 284-292, Boston, MA, USA.

Pierowicz, J., Jocoy, E., Lloyd, M., Bittner, A., and Pirson, B. (2000). Intersection Collision Avoidance Using ITS Countermeasures. Task 9: Final Report. Report No. DOT HS 809 171. National Highway Traffic Safety Administration, Washington, D.C., USA.

Ragland, D. R., Arroyo, S., Shladover, S.E., Misener, J. A. and Chan, C. (2005). Gap acceptance for vehicles turning left across on-coming traffic: Implications for. Report UCB-TSC-RR-2005-TRB4. University of California Berkeley Traffic Safety Center, California, USA.

Rakauskas, M.E., Gugerty, L.J., and Ward, N.J. (2004). Effects of naturalistic cell phone conversations on driving performance. Journal of Safety Research, Vol. 35, pp. 453-464.

Rakha, Hesham, Snare, Matthew, and Dion, Francois (2004). Vehicle dynamics model for estimating maximum light-duty vehicle acceleration levels. Transportation Research Record 1883, pp. 40-49, Transportation Research Board, Washington, D.C., USA.

Rumelhart D. E., Hinton G. E., and William R. J. (1986). Learning internal representations by error propagation. Parallel Distributed Processing, V1: 
Foundations. D. E. Rumelhart and J. L. McClelland, eds., MIT Press, 318362, Cambridge, MA, USA.

Salaani, M. K. and Heydinger, G. J. (2000). Model Validation of the 1997 Jeep Cherokee For the National Advanced Driving Simulator. SAE Paper No. 2000-01-0700, SAE 2000 World Congress, Detroit, MI, USA.

Shirkey, K., Mayhew, G., and Casella, B. (1996). In-vehicle safety advisory and warning system (IVSAWS), Volume I: Executive summary. Report No. FHWA-RD-94-061. Federal Highway Administration, Washington, D.C., USA.

Smart Microwave Sensors (SMS) (2009). Website http://www.smartmicro.de/

Smiley, Alison. Behavioral Adoption, Safety, and Intelligent Transportation Systems. Transportation Research Record 1724. Transportation Research Board, Washington D.C., USA.

Smith, D., Najm, W., and Glassco, R. (2002). Feasibility of driver judgment as a basis for crash avoidance database development. Transportation Research Record 1784. Transportation Research Board, Washington D.C., USA.

Smith, P.; Shah, M.; Da Vitoria Lobo, N. (2000). Monitoring Head/Eye Motion for Driver Alertness with One Camera. Proc., 15th International Conference on Pattern Recognition (ICPR 2000), Barcelona, Spain.

St. John, A. D., and D. R. Kobett (1978). Grade Effects on Traffic Flow Stability and Capacity. NCHRP Report 185. Transportation research Board, National Research Council, Washington, D.C., USA. 
Staplin (1995). Simulator and field measures of driver age differences in left-turn gap judgements. Transportation Research Record 1485, Transportation Research Board, Washington D.C., USA.

STISIM (2009). STISIM User's Guide. Systems Technology Inc., Hawthorne, CA, USA.

Taylor, Sarah J. (2005). Development of a Bayesian Decision Theory Framework to Enhance The Design of Rear-end Collision Warning Systems. Ph.D. thesis, Carleton University, Ottawa, Ontario, Canada.

Tornros, J. (1998). Driving behaviour in a real and a simulated road tunnel-a validation study. Accident Analysis and Prevention, Vol. 30(4), pp. 497-503.

Transport Canada (2001). Vision 2010 Canada's Safety Targets to 2010 (TP 13736 E). Transport Canada, Ottawa, ON, Canada.

Transport Canada (2003). Road Safety in Canada - 2003. (TP 13951 E). Transport Canada, Ottawa, ON, Canada.

Transport Canada (2003). Strategies for Reducing Driver Distraction from InVehicle Telematics Devices: A Discussion Document TP-1413. Transport Canada, Ottawa, ON, Canada.

Transportation Association of Canada (1999). Geometric Design Guide for Canadian Roads. Transportation Association of Canada, Ottawa, ON, Canada.

US Department of Transportation (2000). Intersection Collision Avoidance Using ITS Countermeasures. Final report DOT HS 809 171. Published by Department of Transportation (DOT) and National Highway Traffic Safety 
Administration (NHTSA), Office of Advanced Safety Research. Washington, D.C., USA.

US Department of Transportation (2002). Infrastructure Intersection Collision Avoidance. Intelligent Vehicle Initiative. Federal Highway Administration. Washington, D.C., USA.

US Department of Transportation (2003). Intersection Collision Avoidance Study. Final report published by Department of Transportation (DOT) and Federal Highway Administration (FHWA) Safety Office. Washington, D.C., USA.

VORAD Collision Warning System, produced by EATON (2009). Website $\underline{\text { www.vorad.com }}$

Wang, J., Dixon, K. K., Li, H., and Ogle, J. (2004). Normal acceleration behaviour of passenger vehicles starting from rest at all-way stop-controlled intersections. Transportation Research Record 1883. Transportation Research Board, National Research Council, Washington, D.C., USA.

Watamaniuk, S. N. J. \& Duchon, A. (1992). The human visual system averages speed information. Vision Research, Vol. 24, pp. 47-53.

Wheatley, D. J. \& Hurwitz, J. B. (2001). The use of a multi-modal interface to integrate in-vehicle information presentation. Proc. of the First International Driving Symposium on Human Factors in Driver Assessment, Training, and Vehicle Design, Aspen, CO, USA.

White, Bryon and Eccles, Kimberly A. (2002). Inexpensive, infrastructure-based intersection collision-avoidance system to prevent left-turn crashes with 
opposite-direction traffic. Transportation Research Record 1800, Transportation Research Board, Washington D.C., USA.

Wu, J. D. and Ye, S. H. (2009). Driver identification based on voice signal using continuous wavelet transform and artificial neural network techniques. Expert Systems with Applications, Vol. 36(2), pp. 1061-1069.

Xiao-yun Zhang, X., Jin, X., Qi, W. and Guo, Y. (2008). Vehicle crash accident reconstruction based on the analysis of $3 \mathrm{D}$ deformation of the auto-body. Advances in Engineering Software, Vol. 39(6), pp. 459-465.

Xie, Y., Lord, D. and Zhang, Y. (2007). Predicting motor vehicle collisions using Bayesian neural network models: An empirical analysis. Accident Analysis and Prevention, Vol. 39(5), pp. 922-933.

Yan, Xuedong, Radwan, Essam and Guo, Dahai (2007). Effects of major-road vehicle speed and driver age and gender on left-turn gap acceptance. Accident Analysis and Prevention, Vol. 39(5), pp. 843-852.

Yan, Xuedong, Abdel-Aty, Mohamed, and Radwan, Essam (2008). Validating a driving simulator using surrogate safety measures. Accident Analysis and Prevention, Vol. 40(1), pp. 274-288.

Zador, P. L., Krawchuk, S. A., and Voas, R. B. (2000). Final report - Automotive collision avoidance system (ACAS) program. Report No. DOT HS 808080. National Highway Traffic Safety Administration, Washington, D.C., USA.

Zhang, G., Patuwo, B. E. and Hu, M. Y. (1998). Forecasting with artificial neural networks: The state of the art. International Journal of Forecasting, Vol. 14, pp. 35-62. 
Zheng, N-N.; Tang, S.; Cheng, H.; Li, Q.; Lai, G.; and Wang, F-Y. (2004). Toward intelligent Driver Assistance and Safety Warning System. IEEE Intelligent Systems 9(2), pp. 8-11. 


\section{Glossary of Terms}

$t_{1}$

$t_{2}$

$T_{\text {target }}$

$T_{\text {bullet }}$

$T_{H}$

Range

$V_{H}$

$R_{C A}$

$D_{H S}$

$D_{L S}$

$A_{H}$

$V_{L}$

$A_{L}$

$R T_{H}$

$t$

$\theta_{1}, \theta_{2}, \ldots \theta_{n}$

$d_{1}, d_{2}, \ldots d_{n}$
Driver's perception-reaction time

Travel time required for the target vehicle to make a safe turn

Total time required for target vehicle to make a safe turn

The time required for an approaching vehicle to reach the intersection

Time-headway

the range between the front bumper of the following vehicle and the rear bumper of the lead vehicle

velocity of the following vehicle

the range required for collision avoidance

braking distance for the following vehicle

braking distance for the lead vehicle

average deceleration of the following vehicle

Lead vehicle velocity

average deceleration of the lead vehicle

response time of the following driver

Time interval for detection beam signals

Azimuth angle at different time instances

Range between a detected approaching vehicle and the detection sensor at different time instances

$d_{v 1}, d_{v 2}, \ldots d_{v n} \quad$ Displacement of an approaching vehicle during different time intervals $v_{1}, v_{2}, \ldots v_{n} \quad$ speed of approaching vehicle at different time instances 
$a_{1}, a_{2}, \ldots a_{n} \quad$ Acceleration of approaching vehicle at different time instances

$r$

$w_{f}$

$v_{f}$

$w_{l}$

$S$

$L$
Rate of change in acceleration for an approaching vehicle

offset between the bullet vehicle and the target vehicle

anticipated speed at which the bullet vehicle is anticipated to reach the intersection, lane width (typically $3.00 \mathrm{~m}-3.75 \mathrm{~m}$ )

total distance to be crossed by turning vehicle

length of the turning vehicle 


\section{Appendix "A"}

Simulation Scenario for Left-Turning Vehicles 


\section{METRIC}

-1 Two-lane Rural Road

0 , ROAD , 3.7, 2, 1, 1, 0.15, 0.85, .7, .12, .16, 0, -2, -2, 0, 0, 0, 0, -8, 1.52, -8, 1.52, 0, 0, 0 , C:ISTISIM\DatalTextures\Grass08.Jpg, 25, C:ISTISIM\DatalTextures\Dirt03.Jpg, 12, C:ISTISIM\DatalTextures\Grass08.Jpg, 25

-1 Vehicles travelling on the same direction of the driver

$15, \mathrm{~V}, 18,260,1.85,1,21$

$80, \mathrm{~V}, 20,260,1.85,1,26$

$140, \mathrm{~V}, 19,260,1.85,1, * 18 \sim 35$

$180, \mathrm{~V}, 19,260,1.85,1, * 18 \sim 35$

-1 Control Vehicle for Intersection 01

$0, \mathrm{CV}, 20,0$

282, CV, 0, 0

283, BRK, 45, 1, 40

323, BRK, 70, 2, 0

-1 Start saving dynamic data

330, BSAV, 1, .4975, THROTTLE 01, 1, 2, 6, 27

-1 intersection 01 (distance $350 \mathrm{~m}$ from start), full intersection, left turn only allowed, non signalised

$10, \mathrm{I}, 0,340,3,0,0$

10, SIGN, 13, 280, 0, 1, 0, 0

200, PR, C:ISTISIM \SOUND\Intersection Left.wav, 0, 10

339, PR, C:IZAMBDATAIDriver TraininglEssam Input FileslSTISIM - Sound 14 SEC.WAV, 0, 2

-1 Conflict traffic on opposite direction

220, A, 22, 220, $-1.85, * 18 \sim 35$

230, A, 21, 220, $-1.85, * 18 \sim 35$

245, A, 21, 220, $-1.85, * 18 \sim 35$

270, A, 21, 220, $-1.85, * 18 \sim 35$

310, A, 21, 220, $-1.85, * 18 \sim 35$

$325, \mathrm{~A}, 21,220,-1.85, * 18 \sim 35$

$335, \mathrm{~A}, 21,220,-1.85, * 18 \sim 35$

-1 The driver must turn before the arrival of this vehicle $335, \mathrm{~A}, 17,360,-1.85, * 18 \sim 35$

-1 Other vehicles on opposite direction $335, \mathrm{~A}, 17,385,-1.85, * 18 \sim 35$ 
$335, \mathrm{~A}, 17,400,-1.85, * 18 \sim 35$

$335, \mathrm{~A}, 17,410,-1.85, * 18 \sim 35$

$335, \mathrm{~A}, 17,430,-1.85, * 18 \sim 35$

$335, \mathrm{~A}, 17,440,-1.85, * 18 \sim 35$

$335, \mathrm{~A}, 17,455,-1.85, * 18 \sim 35$

$335, \mathrm{~A}, 16,465,-1.85, * 18 \sim 35$

$335, \mathrm{~A}, 16,480,-1.85, * 18 \sim 35$

$335, \mathrm{~A}, 16,520,-1.85, * 18 \sim 35$

$335, \mathrm{~A}, 15,540,-1.85, * 18 \sim 35$

$335, \mathrm{~A}, 15,555,-1.85, * 18 \sim 35$

-1 End saving dynamic data

369, ESAV

-1 Control Vehicle for Intersection 02

$370, \mathrm{CV}, 20,0$

682, CV, 0, 0

683, BRK, 45, 1, 40

723, BRK, 70, 2, 0

-1 Start saving dynamic data

730, BSAV, 1, .4975, THROTTLE 02, 1, 2, 6, 27

-1 intersection 02 (distance $750 \mathrm{~m}$ from start), full intersection, left turn only allowed, non signalised

$410, \mathrm{I}, 0,340,3,0,0$

410, SIGN, 13, 280, 0, 1, 0, 0

600, PR, C:ISTISIMISOUNDIIntersection Left.wav, 0, 10

739, PR, C:IZAMBDATA\Driver Training\Essam Input Files\STISIM - Sound 16 SEC.WAV, 0, 2

-1 Conflict traffic on opposite direction

620, A, 22, 262, $-1.85, * 18 \sim 35$

$630, \mathrm{~A}, 21,262,-1.85, * 18 \sim 35$

$645, \mathrm{~A}, 21,262,-1.85, * 18 \sim 35$

$670, \mathrm{~A}, 21,262,-1.85, * 18 \sim 35$

710, A, 21, 262, $-1.85, * 18 \sim 35$

$725, \mathrm{~A}, 21,262,-1.85, * 18 \sim 35$

$735, \mathrm{~A}, 21,262,-1.85, * 18 \sim 35$

-1 The driver must turn before the arrival of this vehicle $735, \mathrm{~A}, 18,402,-1.85, * 18 \sim 35$ 
-1 Other vehicles on opposite direction

735, A, 18, 427, -1.85, *18 35

735, A, 18, 442, $-1.85, * 18 \sim 35$

735, A, 18, 452, $-1.85, * 18 \sim 35$

$735, \mathrm{~A}, 18,472,-1.85, * 18 \sim 35$

735, A, 18, 482, $-1.85, * 18 \sim 35$

735, A, 18, 497, -1.85, *18 35

$735, \mathrm{~A}, 17,512,-1.85, * 18 \sim 35$

$735, \mathrm{~A}, 16,522,-1.85, * 18 \sim 35$

$735, \mathrm{~A}, 16,562,-1.85, * 18 \sim 35$

$735, \mathrm{~A}, 16,582,-1.85, * 18 \sim 35$

735, A, 15, 597, $-1.85, * 18 \sim 35$

-1 End saving dynamic data

769, ESAV

-1 Control Vehicle for Intersection 03

770, CV, 19, 0

1182, CV, 0,0

1183, BRK, 45, 1, 40

1225, BRK, 70, 2, 0

-1 Start saving dynamic data

1230, BSAV, 1, .4975, THROTTLE 03, 1, 2, 6, 27

-1 intersection 03 (distance $1250 \mathrm{~m}$ from start), full intersection, left turn only allowed, non signalised

910, I, 0, 340, 3, 0, 0

910, SIGN, 13, 280, 0, 1, 0, 0

1100, PR, C:ISTISIMISOUND Intersection Left.wav, 0, 10

1239, PR, C:IZAMBDATAIDriver TraininglEssam Input FilesISTISIM - Sound\07 SEC.WAV, 0, 2

-1 Conflict traffic on opposite direction

994, A, 22, 362, $-1.85, * 18 \sim 35$

$1004, \mathrm{~A}, 21,362,-1.85, * 18 \sim 35$

$1019, \mathrm{~A}, 21,362,-1.85, * 18 \sim 35$

$1044, \mathrm{~A}, 21,362,-1.85, * 18 \sim 35$

1056, A, 21, 362, $-1.85, * 18 \sim 35$

$1079, \mathrm{~A}, 21,362,-1.85, * 18 \sim 35$

$1098, \mathrm{~A}, 21,362,-1.85, * 18 \sim 35$ 
-1 The driver must turn before the arrival of this vehicle $1109, \mathrm{~A}, 17,522,-1.85, * 18 \sim 35$

-1 Other vehicles on opposite direction $1109, \mathrm{~A}, 17,533,-1.85, * 18 \sim 35$ $1109, \mathrm{~A}, 17,542,-1.85, * 18 \sim 35$ 1109, A, 17, 552, $-1.85, * 18 \sim 35$ $1109, \mathrm{~A}, 17,572,-1.85, * 18 \sim 35$ 1109, A, 16, 582, $-1.85, * 18 \sim 35$ 1109, A, 16, 597, $-1.85, * 18 \sim 35$ $1109, \mathrm{~A}, 16,612,-1.85, * 18 \sim 35$ $1109, \mathrm{~A}, 15,622,-1.85, * 18 \sim 35$ $1109, \mathrm{~A}, 15,662,-1.85, * 18 \sim 35$ $1109, \mathrm{~A}, 15,682,-1.85, * 18 \sim 35$

$1109, \mathrm{~A}, 15,697,-1.85, * 18 \sim 35$

-1 End saving dynamic data

1269, ESAV

-1 Control Vehicle for Intersection 04

$1270, \mathrm{CV}, 21,0$

$1682, \mathrm{CV}, 0,0$

1683, BRK, 45, 1, 40

1723, BRK, 70, 2, 0

-1 Start saving dynamic data

1730, BSAV, 1, .4975, THROTTLE 04, 1, 2, 6, 27

-1 intersection 04 (distance $1750 \mathrm{~m}$ from start), full intersection, left turn only allowed, non signalised 1410, I, 0, 340, 3, 0, 0

1410, SIGN, 13, 280, 0, 1, 0, 0

1600, PR, C:ISTISIM \SOUND Intersection Left.wav, 0, 10

1739, PR, C:IZAMBDATAIDriver TraininglEssam Input Files\STISIM - Sound 11 SEC.WAV, 0, 2

-1 Conflict traffic on opposite direction 1494, A, 22, 409, $-1.85, * 18 \sim 35$

1504, A, 21, 409, $-1.85, * 18 \sim 35$

1519, A, 21, 409, $-1.85, * 18 \sim 35$

1544, A, 21, 409, $-1.85, * 18 \sim 35$

1584, A, 21, 409, $-1.85, * 18 \sim 35$

1599, A, 21, 409, $-1.85, * 18 \sim 35$

1609, A, 21, 409, -1.85, *18 35 
-1 The driver must turn before the arrival of this vehicle $1609, \mathrm{~A}, 18,589,-1.85, * 18 \sim 35$

-1 Other vehicles on opposite direction 1609, A, 18, 611, $-1.85, * 18 \sim 35$ $1609, \mathrm{~A}, 18,629,-1.85, * 18 \sim 35$ 1609, A, 18, 639, -1.85, *18 35 $1609, \mathrm{~A}, 18,653,-1.85, * 18 \sim 35$ 1609, A, 18, 667, $-1.85, * 18 \sim 35$ $1609, \mathrm{~A}, 18,684,-1.85, * 18 \sim 35$ 1609, A, 17, 693, $-1.85, * 18 \sim 35$ $1609, \mathrm{~A}, 17,701,-1.85, * 18 \sim 35$ $1609, \mathrm{~A}, 17,712,-1.85, * 18 \sim 35$ $1609, \mathrm{~A}, 17,729,-1.85, * 18 \sim 35$

$1609, \mathrm{~A}, 17,744,-1.85, * 18 \sim 35$

-1 End saving dynamic data 1769, ESAV

-1 Control Vehicle for Intersection 05

$1770, \mathrm{CV}, 21,0$

2232, CV, 0, 0

2233, BRK, 45, 1,40

2273, BRK, 70, 2, 0

-1 Start saving dynamic data

2280, BSAV, 1, .4975, THROTTLE 05, 1, 2, 6, 27

-1 intersection 05 (distance $2300 \mathrm{~m}$ from start), left-branching intersection, left turn only allowed, non signalised

1960, I, 2, 340, 3, 0, 0

1960, SIGN, 13, 280, 0, 1, 0, 0

2150, PR, C:ISTISIM \SOUND\Intersection Left.wav, 0, 10

2289, PR, C:IZAMBDATAIDriver TraininglEssam Input Files\STISIM - Sound\03 SEC.WAV, 0, 2

-1 Conflict traffic on opposite direction

2044, A, 22, 269, $-1.85, * 18 \sim 35$

2054, A, 21, 269, $-1.85, * 18 \sim 35$

$2069, \mathrm{~A}, 21,269,-1.85, * 18 \sim 35$

2094, A, 21, 269, $-1.85, * 18 \sim 35$

$2114, \mathrm{~A}, 21,269,-1.85, * 18 \sim 35$

$2129, \mathrm{~A}, 21,269,-1.85, * 18 \sim 35$

$2139, \mathrm{~A}, 21,269,-1.85, * 18 \sim 35$ 
-1 The driver must turn before the arrival of this vehicle $2159, \mathrm{~A}, 17,459,-1.85, * 18 \sim 35$

-1 Other vehicles on opposite direction $2159, \mathrm{~A}, 17,474,-1.85, * 18 \sim 35$ 2159, A, 17, 489, $-1.85, * 18 \sim 35$ $2159, \mathrm{~A}, 17,499,-1.85, * 18 \sim 35$ 2159, A, 17, 513, $-1.85, * 18 \sim 35$ $2159, \mathrm{~A}, 16,527,-1.85, * 18 \sim 35$ $2159, \mathrm{~A}, 16,544,-1.85, * 18 \sim 35$ $2159, \mathrm{~A}, 16,553,-1.85, * 18 \sim 35$ 2159, A, 17, 561, $-1.85, * 18 \sim 35$ $2159, \mathrm{~A}, 17,572,-1.85, * 18 \sim 35$

$2159, \mathrm{~A}, 17,589,-1.85, * 18 \sim 35$

2159, A, 17, 604, $-1.85, * 18 \sim 35$

-1 End saving dynamic data

2319, ESAV

-1 Adding curve to the roadway

2400, C, 300, 30, 190, 30, .0025, 1, 4

Changing the road to 4-lane Arterial Road 3000, ROAD, 3.7, 4, 2, 2, 0.15, 0.85, .7, .12, .16, 37, -2, -2, -4, 1.83, -4, 1.83, 0, 1.52, 0, 1.52, 0, 0, 0, C:ISTISIM\DatalTextures\Grass08.Jpg, 25, C:ISTISIM\DatalTextures\Road07.Jpg, 12, C:ISTISIM\DatalTextures\Grass08.Jpg, 25

-1 Vehicles travelling on the same direction of the driver

$3045, \mathrm{~V}, 18,220,5.55,1,21$

$3080, \mathrm{~V}, 17,210,5.55,1,26$

$3140, \mathrm{~V}, 19,210,5.55,1, * 18 \sim 35$

$3180, \mathrm{~V}, 19,230,5.55,1, * 18 \sim 35$

$3380, \mathrm{~V}, 19,230,5.55,1, * 18 \sim 37$

$31080, \mathrm{~V}, 19,185,5.55,1, * 18 \sim 28$

4180, V, 16, 195.64, 5.55, 1, *18 28

4250, V, 20, 195.64, 5.55, 1, *18 28

$4650, \mathrm{~V}, 20,195.64,5.55,1, * 18 \sim 28$

-1 Control Vehicle for Intersection 06 
2320, CV, 19, 0

$3282, \mathrm{CV}, 0,0$

$3283, \mathrm{BRK}, 45,1,40$

3323, BRK, 70, 2, 0

-1 Start saving dynamic data

3330, BSAV, 1, .4975, THROTTLE 06, 1, 2, 6, 27

-1 intersection 06 (distance $3350 \mathrm{~m}$ from start), full intersection, left turn only allowed, non signalised

3010, I, 0, 340, 3, 2, 2

3010, SIGN, 13, 280, 0, 1, 0, 0

3200, PR, C:ISTISIM \SOUND\Intersection Left.wav, 0, 10

3339, PR, C:IZAMBDATAIDriver TraininglEssam Input Files\STISIM - Sound $\backslash 3$ SEC.WAV, 0, 2

-1 Conflict traffic on near lane (left lane of opposite direction)

$3220, \mathrm{~A}, 22,220,-1.85, * 18 \sim 35$

$3230, \mathrm{~A}, 21,220,-1.85, * 18 \sim 35$

$3245, \mathrm{~A}, 21,220,-1.85, * 18 \sim 35$

$3270, \mathrm{~A}, 21,220,-1.85, * 18 \sim 35$

3310, A, $21,220,-1.85, * 18 \sim 35$

$3325, \mathrm{~A}, 21,220,-1.85, * 18 \sim 35$

$3335, \mathrm{~A}, 21,220,-1.85, * 18 \sim 35$

-1 Conflict traffic on far lane (right lane of opposite direction)

$3225, \mathrm{~A}, 22,220,-5.55, * 18 \sim 35$

3240, A, $22,220,-5.55, * 18 \sim 35$

3300, A, 22, 220, $-5.55, * 18 \sim 35$

$3330, \mathrm{~A}, 21,220,-5.55, * 18 \sim 35$

-1 The driver must turn before the arrival of these two vehicles $3335, \mathrm{~A}, 18,380,-1.85, * 18 \sim 35$

$3335, \mathrm{~A}, 18,385,-5.55, * 18 \sim 35$

-1 Other vehicles on near lane (left lane of opposite direction)

$3335, \mathrm{~A}, 18,405,-1.85, * 18 \sim 35$

$3335, \mathrm{~A}, 18,420,-1.85, * 18 \sim 35$

$3335, \mathrm{~A}, 18,430,-1.85, * 18 \sim 35$

$3335, \mathrm{~A}, 19,440,-1.85, * 18 \sim 35$

$3335, \mathrm{~A}, 19,450,-1.85, * 18 \sim 35$

$3335, \mathrm{~A}, 19,465,-1.85, * 18 \sim 35$

$3335, \mathrm{~A}, 18,475,-1.85, * 18 \sim 35$

$3335, \mathrm{~A}, 18,490,-1.85, * 18 \sim 35$

$3335, \mathrm{~A}, 18,520,-1.85, * 18 \sim 35$

$3335, \mathrm{~A}, 19,540,-1.85, * 18 \sim 35$

$3335, \mathrm{~A}, 19,555,-1.85, * 18 \sim 35$

-1 Other vehicles on far lane (right lane of opposite direction) $3335, \mathrm{~A}, 18,400,-5.55, * 18 \sim 35$ 
$3335, \mathrm{~A}, 18,415,-5.55, * 18 \sim 35$

$3335, \mathrm{~A}, 18,425,-5.55, * 18 \sim 35$

$3335, \mathrm{~A}, 18,445,-5.55, * 18 \sim 35$

$3335, \mathrm{~A}, 18,465,-5.55, * 18 \sim 35$

$3335, \mathrm{~A}, 17,485,-5.55, * 18 \sim 35$

$3335, \mathrm{~A}, 17,505,-5.55, * 18 \sim 35$

$3335, \mathrm{~A}, 17,525,-5.55, * 18 \sim 35$

-1 End saving dynamic data

3369, ESAV

-1 Control Vehicle for Intersection 07

$3370, \mathrm{CV}, 18,0$

$3682, \mathrm{CV}, 0,0$

3683, BRK, 45, 1, 40

3723, BRK, 70, 2, 0

-1 Start saving dynamic data

3730, BSAV, 1, .4975, THROTTLE 07, 1, 2, 6, 27

-1 intersection 07 (distance $3750 \mathrm{~m}$ from start), full intersection, left turn only allowed, non signalised

$3410, \mathrm{I}, 0,340,3,2,2$

3410, SIGN, 13, 280, 0, 1, 0, 0

3600, PR, C:ISTISIM \SOUND\Intersection Left.wav, 0, 10

3739, PR, C:IZAMBDATA|Driver TraininglEssam Input Files\STISIM - Sound 15 SEC.WAV, 0, 2

-1 Conflict traffic on near lane (left lane of opposite direction)

$3620, \mathrm{~A}, 22,262,-1.85, * 18 \sim 35$

$3630, \mathrm{~A}, 21,262,-1.85, * 18 \sim 35$

$3645, \mathrm{~A}, 21,262,-1.85, * 18 \sim 35$

$3670, \mathrm{~A}, 21,262,-1.85, * 18 \sim 35$

$3710, \mathrm{~A}, 21,262,-1.85, * 18 \sim 35$

$3725, \mathrm{~A}, 21,262,-1.85, * 18 \sim 35$

$3735, \mathrm{~A}, 21,262,-1.85, * 18 \sim 35$

-1 Conflict traffic on far lane (right lane of opposite direction)

$3625, \mathrm{~A}, 22,262,-5.55, * 18 \sim 35$

3640, A, 22, 262, $-5.55, * 18 \sim 35$

$3700, \mathrm{~A}, 22,262,-5.55, * 18 \sim 35$

3730, A, 21, 262, $-5.55, * 18 \sim 35$

-1 The driver must turn before the arrival of these two vehicles 
$3735, \mathrm{~A}, 19,422,-1.85, * 18 \sim 35$

$3735, \mathrm{~A}, 19,432,-5.55, * 18 \sim 35$

-1 Other vehicles on near lane (left lane of opposite direction)

$3735, \mathrm{~A}, 19,433,-1.85, * 18 \sim 35$

$3735, \mathrm{~A}, 19,442,-1.85, * 18 \sim 35$

$3735, \mathrm{~A}, 19,452,-1.85, * 18 \sim 35$

$3735, \mathrm{~A}, 19,472,-1.85, * 18 \sim 35$

$3735, \mathrm{~A}, 19,482,-1.85, * 18 \sim 35$

$3735, \mathrm{~A}, 19,497,-1.85, * 18 \sim 35$

$3735, \mathrm{~A}, 18,512,-1.85, * 18 \sim 35$

$3735, \mathrm{~A}, 19,522,-1.85, * 18 \sim 35$

$3735, \mathrm{~A}, 18,562,-1.85, * 18 \sim 35$

$3735, \mathrm{~A}, 19,582,-1.85, * 18 \sim 35$

$3735, \mathrm{~A}, 19,597,-1.85, * 18 \sim 35$

-1 Other vehicles on far lane (right lane of opposite direction)

$3735, \mathrm{~A}, 19,440,-5.55, * 18 \sim 35$

$3735, \mathrm{~A}, 19,452,-5.55, * 18 \sim 35$

$3735, \mathrm{~A}, 19,467,-5.55, * 18 \sim 35$

$3735, \mathrm{~A}, 19,487,-5.55, * 18 \sim 35$

$3735, \mathrm{~A}, 19,507,-5.55, * 18 \sim 35$

$3735, \mathrm{~A}, 19,527,-5.55, * 18 \sim 35$

$3735, \mathrm{~A}, 19,547,-5.55, * 18 \sim 35$

$3735, \mathrm{~A}, 19,567,-5.55, * 18 \sim 35$

-1 End saving dynamic data

3769, ESAV

-1 Control Vehicle for Intersection 08

$3770, \mathrm{CV}, 18,0$

4182, CV, 0, 0

4183, BRK, 45, 1, 40

4225, BRK, 70, 2, 0

-1 Start saving dynamic data

4230, BSAV, 1, .4975, THROTTLE 08, 1, 2, 6, 27

-1 intersection 08 (distance $4250 \mathrm{~m}$ from start), full intersection, left turn only allowed, non signalised

3910, I, 0, 340, 3, 2, 2

3910, SIGN, 13, 280, 0, 1, 0, 0

4100, PR, C:ISTISIM\SOUND\Intersection Left.wav, 0, 10 
-1 Conflict traffic on near lane (left lane of opposite direction) $3994, \mathrm{~A}, 22,362,-1.85, * 18 \sim 35$

$4004, \mathrm{~A}, 21,362,-1.85, * 18 \sim 35$

4019, A, 21, 362, $-1.85, * 18 \sim 35$

$4044, \mathrm{~A}, 21,362,-1.85, * 18 \sim 35$

4056, A, 21, 362, $-1.85, * 18 \sim 35$

4079, A, 21, 362, $-1.85, * 18 \sim 35$

$4098, \mathrm{~A}, 21,362,-1.85, * 18 \sim 35$

-1 Conflict traffic on far lane (right lane of opposite direction) 3999, A, 22, 362, $-5.55, * 18 \sim 35$

4014, A, 22, 362, $-5.55, * 18 \sim 35$

$4074, \mathrm{~A}, 22,362,-5.55, * 18 \sim 35$

4096, A, 21, 362, -5.55, *18 35

-1 The driver must turn before the arrival of these two vehicles 4109, A, 19, 522, $-1.85, * 18 \sim 35$

$4109, \mathrm{~A}, 19,532,-5.55, * 18 \sim 35$

-1 Other vehicles on near lane (left lane of opposite direction) 4109, A, 19, 533, $-1.85, * 18 \sim 35$

$4109, \mathrm{~A}, 19,542,-1.85, * 18 \sim 35$

4109, A, 19, 552, $-1.85, * 18 \sim 35$

$4109, \mathrm{~A}, 19,572,-1.85, * 18 \sim 35$

$4109, \mathrm{~A}, 19,582,-1.85, * 18 \sim 35$

4109, A, 19, 597, $-1.85, * 18 \sim 35$

4109, A, 18, 612, $-1.85, * 18 \sim 35$

$4109, \mathrm{~A}, 19,622,-1.85, * 18 \sim 35$

$4109, \mathrm{~A}, 18,662,-1.85, * 18 \sim 35$

$4109, \mathrm{~A}, 19,682,-1.85, * 18 \sim 35$

$4109, \mathrm{~A}, 19,697,-1.85, * 18 \sim 35$

-1 Other vehicles on far lane (right lane of opposite direction) $4109, \mathrm{~A}, 19,541,-5.55, * 18 \sim 35$

$4109, \mathrm{~A}, 19,552,-5.55, * 18 \sim 35$

$4109, \mathrm{~A}, 19,567,-5.55, * 18 \sim 35$

4109, A, 19, 587, $-5.55, * 18 \sim 35$

4109, A, 19, 607, $-5.55, * 18 \sim 35$

4109, A, 19, 627, $-5.55, * 18 \sim 35$

4109, A, 19, 647, $-5.55, * 18 \sim 35$

4109, A, 19, 667, $-5.55, * 18 \sim 35$

-1 End saving dynamic data

4269, ESAV 
-1 Control Vehicle for Intersection 09

$4270, \mathrm{CV}, 21,0$

$4682, \mathrm{CV}, 0,0$

4683, BRK, 45, 1, 40

4723, BRK, 70, 2, 0

-1 Start saving dynamic data

4730, BSAV, 1, .4975, THROTTLE 09, 1, 2, 6, 27

-1 intersection 09 (distance $4750 \mathrm{~m}$ from start), full intersection, left turn only allowed, non signalised

4410, SL, -340, 78, 1, 2, 0, -3, 3, 1, 0

4410, I, 0, 340, 3, 2, 2

4410, SIGN, 13, 280, 0, 1, 0, 0

4600, PR, C:ISTISIMISOUND Intersection Left.wav, 0, 10

4739, PR, C:IZAMBDATAIDriver TraininglEssam Input Files\STISIM - Sound 11 SEC.WAV, 0, 2

-1 Conflict traffic on near lane (left lane of opposite direction)

4494, A, 22, 409, $-1.85, * 18 \sim 35$

4504, A, 21, 409, $-1.85, * 18 \sim 35$

4519, A, $21,409,-1.85, * 18 \sim 35$

4544, A, $21,409,-1.85, * 18 \sim 35$

$4584, \mathrm{~A}, 21,409,-1.85, * 18 \sim 35$

4599, A, 21, 409, $-1.85, * 18 \sim 35$

4609, A, $21,409,-1.85, * 18 \sim 35$

-1 Conflict traffic on far lane (right lane of opposite direction)

4499, A, 22, 409, -5.55, *18 35

4514, A, 22, 409, $-5.55, * 18 \sim 35$

4574, A, 22, 409, $-5.55, * 18 \sim 35$

4604, A, 21, 409, -5.55, *18 35

-1 The driver must turn before the arrival of these two vehicles $4609, \mathrm{~A}, 19,589,-1.85, * 18 \sim 35$

$4609, \mathrm{~A}, 19,599,-5.55, * 18 \sim 35$

-1 Other vehicles on near lane (left lane of opposite direction) 4609, A, 19, 611, $-1.85, * 18 \sim 35$

4609, A, 19, 629, $-1.85, * 18 \sim 35$

$4609, \mathrm{~A}, 19,639,-1.85, * 18 \sim 35$ 
$4609, \mathrm{~A}, 19,653,-1.85, * 18 \sim 35$

4609, A, 19, 667, $-1.85, * 18 \sim 35$

4609, A, 19, 684, $-1.85, * 18 \sim 35$

$4609, \mathrm{~A}, 18,693,-1.85, * 18 \sim 35$

4609, A, 19, 701, $-1.85, * 18 \sim 35$

4609, A, 18, 712, $-1.85, * 18 \sim 35$

$4609, \mathrm{~A}, 19,729,-1.85, * 18 \sim 35$

$4609, \mathrm{~A}, 19,744,-1.85, * 18 \sim 35$

-1 Other vehicles on far lane (right lane of opposite direction) $4609, \mathrm{~A}, 19,612,-5.55, * 18 \sim 35$

$4609, \mathrm{~A}, 19,623,-5.55, * 18 \sim 35$

4609, A, 19, 641, $-5.55, * 18 \sim 35$

4609, A, 19, 654, $-5.55, * 18 \sim 35$

$4609, \mathrm{~A}, 19,667,-5.55, * 18 \sim 35$

$4609, \mathrm{~A}, 19,678,-5.55, * 18 \sim 35$

$4609, \mathrm{~A}, 19,696,-5.55, * 18 \sim 35$

$4609, \mathrm{~A}, 19,714,-5.55, * 18 \sim 35$

-1 End saving dynamic data

4769, ESAV

-1 Control Vehicle for Intersection 10

$4770, \mathrm{CV}, 21,0$

5232, CV, 0, 0

5233, BRK, 45, 1,40

5273, BRK, 70, 2, 0

-1 Start saving dynamic data

5280, BSAV, 1, .4975, THROTTLE 10, 1, 2, 6, 27

-1 intersection 10 (distance $5300 \mathrm{~m}$ from start), full intersection, left turn only allowed, non signalised

4960, I, 0, 340, 3, 2, 2

4960, SIGN, 13, 280, 0, 1, 0, 0

5150, PR, C:ISTISIM \SOUND Intersection Left.wav, 0, 10

5289, PR, C:IZAMBDATAIDriver TraininglEssam Input Files\STISIM - Sound\03 SEC.WAV, 0, 2

-1 Conflict traffic on near lane (left lane of opposite direction)

$5044, \mathrm{~A}, 22,269,-1.85, * 18 \sim 35$

$5054, \mathrm{~A}, 21,269,-1.85, * 18 \sim 35$

$5069, \mathrm{~A}, 21,269,-1.85, * 18 \sim 35$ 
5094, A, 21, 269, -1.85,*18 35

5114, A, 21, 269, -1.85, *18 35

5129, A, 21, 269, -1.85, *18 35

$5139, \mathrm{~A}, 21,269,-1.85, * 18 \sim 35$

-1 Conflict traffic on far lane (right lane of opposite direction)

$5049, \mathrm{~A}, 22,269,-5.55, * 18 \sim 35$

5064, A, 22, 269, $-5.55, * 18 \sim 35$

$5104, \mathrm{~A}, 22,269,-5.55, * 18 \sim 35$

$5134, \mathrm{~A}, 21,269,-5.55, * 18 \sim 35$

-1 The driver must turn before the arrival of these two vehicles $5159, \mathrm{~A}, 19,459,-1.85, * 18 \sim 35$

$5159, \mathrm{~A}, 19,469,-5.55, * 18 \sim 35$

-1 Other vehicles on near lane (left lane of opposite direction)

5159, A, 19, 474, $-1.85, * 18 \sim 35$

5159, A, 19, 489, $-1.85, * 18 \sim 35$

$5159, \mathrm{~A}, 19,499,-1.85, * 18 \sim 35$

$5159, \mathrm{~A}, 19,513,-1.85, * 18 \sim 35$

$5159, \mathrm{~A}, 19,527,-1.85, * 18 \sim 35$

$5159, \mathrm{~A}, 19,544,-1.85, * 18 \sim 35$

$5159, \mathrm{~A}, 18,553,-1.85, * 18 \sim 35$

$5159, \mathrm{~A}, 19,561,-1.85, * 18 \sim 35$

$5159, \mathrm{~A}, 18,572,-1.85, * 18 \sim 35$

$5159, \mathrm{~A}, 19,589,-1.85, * 18 \sim 35$

$5159, \mathrm{~A}, 19,604,-1.85, * 18 \sim 35$

-1 Other vehicles on far lane (right lane of opposite direction)

5159, A, 19, 482, $-5.55, * 18 \sim 35$

$5159, \mathrm{~A}, 19,493,-5.55, * 18 \sim 35$

$5159, \mathrm{~A}, 19,501,-5.55, * 18 \sim 35$

$5159, \mathrm{~A}, 19,514,-5.55, * 18 \sim 35$

$5159, \mathrm{~A}, 19,527,-5.55, * 18 \sim 35$

$5159, \mathrm{~A}, 19,538,-5.55, * 18 \sim 35$

$5159, \mathrm{~A}, 19,556,-5.55, * 18 \sim 35$

$5159, \mathrm{~A}, 19,574,-5.55, * 18 \sim 35$

-1 End saving dynamic data

5319, ESAV

-1 Increasing the number of lanes to 3 lanes in each direction (at distance $5400 \mathrm{~m}$ )

5400, ROAD, 3.7, 6, 3, 0, 0.15, 0.85, .7 . .12, .16, 37, -2, -2, -4, 1.83, -4, 1.83, 0, 1.52, 0, 1.52, 2, 0, 0 , C:ISTISIM\DatalTextures\Grass08.Jpg, 25, C:ISTISIM\DatalTextures\Road07.Jpg, 12,

C:ISTISIM\DatalTextures\Grass08.Jpg, 25, C:ISTISIM\DatalTextures\Grass04.Jpg, 2 
-1 Control Vehicle for Intersection 11

$5320, \mathrm{CV}, 21,0$

$5732, \mathrm{CV}, 0,0$

$5733, \mathrm{BRK}, 45,1,40$

5773, BRK, 70, 2, 0

-1 Start saving dynamic data

5780, BSAV, 1, .4975, THROTTLE 11, 1, 2, 6, 27

-1 intersection 11 (distance $5800 \mathrm{~m}$ from start), full intersection, left turn only allowed, non signalised

5460, SL, -340, 88, 1, 2, 0, -3, 3, 1, 0

$5460, \mathrm{I}, 0,340,3,2,2$

5460, SIGN, 13, 280, 0, 1, 0, 0

5650, PR, C:ISTISIM \SOUND\Intersection Left.wav, 0, 10

5789, PR, C:IZAMBDATAIDriver Training\Essam Input Files\STISIM - Sound\03 SEC.WAV, 0, 2

-1 Conflict traffic on near lane (left lane of opposite direction)

$5544, \mathrm{~A}, 22,269,-2.85, * 18 \sim 35$

$5554, \mathrm{~A}, 21,269,-2.85, * 18 \sim 35$

$5569, \mathrm{~A}, 21,269,-2.85, * 18 \sim 35$

$5594, \mathrm{~A}, 21,269,-2.85, * 18 \sim 35$

5614, A, $21,269,-2.85, * 18 \sim 35$

5629, A, $21,269,-2.85, * 18 \sim 35$

$5639, \mathrm{~A}, 21,269,-2.85, * 18 \sim 35$

-1 Conflict traffic on centre lane of opposite direction

5549, A, 22, 269, $-6.55, * 17 \sim 35$

$5564, \mathrm{~A}, 22,269,-6.55, * 17 \sim 35$

$5604, \mathrm{~A}, 22,269,-6.55, * 17 \sim 35$

$5634, \mathrm{~A}, 21,269,-6.55, * 17 \sim 35$

-1 Conflict traffic on right lane of opposite direction

5554, A, 22, 269, -10.25, *8; 17 35

5566, A, 22, 269, $-10.25, * 8 ; 17 \sim 35$

5611, A, 22, 269, -10.25, *8; 17 35

5627, A, 21, 269, -10.25, *8; 17 35

-1 The driver must turn before the arrival of these two vehicles $5659, \mathrm{~A}, 19,459,-2.85, * 18 \sim 35$

$5659, \mathrm{~A}, 19,469,-6.55, * 18 \sim 35$

-1 Other vehicles on near lane (left lane of opposite direction) 5659, A, 19, 474, -2.85, *18 35

$5659, \mathrm{~A}, 19,489,-2.85, * 18 \sim 35$ 
5659, A, 19, 499, $-2.85, * 18 \sim 35$

$5659, \mathrm{~A}, 19,513,-2.85, * 18 \sim 35$

$5659, \mathrm{~A}, 19,527,-2.85, * 18 \sim 35$

$5659, \mathrm{~A}, 19,544,-2.85, * 18 \sim 35$

$5659, \mathrm{~A}, 18,553,-2.85, * 18 \sim 35$

$5659, \mathrm{~A}, 19,561,-2.85, * 18 \sim 35$

$5659, \mathrm{~A}, 18,572,-2.85, * 18 \sim 35$

$5659, \mathrm{~A}, 19,589,-2.85, * 18 \sim 35$

$5659, \mathrm{~A}, 19,604,-2.85, * 18 \sim 35$

-1 Other vehicles on centre lane of opposite direction

$5659, \mathrm{~A}, 19,482,-6.55, * 18 \sim 35$

5659, A, 19, 493, $-6.55, * 18 \sim 35$

$5659, \mathrm{~A}, 19,501,-6.55, * 18 \sim 35$

$5659, \mathrm{~A}, 19,514,-6.55, * 18 \sim 35$

$5659, \mathrm{~A}, 19,527,-6.55, * 18 \sim 35$

$5659, \mathrm{~A}, 19,538,-6.55, * 18 \sim 35$

$5659, \mathrm{~A}, 19,556,-6.55, * 18 \sim 35$

$5659, \mathrm{~A}, 19,574,-6.55, * 18 \sim 35$

-1 Other vehicles on right lane of opposite direction

$5659, \mathrm{~A}, 19,488,-10.25, * 18 \sim 35$

$5659, \mathrm{~A}, 19,499,-10.25, * 18 \sim 35$

$5659, \mathrm{~A}, 19,516,-10.25, * 18 \sim 35$

-1 End saving dynamic data

5819, ESAV

-1 Control Vehicle for Intersection 12

5820, CV, 21, 0

6232, CV, 0, 0

6233, BRK, 45, 1, 40

6273, BRK, 70, 2, 0

-1 Start saving dynamic data

6280, BSAV, 1, .4975, THROTTLE 12, 1, 2, 6, 27

-1 intersection 12 (distance $6300 \mathrm{~m}$ from start), full intersection, left turn only allowed, non signalised

5960, I, 0, 340, 3, 2, 2

5960, SIGN, 13, 280, 0, 1, 0, 0

6150, PR, C:ISTISIMISOUND\Intersection Left.wav, 0, 10

6289, PR, C:IZAMBDATAIDriver TraininglEssam Input Files\STISIM - Sound109 SEC.WAV, 0, 2 
-1 Conflict traffic on near lane (left lane of opposite direction) $6044, \mathrm{~A}, 22,369,-2.85, * 18 \sim 35$

$6054, \mathrm{~A}, 21,369,-2.85, * 18 \sim 35$

$6069, \mathrm{~A}, 21,369,-2.85, * 18 \sim 35$

6094, A, 21, 369, $-2.85, * 18 \sim 35$

$6114, \mathrm{~A}, 21,369,-2.85, * 18 \sim 35$

$6129, \mathrm{~A}, 21,369,-2.85, * 18 \sim 35$

$6139, \mathrm{~A}, 21,369,-2.85, * 18 \sim 35$

-1 Conflict traffic on centre lane of opposite direction $6049, \mathrm{~A}, 22,369,-6.55, * 17 \sim 35$

6064, A, 22, 369, $-6.55, * 17 \sim 35$

6104, A, 22, 369, -6.55, *17 35

$6134, \mathrm{~A}, 21,369,-6.55, * 17 \sim 35$

-1 Conflict traffic on right lane of opposite direction

6054, A, 22, 369, $-10.25, * 8 ; 17 \sim 35$

6066, A, 22, 369, -10.25, *8; 17 35

6111, A, 22, 369, -10.25, *8; 17 35

6127, A, 21, 369, -10.25, *8; 17 35

-1 The driver must turn before the arrival of these two vehicles 6159, A, 19, 559, -2.85, *18 35

$6159, \mathrm{~A}, 19,569,-6.55, * 18 \sim 35$

-1 Other vehicles on near lane (left lane of opposite direction) $6159, \mathrm{~A}, 19,574,-2.85, * 18 \sim 35$

6159, A, 19, 589, $-2.85, * 18 \sim 35$

$6159, \mathrm{~A}, 19,599,-2.85, * 18 \sim 35$

$6159, \mathrm{~A}, 19,613,-2.85, * 18 \sim 35$

$6159, \mathrm{~A}, 19,627,-2.85, * 18 \sim 35$

$6159, \mathrm{~A}, 19,644,-2.85, * 18 \sim 35$

$6159, \mathrm{~A}, 18,653,-2.85, * 18 \sim 35$

$6159, \mathrm{~A}, 19,661,-2.85, * 18 \sim 35$

$6159, \mathrm{~A}, 18,672,-2.85, * 18 \sim 35$

$6159, \mathrm{~A}, 19,689,-2.85, * 18 \sim 35$

$6159, \mathrm{~A}, 19,704,-2.85, * 18 \sim 35$

-1 Other vehicles on centre lane of opposite direction $6159, \mathrm{~A}, 19,582,-6.55, * 18 \sim 35$

6159, A, 19, 593, $-6.55, * 18 \sim 35$

$6159, \mathrm{~A}, 19,601,-6.55, * 18 \sim 35$

$6159, \mathrm{~A}, 19,614,-6.55, * 18 \sim 35$

$6159, \mathrm{~A}, 19,627,-6.55, * 18 \sim 35$

$6159, \mathrm{~A}, 19,638,-6.55, * 18 \sim 35$

6159, A, 19, 656, $-6.55, * 18 \sim 35$

$6159, \mathrm{~A}, 19,674,-6.55, * 18 \sim 35$ 
-1 End saving dynamic data

6319, ESAV

-1 Control Vehicle for Intersection 13

$6320, \mathrm{CV}, 21,0$

$6732, \mathrm{CV}, 0,0$

6733, BRK, 45, 1, 40

6773, BRK, 70, 2, 0

-1 Start saving dynamic data

6780, BSAV, 1, .4975, THROTTLE 13, 1, 2, 6, 27

-1 intersection 13 (distance $6800 \mathrm{~m}$ from start), full intersection, left turn only allowed, non signalised

6460, SL, $-340,85,1,2,0,-3,3,1,0$

6460, I, 0, 340, 3, 2, 2

6460, SIGN, 13, 280, 0, 1, 0, 0

6650, PR, C:ISTISIM \SOUND\Intersection Left.wav, 0, 10

6789, PR, C:IZAMBDATAIDriver TraininglEssam Input Files\STISIM - Sound\07 SEC.WAV, 0, 2

-1 Conflict traffic on near lane (left lane of opposite direction)

$6544, \mathrm{~A}, 22,329,-2.85, * 18 \sim 35$

6554, A, 21, 329, $-2.85, * 18 \sim 35$

$6569, \mathrm{~A}, 21,329,-2.85, * 18 \sim 35$

6594, A, 21, 329, $-2.85, * 18 \sim 35$

$6614, \mathrm{~A}, 21,329,-2.85, * 18 \sim 35$

$6629, \mathrm{~A}, 21,329,-2.85, * 18 \sim 35$

$6639, \mathrm{~A}, 21,329,-2.85, * 18 \sim 35$

-1 Conflict traffic on centre lane of opposite direction

6549, A, 22, 329, $-6.55, * 17 \sim 35$

6564, A, 22, 329, $-6.55, * 17 \sim 35$

6604, A, 22, 329, $-6.55, * 17 \sim 35$

6634, A, 21, 329, $-6.55, * 17 \sim 35$

-1 Conflict traffic on right lane of opposite direction

6554, A, 22, 329, -10.25, *8; 17 35

6566, A, 22, 329, $-10.25, * 8 ; 17 \sim 35$

6611, A, 22, 329, -10.25, *8; 17 35

6627, A, 21, 329, -10.25, *8; 17 35

-1 The driver must turn before the arrival of these three vehicles 
$6659, \mathrm{~A}, 19,519,-2.85, * 18 \sim 35$

$6659, \mathrm{~A}, 19,529,-6.55, * 18 \sim 35$

$6659, \mathrm{~A}, 19,534,-10.25, * 18 \sim 35$

-1 Other vehicles on near lane (left lane of opposite direction) $6659, \mathrm{~A}, 19,534,-2.85, * 18 \sim 35$

$6659, \mathrm{~A}, 19,549,-2.85, * 18 \sim 35$

$6659, \mathrm{~A}, 19,559,-2.85, * 18 \sim 35$

$6659, \mathrm{~A}, 19,573,-2.85, * 18 \sim 35$

$6659, \mathrm{~A}, 19,587,-2.85, * 18 \sim 35$

$6659, \mathrm{~A}, 19,604,-2.85, * 18 \sim 35$

$6659, \mathrm{~A}, 18,613,-2.85, * 18 \sim 35$

$6659, \mathrm{~A}, 19,621,-2.85, * 18 \sim 35$

$6659, \mathrm{~A}, 18,632,-2.85, * 18 \sim 35$

$6659, \mathrm{~A}, 19,649,-2.85, * 18 \sim 35$

$6659, \mathrm{~A}, 19,664,-2.85, * 18 \sim 35$

-1 Other vehicles on centre lane of opposite direction $6659, \mathrm{~A}, 19,542,-6.55, * 18 \sim 35$

$6659, \mathrm{~A}, 19,553,-6.55, * 18 \sim 35$

$6659, \mathrm{~A}, 19,561,-6.55, * 18 \sim 35$

$6659, \mathrm{~A}, 19,574,-6.55, * 18 \sim 35$

$6659, \mathrm{~A}, 19,587,-6.55, * 18 \sim 35$

$6659, \mathrm{~A}, 19,598,-6.55, * 18 \sim 35$

$6659, \mathrm{~A}, 19,616,-6.55, * 18 \sim 35$

$6659, \mathrm{~A}, 19,634,-6.55, * 18 \sim 35$

-1 End saving dynamic data

6819, ESAV

-1 Vertical curve at distance $7350 \mathrm{~m}$ from start

7350, VC, 200, .02

-1 Control Vehicle for Intersection 14

6820, CV, 21, 0

7432, CV, 0, 0

7433, BRK, 45, 1, 40

7473, BRK, 70, 2, 0

-1 Start saving dynamic data

7480, BSAV , 1, .4975, THROTTLE 14, 1, 2, 6, 27 
-1 intersection 14 (distance 7500 m from start), full intersection, left turn only allowed, non signalised

7160, SL, -340, 94, 1, 2, 0, -3, 3, 1, 0

7160, I, 0, 340, 3, 2, 2

7160, SIGN, 13, 280, 0, 1, 0, 0

7350, PR, C:ISTISIMISOUND Intersection Left.wav, 0, 10

7489, PR, C:IZAMBDATAIDriver TraininglEssam Input Files\STISIM - Sound 16 SEC.WAV, 0, 2

-1 Conflict traffic on near lane (left lane of opposite direction)

7244, A, 22, 489, $-2.85, * 18 \sim 35$

$7254, \mathrm{~A}, 21,489,-2.85, * 18 \sim 35$

$7269, \mathrm{~A}, 21,489,-2.85, * 18 \sim 35$

$7294, \mathrm{~A}, 21,489,-2.85, * 18 \sim 35$

7314, A, 21, 489, $-2.85, * 18 \sim 35$

7329, A, 21, 489, $-2.85, * 18 \sim 35$

7339, A, 21, 489, $-2.85, * 18 \sim 35$

-1 Conflict traffic on centre lane of opposite direction

7249, A, 22, 489, $-6.55, * 17 \sim 35$

7264, A, 22, 489, $-6.55, * 17 \sim 35$

7304, A, 22, 489, $-6.55, * 17 \sim 35$

$7334, \mathrm{~A}, 21,489,-6.55, * 17 \sim 35$

-1 Conflict traffic on right lane of opposite direction

7254, A, 22, 489, -10.25, *8; 17 35

7266, A, 22, 489, $-10.25, * 8 ; 17 \sim 35$

7311, A, 22, 489, $-10.25, * 8 ; 17 \sim 35$

7327, A, 21, 489, $-10.25, * 8 ; 17 \sim 35$

-1 The driver must turn before the arrival of these three vehicles

$7359, \mathrm{~A}, 19,679,-2.85, * 18 \sim 35$

$7359, \mathrm{~A}, 19,689,-6.55, * 18 \sim 35$

$7359, \mathrm{~A}, 19,694,-10.25, * 18 \sim 35$

-1 Other vehicles on near lane (left lane of opposite direction)

$7359, \mathrm{~A}, 19,694,-2.85, * 18 \sim 35$

$7359, \mathrm{~A}, 19,709,-2.85, * 18 \sim 35$

7359, A, 19, 719, $-2.85, * 18 \sim 35$

7359, A, 19, 733, $-2.85, * 18 \sim 35$

$7359, \mathrm{~A}, 19,747,-2.85, * 18 \sim 35$

$7359, \mathrm{~A}, 19,764,-2.85, * 18 \sim 35$

$7359, \mathrm{~A}, 18,773,-2.85, * 18 \sim 35$

$7359, \mathrm{~A}, 19,781,-2.85, * 18 \sim 35$

$7359, \mathrm{~A}, 18,792,-2.85, * 18 \sim 35$ 
7359, A, 19, 809, $-2.85, * 18 \sim 35$

$7359, \mathrm{~A}, 19,824,-2.85, * 18 \sim 35$

-1 Other vehicles on centre lane of opposite direction

7359, A, 19, 702, $-6.55, * 18 \sim 35$

7359, A, 19, 713, $-6.55, * 18 \sim 35$

7359, A, 19, 721, $-6.55, * 18 \sim 35$

$7359, \mathrm{~A}, 19,734,-6.55, * 18 \sim 35$

7359, A, 19, 747, $-6.55, * 18 \sim 35$

$7359, \mathrm{~A}, 19,758,-6.55, * 18 \sim 35$

7359, A, 19, 776, $-6.55, * 18 \sim 35$

7359, A, 19, 794, $-6.55, * 18 \sim 35$

-1 End saving dynamic data

7519, ESAV

-1 Control Vehicle for Intersection 15

7520, CV, 21, 0

7932, CV, 0, 0

7933, BRK, 45, 1, 40

7973, BRK, 70, 2, 0

-1 Start saving dynamic data

7980, BSAV, 1, .4975, THROTTLE 15, 1, 2, 6, 27

-1 intersection 15 (distance $8000 \mathrm{~m}$ from start), full intersection, left turn only allowed, signalised

7660, SL, -340, 86, 2, 2, 0, -3, 3, 1, 0

$7660, \mathrm{I}, 0,340,3,2,2$

7660, SIGN, 13, 280, 0, 1, 0, 0

7850, PR, C:ISTISIMISOUND Intersection Left.wav, 0, 10

7989, PR, C:IZAMBDATAIDriver TraininglEssam Input Files\STISIM - Sound 10 SEC.WAV, 0, 2

-1 Conflict traffic on near lane (left lane of opposite direction)

7744, A, 22, 389, $-2.85, * 18 \sim 35$

7754, A, 21, 389, $-2.85, * 18 \sim 35$

7769, A, 21, 389, $-2.85, * 18 \sim 35$

7794, A, 21, 389, $-2.85, * 18 \sim 35$

7814, A, 21, 389, $-2.85, * 18 \sim 35$

7829, A, 21, 389, $-2.85, * 18 \sim 35$

7839, A, 21, 389, $-2.85, * 18 \sim 35$

-1 Conflict traffic on centre lane of opposite direction 
7749, A, 22, 389, $-6.55, * 17 \sim 35$

$7764, \mathrm{~A}, 22,389,-6.55, * 17 \sim 35$

$7804, \mathrm{~A}, 22,389,-6.55, * 17 \sim 35$

7834, A, 21, 389, -6.55, *17 35

-1 Conflict traffic on right lane of opposite direction

7754, A, 22, 389, -10.25, *8; 17 35

7766, A, 22, 389, -10.25, *8; 17 35

7811, A, 22, 389, $-10.25, * 8 ; 17 \sim 35$

7827, A, 21, 389, -10.25, *8; 17 35

-1 The driver must turn before the arrival of these three vehicles $7859, \mathrm{~A}, 19,599,-2.85, * 18 \sim 35$

$7859, \mathrm{~A}, 19,609,-6.55, * 18 \sim 35$

$7859, \mathrm{~A}, 19,614,-10.25, * 18 \sim 35$

-1 Other vehicles on near lane (left lane of opposite direction)

7859, A, 19, 624, $-2.85, * 18 \sim 35$

7859, A, 19, 639, $-2.85, * 18 \sim 35$

7859, A, 19, 659, $-2.85, * 18 \sim 35$

7859, A, 19, 673, $-2.85, * 18 \sim 35$

7859, A, 19, 687, $-2.85, * 18 \sim 35$

$7859, \mathrm{~A}, 19,694,-2.85, * 18 \sim 35$

7859, A, 18, 713, $-2.85, * 18 \sim 35$

$7859, \mathrm{~A}, 19,731,-2.85, * 18 \sim 35$

7859, A, 18, 752, $-2.85, * 18 \sim 35$

7859, A, 19, 769, $-2.85, * 18 \sim 35$

7859, A, 19, 784, $-2.85, * 18 \sim 35$

-1 Other vehicles on centre lane of opposite direction 7859, A, 19, 622, $-6.55, * 18 \sim 35$

7859, A, 19, 633, $-6.55, * 18 \sim 35$

7859, A, 19, 641, $-6.55, * 18 \sim 35$

$7859, \mathrm{~A}, 19,654,-6.55, * 18 \sim 35$

7859, A, 19, 667, $-6.55, * 18 \sim 35$

$7859, \mathrm{~A}, 19,678,-6.55, * 18 \sim 35$

7859, A, 19, 686, $-6.55, * 18 \sim 35$

7859, A, 19, 694, $-6.55, * 18 \sim 35$

-1 End saving dynamic data

8019, ESAV

-1 Adding curve to the roadway

7800, C, 300, 30, 90, 30, -.0025, 1, -4 
-1 Decreasing the number of lanes to 2 lanes in each direction with median and traffic signal (at distance $8200 \mathrm{~m}$ )

8300, ROAD, 3.7, 4, 2, 0, 0.15, 0.85, .7, .12, .16, 42, -2, -2, -4, 1.83, -4, 1.83, 0, 1.52, 0, 1.52, 2, 0, 0 , C:ISTISIM\DatalTextureslGrass08.Jpg, 25, C:ISTISIM\DatalTextures\Road07.Jpg, 12, C:ISTISIM $\backslash$ DatalTextures\Grass08.Jpg, 25, C:ISTISIM\DatalTextures\Grass04.Jpg, 2

-1 Control Vehicle for Intersection 16

$8020, \mathrm{CV}, 21,0$

$8432, \mathrm{CV}, 0,0$

8433, BRK, 45, 1,40

8473 , BRK, 55, 2, 0

-1 Start saving dynamic data

8480, BSAV $, 1, .4975$, THROTTLE $11,1,2,6,27$

-1 intersection 16 (distance $8500 \mathrm{~m}$ from start), full intersection, left turn only allowed, signalised

8160, I, 0, 340, 3, 2, 2

8160, SIGN, 13, 280, 0, 1, 0, 0

8350, PR, C:ISTISIM \SOUND\Intersection Left.wav, 0, 10

8489, PR, C:IZAMBDATAIDriver TrainingIEssam Input FilesISTISIM - Sound 03 SEC.WAV, 0, 2

-1 Conflict traffic on near lane (left lane of opposite direction)

8244, A, $22,269,-2.85, * 18 \sim 35$

8254, A, $21,269,-2.85, * 18 \sim 35$

$8269, \mathrm{~A}, 21,269,-2.85, * 18 \sim 35$

8294, A, $21,269,-2.85, * 18 \sim 35$

$8314, \mathrm{~A}, 21,269,-2.85, * 18 \sim 35$

$8329, \mathrm{~A}, 21,269,-2.85, * 18 \sim 35$

$8339, \mathrm{~A}, 21,269,-2.85, * 18 \sim 35$

-1 Conflict traffic on far lane of opposite direction

8249, A, 22, 269, $-6.55, * 17 \sim 35$

8264, A, 22, 269, $-6.55, * 17 \sim 35$

8304, A, 22, 269, $-6.55, * 17 \sim 35$

8334, A, 21, 269, $-6.55, * 17 \sim 35$

-1 The driver must turn before the arrival of these two vehicles $8359, \mathrm{~A}, 19,459,-2.85, * 18 \sim 35$

$8359, \mathrm{~A}, 19,469,-6.55, * 18 \sim 35$

-1 Other vehicles on near lane (left lane of opposite direction) 
$8359, \mathrm{~A}, 19,474,-2.85, * 18 \sim 35$

8359, A, 19, 489, $-2.85, * 18 \sim 35$

$8359, \mathrm{~A}, 19,499,-2.85, * 18 \sim 35$

$8359, \mathrm{~A}, 19,513,-2.85, * 18 \sim 35$

$8359, \mathrm{~A}, 19,527,-2.85, * 18 \sim 35$

$8359, \mathrm{~A}, 19,544,-2.85, * 18 \sim 35$

$8359, \mathrm{~A}, 18,553,-2.85, * 18 \sim 35$

$8359, \mathrm{~A}, 19,561,-2.85, * 18 \sim 35$

8359, A, 18, 572, $-2.85, * 18 \sim 35$

$8359, \mathrm{~A}, 19,589,-2.85, * 18 \sim 35$

$8359, \mathrm{~A}, 19,604,-2.85, * 18 \sim 35$

-1 Other vehicles on far lane of opposite direction 8359, A, 19, 482, $-6.55, * 18 \sim 35$

8359, A, 19, 493, $-6.55, * 18 \sim 35$

$8359, \mathrm{~A}, 19,501,-6.55, * 18 \sim 35$

8359, A, 19, 514, $-6.55, * 18 \sim 35$

8359, A, 19, 527, $-6.55, * 18 \sim 35$

8359, A, 19, 538, $-6.55, * 18 \sim 35$

$8359, \mathrm{~A}, 19,556,-6.55, * 18 \sim 35$

$8359, \mathrm{~A}, 19,574,-6.55, * 18 \sim 35$

-1 End saving dynamic data

8519, ESAV

-1 Control Vehicle for Intersection 17

$8520, \mathrm{CV}, 18,0$

8932, CV, 0, 0

8933, BRK, 45, 1, 40

8973, BRK, 55, 2, 0

-1 Start saving dynamic data

8980, BSAV, 1, .4975, THROTTLE 07, 1, 2, 6, 27

-1 intersection 17 (distance $9000 \mathrm{~m}$ from start), full intersection, left turn only allowed, non signalised

$8660, \mathrm{I}, 0,340,3,2,2$

8660, SIGN, 13, 280, 0, 1, 0, 0

8850, PR, C:ISTISIMISOUND Intersection Left.wav, 0, 10

8986, PR, C:IZAMBDATAIDriver TraininglEssam Input Files\STISIM - Sound 15 SEC.WAV, 0, 2

-1 Conflict traffic on near lane (left lane of opposite direction) 
8870, A, 22, 262, $-1.85, * 18 \sim 35$

8880, A, $21,262,-1.85, * 18 \sim 35$

8895, A, $21,262,-1.85, * 18 \sim 35$

8920, A, 21, 262, $-1.85, * 18 \sim 35$

8960, A, $21,262,-1.85, * 18 \sim 35$

$8975, \mathrm{~A}, 21,262,-1.85, * 18 \sim 35$

$8985, \mathrm{~A}, 21,262,-1.85, * 18 \sim 35$

-1 Conflict traffic on far lane (right lane of opposite direction) $8875, \mathrm{~A}, 22,262,-5.55, * 18 \sim 35$

$8890, \mathrm{~A}, 22,262,-5.55, * 18 \sim 35$

$8950, \mathrm{~A}, 22,262,-5.55, * 18 \sim 35$

8980, A, $21,262,-5.55, * 18 \sim 35$

-1 The driver must turn before the arrival of these two vehicles $8985, \mathrm{~A}, 19,402,-1.85, * 18 \sim 35$

$8985, \mathrm{~A}, 19,412,-5.55, * 18 \sim 35$

-1 Other vehicles on near lane (left lane of opposite direction) $8985, \mathrm{~A}, 19,427,-1.85, * 18 \sim 35$

$8985, \mathrm{~A}, 19,442,-1.85, * 18 \sim 35$

$8985, \mathrm{~A}, 19,452,-1.85, * 18 \sim 35$

$8985, \mathrm{~A}, 19,472,-1.85, * 18 \sim 35$

$8985, \mathrm{~A}, 19,482,-1.85, * 18 \sim 35$

$8985, \mathrm{~A}, 19,497,-1.85, * 18 \sim 35$

$8985, \mathrm{~A}, 18,512,-1.85, * 18 \sim 35$

$8985, \mathrm{~A}, 19,522,-1.85, * 18 \sim 35$

$8985, \mathrm{~A}, 18,562,-1.85, * 18 \sim 35$

$8985, \mathrm{~A}, 19,582,-1.85, * 18 \sim 35$

$8985, \mathrm{~A}, 19,597,-1.85, * 18 \sim 35$

-1 Other vehicles on far lane (right lane of opposite direction) 8985, A, 19, 427, $-5.55, * 18 \sim 35$

$8985, \mathrm{~A}, 19,447,-5.55, * 18 \sim 35$

$8985, \mathrm{~A}, 19,467,-5.55, * 18 \sim 35$

$8985, \mathrm{~A}, 19,487,-5.55, * 18 \sim 35$

$8985, \mathrm{~A}, 19,507,-5.55, * 18 \sim 35$

$8985, \mathrm{~A}, 19,527,-5.55, * 18 \sim 35$

8985, A, 19, 547, -5.55, *18 35

$8985, \mathrm{~A}, 19,567,-5.55, * 18 \sim 35$

-1 End saving dynamic data

9019, ESAV

-1 Control Vehicle for Intersection 18 
9020, CV, 21, 0

9232, CV, 0, 0

9233, BRK, 45, 1, 40

9273, BRK, 55, 2, 0

-1 Start saving dynamic data

9280, BSAV, 1, .4975, THROTTLE 12, 1, 2, 6, 27

-1 intersection 18 (distance $9300 \mathrm{~m}$ from start), full intersection, left turn only allowed, non signalised

$8960, \mathrm{I}, 0,340,3,2,2$

8960, SIGN, 13, 280, 0, 1, 0, 0

9150, PR, C:ISTISIMISOUND\Intersection Left.wav, 0, 10

9289, PR, C:IZAMBDATAIDriver TrainingIEssam Input FilesISTISIM - Sound\09 SEC.WAV, 0, 2

-1 Conflict traffic on near lane (left lane of opposite direction)

9044, A, $22,369,-2.85, * 18 \sim 35$

9054, A, 21, 369, $-2.85, * 18 \sim 35$

$9069, \mathrm{~A}, 21,369,-2.85, * 18 \sim 35$

9094, A, 21, 369, $-2.85, * 18 \sim 35$

9114, A, 21, 369, $-2.85, * 18 \sim 35$

9129, A, 21, 369, $-2.85, * 18 \sim 35$

$9139, \mathrm{~A}, 21,369,-2.85, * 18 \sim 35$

-1 Conflict traffic on far lane of opposite direction

9049, A, 22, 369, $-6.55, * 17 \sim 35$

9064, A, 22, 369, $-6.55, * 17 \sim 35$

9104, A, 22, 369, $-6.55, * 17 \sim 35$

9134, A, 21, 369, $-6.55, * 17 \sim 35$

-1 The driver must turn before the arrival of these two vehicles

$9159, \mathrm{~A}, 19,559,-2.85, * 18 \sim 35$

$9159, \mathrm{~A}, 19,569,-6.55, * 18 \sim 35$

-1 Other vehicles on near lane (left lane of opposite direction)

9159, A, 19, 574, $-2.85, * 18 \sim 35$

$9159, \mathrm{~A}, 19,589,-2.85, * 18 \sim 35$

9159, A, 19, 599, $-2.85, * 18 \sim 35$

9159, A, 19, 613, $-2.85, * 18 \sim 35$

$9159, \mathrm{~A}, 19,627,-2.85, * 18 \sim 35$

$9159, \mathrm{~A}, 19,644,-2.85, * 18 \sim 35$

$9159, \mathrm{~A}, 18,653,-2.85, * 18 \sim 35$

9159, A, 19, 661, $-2.85, * 18 \sim 35$

$9159, \mathrm{~A}, 18,672,-2.85, * 18 \sim 35$ 
9159, A, 19, 689, $-2.85, * 18 \sim 35$

9159, A, 19, 704, $-2.85, * 18 \sim 35$

-1 Other vehicles on far lane of opposite direction

9159, A, 19, 582, $-6.55, * 18 \sim 35$

9159, A, 19, 593, $-6.55, * 18 \sim 35$

9159, A, 19, 601, $-6.55, * 18 \sim 35$

$9159, \mathrm{~A}, 19,614,-6.55, * 18 \sim 35$

$9159, \mathrm{~A}, 19,627,-6.55, * 18 \sim 35$

9159, A, 19, 638, $-6.55, * 18 \sim 35$

$9159, \mathrm{~A}, 19,656,-6.55, * 18 \sim 35$

9159, A, 19, 674, $-6.55, * 18 \sim 35$

-1 End saving dynamic data

9319, ESAV

-1 Control Vehicle for Intersection 19

9320, CV, 21, 0

9682, CV, 0, 0

9683, BRK, 45, 1, 40

9723, BRK, 55, 2, 0

-1 Start saving dynamic data

9730, BSAV, 1, .4975, THROTTLE 04, 1, 2, 6, 27

-1 intersection 19 (distance $9750 \mathrm{~m}$ from start), full intersection, left turn only allowed, non signalised

9410, I, 2, 340, 3, 0, 0

9410, SIGN, 13, 280, 0, 1, 0, 0

9600, PR, C:ISTISIMISOUND Intersection Left.wav, 0, 10

9736, PR, C:IZAMBDATAIDriver TraininglEssam Input Files\STISIM - Sound 11 SEC.WAV, 0, 2

-1 Conflict traffic on near lane (left lane of opposite direction)

9494, A, 22, 409, $-1.85, * 18 \sim 35$

9504, A, 21, 409, $-1.85, * 18 \sim 35$

9519, A, 21, 409, $-1.85, * 18 \sim 35$

9544, A, 21, 409, $-1.85, * 18 \sim 35$

9584, A, 21, 409, $-1.85, * 18 \sim 35$

9599, A, 21, 409, $-1.85, * 18 \sim 35$

9609, A, 21, 409, -1.85, *18 35

-1 Conflict traffic on far lane (right lane of opposite direction) 
9499, A, 22, 409, $-5.55, * 18 \sim 35$

9514, A, 22, 409, $-5.55, * 18 \sim 35$

9574, A, 22, 409, $-5.55, * 18 \sim 35$

9604, A, 21, 409, -5.55, *18 35

-1 The driver must turn before the arrival of these two vehicles $9609, \mathrm{~A}, 19,589,-1.85, * 18 \sim 35$

9609, A, 19, 599, -5.55, *18 35

-1 Other vehicles on near lane (left lane of opposite direction) 9609, A, 19, 611, $-1.85, * 18 \sim 35$

$9609, \mathrm{~A}, 19,629,-1.85, * 18 \sim 35$

$9609, \mathrm{~A}, 19,639,-1.85, * 18 \sim 35$

$9609, \mathrm{~A}, 19,653,-1.85, * 18 \sim 35$

9609, A, 19, 667, $-1.85, * 18 \sim 35$

$9609, \mathrm{~A}, 19,684,-1.85, * 18 \sim 35$

9609, A, 18, 693, $-1.85, * 18 \sim 35$

9609, A, 19, 701, $-1.85, * 18 \sim 35$

$9609, \mathrm{~A}, 18,712,-1.85, * 18 \sim 35$

$9609, \mathrm{~A}, 19,729,-1.85, * 18 \sim 35$

9609, A, 19, 744, $-1.85, * 18 \sim 35$

-1 Other vehicles on far lane (right lane of opposite direction) 9609, A, 19, 612, $-5.55, * 18 \sim 35$

$9609, \mathrm{~A}, 19,623,-5.55, * 18 \sim 35$

$9609, \mathrm{~A}, 19,641,-5.55, * 18 \sim 35$

$9609, \mathrm{~A}, 19,654,-5.55, * 18 \sim 35$

$9609, \mathrm{~A}, 19,667,-5.55, * 18 \sim 35$

$9609, \mathrm{~A}, 19,678,-5.55, * 18 \sim 35$

9609, A, 19, 696, $-5.55, * 18 \sim 35$

$9609, \mathrm{~A}, 19,714,-5.55, * 18 \sim 35$

-1 End saving dynamic data

9769, ESAV

-1 Control Vehicle for Intersection 20

9770, CV, 18, 0

10282, CV, 0, 0

10283, BRK, 45, 1, 40

10323, BRK, 55, 2, 0

-1 Start saving dynamic data 
10330, BSAV, 1, .4975, THROTTLE 06, 1, 2, 6, 27

-1 intersection 20 (distance $10350 \mathrm{~m}$ from start), full intersection, left turn only allowed, non signalised

$10010, \mathrm{I}, 2,340,3,2,2$

10010, SIGN, 13, 280, 0, 1, 0, 0

10200, PR, C:ISTISIM ISOUNDIIntersection Left.wav, 0, 10

10336, PR, C:ZAMBDATA\Driver TraininglEssam Input Files\STISIM - Sound 13 SEC.WAV, 0, 2

-1 Conflict traffic on near lane (left lane of opposite direction)

10220, A, $22,220,-1.85, * 18 \sim 35$

$10230, \mathrm{~A}, 21,220,-1.85, * 18 \sim 35$

10245, A, $21,220,-1.85, * 18 \sim 35$

10270, A, 21, 220, $-1.85, * 18 \sim 35$

10310, A, 21, 220, $-1.85, * 18 \sim 35$

$10325, \mathrm{~A}, 21,220,-1.85, * 18 \sim 35$

10335, A, 21, 220, $-1.85, * 18 \sim 35$

-1 Conflict traffic on far lane (right lane of opposite direction)

$10225, \mathrm{~A}, 22,220,-5.55, * 18 \sim 35$

10240, A, $22,220,-5.55, * 18 \sim 35$

10300, A, $22,220,-5.55, * 18 \sim 35$

10330, A, 21, 220, $-5.55, * 18 \sim 35$

-1 The driver must turn before the arrival of these two vehicles

10335, A, 19, 360, $-1.85, * 18 \sim 35$

$10335, \mathrm{~A}, 19,370,-5.55, * 18 \sim 35$

-1 Other vehicles on near lane (left lane of opposite direction)

$10335, \mathrm{~A}, 19,385,-1.85, * 18 \sim 35$

10335, A, 19, 400, $-1.85, * 18 \sim 35$

10335, A, 19, 410, $-1.85, * 18 \sim 35$

$10335, \mathrm{~A}, 19,430,-1.85, * 18 \sim 35$

$10335, \mathrm{~A}, 19,440,-1.85, * 18 \sim 35$

$10335, \mathrm{~A}, 19,455,-1.85, * 18 \sim 35$

$10335, \mathrm{~A}, 18,465,-1.85, * 18 \sim 35$

$10335, \mathrm{~A}, 19,480,-1.85, * 18 \sim 35$

$10335, \mathrm{~A}, 18,520,-1.85, * 18 \sim 35$

$10335, \mathrm{~A}, 19,540,-1.85, * 18 \sim 35$

$10335, \mathrm{~A}, 19,555,-1.85, * 18 \sim 35$

-1 Other vehicles on far lane (right lane of opposite direction) 10335, A, 19, 385, $-5.55, * 18 \sim 35$

$10335, \mathrm{~A}, 19,405,-5.55, * 18 \sim 35$

$10335, \mathrm{~A}, 19,425,-5.55, * 18 \sim 35$

10335, A, 19, 445, $-5.55, * 18 \sim 35$

$10335, \mathrm{~A}, 19,465,-5.55, * 18 \sim 35$ 
10335, A, 19, 485, $-5.55, * 18 \sim 35$

10335, A, 19, 505, $-5.55, * 18 \sim 35$

10335, A, 19, 525, $-5.55, * 18 \sim 35$

-1 End saving dynamic data

10369, ESAV

-1 Control Vehicle for the end of the simulation

10370, CV, 21, 0

-1 Trees

0, TREE, 90, $0, * 1 \sim 18,9.45,18.29,0$

375, TREE, $80,0, * 1 \sim 18,9.45,18.29,0$

805, TREE, 95, $0, * 1 \sim 18,9.45,18.29,0$

1275, TREE, $85,0, * 1 \sim 18,9.45,18.29,0$

1805, TREE, $75,0, * 1 \sim 18,9.45,18.29,0$

2290, TREE, $0,0, * 1 \sim 18,9.45,18.29,0$

3365, TREE, 70, $0, * 1 \sim 18,9.45,18.29,0$

3740, TREE, $0,0, * 1 \sim 18,9.45,18.29,0$

3760, TREE, $70,0, * 1 \sim 18,9.45,18.29,0$

5170, TREE, $0,0, * 1 \sim 18,9.45,18.29,0$

-1 Buildings

360, BLDG, 250, -14.59, $\mathrm{H}^{*} 2 ; 4 \sim 7 ; 9 ; 13$

360, BLDG, 320, -14.59, $\mathrm{H}^{*} 2 ; 4 \sim 7 ; 9 ; 13$

360, BLDG, 220, 14.59, $\mathrm{H}^{*} 2 ; 4 \sim 7 ; 9 ; 13$

360, BLDG, 300, 14.59, H*2;4 7;9;13

3154.36, BLDG, 242.02, 19.99, G*1 13

3154.36, BLDG, 282.02, 19.99, B*1 5;9;13 15

3154.36, BLDG, 322.02, 19.99, B*1 5;9;13 15

3154.36, BLDG, 372.02, 19.99, $\mathrm{B}^{*} 1 \sim 5 ; 9 ; 13 \sim 15$

3154.36, BLDG, 422.02, 19.99, B*1 5;9;13 15

3154.36, BLDG, 462.02, 19.99, G*1 13

3154.36, BLDG, 502.02, 19.99, B*1 5;9;13 15 
3154.36, BLDG, 552.02, 19.99, G*1 13

3154.36, BLDG, 612.02, 19.99, G*1 13

3154.36, BLDG, 662.02, 19.99, B*1 5;9;13 15

3154.36, BLDG, 722.02, 19.99, G*1 13

3154.36, BLDG, 812.02, 19.99, B*1 5;9;13 15

3154.36, BLDG, 892.02, 19.99, G*1 13

3154.36, BLDG, 247.02, -19.99, B*1 5;9;13 15

3154.36, BLDG, 285.02, -19.99, G*1 13

3154.36, BLDG, 326.02, -19.99, G*1 13

3154.36, BLDG, 372.02, -19.99, G*1 13

3154.36, BLDG, 432.02, -19.99, B*1 5;9;13 15

3154.36, BLDG, 522.02, -19.99, B4

3154.36, BLDG, 557.02, -19.99, B*1 5;9;13 15

3154.36, BLDG, 612.02, -19.99, G*1 13

3154.36, BLDG, 662.02, -19.99, G*1 13

3154.36, BLDG, 722.02, -19.99, G*1 13

3154.36, BLDG, 812.02, -19.99, G*1 13

3154.36, BLDG, 892.02, -19.99, G*1 13

3355, BLDG, 129.56, -18.29, H*2;4 7;9;13

3355, BLDG, 159.56, -18.29, H*2;4 7;9;13

3355, BLDG, 199.56, -18.29, $\mathrm{H}^{*} 2 ; 4 \sim 7 ; 9 ; 13$

3355, BLDG, 239.56, -18.29, $\mathrm{H}^{*} 2 ; 4 \sim 7 ; 9 ; 13$

3355, BLDG, 269.56, -18.29, $\mathrm{H}^{*} 2 ; 4 \sim 7 ; 9 ; 13$

3355, BLDG, 339.56, -18.29, $\mathrm{H}^{* 2 ; 4 ~ 7 ; 9 ; 13}$

3355, BLDG, 129.56, 18.29, $\mathrm{H}^{*} 2 ; 4 \sim 7 ; 9 ; 13$

3355, BLDG, 169.56, 18.29, $\mathrm{H}^{*} 2 ; 4 \sim 7 ; 9 ; 13$

3355, BLDG, 199.56, 18.29, $\mathrm{H}^{*} 2 ; 4 \sim 7 ; 9 ; 13$

3355, BLDG, 249.56, 18.29, $\mathrm{H}^{*} 2 ; 4 \sim 7 ; 9 ; 13$

3355, BLDG, 279.56, 18.29, H*2;4 7;9;13

3355, BLDG, 339.56, 18.29, H*2;4 7;9;13

3755, BLDG, 129.56, -18.29, $\mathrm{H}^{*} 2 ; 4 \sim 7 ; 9 ; 13$

3755, BLDG, 159.56, -18.29, $\mathrm{H}^{*} 2 ; 4 \sim 7 ; 9 ; 13$

3755, BLDG, 199.56, -18.29, $\mathrm{H}^{*} 2 ; 4 \sim 7 ; 9 ; 13$

3755, BLDG, 239.56, -18.29, $\mathrm{H}^{*} 2 ; 4 \sim 7 ; 9 ; 13$

3755, BLDG, 269.56, -18.29, $\mathrm{H}^{*} 2 ; 4 \sim 7 ; 9 ; 13$

3755, BLDG, 339.56, -18.29, $\mathrm{H}^{*} 2 ; 4 \sim 7 ; 9 ; 13$

3755, BLDG, 379.56, -18.29, $\mathrm{H}^{* 2 ; 4 \sim 7 ; 9 ; 13}$

3755, BLDG, 439.56, -18.29, H*2;4 7;9;13 
3755, BLDG, 129.56, 18.29, H*2;4 7;9;13 3755, BLDG, 169.56, 18.29, $\mathrm{H}^{*} 2 ; 4 \sim 7 ; 9 ; 13$ 3755, BLDG, 199.56, 18.29, $\mathrm{H}^{*} 2 ; 4 \sim 7 ; 9 ; 13$ 3755, BLDG, 249.56, 18.29, $\mathrm{H}^{*} 2 ; 4 \sim 7 ; 9 ; 13$ 3755, BLDG, 279.56, 18.29, $\mathrm{H}^{*} 2 ; 4 \sim 7 ; 9 ; 13$ 3755, BLDG, 339.56, 18.29, $\mathrm{H}^{*} 2 ; 4 \sim 7 ; 9 ; 13$ 3755, BLDG, 389.56, 18.29, $\mathrm{H}^{*} 2 ; 4 \sim 7 ; 9 ; 13$ 3755, BLDG, 439.56, 18.29, $\mathrm{H}^{*} 2 ; 4 \sim 7 ; 9 ; 13$

4255, BLDG, 129.56, -18.29, $\mathrm{H}^{*} 2 ; 4 \sim 7 ; 9 ; 13$ 4255, BLDG, 159.56, -18.29, H*2;4 7;9;13 4255, BLDG, 199.56, -18.29, $\mathrm{H}^{* 2 ; 4 \sim 7 ; 9 ; 13}$ 4255, BLDG, 239.56, -18.29, H*2;4 7;9;13 4255, BLDG, 269.56, -18.29, H*2;4 7;9;13 4255, BLDG, 339.56, -18.29, $\mathrm{H}^{*} 2 ; 4 \sim 7 ; 9 ; 13$ 4255, BLDG, 389.56, -18.29, $\mathrm{H}^{* 2 ; 4 \sim 7 ; 9 ; 13}$ 4255, BLDG, 429.56, -18.29, $\mathrm{H}^{* 2 ; 4 \sim 7 ; 9 ; 13}$

4255, BLDG, 129.56, 18.29, H*2;4 7;9;13 4255, BLDG, 169.56, 18.29, $\mathrm{H}^{*} 2 ; 4 \sim 7 ; 9 ; 13$ 4255, BLDG, 199.56, 18.29, $\mathrm{H}^{*} 2 ; 4 \sim 7 ; 9 ; 13$ 4255, BLDG, 249.56, 18.29, H*2;4 7;9;13 4255, BLDG, 279.56, 18.29, $\mathrm{H}^{*} 2 ; 4 \sim 7 ; 9 ; 13$ 4255, BLDG, 339.56, 18.29, $\mathrm{H}^{*} 2 ; 4 \sim 7 ; 9 ; 13$ 4255, BLDG, 379.56, 18.29, $\mathrm{H}^{*} 2 ; 4 \sim 7 ; 9 ; 13$ 4255, BLDG, 419.56, 18.29, H*2;4 7;9;13

4755, BLDG, 129.56, -18.29, $\mathrm{H}^{*} 2 ; 4 \sim 7 ; 9 ; 13$ 4755, BLDG, 159.56, -18.29, $\mathrm{H}^{*} 2 ; 4 \sim 7 ; 9 ; 13$ 4755, BLDG, 199.56, -18.29, $\mathrm{H}^{* 2 ; 4 ~ 7 ; 9 ; 13}$ 4755, BLDG, 239.56, -18.29, $\mathrm{H}^{* 2 ; 4 ~ 7 ; 9 ; 13}$ 4755, BLDG, 269.56, -18.29, $\mathrm{H}^{*} 2 ; 4 \sim 7 ; 9 ; 13$ 4755, BLDG, 339.56, -18.29, $\mathrm{H}^{*} 2 ; 4 \sim 7 ; 9 ; 13$ 4755, BLDG, 389.56, -18.29, H*2;4 7;9;13 4755, BLDG, 429.56, -18.29, H*2;4 7;9;13 4755, BLDG, 129.56, 18.29, H*2;4 7;9;13 4755, BLDG, 169.56, 18.29, $\mathrm{H}^{*} 2 ; 4 \sim 7 ; 9 ; 13$ 4755, BLDG, 199.56, 18.29, $\mathrm{H}^{*} 2 ; 4 \sim 7 ; 9 ; 13$ 4755, BLDG, 249.56, 18.29, $\mathrm{H}^{*} 2 ; 4 \sim 7 ; 9 ; 13$ 4755, BLDG, 279.56, 18.29, $\mathrm{H}^{*} 2 ; 4 \sim 7 ; 9 ; 13$ 4755, BLDG, 339.56, 18.29, $\mathrm{H}^{*} 2 ; 4 \sim 7 ; 9 ; 13$ 4755, BLDG, 379.56, 18.29, $\mathrm{H}^{*} 2 ; 4 \sim 7 ; 9 ; 13$ 4755, BLDG, 419.56, 18.29, H*2;4 7;9;13 
4755, BLDG, 479.56, 18.29, $\mathrm{H}^{*} 2 ; 4 \sim 7 ; 9 ; 13$

4755, BLDG, 499.56, 18.29, $\mathrm{H}^{*} 2 ; 4 \sim 7 ; 9 ; 13$

5305, BLDG, 52.02, 22.99, $\mathrm{B}^{*} 1 \sim 5 ; 9 ; 13 \sim 15$ 5305, BLDG, 82.02, 22.99, $\mathrm{B}^{*} 1 \sim 5 ; 9 ; 13 \sim 15$ 5305, BLDG, 112.02, 22.99, B*1 5;9;13 15 5305, BLDG, 152.02, 22.99, $\mathrm{B}^{*} 1 \sim 5 ; 9 ; 13 \sim 15$ 5305, BLDG, 192.02, 22.99, $\mathrm{B}^{*} 1 \sim 5 ; 9 ; 13 \sim 15$ 5305, BLDG, 252.02, 22.99, $\mathrm{B} * 1 \sim 5 ; 9 ; 13 \sim 15$ 5305, BLDG, 295.02, 22.99, B*1 5;9;13 15 5305, BLDG, 322.02, 22.99, B*1 5;9;13 15 5305, BLDG, 362.02, 22.99, B*1 5;9;13 15 5305, BLDG, 397.02, 22.99, B*1 5;9;13 15 5305, BLDG, 422.02, 22.99, B*1 5;9;13 15

5305, BLDG, 32.02, -22.99, B9

5305, BLDG, 82.02, -22.99, B*1 5;13 15

5305, BLDG, 162.02, -22.99, B*1 5;9;13 15

5305, BLDG, 202.02, -221.99, B*1 5;9;13 15 5305, BLDG, 242.02, $-22.99, \mathrm{~B} * 1 \sim 5 ; 9 ; 13 \sim 15$ 5305, BLDG, 282.02, -22.99, $\mathrm{B} * 1 \sim 5 ; 9 ; 13 \sim 15$ 5305, BLDG, 312.02, -22.99, $\mathrm{B} * 1 \sim 5 ; 9 ; 13 \sim 15$ 5305, BLDG, 332.02, -22.99, B*1 5;9;13 15 5305, BLDG, 372.02, -22.99, B*1 5;9;13 15 5305, BLDG, 402.02, $-22.99, \mathrm{~B}^{*} 1 \sim 5 ; 9 ; 13 \sim 15$ 5305, BLDG, 422.02, -22.99, $\mathrm{B} * 1 \sim 5 ; 9 ; 13 \sim 15$

5815, BLDG, 72.02, 22.99, G*1 13

5815, BLDG, 122.02, 22.99, $\mathrm{G}^{*} 1 \sim 13$

5815, BLDG, 162.02, 22.99, B*1 5;9;13 15

5815, BLDG, 202.02, 22.99, B*1 5;9;13 15

5815, BLDG, 242.02, 22.99, G*1 13

5815, BLDG, 282.02, 22.99, B*1 5;9;13 15

5815, BLDG, 322.02, 22.99, B*1 5;9;13 15

5815, BLDG, 372.02, 22.99, B*1 5;9;13 15

5815, BLDG, 422.02, 22.99, B*1 5;9;13 15

5815, BLDG, 462.02, 22.99, G*1 13

5815, BLDG, 502.02, 22.99, B*1 5;9;13 15

5815, BLDG, 552.02, 22.99, G*1 13

5815, BLDG, 612.02, 22.99, $\mathrm{G}^{*} 1 \sim 13$

5815, BLDG, 662.02, 22.99, B*1 5;9;13 15

5815, BLDG, 722.02, 22.99, G*1 13

5815, BLDG, 812.02, 22.99, $\mathrm{B} * 1 \sim 5 ; 9 ; 13 \sim 15$

5815, BLDG, 892.02, 22.99, G*1 13 
5815, BLDG, 82.02, -22.99, B*1 5;9;13 15

5815, BLDG, 122.02, $-22.99, \mathrm{G}^{*} 1 \sim 13$

5815, BLDG, 172.02, -22.99, B*1 5;9;13 15

5815, BLDG, 206.02, -22.99, G*1 13

5815, BLDG, 247.02, -22.99, $\mathrm{B}^{*} 1 \sim 5 ; 9 ; 13 \sim 15$

5815, BLDG, 285.02, -22.99, G*1 13

5815, BLDG, 326.02, $-22.99, \mathrm{G}^{*} 1 \sim 13$

5815, BLDG, 372.02, $-22.99, \mathrm{G}^{*} 1 \sim 13$

5815, BLDG, 432.02, -22.99, B*1 5;9;13 15

5815, BLDG, 522.02, -22.99, B4

5815, BLDG, 557.02, -22.99, B*1 5;9;13 15

5815, BLDG, 612.02, $-22.99, \mathrm{G}^{*} 1 \sim 13$

5815, BLDG, 662.02, $-22.99, \mathrm{G}^{*} 1 \sim 13$

5815, BLDG, 722.02, $-22.99, \mathrm{G}^{*} 1 \sim 13$

5815, BLDG, 812.02, $-22.99, \mathrm{G}^{*} 1 \sim 13$

5815, BLDG, 892.02, $-22.99, \mathrm{G}^{*} 1 \sim 13$

6315, BLDG, 72.02, 22.99, G*1 13

6315, BLDG, 122.02, 22.99, G*1 13

6315, BLDG, 162.02, 22.99, $\mathrm{B} * 1 \sim 5 ; 9 ; 13 \sim 15$

6315, BLDG, 202.02, 22.99, $\mathrm{B} * 1 \sim 5 ; 9 ; 13 \sim 15$

6315, BLDG, 242.02, 22.99, G*1 13

6315, BLDG, 282.02, 22.99, $\mathrm{B} * 1 \sim 5 ; 9 ; 13 \sim 15$

6315, BLDG, 322.02, 22.99, B*1 5;9;13 15

6315, BLDG, 372.02, 22.99, B*1 5;9;13 15

6315, BLDG, 422.02, 22.99, B*1 5;9;13 15

6315, BLDG, 462.02, 22.99, $\mathrm{G}^{*} 1 \sim 13$

6315, BLDG, 502.02, 22.99, B*1 5;9;13 15

6315, BLDG, 552.02, 22.99, G*1 13

6315, BLDG, 612.02, 22.99, $\mathrm{G}^{*} 1 \sim 13$

6315, BLDG, 662.02, 22.99, B*1 5;9;13 15

6315, BLDG, 722.02, 22.99, G*1 13

6315, BLDG, 812.02, 22.99, B*1 5;9;13 15

6315, BLDG, 892.02, 22.99, G*1 13

6315, BLDG, 82.02, -22.99, B*1 5;9;13 15

6315, BLDG, 122.02, -22.99, G*1 13

6315, BLDG, 172.02, -22.99, $\mathrm{B} * 1 \sim 5 ; 9 ; 13 \sim 15$

6315, BLDG, 206.02, -22.99, G*1 13

6315, BLDG, 247.02, -22.99, $\mathrm{B}^{*} 1 \sim 5 ; 9 ; 13 \sim 15$

6315, BLDG, 285.02, -22.99, G*1 13

6315, BLDG, 326.02, -22.99, G*1 13

6315, BLDG, 372.02, -22.99, G*1 13

6315, BLDG, 432.02, -22.99, B*1 5;9;13 15

6315, BLDG, 522.02, -22.99, B4

6315, BLDG, 557.02, -22.99, B*1 5;9;13 15

6315, BLDG, 612.02, -22.99, G*1 13

6315, BLDG, 662.02, $-22.99, \mathrm{G}^{*} 1 \sim 13$

6315, BLDG, 722.02, $-22.99, \mathrm{G}^{*} 1 \sim 13$

6315, BLDG, 812.02, -22.99, G*1 13 
6315, BLDG, 892.02, -22.99, G*1 13

6815, BLDG, 72.02, 22.99, G*1 13

6815, BLDG, 122.02, 22.99, G*1 13

6815, BLDG, 162.02, 22.99, B*1 5;9;13 15

6815, BLDG, 202.02, 22.99, B*1 5;9;13 15

6815, BLDG, 242.02, 22.99, G*1 13

6815, BLDG, 282.02, 22.99, B*1 5;9;13 15

6815, BLDG, 322.02, 22.99, $\mathrm{B}^{*} 1 \sim 5 ; 9 ; 13 \sim 15$

6815, BLDG, 372.02, 22.99, $\mathrm{B}^{*} 1 \sim 5 ; 9 ; 13 \sim 15$

6815, BLDG, 422.02, 22.99, $\mathrm{B} * 1 \sim 5 ; 9 ; 13 \sim 15$

6815, BLDG, 462.02, 22.99, G*1 13

6815, BLDG, 502.02, 22.99, B*1 5;9;13 15

6815, BLDG, 552.02, 22.99, G*1 13

6815, BLDG, 612.02, 22.99, G*1 13

6815, BLDG, 662.02, 22.99, B*1 5;9;13 15

6815, BLDG, 722.02, 22.99, G*1 13

6815, BLDG, 812.02, 22.99, B*1 5;9;13 15

6815, BLDG, 892.02, 22.99, G*1 13

6815, BLDG, 82.02, -22.99, B*1 5;9;13 15

6815, BLDG, 122.02, $-22.99, \mathrm{G}^{*} 1 \sim 13$

6815, BLDG, 172.02, -22.99, $\mathrm{B} * 1 \sim 5 ; 9 ; 13 \sim 15$

6815, BLDG, 206.02, $-22.99, \mathrm{G}^{*} 1 \sim 13$

6815, BLDG, 247.02, -22.99, $\mathrm{B} * 1 \sim 5 ; 9 ; 13 \sim 15$

6815, BLDG, 285.02, $-22.99, \mathrm{G}^{*} 1 \sim 13$

6815, BLDG, 326.02, $-22.99, \mathrm{G}^{*} 1 \sim 13$

6815, BLDG, 372.02, $-22.99, \mathrm{G}^{*} 1 \sim 13$

6815, BLDG, 432.02, -22.99, B*1 5;9;13 15

6815, BLDG, 522.02, -22.99, B4

6815, BLDG, 557.02, -22.99, B*1 5;9;13 15

6815, BLDG, 592.02, $-22.99, \mathrm{G}^{*} 1 \sim 13$

6815, BLDG, 712.02, $-22.99, \mathrm{G}^{*} 1 \sim 13$

6815, BLDG, 892.02, -22.99, G*1 13

7504.36, BLDG, 72.02, 22.99, G*1 13

7504.36, BLDG, 122.02, 22.99, $\mathrm{G}^{*} 1 \sim 13$

7504.36, BLDG, 162.02, 22.99, $\mathrm{B} * 1 \sim 5 ; 9 ; 13 \sim 15$

7504.36, BLDG, 202.02, 22.99, B*1 5;9;13 15

7504.36, BLDG, 242.02, 22.99, G*1 13

7504.36, BLDG, 282.02, 22.99, B*1 5;9;13 15

7504.36, BLDG, 322.02, 22.99, B*1 5;9;13 15

7504.36, BLDG, 372.02, 22.99, B*1 5;9;13 15

7504.36, BLDG, 422.02, 22.99, B*1 5;9;13 15

7504.36, BLDG, 462.02, 22.99, G*1 13

7504.36, BLDG, 502.02, 22.99, B*1 5;9;13 15

7504.36, BLDG, 552.02, 22.99, G*1 13

7504.36, BLDG, 612.02, 22.99, G*1 13

7504.36, BLDG, 662.02, 22.99, B*1 5;9;13 15

7504.36, BLDG, 722.02, 22.99, G*1 13 
7504.36, BLDG, 812.02, 22.99, B*1 5;9;13 15

7504.36, BLDG, 892.02, 22.99, G*1 13

7504.36, BLDG, 82.02, -22.99, B*1 5;9;13 15

7504.36, BLDG, 122.02, -22.99, $\mathrm{G}^{*} 1 \sim 13$

7504.36, BLDG, 172.02, -22.99, B*1 5;9;13 15

7504.36, BLDG, 206.02, -22.99, G*1 13

7504.36, BLDG, 247.02, -22.99, B*1 5;9;13 15

7504.36, BLDG, 285.02, -22.99, G*1 13

7504.36, BLDG, 326.02, -22.99, G*1 13

7504.36, BLDG, 372.02, -22.99, G*1 13

7504.36, BLDG, 432.02, -22.99, $\mathrm{B} * 1 \sim 5 ; 9 ; 13 \sim 15$

7504.36, BLDG, 522.02, -22.99, B4

7504.36, BLDG, 557.02, -22.99, B*1 5;9;13 15

7504.36, BLDG, 612.02, -22.99, G*1 13

7504.36, BLDG, 662.02, -22.99, G*1 13

7504.36, BLDG, 722.02, -22.99, G*1 13

7504.36, BLDG, 812.02, -22.99, G*1 13

7504.36, BLDG, 892.02, -22.99, G*1 13

8015, BLDG, 202.02, 22.99, B*1 5;9;13 15

8015, BLDG, 242.02, 22.99, G*1 13

8015, BLDG, 282.02, 22.99, B*1 5;9;13 15

8015, BLDG, 322.02, 22.99, B*1 5;9;13 15

8015, BLDG, 372.02, 22.99, B*1 5;9;13 15

8015, BLDG, 412.02, 22.99, B*1 5;9;13 15

8015, BLDG, 442.02, 22.99, $\mathrm{G}^{*} 1 \sim 13$

8015, BLDG, 522.02, 22.99, B*1 5;9;13 15

8015, BLDG, 572.02, 22.99, G*1 13

8015, BLDG, 612.02, 22.99, $\mathrm{G}^{*} 1 \sim 13$

8015, BLDG, 662.02, 22.99, $\mathrm{B} * 1 \sim 5 ; 9 ; 13 \sim 15$

8015, BLDG, 722.02, 22.99, G*1 13

8015, BLDG, 812.02, 22.99, B*1 5;9;13 15

8015, BLDG, 206.02, -22.99, G*1 13

8015, BLDG, 247.02, -22.99, $\mathrm{B} * 1 \sim 5 ; 9 ; 13 \sim 15$

8015, BLDG, 285.02, $-22.99, \mathrm{G}^{*} 1 \sim 13$

8015, BLDG, 326.02, $-22.99, \mathrm{G}^{*} 1 \sim 13$

8015, BLDG, 372.02, -22.99, G*1 13

8015, BLDG, 412.02, -22.99, B*1 5;9;13 15

8015, BLDG, 542.02, -22.99, B4

8015, BLDG, 587.02, -22.99, B*1 5;9;13 15

8015, BLDG, 642.02, -22.99, G*1 13

8015, BLDG, 682.02, $-22.99, \mathrm{G}^{*} 1 \sim 13$

8015, BLDG, 762.02, $-22.99, \mathrm{G}^{*} 1 \sim 13$

8015, BLDG, 812.02, $-22.99, \mathrm{G}^{*} 1 \sim 13$

8015, BLDG, 892.02, -22.99, G*1 13

8515, BLDG, 202.02, 19.99, B*1 5;9;13 15

8515, BLDG, 242.02, 19.99, G*1 13 
8515, BLDG, 282.02, 19.99, B*1 5;9;13 15

8515, BLDG, 322.02, 19.99, B*1 5;9;13 15

8515, BLDG, 372.02, 19.99, B*1 5;9;13 15

8515, BLDG, 572.02, 19.99, G*1 13

8515, BLDG, 622.02, 19.99, G*1 13

8515, BLDG, 662.02, 19.99, B*1 5;9;13 15

8515, BLDG, 722.02, 19.99, G*1 13

- 8515, BLDG, 812.02, 19.99, B*1 5;9;13 15

8515, BLDG, 892.02, 19.99, G*1 13

8515, BLDG, 206.02, -19.99, G*1 13

8515, BLDG, 247.02, -19.99, B*1 5;9;13 15

8515, BLDG, 285.02, -19.99, G*1 13

8515, BLDG, 326.02, -19.99, G*1 13

8515, BLDG, 372.02, -19.99, G*1 13

8515, BLDG, 432.02, -19.99, B*1 5;9;13 15

- 8515, BLDG, 522.02, -19.99, B4

8515, BLDG, 567.02, -19.99, B*1 5;9;13 15

8515, BLDG, 622.02, -19.99, G*1 13

8515, BLDG, 662.02, -19.99, G*1 13

8515, BLDG, 722.02, $-19.99, \mathrm{G}^{*} 1 \sim 13$

- 8515, BLDG, 812.02, -19.99, G*1 13

8515, BLDG, 892.02, -19.99, G*1 13

9015, BLDG, 202.02, 19.99, B*1 5;9;13 15

9015, BLDG, 242.02, 19.99, G*1 13

9015, BLDG, 322.02, 19.99, B*1 5;9;13 15

9015, BLDG, 372.02, 19.99, B*1 5;9;13 15

9015, BLDG, 422.02, 19.99, B*1 5;9;13 15

9015, BLDG, 462.02, 19.99, G*1 13

9015, BLDG, 502.02, 19.99, B*1 5;9;13 15

9015, BLDG, 552.02, 19.99, G*1 13

9015, BLDG, 612.02, 19.99, G*1 13

9015, BLDG, 662.02, 19.99, B*1 5;9;13 15

9015, BLDG, 722.02, 19.99, G*1 13

9015, BLDG, 812.02, 19.99, B*1 5;9;13 15

9015, BLDG, 892.02, 19.99, G*1 13

9015, BLDG, 206.02, -19.99, G*1 13

9015, BLDG, 247.02, -19.99, $\mathrm{B} * 1 \sim 5 ; 9 ; 13 \sim 15$

9015, BLDG, 336.02, -19.99, G*1 13

9015, BLDG, 372.02, -19.99, G*1 13

9015, BLDG, 432.02, -19.99, B*1 5;9;13 15

9015, BLDG, 522.02, -19.99, B4

9015, BLDG, 557.02, -19.99, B*1 5;9;13 15

9015, BLDG, 612.02, $-19.99, \mathrm{G}^{*} 1 \sim 13$

9015, BLDG, 662.02, -19.99, G*1 13

9015, BLDG, 722.02, -19.99, G*1 13

9015, BLDG, 812.02, -19.99, G*1 13

9015, BLDG, 892.02, -19.99, G*1 13 
9315, BLDG, 202.02, 19.99, B*1 5;9;13 15 9315, BLDG, 242.02, 19.99, G*1 13

9315, BLDG, 282.02, 19.99, B*1 5;9;13 15

9315, BLDG, 322.02, 19.99, B*1 5;9;13 15

9315, BLDG, 372.02, 19.99, $\mathrm{B} * 1 \sim 5 ; 9 ; 13 \sim 15$

9315, BLDG, 502.02, 19.99, B*1 5;9;13 15

9315, BLDG, 552.02, 19.99, G*1 13

9315, BLDG, 612.02, 19.99, G*1 13

9315, BLDG, 662.02, 19.99, $\mathrm{B} * 1 \sim 5 ; 9 ; 13 \sim 15$

9315, BLDG, 722.02, 19.99, G*1 13

9315, BLDG, 812.02, 19.99, B*1 5;9;13 15

9315, BLDG, 892.02, 19.99, G*1 13

9315, BLDG, 206.02, -19.99, G*1 13

9315, BLDG, 247.02, -19.99, $\mathrm{B}^{*} 1 \sim 5 ; 9 ; 13 \sim 15$

9315, BLDG, 285.02, -19.99, G*1 13

9315, BLDG, 326.02, -19.99, G*1 13

9315, BLDG, 372.02, -19.99, G*1 13

9315, BLDG, 522.02, -19.99, B4

9315, BLDG, 557.02, -19.99, B*1 5;9;13 15

9315, BLDG, 612.02, -19.99, G*1 13

9315, BLDG, 662.02, -19.99, G*1 13

9315, BLDG, 722.02, -19.99, G*1 13

9315, BLDG, 812.02, -19.99, G*1 13

9315, BLDG, 892.02, -19.99, G*1 13

9765, BLDG, 202.02, 19.99, B*1 5;9;13 15

9765, BLDG, 242.02, 19.99, G*1 13

9765, BLDG, 282.02, 19.99, B*1 5;9;13 15

9765, BLDG, 322.02, 19.99, B*1 5;9;13 15

9765, BLDG, 372.02, 19.99, B*1 5;9;13 15

9765, BLDG, 422.02, 19.99, B*1 5;9;13 15

-9765, BLDG, 462.02, 19.99, G*1 13

9765, BLDG, 502.02, 19.99, B*1 5;9;13 15

9765, BLDG, 552.02, 19.99, G*1 13

9765, BLDG, 662.02, 19.99, B*1 5;9;13 15

9765, BLDG, 722.02, 19.99, G*1 13

9765, BLDG, 812.02, 19.99, B*1 5;9;13 15

9765, BLDG, 892.02, 19.99, G*1 13

9765, BLDG, 206.02, -19.99, G*1 13

9765, BLDG, 247.02, -19.99, $\mathrm{B}^{*} 1 \sim 5 ; 9 ; 13 \sim 15$

9765, BLDG, 285.02, -19.99, G*1 13

9765, BLDG, 326.02, -19.99, G*1 13

9765, BLDG, 372.02, -19.99, G*1 13

9765, BLDG, 422.02, -19.99, B*1 5;9;13 15

9765, BLDG, 722.02, $-19.99, \mathrm{G}^{*} 1 \sim 13$

9765, BLDG, 812.02, $-19.99, \mathrm{G}^{*} 1 \sim 13$

9765, BLDG, 892.02, -19.99, G*1 13 
10354.36, BLDG, 202.02, 19.99, B*1 5;9;13 15

10354.36, BLDG, 242.02, 19.99, G*1 13

10354.36, BLDG, 282.02, 19.99, B*1 5;9;13 15

10354.36, BLDG, 322.02, 19.99, B*1 5;9;13 15

10354.36, BLDG, 372.02, 19.99, B*1 5;9;13 15

10354.36, BLDG, 422.02, 19.99, B*1 5;9;13 15

-10354.36, BLDG, 462.02, 19.99, G*1 13

10354.36, BLDG, 502.02, 19.99, B*1 5;9;13 15

10354.36, BLDG, 552.02, 19.99, G*1 13

10354.36, BLDG, 612.02, 19.99, G*1 13

10354.36, BLDG, 662.02, 19.99, B*1 5;9;13 15

10354.36, BLDG, 722.02, 19.99, G*1 13

10354.36, BLDG, 812.02, 19.99, B*1 5;9;13 15

10354.36, BLDG, 892.02, 19.99, G*1 13

10354.36, BLDG, 206.02, -19.99, G*1 13

10354.36, BLDG, 247.02, -19.99, $\mathrm{B} * 1 \sim 5 ; 9 ; 13 \sim 15$

10354.36, BLDG, 285.02, -19.99, G*1 13

10354.36, BLDG, 326.02, -19.99, G*1 13

10354.36, BLDG, 372.02, -19.99, G*1 13

-10354.36, BLDG, 432.02, -19.99, B*1 5;9;13 15

10354.36, BLDG, 522.02, -19.99, B4

10354.36, BLDG, 557.02, -19.99, B*1 5;9;13 15

10354.36, BLDG, 612.02, -19.99, G*1 13

10354.36, BLDG, 662.02, -19.99, G*1 13

10354.36, BLDG, 722.02, -19.99, G*1 13

10354.36, BLDG, 812.02, -19.99, G*1 13

10354.36, BLDG, 892.02, -19.99, G*1 13

10500, ES 


\section{Appendix "B"}

Simulation Scenario for TWSC Situations 


\section{METRIC}

-1 Two-lane Rural Road

0 , ROAD , 3.7, 2, 1, 1, 0.15, 0.85, .7, .12, .16, 0, -2, -2, 0, 0, 0, 0, -8, 1.52, -8, 1.52, 0, 0, 0 , C:ISTISIM\DatalTextures\Grass08.Jpg, 25, C:ISTISIM\DatalTextures\Dirt03.Jpg, 12, C:ISTISIM\DatalTextures\Grass08.Jpg, 25

-1 Vehicles travelling on the same direction of the driver

$15, \mathrm{~V}, 18,260,1.85,1,21$

$80, \mathrm{~V}, 20,260,1.85,1,26$

$140, \mathrm{~V}, 19,260,1.85,1, * 18 \sim 35$

$180, \mathrm{~V}, 19,260,1.85,1, * 18 \sim 35$

-1 Control Vehicle for Intersection 01

$0, \mathrm{CV}, 18,0$

282, CV, 0, 0

283, BRK, 45, 1, 40

323, BRK, 70, 2, 0

-1 Start saving dynamic data

330, BSAV, 1, .4975, THROTTLE 01, 1, 6, 27

-1 intersection 01 (distance $350 \mathrm{~m}$ from start), full intersection, going straight, Two-way Stop controlled

10, I, 0, 340, 1, 0, 0

10, SIGN, 2, 200, 0, 1, 0, 0

10, SIGN, 7, 334, 2, 0, 0, 0

200, PR, C:ISTISIMISOUNDUIntersection straight.wav, 0, 10

339, PR, C:IZAMBDATA\Driver TraininglEssam Input FilesISTISIM - Sound $\backslash 11$ SEC.WAV, 0, 2

-1 Cross traffic from left

148.15, CT, 200, 5, -35, 18, L, *18 35, 0

$148.15, \mathrm{CT}, 200,5,-55,18, \mathrm{~L}, * 18 \sim 35,0$

248.15, CT, 100, 5, -35, 18, L, *18 35, 0

$248.15, \mathrm{CT}, 100,5,-55,18, \mathrm{~L}, * 18 \sim 35,0$

$335, \mathrm{CT}, 13.15,5,-40,18, \mathrm{~L}, * 18 \sim 35,0$

$335, \mathrm{CT}, 13.15,5,-85,18, \mathrm{~L}, * 18 \sim 35,0$

$335, \mathrm{CT}, 13.15,5,-115,18, \mathrm{~L}, * 18 \sim 35,0$

$335, \mathrm{CT}, 13.15,5,-145,18, \mathrm{~L}, * 18 \sim 35,0$

$335, \mathrm{CT}, 13.15,5,-172,18, \mathrm{~L}, * 18 \sim 35,0$

335, CT, 13.15, 5, -191, 18, L, *18 35, 0 
335, CT, 13.15, 5, -205, 18, L, *18 35, 0

-1 Cross traffic from right

51.85, CT, 300, 5, 35, 18, R, *18 35, 0

$51.85, \mathrm{CT}, 300,5,75,18, \mathrm{R}, * 18 \sim 35,0$

$51.85, \mathrm{CT}, 300,5,95,18, \mathrm{R}, * 18 \sim 35,0$

51.85, CT, 300, 5, 125, 18, R, *18 35, 0

$335, \mathrm{CT}, 16.85,5,170,18, \mathrm{R}, * 18 \sim 35,0$

-1 The driver must turn before the arrival of these vehicles

335, CT, 13.15, 5, -335, 17, L, 3, 1

$335, \mathrm{CT}, 16.85,5,345,17, \mathrm{R}, 3,1$

-1 Other vehicles from left

335, CT, 13.15, 5, -385, 17, L, *18 35, 1

335, CT, 13.15, 5, $-415,17, \mathrm{~L}, * 18 \sim 35,1$

335, CT, 13.15, 5, -435, 17, L, *18 35, 1

$335, \mathrm{CT}, 13.15,5,-475,17, \mathrm{~L}, * 18 \sim 35,1$

-1 Other vehicles from right

335, CT, $16.85,5,365,17, \mathrm{R}, * 18 \sim 35,1$

$335, \mathrm{CT}, 16.85,5,395,17, \mathrm{R}, * 18 \sim 35,1$

$335, \mathrm{CT}, 16.85,5,425,17, \mathrm{R}, * 18 \sim 35,1$

335, CT, 16.85, 5, 455, 17, R, *18 35, 1

$335, \mathrm{CT}, 16.85,5,475,17, \mathrm{R}, * 18 \sim 35,1$

-1 End saving dynamic data

369, ESAV

-1 Control Vehicle for Intersection 02

$370, \mathrm{CV}, 18,0$

682, CV, 0,0

683, BRK, 45, 1, 40

723, BRK, 70, 2, 0

-1 Start saving dynamic data

730, BSAV, 1, .4975, THROTTLE 02, 1, 6, 27

-1 intersection 02 (distance $750 \mathrm{~m}$ from start), full intersection, right turn, Two-way Stop controlled 
410, I, 0, 340, 1, 0, 0

410, SIGN, 2, 200, 0, 1, 0, 0

410, SIGN, 7, 334, 2, 0, 0, 0

600, PR, C:ISTISIM ISOUNDUIntersection right.wav, 0, 10

739, PR, C:IZAMBDATA|Driver TraininglEssam Input Files\STISIM - Sound 09 SEC.WAV, 0, 2

-1 Cross traffic from left

548.15, CT, 200, 5, -35, 18, L, *18 35, 0

$548.15, \mathrm{CT}, 200,5,-55,18, \mathrm{~L}, * 18 \sim 35,0$

648.15, CT, 100, 5, -35, 18, L, *18 35, 0

648.15, CT, 100, 5, -55, 18, L, *18 35, 0

735, CT, 13.15, 5, -40, 18, L, *18 35, 0

735, CT, $13.15,5,-85,18, \mathrm{~L}, * 18 \sim 35,0$

735, CT, 13.15, 5, -115, 18, L, *18 35, 0

735, CT, 13.15, 5, -135, 18, L, *18 35, 0

735, CT, 13.15, 5, $-152,18, \mathrm{~L}, * 18 \sim 35,0$

735, CT, 13.15, 5, -171, 18, L, *18 35, 0

735, CT, 13.15, 5, -190, 19, L, *18 35, 0

-1 Cross traffic from right

451.85, CT, 300, 5, 35, 18, R, *18 35, 0

451.85, CT, 300, 5, 75, 18, R, *18 35, 0

451.85, CT, 300, 5, 95, 18, R, *18 35, 0

451.85, CT, 300, 5, 125, 18, R, *18 35, 0

735, CT, $16.85,5,170,19, \mathrm{R}, * 18 \sim 35,0$

-1 The driver must turn before the arrival of this vehicle

735, CT, 13.15, 5, -275, 16, L, 3, 1

-1 Other vehicles from left

735, CT, 13.15, 5, -285, 16, L, *18 35, 1

735, CT, 13.15, 5, -315, 16, L, *18 35, 1

735, CT, $13.15,5,-345,16, \mathrm{~L}, * 18 \sim 35,1$

735, CT, 13.15, 5, -395, 16, L, *18 35, 1

735, CT, 13.15, 5, -415, 16, L, *18 35, 1

735, CT, 13.15, 5, -445, 16, L, *18 35, 1

-1 Other vehicles from right

735, CT, 16.85, 5, 365, 17, R, *18 35, 1

735, CT, $16.85,5,395,17, \mathrm{R}, * 18 \sim 35,1$

735, CT, $16.85,5,425,17, \mathrm{R}, * 18 \sim 35,1$

735, CT, $16.85,5,455,17, \mathrm{R}, * 18 \sim 35,1$ 
-1 End saving dynamic data

769, ESAV

-1 Control Vehicle for Intersection 03

$770, \mathrm{CV}, 19,0$

$1182, \mathrm{CV}, 0,0$

$1183, \mathrm{BRK}, 45,1,40$

1223, BRK, 72, 2, 0

-1 Start saving dynamic data

1230, BSAV, $1, .4975$, THROTTLE 03, 1, 6, 27

-1 intersection 03 (distance $1250 \mathrm{~m}$ from start), full intersection, left turn, Two-way Stop controlled

910, I, 0, 340, 1, 0, 0

910, SIGN, 2, 200, 0, 1, 0, 0

910, SIGN, 7, 334, 2, 0, 0, 0

1100, PR, C:ISTISIMISOUND Intersection left.wav, 0, 10

1239, PR, C:IZAMBDATAIDriver TraininglEssam Input Files\STISIM - Sound\09 SEC.WAV, 0, 2

-1 Cross traffic from left

1048.15, CT, 200, 5, -35, 18, L, *18 35, 0

1048.15, CT, 200, 5, -55, 18, L, *18 35, 0

1148.15, CT, 100, 5, $-35,18, \mathrm{~L}, * 18 \sim 35,0$

1148.15, CT, 100, 5, -55, 18, L, *18 35, 0

1235, CT, 13.15, 5, -40, 18, L, *18 35, 0

1235, CT, 13.15, 5, -85, 18, L, *18 35, 0

1235, CT, 13.15, 5, $-115,18, \mathrm{~L}, * 18 \sim 35,0$

1235, CT, $13.15,5,-135,18, \mathrm{~L}, * 18 \sim 35,0$

1235, CT, 13.15, 5, -152, 18, L, *18 35, 0

1235, CT, $13.15,5,-165,18, \mathrm{~L}, * 18 \sim 35,0$

$1235, \mathrm{CT}, 13.15,5,-175,19, \mathrm{~L}, * 18 \sim 35,0$

-1 Cross traffic from right

951.85, CT, 300, 5, 35, 18, R, *18 35, 0

951.85, CT, 300, 5, 75, 18, R, *18 35, 0

951.85, CT, 300, 5, 95, 18, R, *18 35, 0

951.85, CT, 300, 5, 125, 18, R, *18 35, 0

$1235, \mathrm{CT}, 16.85,5,170,19, \mathrm{R}, * 18 \sim 35,0$

-1 The driver must turn before the arrival of this vehicle 1235, CT, 13.15, 5, -235, 15, L, 3, 1 
-1 Other vehicles from left 1235, CT, 13.15, 5, -255, 17, L, *18 35, 1 1235, CT, 13.15, 5, -295, 17, L, *18 35, 1 1235, CT, 13.15, 5, -345, 17, L, *18 35, 1 1235, CT, 13.15, 5, -405, 17, L, *18 35, 1

-1 Other vehicles from right 1235, CT, $16.85,5,365,17, \mathrm{R}, * 18 \sim 35,1$ 1235, CT, 16.85, 5, 395, 17, R, *18 35, 1 1235, CT, 16.85, 5, 425, 17, R, *18 35, 1 1235, CT, 16.85, 5, 455, 17, R, *18 35, 1 1235, CT, 16.85, 5, 475, 17, R, *18 35, 1

-1 End saving dynamic data

1269, ESAV

-1 Control Vehicle for Intersection 04

1270, CV, 19, 0

1682, CV, 0, 0

1683, BRK, 45, 1, 40

1723, BRK, 72, 2, 0

-1 Start saving dynamic data

1730, BSAV, 1, .4975, THROTTLE 04, 1, 6, 27

-1 intersection 04 (distance $1750 \mathrm{~m}$ from start), full intersection, right turn, Two-way Stop controlled

1410, I, 0, 340, 1, 0, 0

1410, SIGN, 2, 200, 0, 1, 0, 0

1410, SIGN, 7, 334, 2, 0, 0, 0

1600, PR, C:ISTISIMISOUND Intersection right.wav, 0, 10

1739, PR, C:IZAMBDATAIDriver TraininglEssam Input Files\STISIM - Sound 12 SEC.WAV, 0, 2

-1 Cross traffic from left

1548.15, CT, 200, 5, -35, 18, L, *18 35, 0

$1548.15, \mathrm{CT}, 200,5,-55,18, \mathrm{~L}, * 18 \sim 35,0$

1648.15, CT, 100, 5, -35, 18, L, *18 35, 0

1648.15, CT, 100, 5, $-55,18, \mathrm{~L}, * 18 \sim 35,0$

1735, CT, 13.15, 5, -40, 18, L, *18 35, 0

1735, CT, 13.15, 5, -85, 18, L, *18 35, 0 
1735, CT, 13.15, 5, -115, 18, L, *18 35, 0

1735, CT, 13.15, 5, -135, 18, L, *18 35, 0

1735, CT, 13.15, 5, -182, 18, L, *18 35, 0

1735, CT, 13.15, 5, -215, 18, L, *18 35, 0

1735, CT, 13.15, 5, -235, 19, L, *18 35, 0

-1 Cross traffic from right

1451.85, CT, 300, 5, 35, 18, R, *18 35, 0

1451.85, CT, 300, 5, 75, 18, R, *18 35, 0

1451.85, CT, 300, 5, 95, 18, R, *18 35, 0

1451.85, CT, 300, 5, 125, 18, R, *18 35, 0

$1735, \mathrm{CT}, 16.85,5,170,19, \mathrm{R}, * 18 \sim 35,0$

-1 The driver must turn before the arrival of this vehicle

1735, CT, 13.15, 5, -335, 17, L, 3, 1

1735, CT, $16.85,5,345,17, \mathrm{R}, 3,1$

-1 Other vehicles from left

1735, CT, 13.15, 5, -355, 16, L, *18 35, 1

1735, CT, $13.15,5,-395,16, \mathrm{~L}, * 18 \sim 35,1$

1735, CT, $13.15,5,-445,16, \mathrm{~L}, * 18 \sim 35,1$

-1 Other vehicles from right

1735, CT, $16.85,5,375,16, \mathrm{R}, * 18 \sim 35,1$

1735, CT, $16.85,5,395,16, \mathrm{R}, * 18 \sim 35,1$

1735, CT, $16.85,5,425,16, \mathrm{R}, * 18 \sim 35,1$

1735, CT, $16.85,5,455,16, \mathrm{R}, * 18 \sim 35,1$

1735, CT, $16.85,5,475,16, \mathrm{R}, * 18 \sim 35,1$

-1 End saving dynamic data

1769, ESAV

-1 Control Vehicle for Intersection 05

$1770, \mathrm{CV}, 19,0$

2282, CV, 0, 0

2283, BRK, 45, 1, 40

2323, BRK, 70, 2, 0

-1 Start saving dynamic data

2330, BSAV, 1, .4975, THROTTLE 05, 1, 6, 27 
-1 intersection 05 (distance $2350 \mathrm{~m}$ from start), full intersection, left turn, Two-way Stop controlled 2010, I, 0, 340, 1, 0, 0

2010, SIGN, 2, 200, 0, 1, 0, 0

2010, SIGN, 7, 334, 2, 0, 0, 0

2200, PR, C:ISTISIMISOUND Intersection right.wav, 0, 10

2339, PR, C:IZAMBDATAIDriver TraininglEssam Input Files\STISIM - Sound 12 SEC.WAV, 0, 2

-1 Cross traffic from left

2148.15, CT, 200, 5, -35, 18, L, *18 35, 0

2148.15, CT, 200, 5, $-55,18, \mathrm{~L}, * 18 \sim 35,0$

$2248.15, \mathrm{CT}, 100,5,-35,18, \mathrm{~L}, * 18 \sim 35,0$

$2248.15, \mathrm{CT}, 100,5,-55,18, \mathrm{~L}, * 18 \sim 35,0$

2335, CT, 13.15, 5, -40, 18, L, *18 35, 0

2335, CT, $13.15,5,-85,18, \mathrm{~L}, * 18 \sim 35,0$

2335, CT, $13.15,5,-115,18, \mathrm{~L}, * 18 \sim 35,0$

2335, CT, $13.15,5,-145,18, \mathrm{~L}, * 18 \sim 35,0$

2335, CT, $13.15,5,-172,18, \mathrm{~L}, * 18 \sim 35,0$

2335, CT, 13.15, 5, -191, 18, L, *18 35, 0

2335, CT, $13.15,5,-220,18, \mathrm{~L}, * 18 \sim 35,0$

-1 Cross traffic from right

2051.85, CT, 300, 5, 35, 18, R, *18 35, 0

2051.85, CT, 300, 5, 75, 18, R, *18 35, 0

2051.85, CT, 300, 5, 95, 18, R, *18 35, 0

2051.85, CT, 300, 5, 125, 18, R, *18 35, 0

$2335, \mathrm{CT}, 16.85,5,170,18, \mathrm{R}, * 18 \sim 35,0$

-1 The driver must turn before the arrival of these vehicles

2335, CT, 13.15, 5, -375, 16, L, 3, 1

2335, CT, 16.85, 5, 355, 16, R, 3, 1

-1 Other vehicles from left

2335, CT, 13.15, 5, -385, 16, L, *18 35, 1

2335, CT, 13.15, 5, -415, 16, L, *18 35, 1

2335, CT, 13.15, 5, -435, 16, L, *18 35, 1

2335, CT, 13.15, 5, -475, 16, L, *18 35, 1

-1 Other vehicles from right

2335, CT, 16.85, 5, 365, 16, R, *18 35, 1

2335, CT, 16.85, 5, 395, 16, R, *18 35, 1

2335, CT, 16.85, 5, 425, 16, R, *18 35, 1

2335, CT, 16.85, 5, 455, 16, R, *18 35, 1

2335, CT, 16.85, 5, 475, 16, R, *18 35, 1 
-1 End saving dynamic data

2369, ESAV

-1 Adding curve to the roadway

2450, C, 300, 30, 190, 30, .0025, 1, 4

-1 Changing the road to 4-lane Arterial Road

3000, ROAD, 3.7, 4, 2, 2, 0.15, 0.85, .7, .12, .16, 37, -2, -2, -4, 1.83, -4, 1.83, 0, 1.52, 0, 1.52, 0, 0, 0, C:ISTISIM\DatalTextureslGrass08.Jpg, 25, C:ISTISIM\DatalTextures\Road07.Jpg, 12,

C:ISTISIM\DatalTextures\Grass08.Jpg, 25

-1 Vehicles travelling on the same direction of the driver

$3045, \mathrm{~V}, 18,220,5.55,1,21$

$3080, \mathrm{~V}, 17,210,5.55,1,26$

$3140, \mathrm{~V}, 19,210,5.55,1, * 18 \sim 35$

$3180, \mathrm{~V}, 19,230,5.55,1, * 18 \sim 35$

$3380, \mathrm{~V}, 19,230,5.55,1, * 18 \sim 37$

$31080, \mathrm{~V}, 19,185,5.55,1, * 18 \sim 28$

$4180, \mathrm{~V}, 16,195.64,5.55,1, * 18 \sim 28$

$4250, \mathrm{~V}, 20,195.64,5.55,1, * 18 \sim 28$

$4650, \mathrm{~V}, 20,195.64,5.55,1, * 18 \sim 28$

-1 Control Vehicle for Intersection 06

$2370, \mathrm{CV}, 19,0$

$3279, \mathrm{CV}, 0,0$

3280, BRK, 45, 1,40

3320, BRK, 72, 2, 0

-1 Start saving dynamic data

3330, BSAV, 1, .4975, THROTTLE 06, 1, 6, 27

-1 intersection 06 (distance $3350 \mathrm{~m}$ from start), full intersection, going straight, Two-way Stop controlled

$3010, \mathrm{I}, 0,340,1,0,0$

3010, SIGN, 2, 200, 0, 1, 0, 0

3010, SIGN, 7, 334, 2, 0, 0, 0

3200, PR, C:ISTISIMISOUND Intersection straight.wav, 0, 10

3336, PR, C:IZAMBDATAIDriver TraininglEssam Input Files\STISIM - Sound 12 SEC.WAV, 0, 2 
-1 Cross traffic from left (left lane)

3148.15, CT, 200, 5, -35, 18, L, *18 35, 0

3148.15, CT, 200, 5, -55, 18, L, *18 35, 0

3248.15, CT, 100, 5, -35, 18, L, *18 35, 0

3248.15, CT, 100, 5, -55, 18, L, *18 35, 0

$3335, \mathrm{CT}, 13.15,5,-40,18, \mathrm{~L}, * 18 \sim 35,0$

$3335, \mathrm{CT}, 13.15,5,-85,18, \mathrm{~L}, * 18 \sim 35,0$

3335, CT, 13.15, 5, -115, 18, L, *18 35, 0

3335, CT, 13.15, 5, -145, 18, L, *18 35, 0

3335, CT, 13.15, 5, -172, 18, L, *18 35, 0

3335, CT, 13.15, 5, -191, 18, L, *18 35, 0

3335, CT, $13.15,5,-220,18, \mathrm{~L}, * 18 \sim 35,0$

-1 Cross traffic from left (right lane)

3148.15, CT, 196.3, 5, -38, 18, L, *18 35, 0

3148.15, CT, 196.3, 5, -59, 18, L, *18 35, 0

3248.15, CT, 96.3, 5, -32, 18, L, *18 35, 0

3248.15, CT, 96.3, 5, -58, 18, L, *18 35, 0

3335, CT, 9.45, 5, -36, 21, L, *18 35, 0

3335, CT, 9.45, 5, $-88,20, \mathrm{~L}, * 18 \sim 35,0$

3335, CT, 9.45, 5, -105, 18, L, *18 35, 0

3335, CT, 9.45, 5, $-135,18, \mathrm{~L}, * 18 \sim 35,0$

3335, CT, 9.45, 5, -162, 18, L, *18 35, 0

3335, CT, 9.45, 5, -181, 18, L, *18 35, 0

$3335, \mathrm{CT}, 9.45,5,-210,18, \mathrm{~L}, * 18 \sim 35,0$

-1 Cross traffic from right (left lane)

3051.85, CT, 300, 5, 35, 18, R, *18 35, 0

$3051.85, \mathrm{CT}, 300,5,75,18, \mathrm{R}, * 18 \sim 35,0$

$3051.85, \mathrm{CT}, 300,5,95,18, \mathrm{R}, * 18 \sim 35,0$

$3051.85, \mathrm{CT}, 300,5,125,18, \mathrm{R}, * 18 \sim 35,0$

$3335, \mathrm{CT}, 16.85,5,170,18, \mathrm{R}, * 18 \sim 35,0$

-1 Cross traffic from right (right lane)

3051.85, CT, 303.7, 5, 35, 18, R, *18 35, 0

3051.85, CT, 303.7, 5, 75, 18, R, *18 35, 0

3051.85, CT, 303.7, 5, 95, 18, R, *18 35, 0

3051.85, CT, 303.7, 5, 125, 18, R, *18 35, 0

$3335, \mathrm{CT}, 20.55,5,170,18, \mathrm{R}, * 18 \sim 35,0$

-1 The driver must turn before the arrival of these vehicles

3335, CT, 13.15, 5, -335, 17, L, 3, 1

$3335, \mathrm{CT}, 16.85,5,345,17, \mathrm{R}, 3,1$

3335, CT, 9.45, 5, -345, 17, L, 3, 1

3335, CT, 20.55, 5, 355, 17, R, 3, 1 
-1 Other vehicles from left 3335, CT, 13.15, 5, -385, 17, L, *18 35, 1 3335, CT, 13.15, 5, -415, 17, L, *18 35, 1 3335, CT, 13.15, 5, -435, 17, L, *18 35, 1 $3335, \mathrm{CT}, 13.15,5,-475,17, \mathrm{~L}, * 18 \sim 35,1$

-1 Other vehicles from right 3335 , CT, $16.85,5,365,17, \mathrm{R}, * 18 \sim 35,1$ 3335, CT, 16.85, 5, 395, 17, R, *18 35, 1 3335, CT, $16.85,5,425,17, \mathrm{R}, * 18 \sim 35,1$ 3335, CT, $16.85,5,455,17, \mathrm{R}, * 18 \sim 35,1$ $3335, \mathrm{CT}, 16.85,5,475,17, \mathrm{R}, * 18 \sim 35,1$

-1 End saving dynamic data

3369, ESAV

-1 Control Vehicle for Intersection 07

$3370, \mathrm{CV}, 19,0$

$3679, \mathrm{CV}, 0,0$

3680, BRK, 45, 1, 40

3720, BRK, 72, 2, 0

-1 Start saving dynamic data

3730, BSAV, 1, .4975, THROTTLE 07, 1, 6, 27

-1 intersection 07 (distance $3750 \mathrm{~m}$ from start), full intersection, left turn, Two-way stop controlled

$3410, \mathrm{I}, 0,340,1,0,0$

3410, SIGN, 2, 200, 0, 1, 0, 0

3410, SIGN, 7, 334, 2, 0, 0, 0

3600, PR, C:ISTISIM \SOUND Intersection left.wav, 0, 10

3736, PR, C:IZAMBDATAIDriver TraininglEssam Input Files\STISIM - Sound $\backslash 10$ SEC.WAV, 0, 2

-1 Cross traffic from left (left lane)

$3548.15, \mathrm{CT}, 200,5,-35,18, \mathrm{~L}, * 18 \sim 35,0$

$3548.15, \mathrm{CT}, 200,5,-55,18, \mathrm{~L}, * 18 \sim 35,0$

$3648.15, \mathrm{CT}, 100,5,-35,18, \mathrm{~L}, * 18 \sim 35,0$

$3648.15, \mathrm{CT}, 100,5,-55,18, \mathrm{~L}, * 18 \sim 35,0$

3735, CT, 13.15, 5, -40, 18, L, *18 35, 0

3735, CT, $13.15,5,-85,18, \mathrm{~L}, * 18 \sim 35,0$

3735, CT, 13.15, 5, -115, 18, L, *18 35, 0 
3735, CT, 13.15, 5, -135, 18, L, *18 35, 0

3735, CT, 13.15, 5, -152, 18, L, *18 35, 0

3735, CT, 13.15, 5, -171, 18, L, *18 35, 0

3735, CT, 13.15, 5, -195, 19, L, *18 35, 0

-1 Cross traffic from left (right lane)

3548.15, CT, 196.3, 5, -45, 18, L, *18 35, 0

3548.15, CT, 196.3, 5, -65, 18, L, *18 35, 0

3648.15, CT, 96.3, 5, -40, 18, L, *18 35, 0

3648.15, CT, 96.3, 5, -62, 18, L, *18 35, 0

3735, CT, 9.45, 5, -45, 18, L, *18 35, 0

3735, CT, 9.45, 5, -88, 18, L, *18 35, 0

3735, CT, 9.45, 5, -121, 18, L, *18 35, 0

3735, CT, 9.45, 5, -141, 18, L, *18 35, 0

3735, CT, 9.45, 5, -155, 18, L, *18 35, 0

3735, CT, 9.45, 5, -175, 18, L, *18 35, 0

3735, CT, 9.45, 5, -198, 19, L, *18 35, 0

-1 Cross traffic from right (left lane)

$3451.85, \mathrm{CT}, 300,5,35,18, \mathrm{R}, * 18 \sim 35,0$

3451.85, CT, 300, 5, 75, 18, R, *18 35, 0

3451.85 , CT, 300, 5, 95, 18, R, *18 35, 0

$3451.85, \mathrm{CT}, 300,5,125,18, \mathrm{R}, * 18 \sim 35,0$

$3735, \mathrm{CT}, 16.85,5,185,19, \mathrm{R}, * 18 \sim 35,0$

-1 Cross traffic from right (right lane)

3451.85, CT, 303.7, 5, 35, 18, R, *18 35, 0

3451.85, CT, 303.7, 5, 75, 18, R, *18 35, 0

3451.85, CT, 303.7, 5, 95, 18, R, *18 35, 0

3451.85, CT, 303.7, 5, 125, 18, R, *18 35, 0

$3735, \mathrm{CT}, 20.55,5,180,19, \mathrm{R}, * 18 \sim 35,0$

-1 The driver must turn before the arrival of these vehicles

3735, CT, 13.15, 5, -285, 16, L, 3, 1

3735, CT, 9.45, 5, -295, 16, L, 3, 1

3735, CT, 16.85, 5, 295, 16, R, 3, 1

3735, CT, 20.55, 5, 298, 16, R, 3, 1

-1 Other vehicles from left (left lane)

3735, CT, 13.15, 5, -295, 16, L, *18 35, 1

$3735, \mathrm{CT}, 13.15,5,-315,16, \mathrm{~L}, * 18 \sim 35,1$

$3735, \mathrm{CT}, 13.15,5,-345,16, \mathrm{~L}, * 18 \sim 35,1$

$3735, \mathrm{CT}, 13.15,5,-395,16, \mathrm{~L}, * 18 \sim 35,1$

3735, CT, $13.15,5,-415,16, \mathrm{~L}, * 18 \sim 35,1$

3735, CT, 13.15, 5, -445, 16, L, *18 35, 1 
-1 Other vehicles from left (right lane) 3735, CT, 9.45, 5, -305, 16, L, *18 35, 1 3735, CT, 9.45, 5, -325, 16, L, *18 35, 1 3735, CT, 9.45, 5, -355, 16, L, * 18 35, 1 3735, CT, 9.45, 5, -400, 16, L, *18 35, 1 3735, CT, 9.45, 5, -418, 16, L, *18 35, 1 3735, CT, 9.45, 5, -449, 16, L, * 18 35, 1

-1 Other vehicles from right (left lane) 3735, CT, 16.85, 5, 325, 17, R, *18 35, 1 3735, CT, 16.85, 5, 395, 17, R, *18 35, 1 3735, CT, 16.85, 5, 425, 17, R, *18 35, 1 $3735, \mathrm{CT}, 16.85,5,455,17, \mathrm{R}, * 18 \sim 35,1$

-1 Other vehicles from right (right lane) 3735, CT, 20.55, 5, 338, 17, R, *18 35, 1 3735, CT, 20.55, 5, 398, 17, R, *18 35, 1 3735, CT, 20.55, 5, 427, 17, R, *18 35, 1 3735, CT, 20.55, 5, 465, 17, R, *18 35, 1

-1 End saving dynamic data

3769, ESAV

-1 Control Vehicle for Intersection 08

3770, CV, 19, 0

4179, CV, 0, 0

4180, BRK, 45, 1, 40

4220, BRK, 72, 2, 0

-1 Start saving dynamic data

4230, BSAV, 1, .4975, THROTTLE 08, 1, 6, 27

-1 intersection 08 (distance $4250 \mathrm{~m}$ from start), full intersection, going straight, Two-way stop controlled

3910, I, 0, 340, 1, 0, 0

3910, SIGN, 2, 200, 0, 1, 0, 0

3910, SIGN, 7, 334, 2, 0, 0, 0

4100, PR, C:ISTISIMISOUND\Intersection straight.wav, 0, 10

4236, PR, C:IZAMBDATAIDriver TrainingIEssam Input FilesISTISIM - Sound\08 SEC.WAV, 0, 2 
-1 Cross traffic from left (left lane)

4048.15, CT, 200, 5, -35, 18, L, *18 35, 0

4048.15, CT, 200, 5, -55, 18, L, *18 35, 0

4148.15, CT, 100, 5, -35, 18, L, *18 35, 0

4148.15, CT, 100, 5, -55, 18, L, *18 35, 0

4235, CT, 13.15, 5, -65, 18, L, *18 35, 0

4235, CT, 13.15, 5, -80, 18, L, *18 35, 0

4235, CT, 13.15, 5, -98, 18, L, *18 35, 0

4235, CT, 13.15, 5, -117, 18, L, *18 35, 0

4235, CT, 13.15, 5, -141, 19, L, *18 35, 0

-1 Cross traffic from left (right lane)

4048.15, CT, 196.3, 5, -45, 18, L, *18 35, 0

4048.15, CT, 196.3, 5, -65, 18, L, *18 35, 0

4148.15, CT, 96.3, 5, -40, 18, L, *18 35, 0

4148.15, CT, 96.3, 5, -62, 18, L, *18 35, 0

4235, CT, 9.45, 5, -45, 18, L, *18 35, 0

4235, CT, 9.45, 5, $-88,18, \mathrm{~L}, * 18 \sim 35,0$

4235, CT, 9.45, 5, -101, 18, L, *18 35, 0

4235, CT, 9.45, 5, -121, 18, L, *18 35, 0

4235, CT, 9.45, 5, -144, 19, L, *18 35, 0

-1 Cross traffic from right (left lane)

3951.85, CT, 300, 5, 35, 18, R, *18 35, 0

$3951.85, \mathrm{CT}, 300,5,55,18, \mathrm{R}, * 18 \sim 35,0$

3951.85, CT, 300, 5, 70, 18, R, *18 35, 0

4235, CT, 16.85, 5, 131, 19, R, *18 35, 0

-1 Cross traffic from right (right lane)

3951.85, CT, 303.7, 5, 35, 18, R, *18 35, 0

3951.85, CT, 303.7, 5, 75, 18, R, *18 35, 0

3951.85, CT, 303.7, 5, 95, 18, R, *18 35, 0

4235, CT, 20.55, 5, 125, 19, R, *18 35, 0

-1 The driver must turn before the arrival of these vehicles

4235, CT, 13.15, 5, -235, 16, L, 3, 1

4235, CT, 9.45, 5, -240, 16, L, 3, 1

4235, CT, 16.85, 5, 240, 16, R, 3, 1

4235, CT, 20.55, 5, 243, 16, R, 3, 1

-1 Other vehicles from left (left lane)

4235, CT, 13.15, 5, -245, 16, L, *18 35, 1

4235, CT, 13.15, 5, -305, 16, L, *18 35, 1

4235, CT, $13.15,5,-345,16, \mathrm{~L}, * 18 \sim 35,1$

4235, CT, 13.15, 5, -395, 16, L, *18 35, 1 
4235, CT, 13.15, 5, -415, 16, L, *18 35, 1

4235, CT, 13.15, 5, -445, 16, L, *18 35, 1

-1 Other vehicles from left (right lane) 4235, CT, 9.45, 5, -285, 16, L, * 18 35, 1 4235, CT, 9.45, 5, -315, 16, L, *18 35, 1 4235, CT, 9.45, 5, -355, 16, L, *18 35, 1 4235, CT, 9.45, 5, -400, 16, L, *18 35, 1 4235, CT, 9.45, 5, -418, 16, L, * 18 35, 1 4235, CT, 9.45, 5, -449, 16, L, *18 35, 1

-1 Other vehicles from right (left lane) 4235, CT, 16.85, 5, 255, 17, R, *18 35, 1 4235, CT, $16.85,5,285,17, \mathrm{R}, * 18 \sim 35,1$ 4235, CT, $16.85,5,325,17, \mathrm{R}, * 18 \sim 35,1$ 4235, CT, 16.85, 5, 355, 17, R, *18 35, 1

-1 Other vehicles from right (right lane) 4235, CT, 20.55, 5, 260, 17, R, *18 35, 1 4235, CT, 20.55, 5, 298, 17, R, *18 35, 1 4235, CT, 20.55, 5, 327, 17, R, *18 35, 1 4235, CT, 20.55, 5, 365, 17, R, *18 35, 1

-1 End saving dynamic data

4269, ESAV

-1 Control Vehicle for Intersection 09

4270, CV, 19, 0

$4679, \mathrm{CV}, 0,0$

4680, BRK, 45, 1, 40

$4720, \mathrm{BRK}, 72,2,0$

-1 Start saving dynamic data

4730, BSAV, 1, .4975, THROTTLE 09, 1, 6, 27

-1 intersection 09 (distance $4750 \mathrm{~m}$ from start), full intersection, left turn, Two-way stop controlled 4410, I, 0, 340, 1, 0, 0 4410, SIGN, 2, 200, 0, 1, 0, 0 4410, SIGN, 7, 334, 2, 0, 0, 0 
4600, PR, C:ISTISIM\SOUND Intersection left.wav, 0, 10

4736, PR, C:IZAMBDATAIDriver TraininglEssam Input Files\STISIM - Sound\06 SEC.WAV, 0, 2

-1 Cross traffic from left (left lane)

4548.15, CT, 200, 5, -35, 18, L, *18 35, 0

4548.15, CT, 200, 5, $-55,18, \mathrm{~L}, * 18 \sim 35,0$

4648.15, CT, 100, 5, -35, 18, L, *18 35, 0

$4648.15, \mathrm{CT}, 100,5,-55,18, \mathrm{~L}, * 18 \sim 35,0$

$4735, \mathrm{CT}, 13.15,5,-65,18, \mathrm{~L}, * 18 \sim 35,0$

4735, CT, $13.15,5,-80,18, \mathrm{~L}, * 18 \sim 35,0$

4735, CT, $13.15,5,-97,18, \mathrm{~L}, * 18 \sim 35,0$

4735, CT, 13.15, 5, -105, 19, L, *18 35, 0

-1 Cross traffic from left (right lane)

4548.15, CT, 196.3, 5, -45, 18, L, *18 35, 0

4548.15, CT, 196.3, 5, -65, 18, L, *18 35, 0

4648.15, CT, 96.3, 5, -40, 18, L, *18 35, 0

4648.15, CT, 96.3, 5, -62, 18, L, *18 35, 0

4735, CT, 9.45, 5, -45, 18, L, *18 35, 0

4735, CT, 9.45, 5, -88, 18, L, *18 35, 0

4735, CT, 9.45, 5, $-98,18, \mathrm{~L}, * 18 \sim 35,0$

4735, CT, 9.45, 5, -108, 19, L, *18 35, 0

-1 Cross traffic from right (left lane)

4451.85, CT, 300, 5, 35, 18, R, *18 35, 0

4451.85, CT, 300, 5, 55, 18, R, *18 35, 0

4451.85, CT, 300, 5, 70, 18, R, *18 35, 0

4735, CT, $16.85,5,81,19, \mathrm{R}, * 18 \sim 35,0$

4735, CT, 16.85, 5, 101, 19, R, *18 35, 0

-1 Cross traffic from right (right lane)

4451.85, CT, 303.7, 5, 35, 18, R, *18 35, 0

4451.85, CT, 303.7, 5, 75, 18, R, *18 35, 0

4451.85, CT, 303.7, 5, 95, 18, R, *18 35, 0

4735, CT, 20.55, 5, 25, 19, R, *18 35, 0

4735, CT, 20.55, 5, 45, 19, R, *18 35, 0

4735, CT, 20.55, 5, 75, 19, R, *18 35, 0

4735, CT, 20.55, 5, 95, 19, R, *18 35, 0

-1 The driver must turn before the arrival of these vehicles

4735, CT, 13.15, 5, -185, 16, L, 3, 1

4735, CT, 9.45, 5, -190, 16, L, 3, 1

4735, CT, 13.15, 5, 185, 16, R, 3, 1 
-1 Other vehicles from left (left lane) 4735, CT, 13.15, 5, -225, 16, L, *18 35, 1 4735, CT, 13.15, 5, -275, 16, L, *18 35, 1 4735, CT, 13.15, 5, -325, 16, L, *18 35, 1 4735, CT, 13.15, 5, -375, 16, L, *18 35, 1 4735, CT, 13.15, 5, -415, 16, L, *18 35, 1 4735, CT, 13.15, 5, -445, 16, L, *18 35, 1

-1 Other vehicles from left (right lane) 4735, CT, 9.45, 5, -285, 16, L, *18 35, 1 4735, CT, 9.45, 5, -315, 16, L, *18 35, 1 4735, CT, 9.45, 5, -355, 16, L, *18 35, 1 4735, CT, 9.45, 5, -400, 16, L, *18 35, 1 4735, CT, 9.45, 5, -418, 16, L, *18 35, 1 4735, CT, 9.45, 5, -449, 16, L, *18 35, 1

-1 Other vehicles from right (left lane) 4735, CT, $16.85,5,255,17, \mathrm{R}, * 18 \sim 35,1$ 4735, CT, $16.85,5,285,17, \mathrm{R}, * 18 \sim 35,1$ 4735, CT, $16.85,5,325,17, \mathrm{R}, * 18 \sim 35,1$ $4735, \mathrm{CT}, 16.85,5,355,17, \mathrm{R}, * 18 \sim 35,1$

-1 Other vehicles from right (right lane) 47235, CT, 20.55, 5, 260, 17, R, *18 35, 1 4735, CT, 20.55, 5, 298, 17, R, *18 35, 1 4735, CT, 20.55, 5, 327, 17, R, *18 35, 1 4735, CT, 20.55, 5, 365, 17, R, *18 35, 1

-1 End saving dynamic data

4769, ESAV

-1 Control Vehicle to the end of the simulation 4770, CV, 21, 0

-1 Trees

0, TREE, 90, $0, * 1 \sim 18,9.45,18.29,0$ 335, TREE, $0,0, * 1 \sim 18,9.45,18.29,0$

360, TREE, 90, 0, *1 18, 9.45, 18.29, 0 
735, TREE, $0,0, * 1 \sim 18,9.45,18.29,0$

760, TREE, 90, 0, *1 18, 9.45, 18.29, 0 1235, TREE, $0,0, * 1 \sim 18,9.45,18.29,0$

1260, TREE, 90, $0, * 1 \sim 18,9.45,18.29,0$ 1735, TREE, $0,0, * 1 \sim 18,9.45,18.29,0$

1760, TREE, 90, $0, * 1 \sim 18,9.45,18.29,0$ 2335, TREE, $0,0, * 1 \sim 18,9.45,18.29,0$

2360, TREE, 90, 0, *1 18, 9.45, 18.29, 0 3335, TREE, $0,0, * 1 \sim 18,9.45,18.29,0$

3360, TREE, 90, 0, *1 18, 9.45, 18.29, 0 3735, TREE, $0,0, * 1 \sim 18,9.45,18.29,0$

3760, TREE, 90, 0, *1 18, 9.45, 18.29, 0 4235, TREE, $0,0, * 1 \sim 18,9.45,18.29,0$

4260, TREE, 90, 0, *1 18, 9.45, 18.29, 0 4735, TREE, $0,0, * 1 \sim 18,9.45,18.29,0$

4760, TREE, 90, 0, *1 18, 9.45, 18.29, 0

-1 Buildings

0, BLDG, 250, -14.59, $\mathrm{H}^{* 2 ; 4 \sim 7 ; 9 ; 13}$

0, BLDG, 320, -14.59, $\mathrm{H}^{*} 2 ; 4 \sim 7 ; 9 ; 13$

0, BLDG, 220, 14.59, $\mathrm{H}^{* 2 ; 4 ~ 7 ; 9 ; 13}$

0, BLDG, 300, 14.59, $\mathrm{H}^{* 2 ; 4 ~ 7 ; 9 ; 13}$

360, BLDG, $250,-14.59, \mathrm{H}^{*} 2 ; 4 \sim 7 ; 9 ; 13$

360, BLDG, 320, -14.59, $\mathrm{H}^{* 2 ; 4 ~ 7 ; 9 ; 13}$

360, BLDG, 220, 14.59, H*2;4 7;9;13

360, BLDG, 300, 14.59, $\mathrm{H}^{*} 2 ; 4 \sim 7 ; 9 ; 13$

760, BLDG, 250, -14.59, $\mathrm{H}^{*} 2 ; 4 \sim 7 ; 9 ; 13$

760, BLDG, 320, $-14.59, \mathrm{H}^{* 2 ; 4 \sim 7 ; 9 ; 13}$

760, BLDG, 220, 14.59, $\mathrm{H}^{*} 2 ; 4 \sim 7 ; 9 ; 13$

760, BLDG, 300, 14.59, H*2;4 7;9;13

3154.36, BLDG, 242.02, 19.99, G*1 13

3154.36, BLDG, 282.02, 19.99, $\mathrm{B} * 1 \sim 5 ; 9 ; 13 \sim 15$

3154.36, BLDG, 322.02, 19.99, B*1 5;9;13 15 
3154.36, BLDG, 372.02, 19.99, B*1 5;9;13 15

3154.36, BLDG, 422.02, 19.99, B*1 5;9;13 15

3154.36, BLDG, 462.02, 19.99, G*1 13

3154.36, BLDG, 662.02, 19.99, B*1 5;9;13 15

3154.36, BLDG, 722.02, 19.99, G*1 13

3154.36, BLDG, 812.02, 19.99, B*1 5;9;13 15

3154.36, BLDG, 892.02, 19.99, G*1 13

3154.36, BLDG, 247.02, -19.99, B*1 5;9;13 15

3154.36, BLDG, 285.02, -19.99, G*1 13

3154.36, BLDG, 326.02, -19.99, G*1 13

3154.36, BLDG, 372.02, -19.99, G*1 13

3154.36, BLDG, 432.02, -19.99, B*1 5;9;13 15

3154.36, BLDG, 662.02, -19.99, G*1 13

3154.36, BLDG, 722.02, -19.99, G*1 13

3154.36, BLDG, 812.02, -19.99, G*1 13

3154.36, BLDG, 892.02, -19.99, G*1 13

3355, BLDG, 129.56, -18.29, H*2;4 7;9;13

3355, BLDG, 159.56, -18.29, $\mathrm{H}^{*} 2 ; 4 \sim 7 ; 9 ; 13$

3355, BLDG, 199.56, -18.29, $\mathrm{H}^{*} 2 ; 4 \sim 7 ; 9 ; 13$

3355, BLDG, 239.56, -18.29, $\mathrm{H}^{*} 2 ; 4 \sim 7 ; 9 ; 13$

3355, BLDG, 269.56, -18.29, H*2;4 7;9;13

3355, BLDG, 129.56, 18.29, H*2;4 7;9;13

3355, BLDG, 169.56, 18.29, $\mathrm{H}^{*} 2 ; 4 \sim 7 ; 9 ; 13$

3355, BLDG, 199.56, 18.29, $\mathrm{H}^{*} 2 ; 4 \sim 7 ; 9 ; 13$

3355, BLDG, 249.56, 18.29, H*2;4 7;9;13

3355, BLDG, 279.56, 18.29, $\mathrm{H}^{*} 2 ; 4 \sim 7 ; 9 ; 13$

3755, BLDG, 129.56, -18.29, $\mathrm{H}^{*} 2 ; 4 \sim 7 ; 9 ; 13$ 3755, BLDG, 159.56, -18.29, $\mathrm{H}^{*} 2 ; 4 \sim 7 ; 9 ; 13$ 3755, BLDG, 199.56, -18.29, H*2;4 7;9;13 3755, BLDG, 239.56, -18.29, $\mathrm{H}^{*} 2 ; 4 \sim 7 ; 9 ; 13$ 3755, BLDG, 269.56, -18.29, $\mathrm{H}^{*} 2 ; 4 \sim 7 ; 9 ; 13$ 3755, BLDG, 339.56, -18.29, H*2;4 7;9;13 3755, BLDG, 379.56, -18.29, H*2;4 7;9;13 3755, BLDG, 439.56, -18.29, H*2;4 7;9;13

3755, BLDG, 129.56, 18.29, $\mathrm{H}^{*} 2 ; 4 \sim 7 ; 9 ; 13$ 3755, BLDG, 169.56, 18.29, $\mathrm{H}^{*} 2 ; 4 \sim 7 ; 9 ; 13$ 3755, BLDG, 199.56, 18.29, $\mathrm{H}^{*} 2 ; 4 \sim 7 ; 9 ; 13$ 3755, BLDG, 249.56, 18.29, $\mathrm{H}^{*} 2 ; 4 \sim 7 ; 9 ; 13$ 3755, BLDG, 279.56, 18.29, $\mathrm{H}^{*} 2 ; 4 \sim 7 ; 9 ; 13$ 3755, BLDG, 339.56, 18.29, H*2;4 7;9;13 
3755, BLDG, 389.56, 18.29, $\mathrm{H}^{*} 2 ; 4 \sim 7 ; 9 ; 13$

3755, BLDG, 439.56, 18.29, $\mathrm{H}^{*} 2 ; 4 \sim 7 ; 9 ; 13$

4255, BLDG, 129.56, -18.29, $\mathrm{H}^{* 2 ; 4 ~ 7 ; 9 ; 13 ~}$

4255, BLDG, 159.56, -18.29, $\mathrm{H}^{* 2 ; 4 ~ 7 ; 9 ; 13}$

4255, BLDG, 199.56, -18.29, $\mathrm{H}^{*} 2 ; 4 \sim 7 ; 9 ; 13$

4255, BLDG, 239.56, -18.29, $\mathrm{H}^{*} 2 ; 4 \sim 7 ; 9 ; 13$

4255, BLDG, 269.56, -18.29, $\mathrm{H}^{*} 2 ; 4 \sim 7 ; 9 ; 13$

4255, BLDG, 339.56, -18.29, $\mathrm{H}^{* 2 ; 4 ~ 7 ; 9 ; 13}$

4255, BLDG, 389.56, -18.29, $\mathrm{H}^{*} 2 ; 4 \sim 7 ; 9 ; 13$

4255, BLDG, 429.56, -18.29, H*2;4 7;9;13

4255, BLDG, 129.56, 18.29, H*2;4 7;9;13

4255, BLDG, 169.56, 18.29, $\mathrm{H}^{*} 2 ; 4 \sim 7 ; 9 ; 13$

4255, BLDG, 199.56, 18.29, $\mathrm{H}^{*} 2 ; 4 \sim 7 ; 9 ; 13$

4255, BLDG, 249.56, 18.29, $\mathrm{H}^{*} 2 ; 4 \sim 7 ; 9 ; 13$

4255, BLDG, 279.56, 18.29, $\mathrm{H}^{*} 2 ; 4 \sim 7 ; 9 ; 13$

4255, BLDG, 339.56, 18.29, $\mathrm{H}^{*} 2 ; 4 \sim 7 ; 9 ; 13$

4255, BLDG, 379.56, 18.29, $\mathrm{H}^{* 2 ; 44 ~ 7 ; 9 ; 13}$

4255, BLDG, 419.56, 18.29, $\mathrm{H}^{* 2 ; 4} 7 ; 9 ; 13$

4755, BLDG, 129.56, -18.29, H*2;4 7;9;13

4755, BLDG, 159.56, -18.29, $\mathrm{H}^{*} 2 ; 4 \sim 7 ; 9 ; 13$

4755, BLDG, 199.56, $-18.29, \mathrm{H}^{*} 2 ; 4 \sim 7 ; 9 ; 13$

4755, BLDG, 239.56, -18.29, $\mathrm{H}^{*} 2 ; 4 \sim 7 ; 9 ; 13$

4755, BLDG, 269.56, -18.29, $\mathrm{H}^{*} 2 ; 4 \sim 7 ; 9 ; 13$

4755, BLDG, 339.56, -18.29, $\mathrm{H}^{* 2 ; 4 ~ 7 ; 9 ; 13}$

4755, BLDG, 389.56, -18.29, $\mathrm{H}^{*} 2 ; 4 \sim 7 ; 9 ; 13$

4755, BLDG, 429.56, -18.29, H*2;4 7;9;13

4755, BLDG, 129.56, 18.29, $\mathrm{H}^{*} 2 ; 4 \sim 7 ; 9 ; 13$

4755, BLDG, 169.56, 18.29, H*2;4 7;9;13

4755, BLDG, 199.56, 18.29, H*2;4 7;9;13

4755, BLDG, 249.56, 18.29, H*2;4 7;9;13

4755, BLDG, 279.56, 18.29, H*2;4 7;9;13

4755, BLDG, 339.56, 18.29, H*2;4 7;9;13

4755, BLDG, 379.56, 18.29, $\mathrm{H}^{*} 2 ; 4 \sim 7 ; 9 ; 13$

4755, BLDG, 419.56, 18.29, $\mathrm{H}^{*} 2 ; 4 \sim 7 ; 9 ; 13$

4755, BLDG, 479.56, 18.29, $\mathrm{H}^{*} 2 ; 4 \sim 7 ; 9 ; 13$

4755, BLDG, 499.56, 18.29, H*2;4 7;9;13

4780, ES 


\section{Appendix " $C$ "}

MATLAB Code for Validating the Proposed System 
$\%$ MATLAB code to validate TWSC intersections

$\%$ by: Essam Dabbour

clc

clear all

\%Input vehicle data

POL=input('Input the model of acceleration used for host vehicle (Constant / Linear decay) [L]: ','s');

if isempty(POL)

$\mathrm{POL}=$ 'L';

end

if $\mathrm{POL}==\mathrm{C}^{\prime}$

$\mathrm{AV}=$ input('Input the maximum acceleration provided by the host vehicle in $\mathrm{m} / \mathrm{sec} / \mathrm{sec}$ [2.75]: ');

if isempty(AV)

$\mathrm{AV}=2.75$

end

end

if $\mathrm{POL}=={ }^{\prime} \mathrm{L} '$

$\mathrm{AV}=$ input('Input the maximum initial acceleration provided by the host vehicle in $\mathrm{m} / \mathrm{sec} / \mathrm{sec}$ [3.75]: ');

if isempty(AV)

$\mathrm{AV}=3.75$

end

$\mathrm{EQ}=$ input('Input the equilibrium (crawl) speed of the host vehicle in $\mathrm{m} / \mathrm{sec}$ [40]: ');

if isempty(EQ)

$\mathrm{EQ}=40$;

end

end

$\mathrm{L}=$ input('Input the length of the host vehicle in meters [5.25 m]: ');

if isempty(L)

$\mathrm{L}=5.25$;

end

\%Input driver data

AGE=input('Input the age of the driver in years [28]: ');

if isempty(AGE)

$$
\mathrm{AGE}=28 \text {; }
$$

end

GENDER=input('Input the gender of the driver M/F [M]: ','s');

if isempty(GENDER)

GENDER = 'M';

end

if GENDER $==$ ' $\mathrm{M}$ '

GGENDER=0;

end

if GENDER $==$ ' $\mathrm{F}$ '

GGENDER $=1$;

end

TURN=input('Input driver turning signal $\mathrm{L} / \mathrm{R}$ or "N" for no signal [N]: ','s');

if isempty(TURN) 


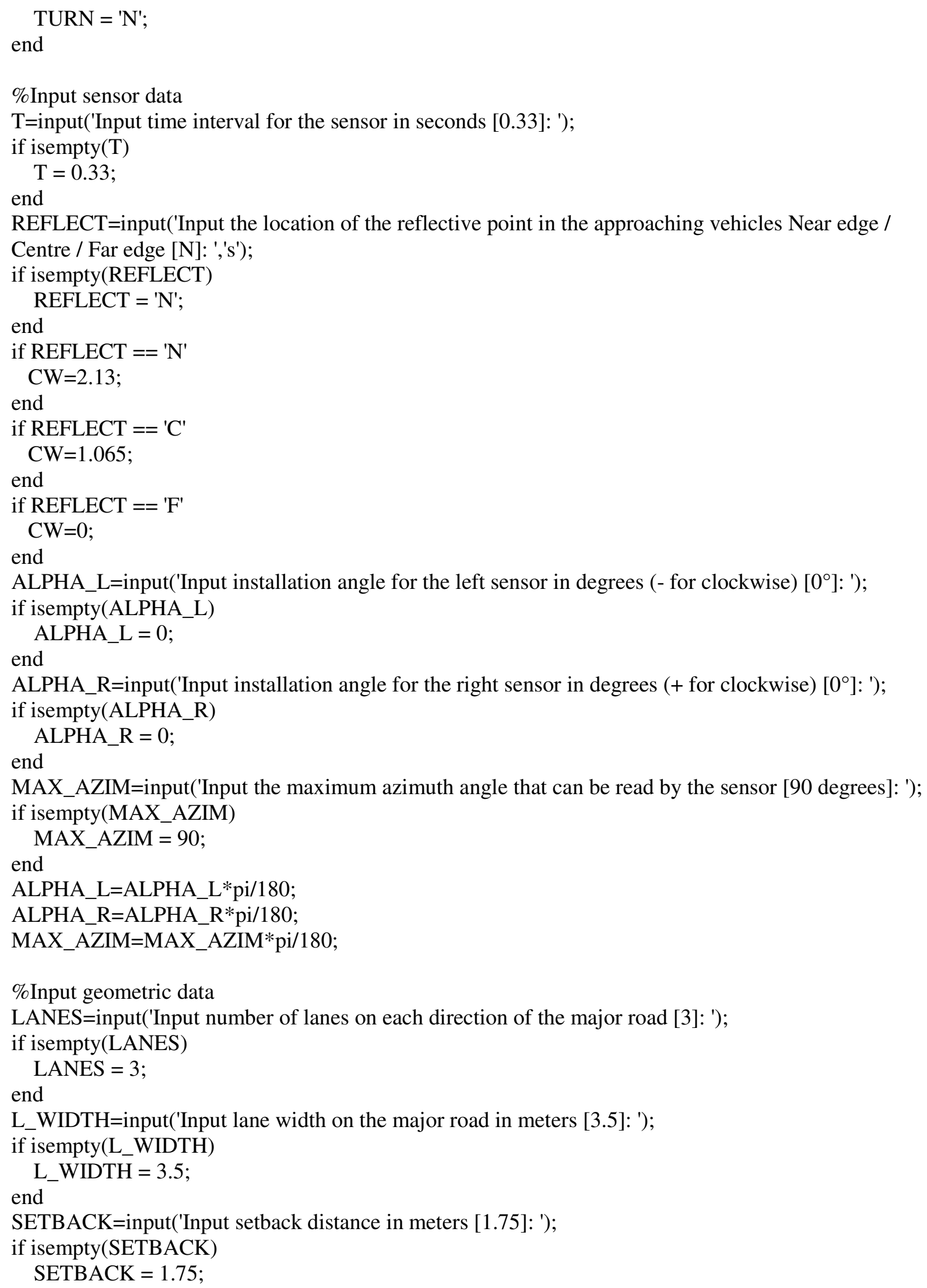




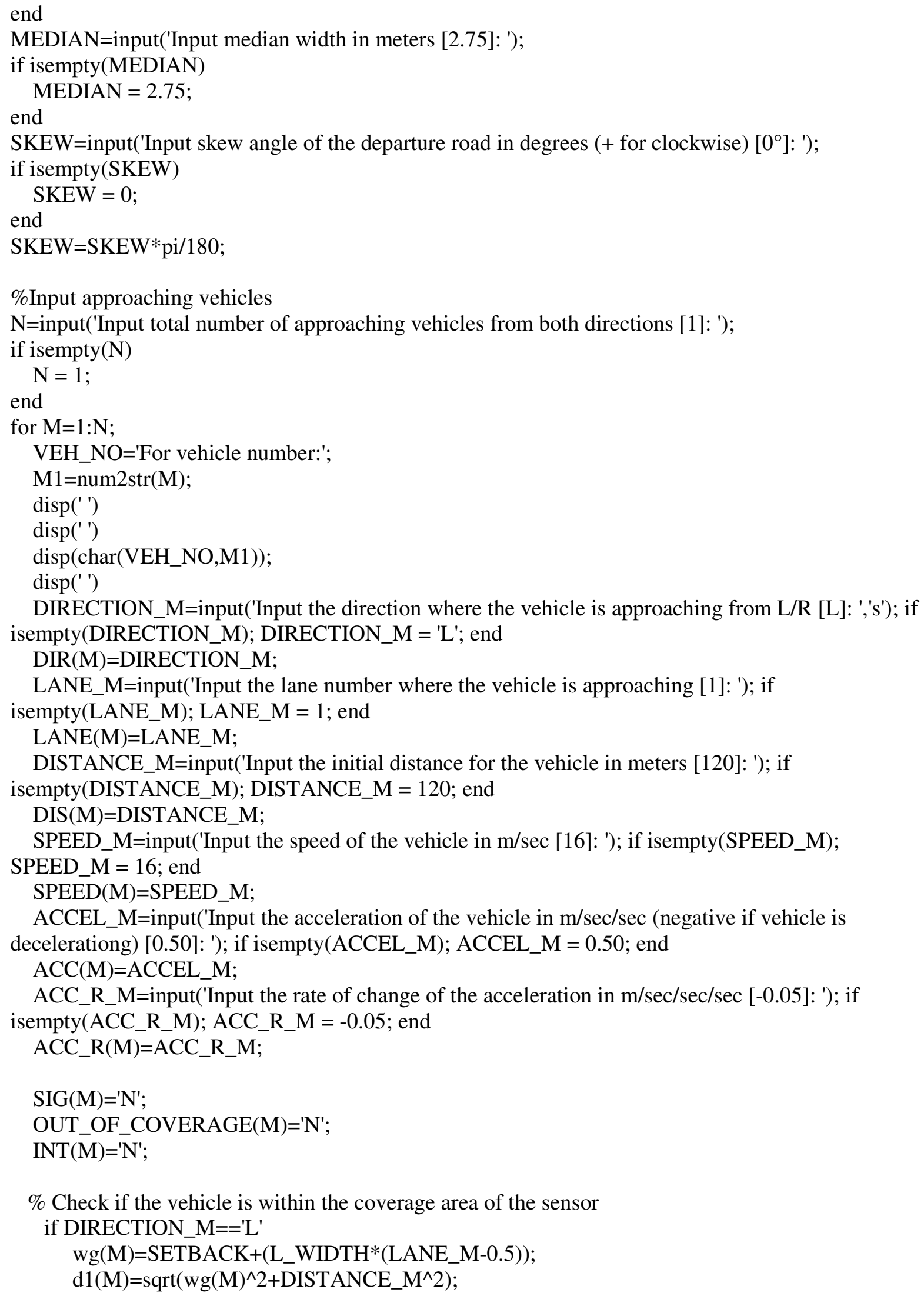




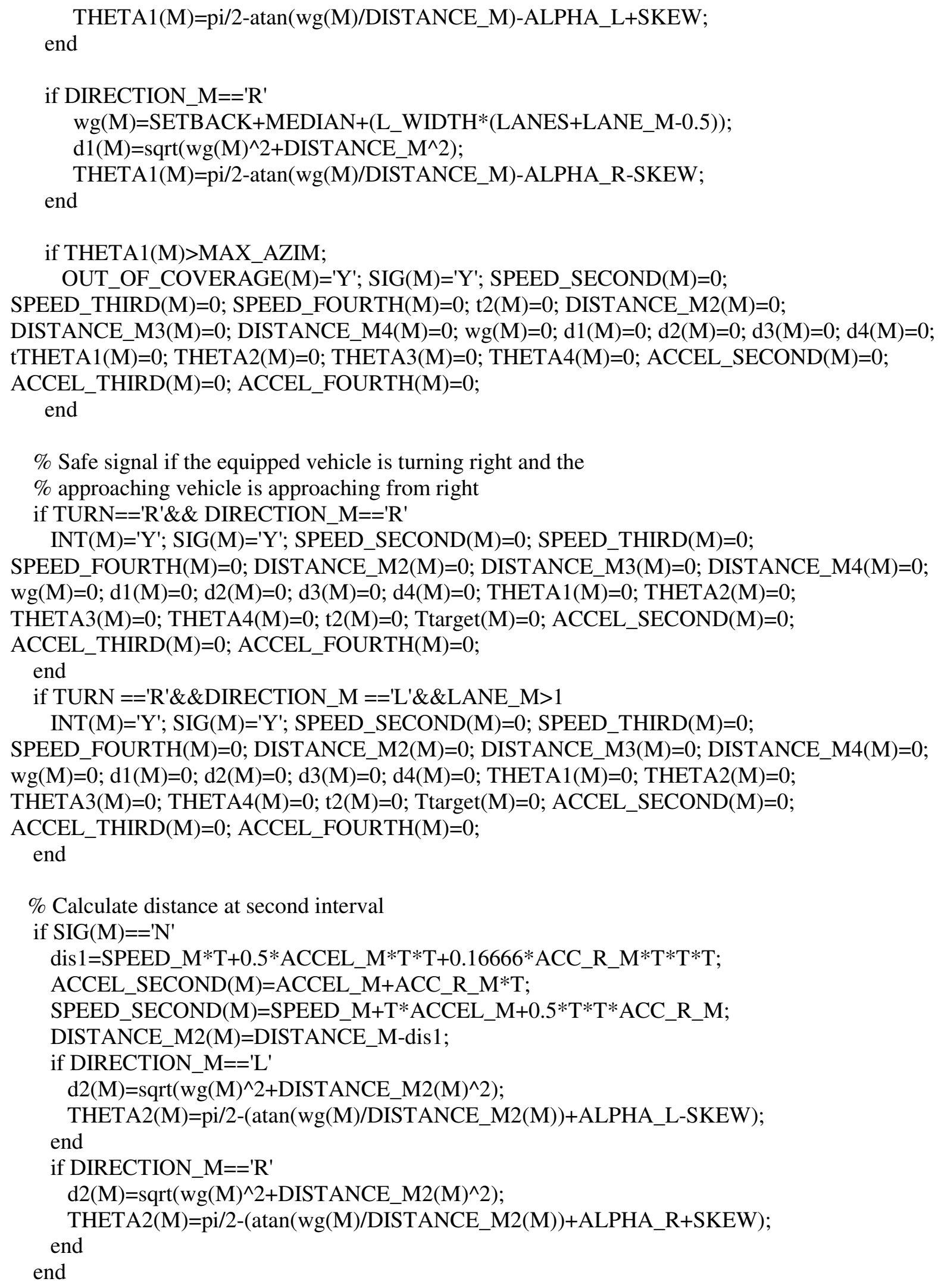




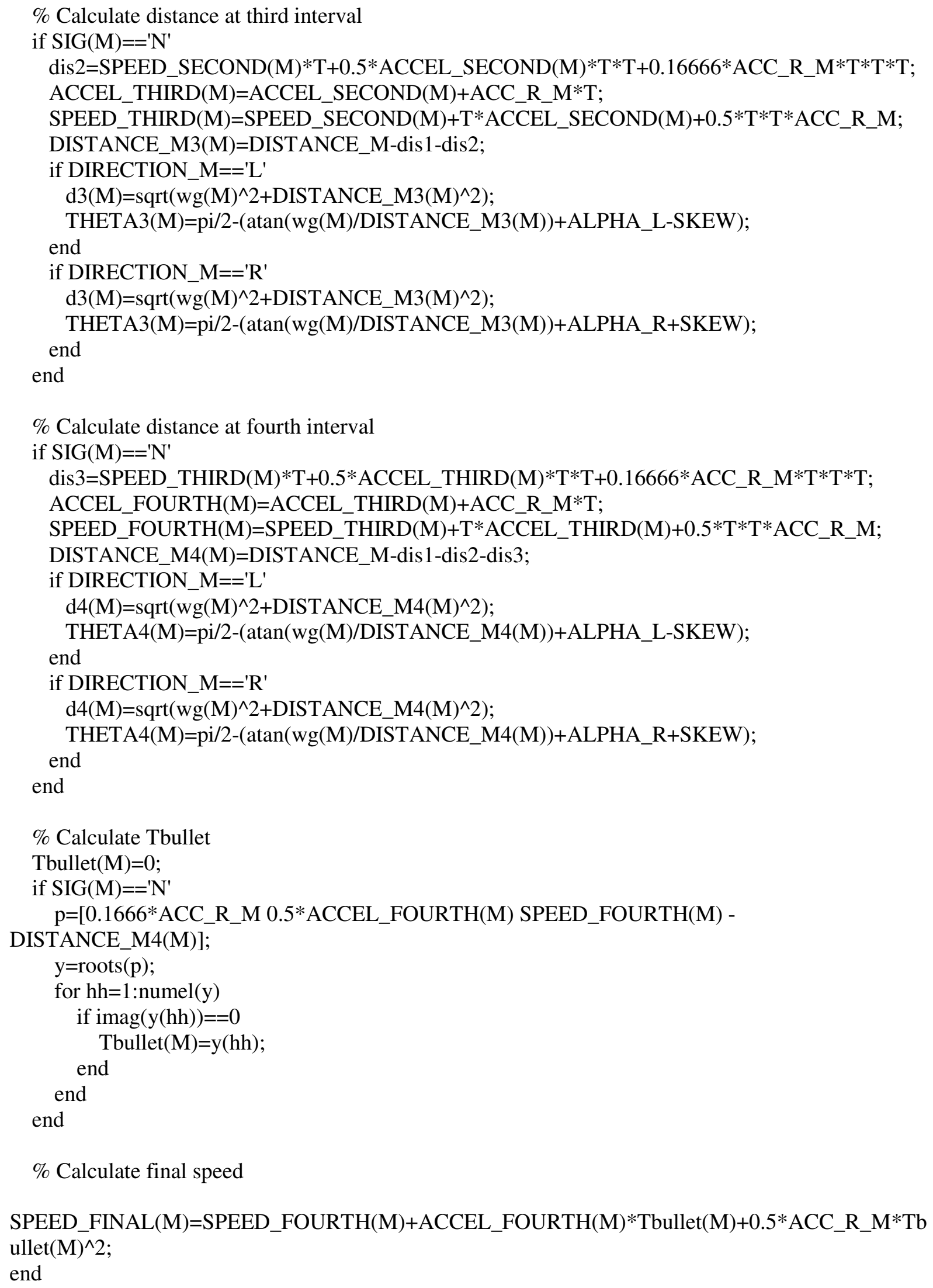




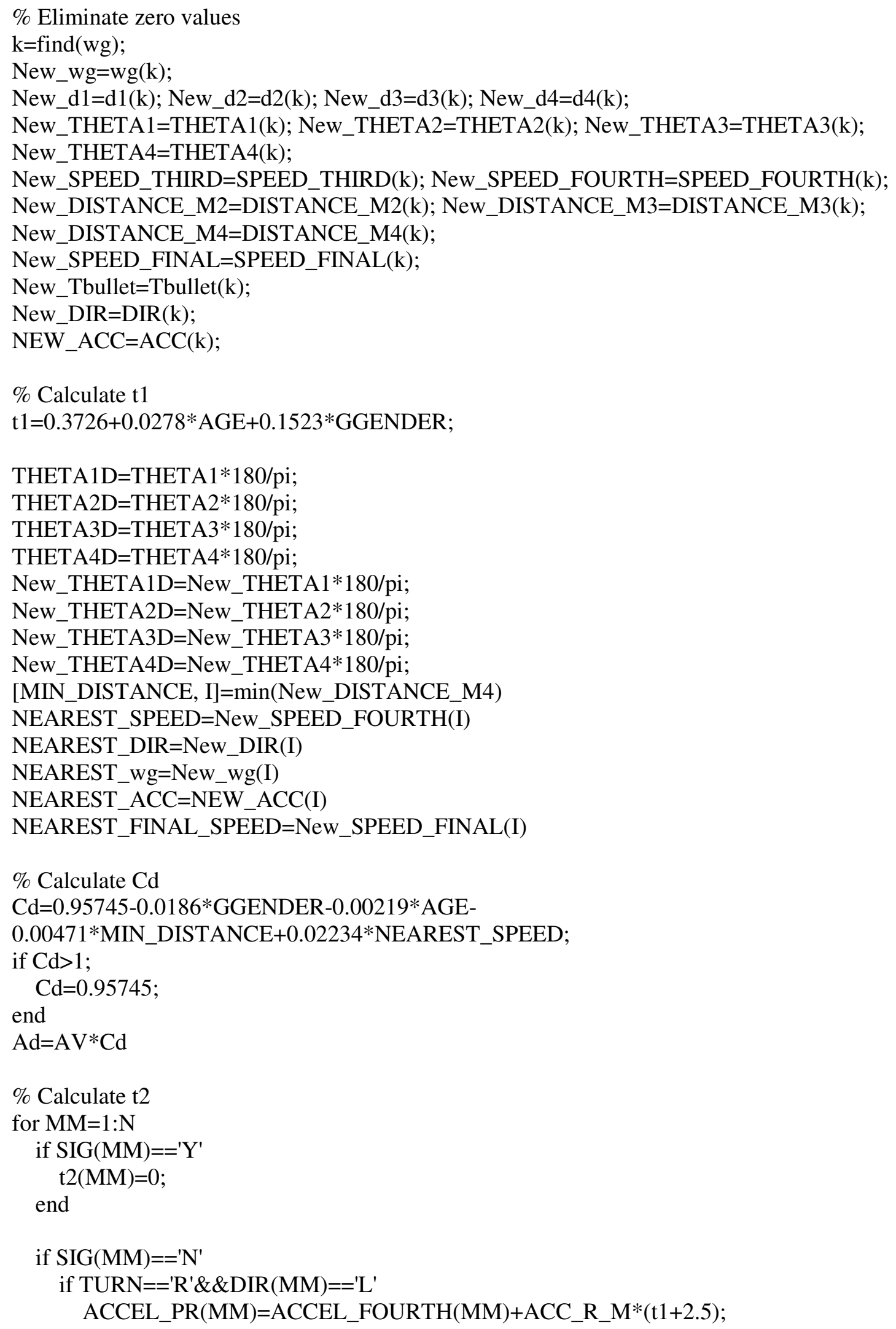


SPEED_PR(MM)=SPEED_FOURTH(MM)+(t1+2.5)*ACCEL_FOURTH(MM)+0.5*ACC_R_M*(t1 $+2.5)^{\wedge} 2$

dis_PR $(M M)=S P E E D \_F O U R T H(M M) *(t 1+2.5)+0.5 * A C C E L \_F O U R T H(M M) *(t 1+2.5)^{\wedge} 2+0.166666$ $6^{*} \mathrm{ACC} \_\mathrm{R} \_\mathrm{M}^{*}(\mathrm{t} 1+2.5)^{\wedge} 3$;

if $\mathrm{POL}=={ }^{\prime} \mathrm{L}$ '

$\mathrm{ff}=@(\mathrm{y}) \mathrm{Ad} * \mathrm{EQ} * \exp (-\mathrm{Ad} * \mathrm{y} / \mathrm{EQ})+0.7 * \mathrm{SPEED} \_\mathrm{PR}(\mathrm{MM})-\mathrm{Ad} * \mathrm{EQ}$;

$\mathrm{t} 2(\mathrm{MM})=$ fzero(ff, [0 150]);

$\mathrm{dd} 2(\mathrm{MM})=\mathrm{Ad} * \mathrm{EQ} * \mathrm{t} 2(\mathrm{MM})-\mathrm{EQ} * \mathrm{EQ} *(1-\exp (-1 * \mathrm{Ad} * \mathrm{t} 2(\mathrm{MM}) / \mathrm{EQ})) / \mathrm{Ad}-\mathrm{wg}(\mathrm{MM})$;

end

if $\mathrm{POL}=={ }^{\prime} \mathrm{C}^{\prime}$

$\mathrm{t} 2(\mathrm{MM})=0.7 * \mathrm{SPEED} \_\mathrm{PR}(\mathrm{MM}) / \mathrm{Ad}$;

$\mathrm{dd} 2(\mathrm{MM})=0.5 * \mathrm{Ad} * \mathrm{t} 2(\mathrm{MM}) * \mathrm{t} 2(\mathrm{MM})-\mathrm{wg}(\mathrm{MM})$;

end

$\operatorname{dd} 1(\mathrm{MM})=$ DISTANCE_M4(MM)-dis_PR(MM);

Tbullet $(\mathrm{MM})=0.3 *$ SPEED_PR(MM)/3.4;

dd4(MM) $=$ Tbullet $1(\mathrm{MM}) *$ SPEED_PR(MM)-1.7*Tbullet1(MM)*Tbullet1(MM);

$\operatorname{dd} 3(\mathrm{MM})=\operatorname{dd} 1(\mathrm{MM})+\mathrm{dd} 2(\mathrm{MM})-\mathrm{dd} 4(\mathrm{MM})$;

Tbullet $2(\mathrm{MM})=\mathrm{dd} 3(\mathrm{MM}) /\left(0.7 * \operatorname{SPEED} \_\mathrm{PR}(\mathrm{MM})\right)$;

Tbullet $(\mathrm{MM})=\mathrm{t} 1+2.5+$ Tbullet $1(\mathrm{MM})+\operatorname{Tbullet} 2(\mathrm{MM})$;

end

if TURN=='L'\&\&DIR(MM)=='R'

ACCEL_PR(MM)=ACCEL_FOURTH(MM)+ACC_R_M*(t1+2.5);

SPEED_PR(MM) $=$ SPEED_FOURTH(MM)+(t1+2.5)*ACCEL_FOURTH(MM $)+0.5 * A C C \_R \_M *(t 1$ $+2.5)^{\wedge} 2$

dis_PR $(\mathrm{MM})=$ SPEED_FOURTH $(\mathrm{MM}) *(\mathrm{t} 1+2.5)+0.5^{*} \mathrm{ACCEL} F$ FOURTH $(\mathrm{MM}) *(\mathrm{t} 1+2.5)^{\wedge} 2+0.166666$

6*ACC_R_M*(t1+2.5)^3;

if $\mathrm{POL}=={ }^{\prime} \mathrm{L}$ '

$\mathrm{ff}=@(\mathrm{y}) \mathrm{Ad}^{*} \mathrm{EQ} * \exp \left(-\mathrm{Ad}^{*} \mathrm{y} / \mathrm{EQ}\right)+0.7 * \operatorname{SPEED} \_P R(\mathrm{MM})-\mathrm{Ad} * \mathrm{EQ}$;

t2(MM)=fzero(ff, [0 150]);

$\mathrm{dd} 2(\mathrm{MM})=\mathrm{Ad} * \mathrm{EQ} * \mathrm{t} 2(\mathrm{MM})-\mathrm{EQ} * \mathrm{EQ}^{*}(1-\exp (-1 * \mathrm{Ad} * \mathrm{t} 2(\mathrm{MM}) / \mathrm{EQ})) / \mathrm{Ad}-\mathrm{wg}(\mathrm{MM})$;

end

if $\mathrm{POL}=={ }^{\prime} \mathrm{C}^{\prime}$

$\mathrm{t} 2(\mathrm{MM})=0.7 * \mathrm{SPEED} \_\mathrm{PR}(\mathrm{MM}) / \mathrm{Ad}$;

$\mathrm{dd} 2(\mathrm{MM})=0.5 * \mathrm{Ad} * \mathrm{t} 2(\mathrm{MM}) * \mathrm{t} 2(\mathrm{MM})-\mathrm{wg}(\mathrm{MM})$;

end

dd1(MM)=DISTANCE_M4(MM)-dis_PR(MM);

Tbullet $1(\mathrm{MM})=0.3 *$ SPEED_PR $(\mathrm{MM}) / 3.4$;

dd4(MM)=Tbullet1(MM)*SPEED_PR(MM)-1.7*Tbullet1(MM)*Tbullet1(MM);

$\mathrm{dd} 3(\mathrm{MM})=\mathrm{dd} 1(\mathrm{MM})+\mathrm{dd} 2(\mathrm{MM})-\mathrm{dd} 4(\mathrm{MM})$;

Tbullet $2(\mathrm{MM})=\mathrm{dd} 3(\mathrm{MM}) /(0.7 *$ SPEED_PR $(\mathrm{MM}))$;

Tbullet $(\mathrm{MM})=\mathrm{t} 1+2.5+$ Tbullet $1(\mathrm{MM})+$ Tbullet $2(\mathrm{MM})$;

end

if $\mathrm{TURN}=={ }^{\prime} \mathrm{L} ' \& \& \mathrm{DIR}(\mathrm{MM})=={ }^{\prime} \mathrm{L} '$

$\mathrm{S}(\mathrm{MM})=\mathrm{wg}(\mathrm{MM})+\mathrm{L}+1.065$;

if $\mathrm{POL}=={ }^{\prime} \mathrm{L}$ '

$\mathrm{f}=@(\mathrm{x}) \mathrm{x} .{ }^{\wedge} 1-\left(\mathrm{EQ} / \mathrm{Ad}^{\wedge} 2\right)+\left(\mathrm{EQ}{ }^{*} \exp \left(-\mathrm{Ad}^{*} \mathrm{x} / \mathrm{EQ}\right)\right) /\left(\mathrm{Ad}^{\wedge} 2\right)-(\mathrm{S}(\mathrm{MM}) /(\mathrm{EQ} * \mathrm{Ad})) ;$ 


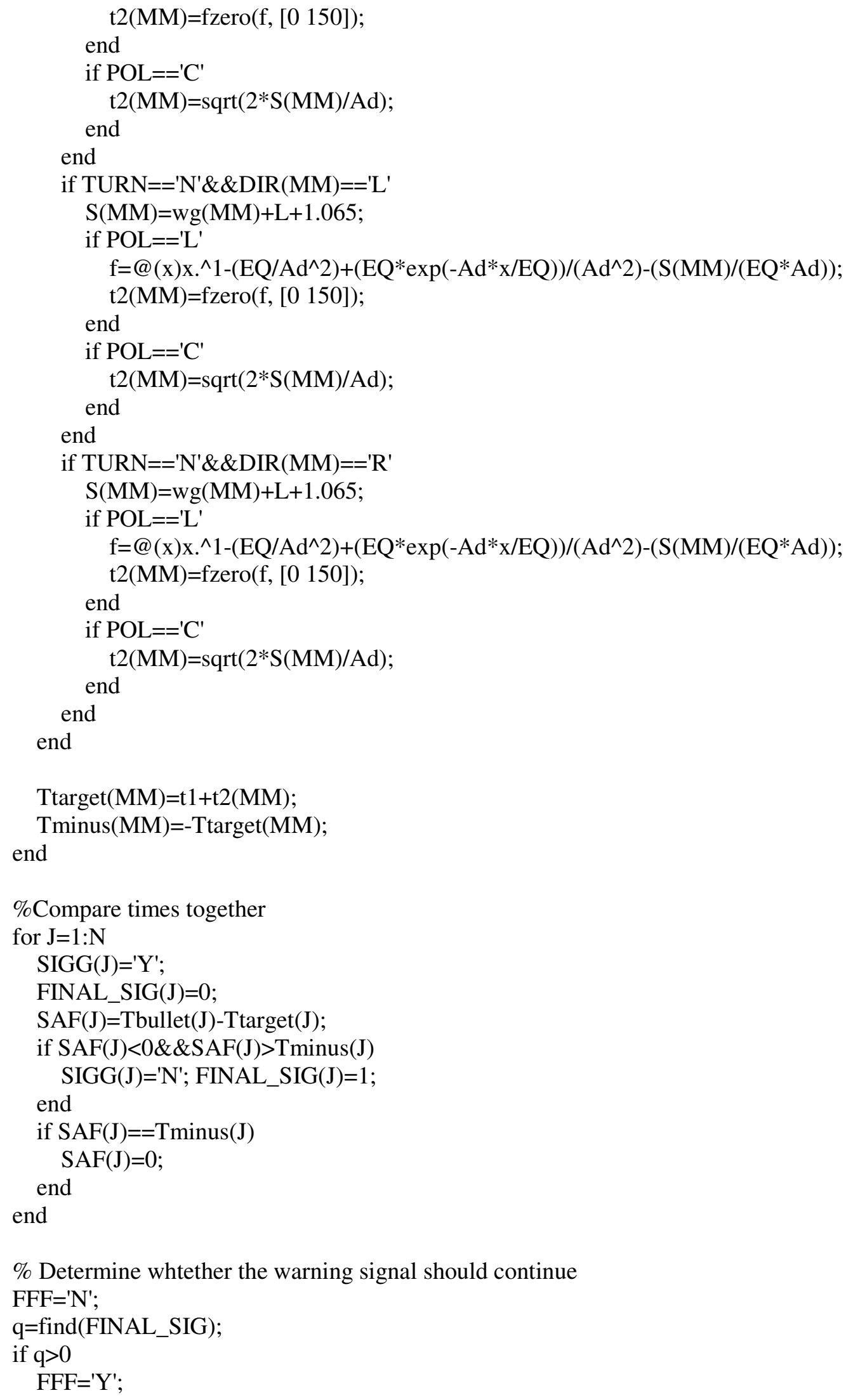




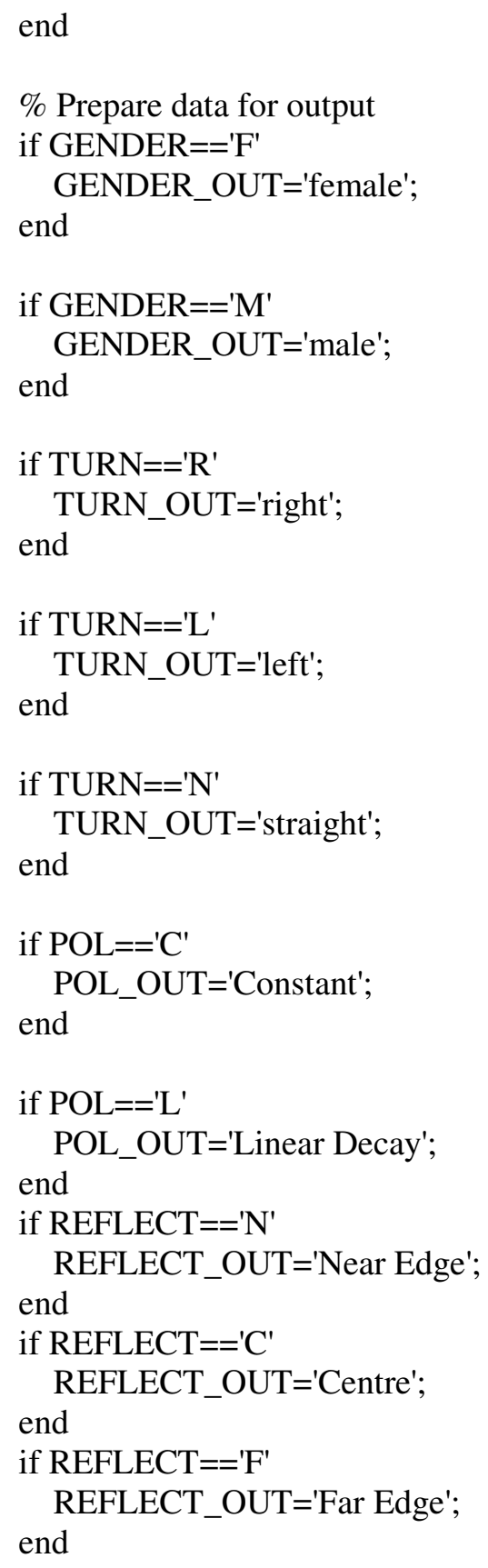


New_d1, New_d2, New_d3, New_THETA1D, New_THETA2D, New_THETA3D,

OUT_OF_COVERAGE, Cd, t1, t2, Ttarget, New_Tbullet, M

fid $=$ fopen $\left(' T W S C \_V A L I D . t x t ', ' w t '\right)$;

fprintf(fid,' $\backslash n$ ');

fprintf(fid,' $\backslash n$ ');

fprintf(fid, $* * * * * * * * * * * * * * * * * * * * * * * * * * * * * * * * * * * * * * * * * * * * * * * * * * * * * * * * * * * * * * * * * * * * * * * * * * * * * * * * * * * *$

$* * * * * * * * * * * * * * * * * * * * \ln )$;

fprintf(fid,' \n MATLAB CODE TO VALIDATE TWSC INTERSECTIONS \n $\quad$ by:

Essam Dabbour');

fprintf(fid,' ');

fprintf(fid,datestr(now));

fprintf(fid,' In \n');

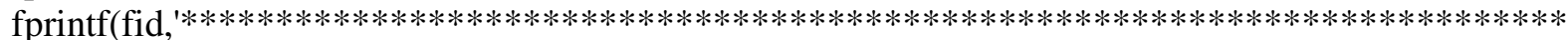

$\left.* * * * * * * * * * * * * * * * * * * * \ln \mid \mathrm{n}^{\prime}\right)$;

fprintf(fid,' $\backslash n$ ');

fprintf(fid,' 'In');

fprintf(fid,'INPUT DATA \n');

fprintf(fid,' $\left.\ln \mid n^{\prime}\right)$;

fprintf(fid,'1 - The acceleration model selected for the host vehicle is \%s \n', POL_OUT);

fprintf(fid,'2 - Maximum acceleration rate provided by the host vehicle is $\left.\% \mathrm{~g} \mathrm{~m} / \mathrm{sec} / \mathrm{sec} \backslash \mathrm{n}^{\prime}, \mathrm{AV}\right)$;

if $\mathrm{POL}=={ }^{\prime} \mathrm{L}^{\prime}$

fprintf(fid,'3 - Equilibrium (crawl) speed of the host vehicle is $\% \mathrm{~g} \mathrm{~m} / \mathrm{sec} \backslash \mathrm{n}$ ', EQ);

end

if $\mathrm{POL}=={ }^{\prime} \mathrm{C}^{\prime}$

fprintf(fid,'3 - Equilibrium (crawl) speed of the host vehicle is not applicable $\backslash n$ '); end

fprintf(fid,'4 - Length of the host vehicle is \%g m $\backslash \mathrm{n}$ ', L);

fprintf(fid,'5 - Driver is \%d years old \%s In', AGE, GENDER_OUT);

fprintf(fid,'6 - The host vehicle is heading \%s In', TURN_OUT);

fprintf(fid,'7 - Time interval for the sensor is \%g seconds $\backslash n$ ', T);

fprintf(fid,' 8 - Reflective point detected by the sensor is at \%s of the approaching vehicle $\backslash n$ ',

REFLECT_OUT);

fprintf(fid,'9 - Installation angle for the left sensor is \%g degrees \n', ALPHA_L);

fprintf(fid,'10- Installation angle for the right sensor is \%g degrees \n', ALPHA_R);

fprintf(fid,'11- Maximum azimuth angle for detection sensors is \%g degrees \n', MAX_AZIM);

fprintf(fid,'12- Number of lanes on each direction of the major road is \%d lanes $\backslash n$ ', LANES);

fprintf(fid,'13- Lane width on the major road is \%g meters $\backslash n$ ', L_WIDTH);

fprintf(fid,'14- Setback distance is \%g meters $\backslash n$ ', SETBACK);

fprintf(fid,'15- Median width is \%g meters $\backslash n$ ', MEDIAN);

fprintf(fid,'16- Skew angle of the departure road is \%g degrees $\ln \backslash n \backslash n$ ',SKEW);

fprintf(fid,'APPROACHING VEHICLES \n');

fprintf(fid,' $\ln \backslash n ')$;

fprintf(fid,'Total number of approaching vehicles from both directions is \%d vehicles, listed as follows: $\ln \backslash \mathrm{n}$ ', N);

fprintf(fid,'Veh. No. Approach. From Lane No. Distance Speed Acceleration Acc. Change

Rate $\left.\backslash n^{\prime}\right)$; 


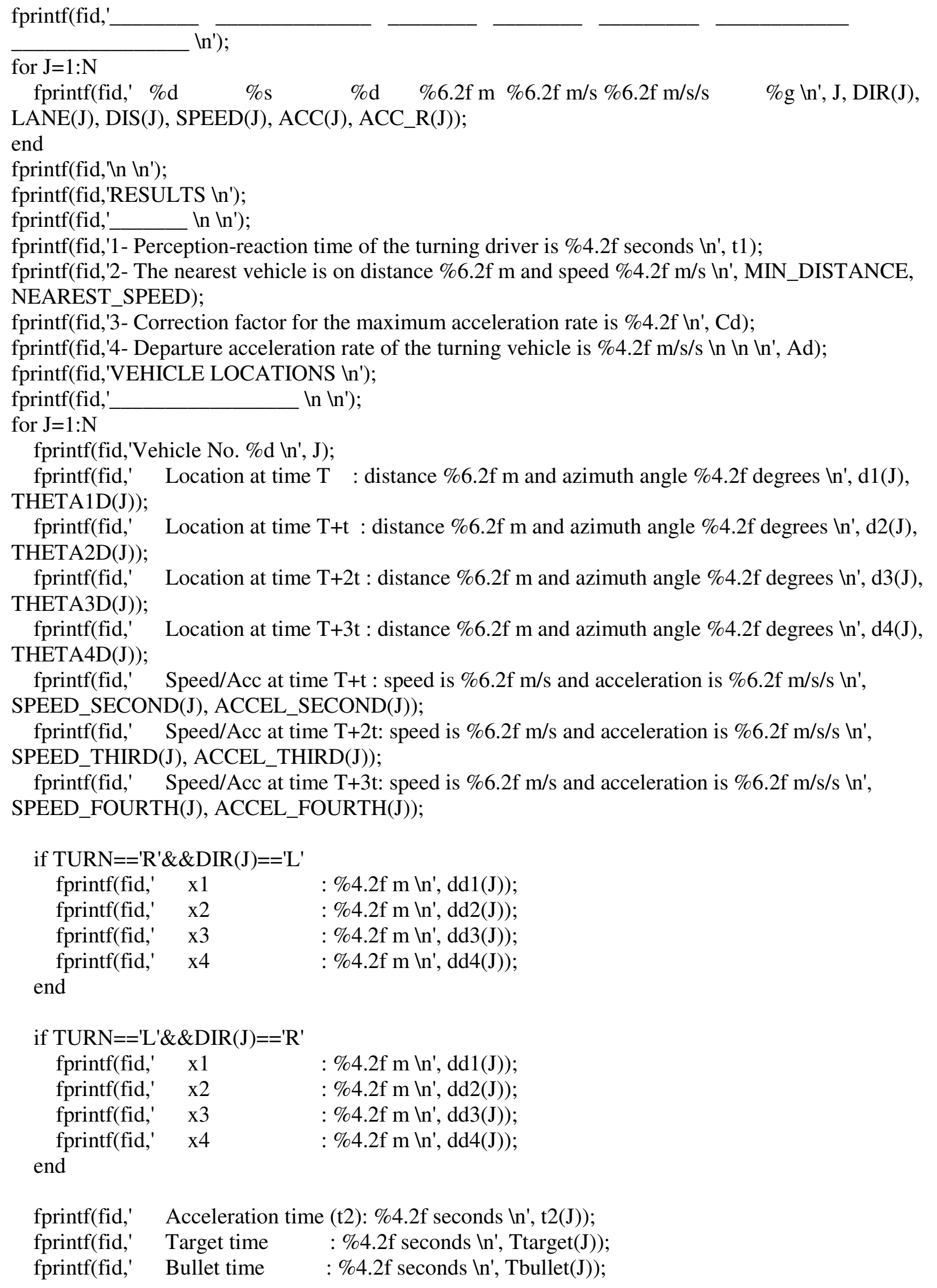




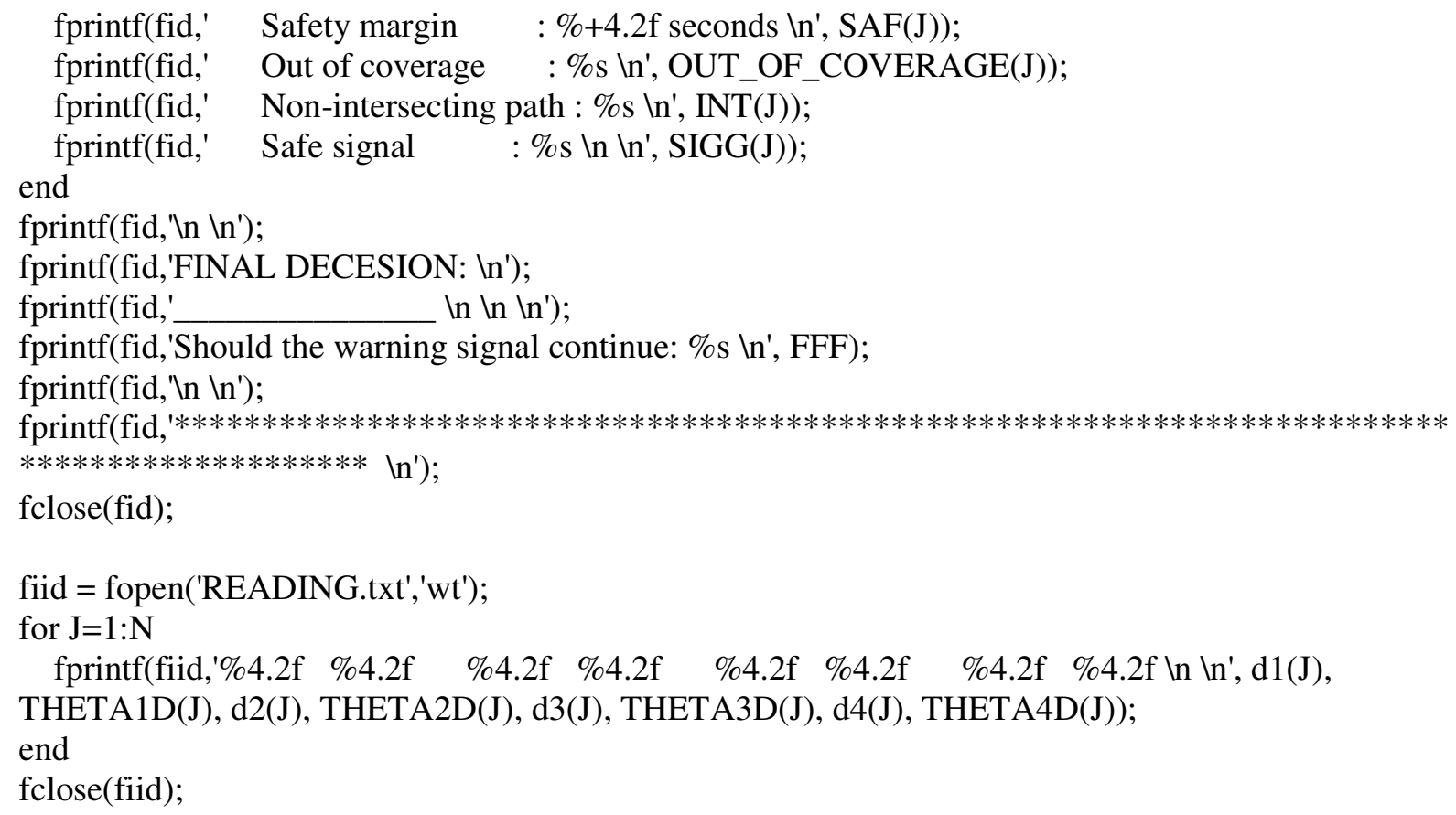




\section{Appendix " $D$ "}

\section{MATLAB Output Report for Validation Code}




\section{MATLAB CODE TO VALIDATE TWSC INTERSECTIONS}

by: Essam Dabbour $\quad$ 05-May-2009 00:58:51

*************************************************************************************************

\section{INPUT DATA}

1 - The acceleration model selected for the host vehicle is Linear Decay

2 - Maximum acceleration rate provided by the host vehicle is $3.75 \mathrm{~m} / \mathrm{sec} / \mathrm{sec}$

3 - Equilibrium (crawl) speed of the host vehicle is $40 \mathrm{~m} / \mathrm{sec}$

4 - Length of the host vehicle is $5.25 \mathrm{~m}$

5 - Driver is 28 years old male

6 - The host vehicle is heading straight

7 - Time interval for the sensor is 0.33 seconds

8 - Reflective point detected by the sensor is at Centre of the approaching vehicle

9 - Installation angle for the left sensor is 0 degrees

10- Installation angle for the right sensor is 0 degrees

11- Maximum azimuth angle for detection sensors is 90 degrees

12- Number of lanes on each direction of the major road is 3 lanes

13- Lane width on the major road is 3.5 meters

14- Setback distance is 1.75 meters

15- Median width is 2.75 meters

16- Skew angle of the departure road is 0 degrees

\section{APPROACHING VEHICLES}

Total number of approaching vehicles from both directions is 6 vehicles, listed as follows:

\begin{tabular}{|c|c|c|c|c|c|c|}
\hline Veh. No. & Approach. From & Lane No. & Distance & Speed & Acceleration & Acc. Change Rate \\
\hline 1 & $\mathrm{~L}$ & 1 & $165.00 \mathrm{~m}$ & $16.00 \mathrm{~m} / \mathrm{s}$ & $0.65 \mathrm{~m} / \mathrm{s} / \mathrm{s}$ & -0.150 \\
\hline 2 & $\mathrm{~L}$ & 2 & $175.00 \mathrm{~m}$ & $17.50 \mathrm{~m} / \mathrm{s}$ & $0.75 \mathrm{~m} / \mathrm{s} / \mathrm{s}$ & -0.100 \\
\hline 3 & $\mathrm{~L}$ & 3 & $185.00 \mathrm{~m}$ & $15.75 \mathrm{~m} / \mathrm{s}$ & $1.15 \mathrm{~m} / \mathrm{s} / \mathrm{s}$ & -0.150 \\
\hline 4 & $\mathrm{R}$ & 1 & $165.00 \mathrm{~m}$ & $15.50 \mathrm{~m} / \mathrm{s}$ & $0.25 \mathrm{~m} / \mathrm{s} / \mathrm{s}$ & -0.050 \\
\hline 5 & $\mathrm{R}$ & 2 & $215.00 \mathrm{~m}$ & $15.25 \mathrm{~m} / \mathrm{s}$ & $0.50 \mathrm{~m} / \mathrm{s} / \mathrm{s}$ & +0.050 \\
\hline 6 & $\mathrm{R}$ & 3 & $178.00 \mathrm{~m}$ & $15.25 \mathrm{~m} / \mathrm{s}$ & $0.15 \mathrm{~m} / \mathrm{s} / \mathrm{s}$ & -0.075 \\
\hline
\end{tabular}

\section{RESULTS}

1- Perception-reaction time of the turning driver is 1.15 seconds 
2- The nearest vehicle is on distance $148.87 \mathrm{~m}$ and speed $16.57 \mathrm{~m} / \mathrm{s}$

3- Correction factor for the maximum acceleration rate is 0.57

4- Departure acceleration rate of the turning vehicle is $2.12 \mathrm{~m} / \mathrm{s} / \mathrm{s}$

\section{VEHICLE LOCATIONS}

\section{Vehicle No. 1}

Location at time $\mathrm{T} \quad$ : distance $165.04 \mathrm{~m}$ and azimuth angle 88.78 degrees

Location at time $\mathrm{T}+\mathrm{t}$ : distance $159.72 \mathrm{~m}$ and azimuth angle 88.74 degrees

Location at time $\mathrm{T}+2 \mathrm{t}$ : distance $154.35 \mathrm{~m}$ and azimuth angle 88.70 degrees

Location at time $\mathrm{T}+3 \mathrm{t}$ : distance $148.91 \mathrm{~m}$ and azimuth angle 88.65 degrees

Speed/Acc at time T+t : speed is $16.21 \mathrm{~m} / \mathrm{s}$ and acceleration is $0.60 \mathrm{~m} / \mathrm{s} / \mathrm{s}$

Speed/Acc at time T+2t: speed is $16.40 \mathrm{~m} / \mathrm{s}$ and acceleration is $0.55 \mathrm{~m} / \mathrm{s} / \mathrm{s}$ Speed/Acc at time T+3t: speed is $16.57 \mathrm{~m} / \mathrm{s}$ and acceleration is $0.50 \mathrm{~m} / \mathrm{s} / \mathrm{s}$ Acceleration time (t2): 0.22 seconds

Target time $\quad: 1.37$ seconds

Bullet time $\quad: 8.84$ seconds

Safety margin $\quad:+7.47$ seconds

Out of coverage $: \mathrm{N}$

Non-intersecting path : $\mathrm{N}$

Safe signal : Y

Vehicle No. 2

Location at time $\mathrm{T} \quad$ : distance $175.14 \mathrm{~m}$ and azimuth angle 87.71 degrees

Location at time $\mathrm{T}+\mathrm{t}$ : distance $169.33 \mathrm{~m}$ and azimuth angle 87.63 degrees

Location at time $\mathrm{T}+2 \mathrm{t}$ : distance $163.44 \mathrm{~m}$ and azimuth angle 87.55 degrees

Location at time $\mathrm{T}+3 \mathrm{t}$ : distance $157.48 \mathrm{~m}$ and azimuth angle 87.45 degrees Speed/Acc at time T+t : speed is $17.74 \mathrm{~m} / \mathrm{s}$ and acceleration is $0.72 \mathrm{~m} / \mathrm{s} / \mathrm{s}$ Speed/Acc at time T+2t: speed is $17.97 \mathrm{~m} / \mathrm{s}$ and acceleration is $0.68 \mathrm{~m} / \mathrm{s} / \mathrm{s}$ Speed/Acc at time T+3t: speed is $18.19 \mathrm{~m} / \mathrm{s}$ and acceleration is $0.65 \mathrm{~m} / \mathrm{s} / \mathrm{s}$ Acceleration time (t2): 0.30 seconds

Target time $\quad: 1.45$ seconds

Bullet time : 7.97 seconds

Safety margin $\quad:+6.53$ seconds

Out of coverage $: \mathrm{N}$

Non-intersecting path : N

Safe signal $\quad: Y$

Vehicle No. 3

Location at time $\mathrm{T} \quad$ : distance $185.30 \mathrm{~m}$ and azimuth angle 86.75 degrees Location at time $\mathrm{T}+\mathrm{t}$ : distance $180.05 \mathrm{~m}$ and azimuth angle 86.66 degrees Location at time $\mathrm{T}+2 \mathrm{t}$ : distance $174.68 \mathrm{~m}$ and azimuth angle 86.55 degrees Location at time $\mathrm{T}+3 \mathrm{t}$ : distance $169.19 \mathrm{~m}$ and azimuth angle 86.44 degrees Speed/Acc at time $\mathrm{T}+\mathrm{t}$ : speed is $16.12 \mathrm{~m} / \mathrm{s}$ and acceleration is $1.10 \mathrm{~m} / \mathrm{s} / \mathrm{s}$ Speed/Acc at time T+2t: speed is $16.48 \mathrm{~m} / \mathrm{s}$ and acceleration is $1.05 \mathrm{~m} / \mathrm{s} / \mathrm{s}$ Speed/Acc at time T+3t: speed is $16.81 \mathrm{~m} / \mathrm{s}$ and acceleration is $1.00 \mathrm{~m} / \mathrm{s} / \mathrm{s}$ Acceleration time (t2): 0.37 seconds

Target time $\quad: 1.52$ seconds 
Bullet time $\quad: 8.76$ seconds

Safety margin $\quad:+7.23$ seconds

Out of coverage : N

Non-intersecting path : $\mathrm{N}$

Safe signal : $\mathrm{Y}$

Vehicle No. 4

Location at time $\mathrm{T} \quad$ : distance $165.85 \mathrm{~m}$ and azimuth angle 84.20 degrees

Location at time $\mathrm{T}+\mathrm{t}$ : distance $160.75 \mathrm{~m}$ and azimuth angle 84.02 degrees

Location at time $\mathrm{T}+2 \mathrm{t}$ : distance $155.62 \mathrm{~m}$ and azimuth angle 83.82 degrees

Location at time $\mathrm{T}+3 \mathrm{t}$ : distance $150.48 \mathrm{~m}$ and azimuth angle 83.61 degrees

Speed/Acc at time T+t : speed is $15.58 \mathrm{~m} / \mathrm{s}$ and acceleration is $0.23 \mathrm{~m} / \mathrm{s} / \mathrm{s}$

Speed/Acc at time T+2t: speed is $15.65 \mathrm{~m} / \mathrm{s}$ and acceleration is $0.22 \mathrm{~m} / \mathrm{s} / \mathrm{s}$

Speed/Acc at time T+3t: speed is $15.72 \mathrm{~m} / \mathrm{s}$ and acceleration is $0.20 \mathrm{~m} / \mathrm{s} / \mathrm{s}$

Acceleration time (t2): 0.51 seconds

Target time $\quad: 1.66$ seconds

Bullet time $\quad: 9.39$ seconds

Safety margin $\quad:+7.73$ seconds

Out of coverage : $\mathrm{N}$

Non-intersecting path : $\mathrm{N}$

Safe signal : Y

Vehicle No. 5

Location at time $\mathrm{T}$ : distance $215.95 \mathrm{~m}$ and azimuth angle 84.62 degrees

Location at time $\mathrm{T}+\mathrm{t}$ : distance $210.91 \mathrm{~m}$ and azimuth angle 84.49 degrees

Location at time $\mathrm{T}+2 \mathrm{t}$ : distance $205.82 \mathrm{~m}$ and azimuth angle 84.35 degrees

Location at time $\mathrm{T}+3 \mathrm{t}$ : distance $200.67 \mathrm{~m}$ and azimuth angle 84.21 degrees Speed/Acc at time T+t : speed is $15.42 \mathrm{~m} / \mathrm{s}$ and acceleration is $0.52 \mathrm{~m} / \mathrm{s} / \mathrm{s}$ Speed/Acc at time T+2t: speed is $15.59 \mathrm{~m} / \mathrm{s}$ and acceleration is $0.53 \mathrm{~m} / \mathrm{s} / \mathrm{s}$ Speed/Acc at time T+3t: speed is $15.77 \mathrm{~m} / \mathrm{s}$ and acceleration is $0.55 \mathrm{~m} / \mathrm{s} / \mathrm{s}$ Acceleration time (t2): 0.59 seconds

Target time $\quad: 1.74$ seconds

Bullet time $\quad: 10.26$ seconds

Safety margin $\quad:+8.52$ seconds

Out of coverage : $\mathrm{N}$

Non-intersecting path : N

Safe signal $\quad: Y$

Vehicle No. 6

Location at time $\mathrm{T} \quad$ : distance $179.58 \mathrm{~m}$ and azimuth angle 82.40 degrees

Location at time $\mathrm{T}+\mathrm{t}$ : distance $174.58 \mathrm{~m}$ and azimuth angle 82.18 degrees

Location at time $\mathrm{T}+2 \mathrm{t}$ : distance $169.58 \mathrm{~m}$ and azimuth angle 81.95 degrees

Location at time $\mathrm{T}+3 \mathrm{t}$ : distance $164.56 \mathrm{~m}$ and azimuth angle 81.70 degrees

Speed/Acc at time $\mathrm{T}+\mathrm{t}:$ speed is $15.30 \mathrm{~m} / \mathrm{s}$ and acceleration is $0.13 \mathrm{~m} / \mathrm{s} / \mathrm{s}$

Speed/Acc at time T+2t: speed is $15.33 \mathrm{~m} / \mathrm{s}$ and acceleration is $0.10 \mathrm{~m} / \mathrm{s} / \mathrm{s}$

Speed/Acc at time T+3t: speed is $15.36 \mathrm{~m} / \mathrm{s}$ and acceleration is $0.08 \mathrm{~m} / \mathrm{s} / \mathrm{s}$

Acceleration time (t2): 0.66 seconds

Target time $\quad: 1.81$ seconds

Bullet time $\quad: 11.52$ seconds

Safety margin $\quad:+9.70$ seconds 
Out of coverage $: \mathrm{N}$

Non-intersecting path : $\mathrm{N}$

Safe signal : $: Y$

FINAL DECESION:

Should the warning signal continue: $\mathrm{N}$

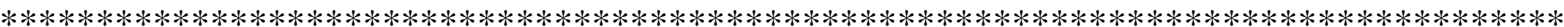




\section{Appendix " $E$ "}

MATLAB Code to Simulate Detection Sensor 
$\%$ MATLAB code to validate TWSC intersections by simulating detection sensors $\%$ by: Essam Dabbour

clc

clear all

\%Input vehicle data

POL=input('Input the model of acceleration used for host vehicle (Constant / Linear decay) [C]: ','s'); if isempty(POL)

$\mathrm{POL}=$ 'C';

end

if $\mathrm{POL}==^{\prime} \mathrm{C}^{\prime}$

$\mathrm{AV}=$ input('Input the maximum acceleration provided by the host vehicle in $\mathrm{m} / \mathrm{sec} / \mathrm{sec}$ [2.75]: ');

if isempty(AV)

$\mathrm{AV}=2.75$

end

end

if $\mathrm{POL}==^{\prime} \mathrm{L} '$

$\mathrm{AV}=$ input('Input the maximum initial acceleration provided by the host vehicle in $\mathrm{m} / \mathrm{sec} / \mathrm{sec}$ [3.75]: ');

if isempty $(\mathrm{AV})$

$\mathrm{AV}=3.75$

end

$\mathrm{EQ}=$ input('Input the equilibrium (crawl) speed of the host vehicle in $\mathrm{m} / \mathrm{sec}$ [40]: ');

if isempty(EQ)

$\mathrm{EQ}=40$;

end

end

$\mathrm{L}=$ input('Input the length of the host vehicle in meters [5.25 m]: ');

if isempty(L)

$\mathrm{L}=5.25$;

end

\%Input driver data

AGE=input('Input the age of the driver in years [28]: ');

if isempty(AGE)

$$
\mathrm{AGE}=28
$$

end

GENDER=input('Input the gender of the driver M/F [M]: ','s');

if isempty(GENDER)

GENDER = 'M';

end

if GENDER $==$ 'M'

GGENDER $=0$;

end

if GENDER $==$ ' $\mathrm{F}$ '

GGENDER=1;

end

TURN=input('Input driver turning signal L/R or "N" for no signal [N]: ','s');

if isempty(TURN) 


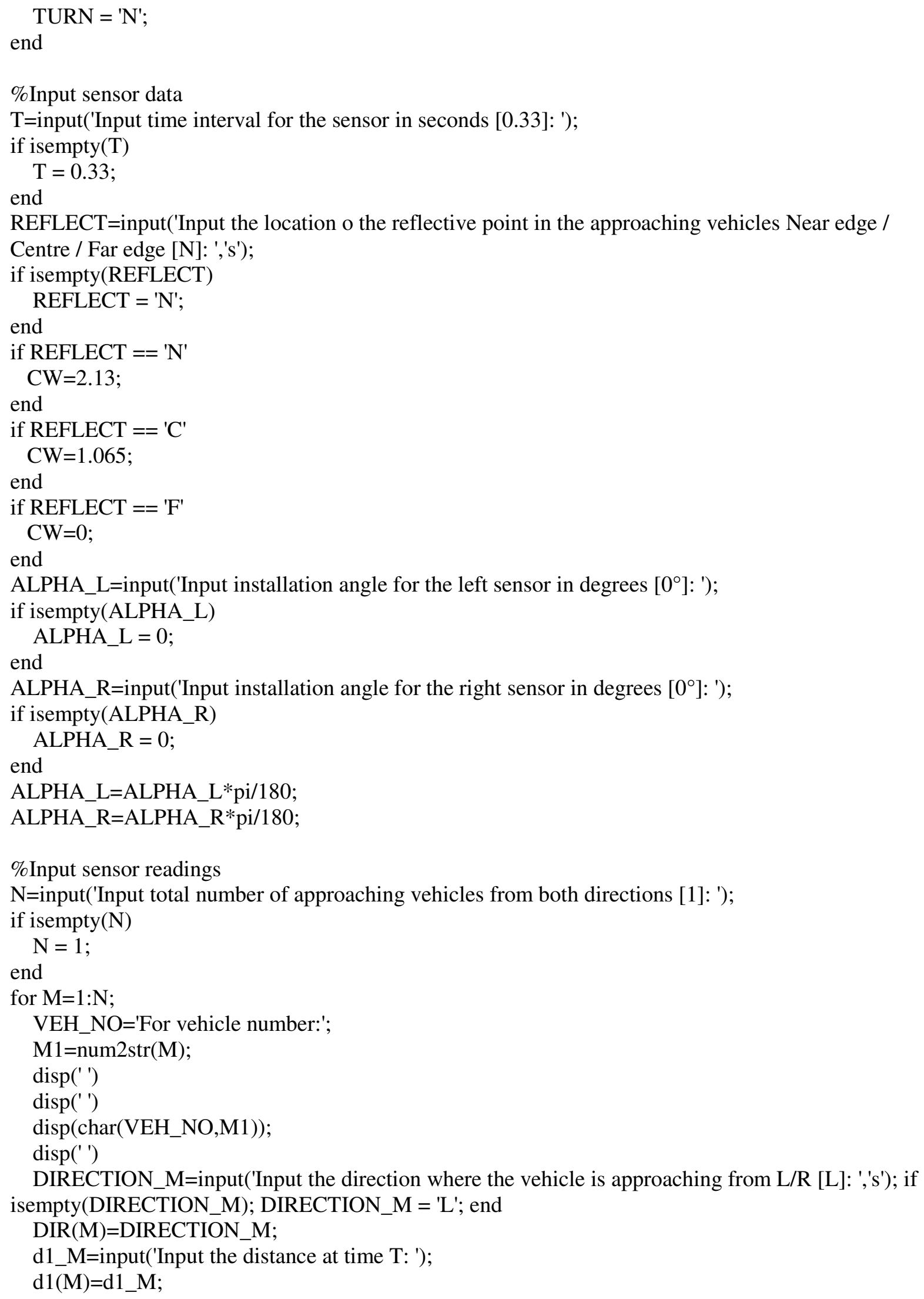


THETA1_M=input('Input azimuth angle at time T: ');

THETA1_M=THETA1_M*pi/180;

THETA1(M)=THETA1_M;

d2_M=input('Input the distance at time $\mathrm{T}+\mathrm{t}$ : ');

$\mathrm{d} 2(\mathrm{M})=\mathrm{d} 2 \_\mathrm{M}$;

THETA2_M=input('Input azimuth angle at time $\mathrm{T}+\mathrm{t}$ : ');

THETA2_M=THETA2_M*pi/180;

THETA2 $(\mathrm{M})=$ THETA2_M;

d3_M=input('Input the distance at time $\mathrm{T}+2 \mathrm{t}$ : ');

$\mathrm{d} 3(\mathrm{M})=\mathrm{d} 3$ _M;

THETA3_M=input('Input azimuth angle at time $\mathrm{T}+2 \mathrm{t}$ : ');

THETA3_M=THETA3_M*pi/180;

THETA3(M)=THETA3_M;

d4_M=input('Input the distance at time T+3t: ');

$\mathrm{d} 4(\mathrm{M})=\mathrm{d} 4$ _M;

THETA4_M=input('Input azimuth angle at time T+3t: ');

THETA4_M=THETA4_M*pi/180;

THETA4(M)=THETA4_M;

$\%$ Safe signal if the equipped vehicle is turning right and the

$\%$ approaching vehicle is approaching from right

$\operatorname{SIG}(\mathrm{M})=$ 'N'; INT(M)='N';

if TURN=='R'\&\& DIRECTION_M=='R'

Tbullet(M)=0; SIG(M)='Y'; INT(M)='Y';

end

$\%$ Calculate speed and acceleration

$\mathrm{dv} 1(\mathrm{M})=\operatorname{sqrt}((\mathrm{d} 1(\mathrm{M}) * \mathrm{~d} 1(\mathrm{M}))+(\mathrm{d} 2(\mathrm{M}) * \mathrm{~d} 2(\mathrm{M}))-2 * \mathrm{~d} 1(\mathrm{M}) * \mathrm{~d} 2(\mathrm{M}) * \cos (\operatorname{THETA} 1(\mathrm{M})-\operatorname{THETA} 2(\mathrm{M})))$;

$\mathrm{dv} 2(\mathrm{M})=\operatorname{sqrt}((\mathrm{d} 2(\mathrm{M}) * \mathrm{~d} 2(\mathrm{M}))+(\mathrm{d} 3(\mathrm{M}) * \mathrm{~d} 3(\mathrm{M}))-2 * \mathrm{~d} 2(\mathrm{M}) * \mathrm{~d} 3(\mathrm{M}) * \cos ($ THETA2(M)-THETA3(M)) ;

$\mathrm{dv} 3(\mathrm{M})=\operatorname{sqrt}((\mathrm{d} 3(\mathrm{M}) * \mathrm{~d} 3(\mathrm{M}))+(\mathrm{d} 4(\mathrm{M}) * \mathrm{~d} 4(\mathrm{M}))-2 * \mathrm{~d} 3(\mathrm{M}) * \mathrm{~d} 4(\mathrm{M}) * \cos ($ THETA3(M)-THETA4(M)));

$\mathrm{r}(\mathrm{M})=(\mathrm{dv} 1(\mathrm{M})-2 * \operatorname{dv} 2(\mathrm{M})+\mathrm{dv} 3(\mathrm{M})) /(\mathrm{T} * \mathrm{~T} * \mathrm{~T})$

$\mathrm{a} 0(\mathrm{M})=(3 * \mathrm{dv} 2(\mathrm{M})-2 * \mathrm{dv} 1(\mathrm{M})-\mathrm{dv} 3(\mathrm{M})) /(\mathrm{T} * \mathrm{~T})$;

$\mathrm{v} 0(\mathrm{M})=(1.83333 * \mathrm{dv} 1(\mathrm{M})-1.1666 * \mathrm{dv} 2(\mathrm{M})+0.3333 * \mathrm{dv} 3(\mathrm{M})) / \mathrm{T}$;

$\mathrm{a} 3(\mathrm{M})=\mathrm{a} 0(\mathrm{M})+3 * \mathrm{r}(\mathrm{M}) * \mathrm{~T}$;

$\mathrm{v} 3(\mathrm{M})=\mathrm{v} 0(\mathrm{M})+3 * \mathrm{~T} * \mathrm{a} 0(\mathrm{M})+4.5 * \mathrm{~T} * \mathrm{~T} * \mathrm{r}(\mathrm{M})$;

$\mathrm{wf} 1(\mathrm{M})=\mathrm{d} 1(\mathrm{M}) * \mathrm{~d} 2(\mathrm{M}) * \sin ($ THETA1(M)-THETA2(M))/dv1(M);

$\mathrm{wf} 2(\mathrm{M})=\mathrm{d} 2(\mathrm{M}) * \mathrm{~d} 3(\mathrm{M}) * \sin ($ THETA2(M)-THETA3(M))/dv2(M);

$\mathrm{wf} 3(\mathrm{M})=\mathrm{d} 3(\mathrm{M}) * \mathrm{~d} 4(\mathrm{M}) * \sin (\mathrm{THETA} 3(\mathrm{M})-\mathrm{THETA} 4(\mathrm{M})) / \mathrm{dv} 3(\mathrm{M})$;

$\mathrm{wf}(\mathrm{M})=(\mathrm{wf} 1(\mathrm{M})+\mathrm{wf} 2(\mathrm{M})+\mathrm{wf} 3(\mathrm{M})) / 3$;

$\mathrm{df}(\mathrm{M})=\operatorname{sqrt}(\mathrm{d} 4(\mathrm{M}) * \mathrm{~d} 4(\mathrm{M})-\mathrm{wf}(\mathrm{M}) * \mathrm{wf}(\mathrm{M}))$;

\%Calculate Tbullet

Tbullet $(\mathrm{M})=0$;

if $\operatorname{SIG}(\mathrm{M})=={ }^{\prime} \mathrm{N}^{\prime}$

$\mathrm{p}=[0.1666 * \mathrm{r}(\mathrm{M}) 0.5 * \mathrm{a} 3(\mathrm{M}) \mathrm{v} 3(\mathrm{M})-\mathrm{df}(\mathrm{M})]$;

$\mathrm{y}=\operatorname{roots}(\mathrm{p})$;

for $\mathrm{hh}=1$ :numel $(\mathrm{y})$

if $\operatorname{imag}(\mathrm{y}(\mathrm{hh}))==0$

Tbullet(M) $=\mathrm{y}(\mathrm{hh})$;

end 


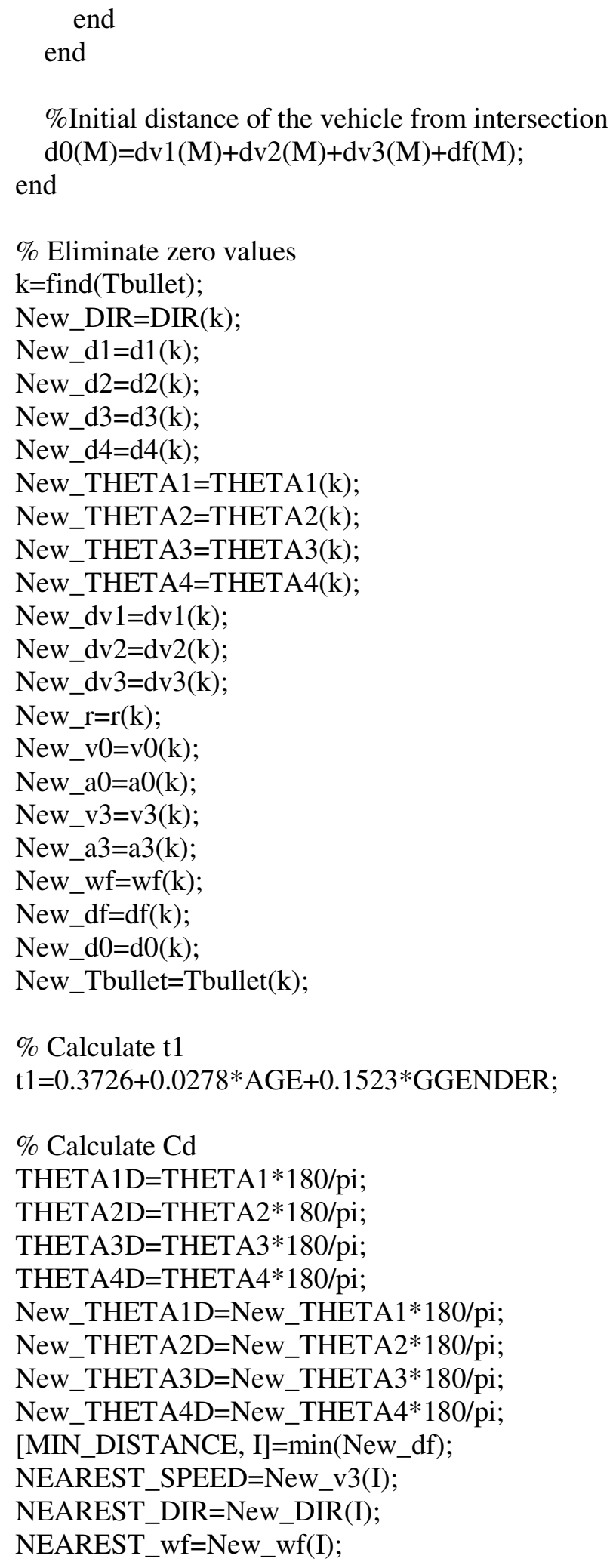




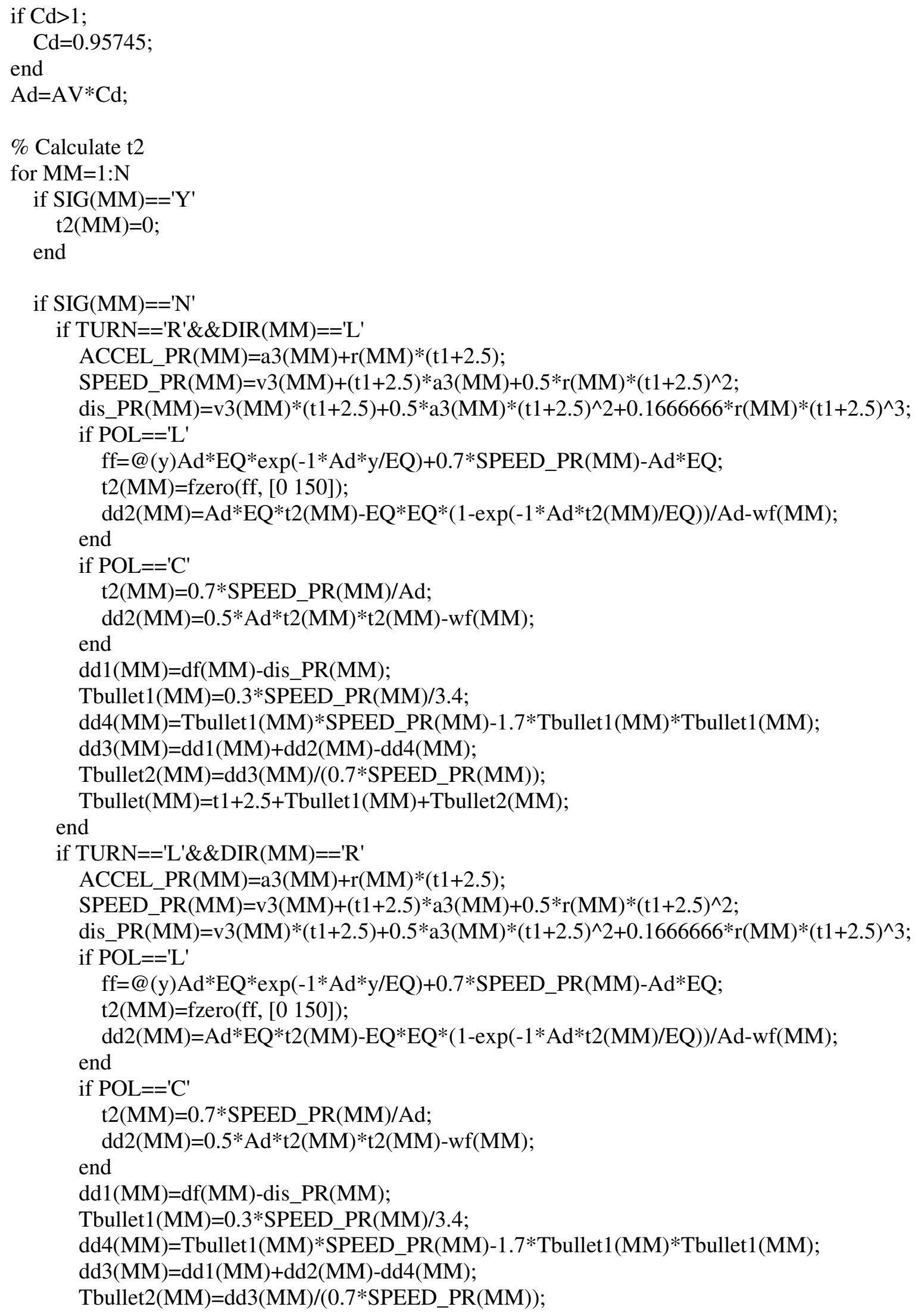




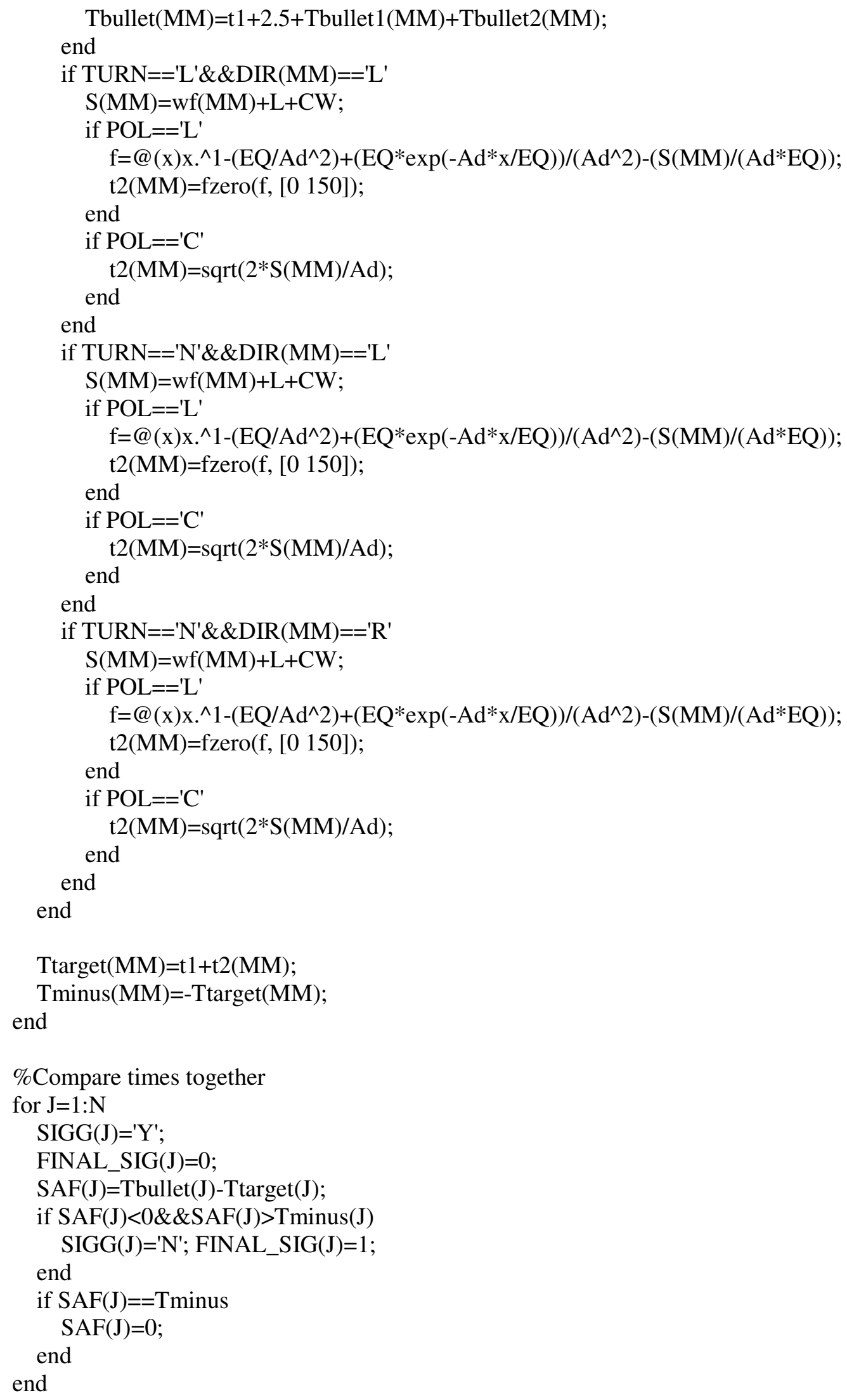




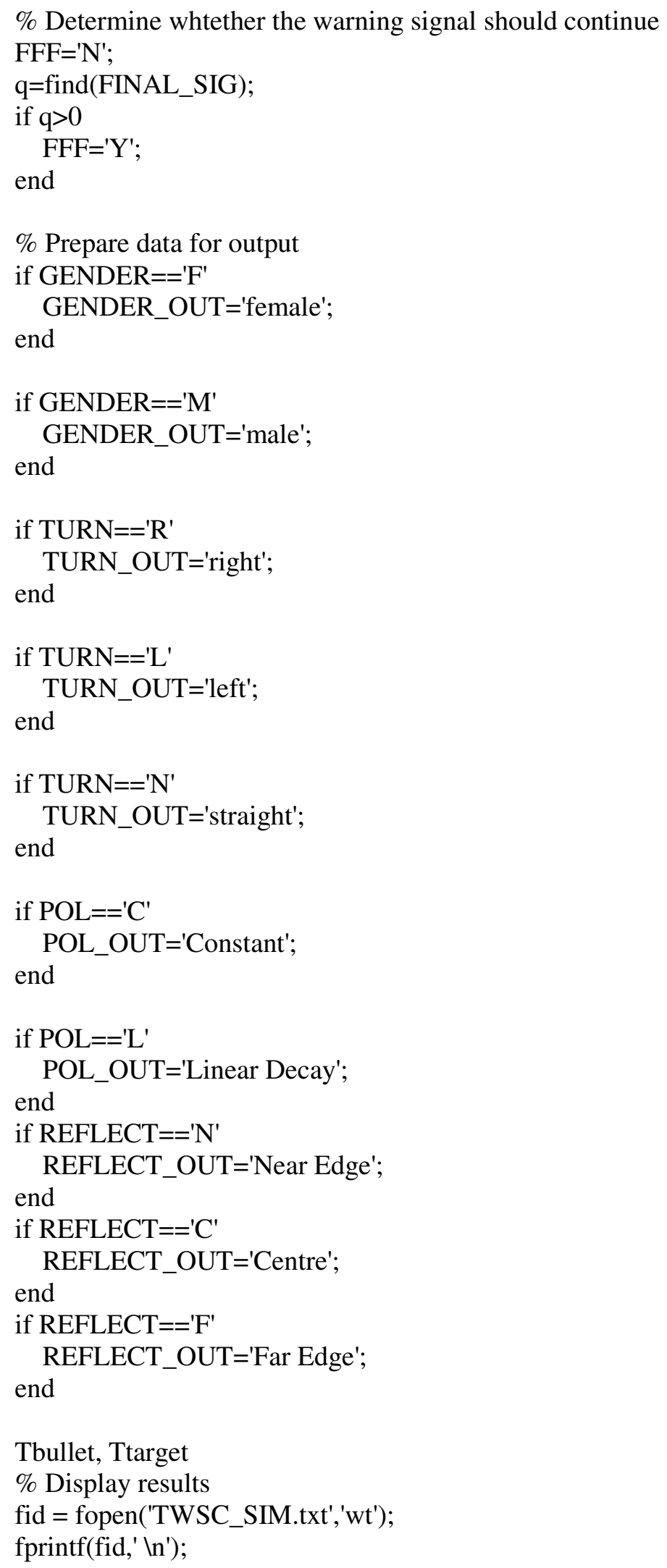


fprintf(fid,' 'ln');

fprintf(fid, $* * * * * * * * * * * * * * * * * * * * * * * * * * * * * * * * * * * * * * * * * * * * * * * * * * * * * * * * * * * * * * * * * * * * * * * * * * * * *$

$\left.* * * * * * * * * * * * * * * * * * * * \quad \mathrm{n}^{\prime}\right)$;

fprintf(fid,' In MATLAB CODE TO VALIDATE TWSC INTERSECTIONS BY

SIMULATING DETECTORS $\backslash \mathrm{n} \quad$ by: Essam Dabbour');

fprintf(fid,' ' ');

fprintf(fid,datestr(now));

fprintf(fid,' $\backslash \mathrm{n} \backslash \mathrm{n}$ ');

fprintf(fid, $* * * * * * * * * * * * * * * * * * * * * * * * * * * * * * * * * * * * * * * * * * * * * * * * * * * * * * * * * * * * * * * * * * * * * * * * * * * * *$

$\left.* * * * * * * * * * * * * * * * * * * * \ln \backslash \mathrm{n}^{\prime}\right)$;

fprintf(fid,' $\backslash n$ ');

fprintf(fid,' 'ln');

fprintf(fid,'INPUT DATA $\backslash n$ ');

fprintf(fid,'_ In $\backslash n$ ');

fprintf(fid,'1 - The acceleration model selected for the host vehicle is \%s $\backslash n$ ', POL_OUT);

fprintf(fid,'2 - Maximum acceleration rate provided by the host vehicle is $\% \mathrm{~g} \mathrm{~m} / \mathrm{sec} / \mathrm{sec} \backslash \mathrm{n}$ ', AV);

if $\mathrm{POL}=={ }^{\prime} \mathrm{L}$

fprintf(fid,'3 - Equilibrium (crawl) speed of the host vehicle is $\% \mathrm{~g} \mathrm{~m} / \mathrm{sec} \backslash \mathrm{n}$ ', EQ);

end

if $\mathrm{POL}=={ }^{\prime} \mathrm{C}^{\prime}$

fprintf(fid,'3 - Equilibrium (crawl) speed of the host vehicle is not applicable $\backslash n '$ ');

end

fprintf(fid,'4 - Length of the host vehicle is \%g m $\backslash \mathrm{n}$ ', L);

fprintf(fid,'5 - Driver is \%d years old \%s In', AGE, GENDER_OUT);

fprintf(fid,'6 - The host vehicle is heading \%s In', TURN_OUT);

fprintf(fid,'7 - Time interval for the sensor is \%g seconds $\backslash n$ ', T);

fprintf(fid,'8 - Reflective point detected by the sensor is at \%s of the approaching vehicle $\backslash n$ ',

REFLECT_OUT);

fprintf(fid,'9 - Installation angle for the left sensor is \%g degrees \n', ALPHA_L);

fprintf(fid,'10- Installation angle for the right sensor is \%g degrees $\ln \backslash n \backslash n '$ ', ALPHA_R);

fprintf(fid,'VEHICLE LOCATIONS AND RESULTS \n');

fprintf(fid,' $\ln ')$;

for $\mathrm{J}=1: \mathrm{N}$

fprintf(fid,'Vehicle No. \%d \n', J);

fprintf(fid,' Location at time $\mathrm{T}$ : distance $\% 6.2 \mathrm{f} \mathrm{m}$ and azimuth angle \%4.2f degrees $\backslash \mathrm{n}$ ', $\mathrm{d} 1(\mathrm{~J})$,

THETA1D $(J))$;

fprintf(fid,' Location at time $\mathrm{T}+\mathrm{t}$ : distance \%6.2f $\mathrm{m}$ and azimuth angle \%4.2f degrees $\backslash \mathrm{n}$ ', $\mathrm{d} 2(\mathrm{~J})$,

THETA2D (J));

fprintf(fid,' Location at time T+2t: distance \%6.2f $\mathrm{m}$ and azimuth angle \%4.2f degrees $\backslash \mathrm{n}$ ', $\mathrm{d} 3(\mathrm{~J})$,

$\operatorname{THETA3D}(\mathrm{J}))$;

fprintf(fid,' Location at time T+3t: distance \%6.2f $\mathrm{m}$ and azimuth angle \%4.2f degrees $\backslash \mathrm{n}$ ', $\mathrm{d} 4(\mathrm{~J})$,

THETA4D(J));

fprintf(fid,' Approaching from :\%s $\backslash \mathrm{n}$ ', DIR(J));

fprintf(fid,' Initial distance : \%4.2f $\left.\backslash \mathrm{n}^{\prime}, \mathrm{d} 0(\mathrm{~J})\right)$;

fprintf(fid,' Offset distance :\%4.2f m $\backslash \mathrm{n}$ ', wf(J));

fprintf(fid,' Initial speed $\quad: \% 4.2 \mathrm{f} \mathrm{m} / \mathrm{sec} \backslash \mathrm{n}$ ', $\mathrm{v} 0(\mathrm{~J}))$;

fprintf(fid,' Initial Acceleration : \%6.2f m/sec/sec $\backslash \mathrm{n}$ ', a0(J)); 
fprintf(fid,' Accel. rate of change: $\left.\% 6.2 \mathrm{f} \mathrm{m} / \mathrm{sec} / \mathrm{sec} / \mathrm{sec} \backslash \mathrm{n}^{\prime}, \mathrm{r}(\mathrm{J})\right)$;

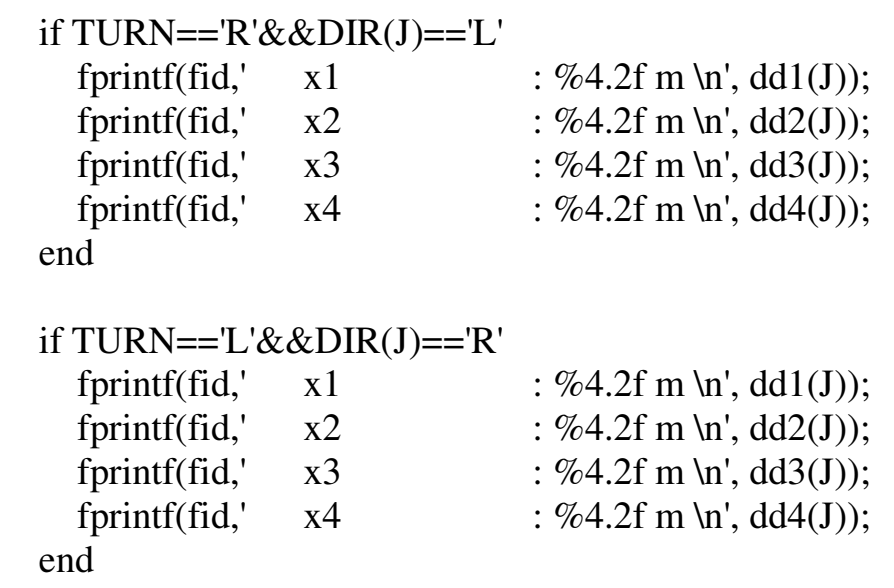

fprintf(fid,' Acceleration time (t2): \%4.2f seconds $\backslash \mathrm{n}$ ', t2(J));

fprintf(fid,' $\quad$ Target time $\% 4.2 \mathrm{f}$ seconds $\backslash \mathrm{n}$ ', Ttarget(J));

fprintf(fid,' $\quad$ Bullet time $\% 4.2 \mathrm{f}$ seconds $\backslash \mathrm{n}$ ', Tbullet(J));

fprintf(fid,' $\quad$ Safety margin $\quad: \%+4.2 \mathrm{f}$ seconds $\backslash \mathrm{n}$ ', $\operatorname{SAF}(\mathrm{J}))$;

fprintf(fid,' Non-intersecting path: \%s $\backslash n$ ', INT(J));

fprintf(fid,' $\quad$ Safe signal $\%$ s $\backslash n \backslash n$ ', $\operatorname{SIGG}(J))$;

end

fprintf(fid,' 'In $\backslash n ')$;

fprintf(fid,'Results \n');

fprintf(fid,'_ ln $\backslash n$ ');

fprintf(fid,' 1- Perception-reaction time of the turning driver is $\% 4.2 \mathrm{f}$ seconds $\backslash \mathrm{n}$ ', $\mathrm{t} 1$ );

fprintf(fid,' 2- The nearest vehicle is on distance $\% 6.2 \mathrm{f} \mathrm{m}$ and speed $\% 4.2 \mathrm{f} \mathrm{m} / \mathrm{s} \backslash \mathrm{n}$,

MIN_DISTANCE, NEAREST_SPEED);

fprintf(fid,' 3- Correction factor for the maximum acceleration rate is $\% 4.2 \mathrm{f} \backslash \mathrm{n}$ ', Cd);

fprintf(fid,' 4- Departure acceleration rate of the turning vehicle is $\% 4.2 \mathrm{f} \mathrm{m} / \mathrm{s} / \mathrm{s} \backslash \mathrm{n} \backslash \mathrm{n} \backslash \mathrm{n}$ ', Ad);

fprintf(fid,'FINAL DECESION: \n');

fprintf(fid,' $\ln \ln \backslash n$ ');

fprintf(fid,'Should the warning signal continue: \%s \n', FFF);

fprintf(fid,' '\n \n');

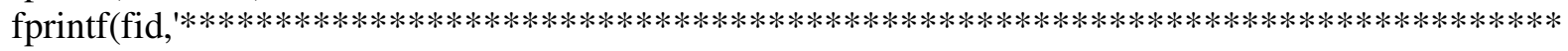

$\left.* * * * * * * * * * * * * * * * * * * * \ln )^{\prime}\right)$

fclose(fid); 


\section{Appendix "F"}

MATLAB Output Report for Sensor Simulation Code 


\title{
MATLAB CODE TO VALIDATE TWSC INTERSECTIONS BY SIMULATING DETECTORS
}

\author{
by: Essam Dabbour \\ 05-May-2009 01:11:37
}

$* * * * * * * * * * * * * * * * * * * * * * * * * * * * * * * * * * * * * * * * * * * * * * * * * * * * * * * * * * * * * * * * * * * * * * * * * * * * * * * * * * * * * * * * *$

\section{INPUT DATA}

1 - The acceleration model selected for the host vehicle is Linear Decay

2 - Maximum acceleration rate provided by the host vehicle is $3.75 \mathrm{~m} / \mathrm{sec} / \mathrm{sec}$

3 - Equilibrium (crawl) speed of the host vehicle is $40 \mathrm{~m} / \mathrm{sec}$

4 - Length of the host vehicle is $5.25 \mathrm{~m}$

5 - Driver is 28 years old male

6 - The host vehicle is heading straight

7 - Time interval for the sensor is 0.33 seconds

8 - Reflective point detected by the sensor is at Centre of the approaching vehicle

9 - Installation angle for the left sensor is 0 degrees

10- Installation angle for the right sensor is 0 degrees

\section{VEHICLE LOCATIONS AND RESULTS}

\section{Vehicle No. 1}

Location at time $\mathrm{T}$ : distance $165.04 \mathrm{~m}$ and azimuth angle 88.78 degrees

Location at time $\mathrm{T}+\mathrm{t}$ : distance $159.72 \mathrm{~m}$ and azimuth angle 88.74 degrees

Location at time $\mathrm{T}+2 \mathrm{t}$ : distance $154.35 \mathrm{~m}$ and azimuth angle 88.70 degrees

Location at time $\mathrm{T}+3 \mathrm{t}$ : distance $148.91 \mathrm{~m}$ and azimuth angle 88.65 degrees

Approaching from : L

Initial distance $: 165.00$

Offset distance $\quad: 3.45 \mathrm{~m}$

Initial speed $\quad: 16.07 \mathrm{~m} / \mathrm{sec}$

Initial Acceleration : $0.27 \mathrm{~m} / \mathrm{sec} / \mathrm{sec}$

Accel. rate of change: $0.57 \mathrm{~m} / \mathrm{sec} / \mathrm{sec} / \mathrm{sec}$

Acceleration time ( $\mathrm{t} 2)$ : 0.22 seconds

Target time $: 1.37$ seconds

Bullet time $\quad: 6.41$ seconds

Safety margin $\quad:+5.04$ seconds

Non-intersecting path: $\mathrm{N}$

Safe signal : Y

Vehicle No. 2

Location at time $\mathrm{T}$ : distance $175.14 \mathrm{~m}$ and azimuth angle 87.71 degrees

Location at time $\mathrm{T}+\mathrm{t}$ : distance $169.33 \mathrm{~m}$ and azimuth angle 87.63 degrees

Location at time $\mathrm{T}+2 \mathrm{t}$ : distance $163.44 \mathrm{~m}$ and azimuth angle 87.55 degrees

Location at time $\mathrm{T}+3 \mathrm{t}$ : distance $157.48 \mathrm{~m}$ and azimuth angle 87.45 degrees

Approaching from : L

Initial distance $: 175.00$ 
Offset distance $: 7.07 \mathrm{~m}$

Initial speed $\quad: 17.49 \mathrm{~m} / \mathrm{sec}$

Initial Acceleration : $0.80 \mathrm{~m} / \mathrm{sec} / \mathrm{sec}$

Accel. rate of change: $-0.21 \mathrm{~m} / \mathrm{sec} / \mathrm{sec} / \mathrm{sec}$

Acceleration time (t2): 0.30 seconds

Target time $\quad: 1.45$ seconds

Bullet time $\quad: 8.70$ seconds

Safety margin $\quad:+7.25$ seconds

Non-intersecting path: $\mathrm{N}$

Safe signal : Y

Vehicle No. 3

Location at time $\mathrm{T}$ : distance $185.30 \mathrm{~m}$ and azimuth angle 86.75 degrees

Location at time $\mathrm{T}+\mathrm{t}$ : distance $180.05 \mathrm{~m}$ and azimuth angle 86.66 degrees

Location at time $\mathrm{T}+2 \mathrm{t}$ : distance $174.68 \mathrm{~m}$ and azimuth angle 86.55 degrees

Location at time $\mathrm{T}+3 \mathrm{t}$ : distance $169.19 \mathrm{~m}$ and azimuth angle 86.44 degrees

Approaching from : L

Initial distance $: 185.00$

Offset distance $\quad: 10.50 \mathrm{~m}$

Initial speed $\quad: 15.74 \mathrm{~m} / \mathrm{sec}$

Initial Acceleration : $1.16 \mathrm{~m} / \mathrm{sec} / \mathrm{sec}$

Accel. rate of change: $-0.11 \mathrm{~m} / \mathrm{sec} / \mathrm{sec} / \mathrm{sec}$

Acceleration time (t2): 0.37 seconds

Target time $\quad: 1.52$ seconds

Bullet time $\quad: 8.42$ seconds

Safety margin $\quad:+6.90$ seconds

Non-intersecting path: $\mathrm{N}$

Safe signal : Y

Vehicle No. 4

Location at time $\mathrm{T}$ : distance $165.85 \mathrm{~m}$ and azimuth angle 84.20 degrees

Location at time $\mathrm{T}+\mathrm{t}$ : distance $160.75 \mathrm{~m}$ and azimuth angle 84.02 degrees

Location at time $\mathrm{T}+2 \mathrm{t}$ : distance $155.62 \mathrm{~m}$ and azimuth angle 83.82 degrees

Location at time $\mathrm{T}+3 \mathrm{t}$ : distance $150.48 \mathrm{~m}$ and azimuth angle 83.61 degrees

Approaching from $: \mathrm{R}$

Initial distance $: 165.02$

Offset distance $\quad: 16.62 \mathrm{~m}$

Initial speed $\quad: 15.46 \mathrm{~m} / \mathrm{sec}$

Initial Acceleration : $0.52 \mathrm{~m} / \mathrm{sec} / \mathrm{sec}$

Accel. rate of change: $-0.64 \mathrm{~m} / \mathrm{sec} / \mathrm{sec} / \mathrm{sec}$

Acceleration time (t2): 0.50 seconds

Target time $: 1.66$ seconds

Bullet time $\quad:-15.64$ seconds

Safety margin $\quad:-17.30$ seconds

Non-intersecting path: $\mathrm{N}$

Safe signal : $\mathrm{Y}$

Vehicle No. 5

Location at time $\mathrm{T}$ : distance $215.95 \mathrm{~m}$ and azimuth angle 84.62 degrees

Location at time $\mathrm{T}+\mathrm{t}$ : distance $210.91 \mathrm{~m}$ and azimuth angle 84.49 degrees 
Location at time $\mathrm{T}+2 \mathrm{t}$ : distance $205.82 \mathrm{~m}$ and azimuth angle 84.35 degrees Location at time $\mathrm{T}+3 \mathrm{t}$ : distance $200.67 \mathrm{~m}$ and azimuth angle 84.21 degrees

Approaching from $: \mathrm{R}$

Initial distance $: 215.00$

Offset distance $: 20.22 \mathrm{~m}$

Initial speed $\quad: 15.27 \mathrm{~m} / \mathrm{sec}$

Initial Acceleration : $0.42 \mathrm{~m} / \mathrm{sec} / \mathrm{sec}$

Accel. rate of change: $0.18 \mathrm{~m} / \mathrm{sec} / \mathrm{sec} / \mathrm{sec}$

Acceleration time (t2): 0.58 seconds

Target time $\quad: 1.73$ seconds

Bullet time $\quad: 9.43$ seconds

Safety margin $\quad:+7.69$ seconds

Non-intersecting path: $\mathrm{N}$

Safe signal : Y

Vehicle No. 6

Location at time $\mathrm{T}$ : distance $179.58 \mathrm{~m}$ and azimuth angle 82.40 degrees Location at time $\mathrm{T}+\mathrm{t}$ : distance $174.58 \mathrm{~m}$ and azimuth angle 82.18 degrees Location at time $\mathrm{T}+2 \mathrm{t}$ : distance $169.58 \mathrm{~m}$ and azimuth angle 81.95 degrees Location at time $\mathrm{T}+3 \mathrm{t}$ : distance $164.56 \mathrm{~m}$ and azimuth angle 81.70 degrees Approaching from : R

Initial distance $: 178.00$

Offset distance $\quad: 23.80 \mathrm{~m}$

Initial speed $\quad: 15.31 \mathrm{~m} / \mathrm{sec}$

Initial Acceleration : $-0.20 \mathrm{~m} / \mathrm{sec} / \mathrm{sec}$

Accel. rate of change: $0.66 \mathrm{~m} / \mathrm{sec} / \mathrm{sec} / \mathrm{sec}$

Acceleration time ( $\mathrm{t} 2)$ : 0.66 seconds

Target time $\quad: 1.81$ seconds

Bullet time $\quad: 7.17$ seconds

Safety margin $\quad:+5.36$ seconds

Non-intersecting path: $\mathrm{N}$

Safe signal $\quad: Y$

Results

1- Perception-reaction time of the turning driver is 1.15 seconds

2 - The nearest vehicle is on distance $148.87 \mathrm{~m}$ and speed $16.62 \mathrm{~m} / \mathrm{s}$

3- Correction factor for the maximum acceleration rate is 0.57

4- Departure acceleration rate of the turning vehicle is $2.12 \mathrm{~m} / \mathrm{s} / \mathrm{s}$

\section{FINAL DECESION:}

Should the warning signal continue: $\mathrm{N}$ 


\section{Appendix "G"}

MATLAB Code for Constant Acceleration 
$\%$ MATLAB code to validate TWSC intersections by simulating detection sensors $\%$ by: Essam Dabbour

clc

clear all

\%Input vehicle data

POL=input('Input the model of acceleration used for host vehicle (Constant / Linear decay) [C]: ','s'); if isempty(POL)

$\mathrm{POL}=\mathrm{C}^{\mathrm{C}}$;

end

if $\mathrm{POL}==^{\prime} \mathrm{C}^{\prime}$

$\mathrm{AV}=$ input('Input the maximum acceleration provided by the host vehicle in $\mathrm{m} / \mathrm{sec} / \mathrm{sec}$ [2.75]: ');

if isempty(AV)

$\mathrm{AV}=2.75$

end

end

if $\mathrm{POL}==^{\prime} \mathrm{L} '$

$\mathrm{AV}=$ input('Input the maximum initial acceleration provided by the host vehicle in $\mathrm{m} / \mathrm{sec} / \mathrm{sec}$ [2.75]: ');

if isempty $(\mathrm{AV})$

$\mathrm{AV}=2.75$

end

$\mathrm{EQ}=$ input('Input the equilibrium (crawl) speed of the host vehicle in $\mathrm{m} / \mathrm{sec}$ [24]: ');

if isempty(EQ)

$\mathrm{EQ}=24$;

end

end

$\mathrm{L}=$ input('Input the length of the host vehicle in meters [5.25 m]: ');

if isempty(L)

$\mathrm{L}=5.25$;

end

\%Input driver data

AGE=input('Input the age of the driver in years [28]: ');

if isempty(AGE)

$$
\mathrm{AGE}=28 \text {; }
$$

end

GENDER=input('Input the gender of the driver M/F [M]: ','s');

if isempty(GENDER)

GENDER = 'M';

end

if GENDER == 'M'

GGENDER=0;

end

if GENDER $==$ ' $\mathrm{F}$ '

GGENDER=1;

end

TURN=input('Input driver turning signal L/R or "N" for no signal [N]: ','s');

if isempty(TURN) 


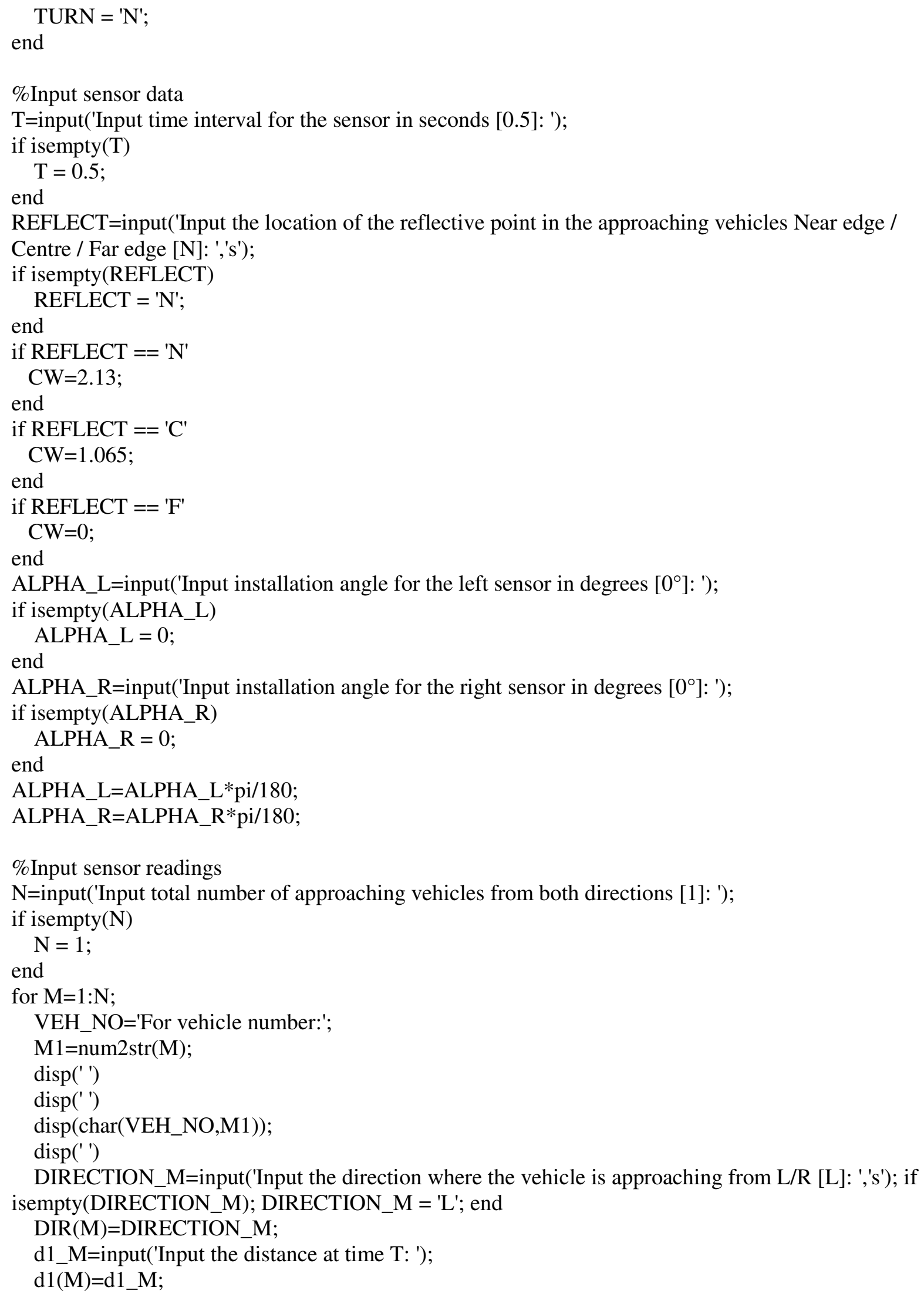


THETA1_M=input('Input azimuth angle at time T: ');

THETA1_M=THETA1_M*pi/180;

THETA1(M)=THETA1_M;

d2_M=input('Input the distance at time $\mathrm{T}+\mathrm{t}$ : ');

$\mathrm{d} 2(\mathrm{M})=\mathrm{d} 2 \_\mathrm{M}$;

THETA2_M=input('Input azimuth angle at time $\mathrm{T}+\mathrm{t}$ : ');

THETA2_M=THETA2_M*pi/180;

THETA2 $(\mathrm{M})=$ THETA2_M;

d3_M=input('Input the distance at time $\mathrm{T}+2 \mathrm{t}$ : ');

$\mathrm{d} 3(\mathrm{M})=\mathrm{d} 3 \mathrm{M}$;

THETA3_M=input('Input azimuth angle at time $\mathrm{T}+2 \mathrm{t}$ : ');

THETA3_M=THETA3_M*pi/180;

THETA3(M)=THETA3_M;

$\%$ Safe signal if the equipped vehicle is turning right and the

$\%$ approaching vehicle is approaching from right

$\operatorname{SIG}(\mathrm{M})=$ 'N'; INT(M)='N';

if TURN=='R'\&\& DIRECTION_M=='R'

Tbullet(M)=0; SIG(M)='Y'; INT(M)='Y';

end

$\%$ Calculate speed and acceleration

$\mathrm{dv} 1(\mathrm{M})=\operatorname{sqrt}((\mathrm{d} 1(\mathrm{M}) * \mathrm{~d} 1(\mathrm{M}))+(\mathrm{d} 2(\mathrm{M}) * \mathrm{~d} 2(\mathrm{M}))-2 * \mathrm{~d} 1(\mathrm{M}) * \mathrm{~d} 2(\mathrm{M}) * \cos ($ THETA1(M)-THETA2(M)));

$\mathrm{dv} 2(\mathrm{M})=\operatorname{sqrt}((\mathrm{d} 2(\mathrm{M}) * \mathrm{~d} 2(\mathrm{M}))+(\mathrm{d} 3(\mathrm{M}) * \mathrm{~d} 3(\mathrm{M}))-2 * \mathrm{~d} 2(\mathrm{M}) * \mathrm{~d} 3(\mathrm{M}) * \cos ($ THETA2(M)-THETA3(M)));

$\mathrm{a}(\mathrm{M})=(\mathrm{dv} 2(\mathrm{M})-\mathrm{dv} 1(\mathrm{M})) /\left(\mathrm{T}^{*} \mathrm{~T}\right)$;

$\mathrm{v} 0(\mathrm{M})=(1.5 * \mathrm{dv} 1(\mathrm{M})-0.5 * \mathrm{dv} 2(\mathrm{M})) / \mathrm{T}$;

$\mathrm{v} 2(\mathrm{M})=\mathrm{v} 0(\mathrm{M})+2 * \mathrm{~T} * \mathrm{a}(\mathrm{M})$;

$\mathrm{wf} 1(\mathrm{M})=\mathrm{d} 1(\mathrm{M}) * \mathrm{~d} 2(\mathrm{M}) * \sin ($ THETA1(M)-THETA2(M))/dv1(M);

$\mathrm{wf} 2(\mathrm{M})=\mathrm{d} 2(\mathrm{M}) * \mathrm{~d} 3(\mathrm{M}) * \sin ($ THETA2(M)-THETA3(M))/dv2(M);

$\mathrm{wf}(\mathrm{M})=(\mathrm{wf} 1(\mathrm{M})+\mathrm{wf} 2(\mathrm{M})) / 2$;

$\operatorname{df}(M)=\operatorname{sqrt}(d 3(M) * d 3(M)-w f(M) * w f(M))$;

$\%$ Calculate Tbullet

Tbullet $(\mathrm{M})=0$;

if $\operatorname{SIG}(\mathrm{M})=={ }^{\prime} \mathrm{N}^{\prime}$

$\mathrm{p}=\left[0.5^{*} \mathrm{a}(\mathrm{M}) \mathrm{v} 2(\mathrm{M})-\mathrm{df}(\mathrm{M})\right]$;

$\mathrm{y}=\operatorname{roots}(\mathrm{p})$;

for hh=1:numel(y)

if $\operatorname{imag}(\mathrm{y}(\mathrm{hh}))==0$

$\operatorname{Tbullet}(\mathrm{M})=\mathrm{y}(\mathrm{hh})$;

end

end

end

\%Initial distance of the vehicle from intersection

$\mathrm{d} 0(\mathrm{M})=\mathrm{dv} 1(\mathrm{M})+\mathrm{dv} 2(\mathrm{M})+\mathrm{df}(\mathrm{M})$;

end

$\%$ Eliminate zero values 


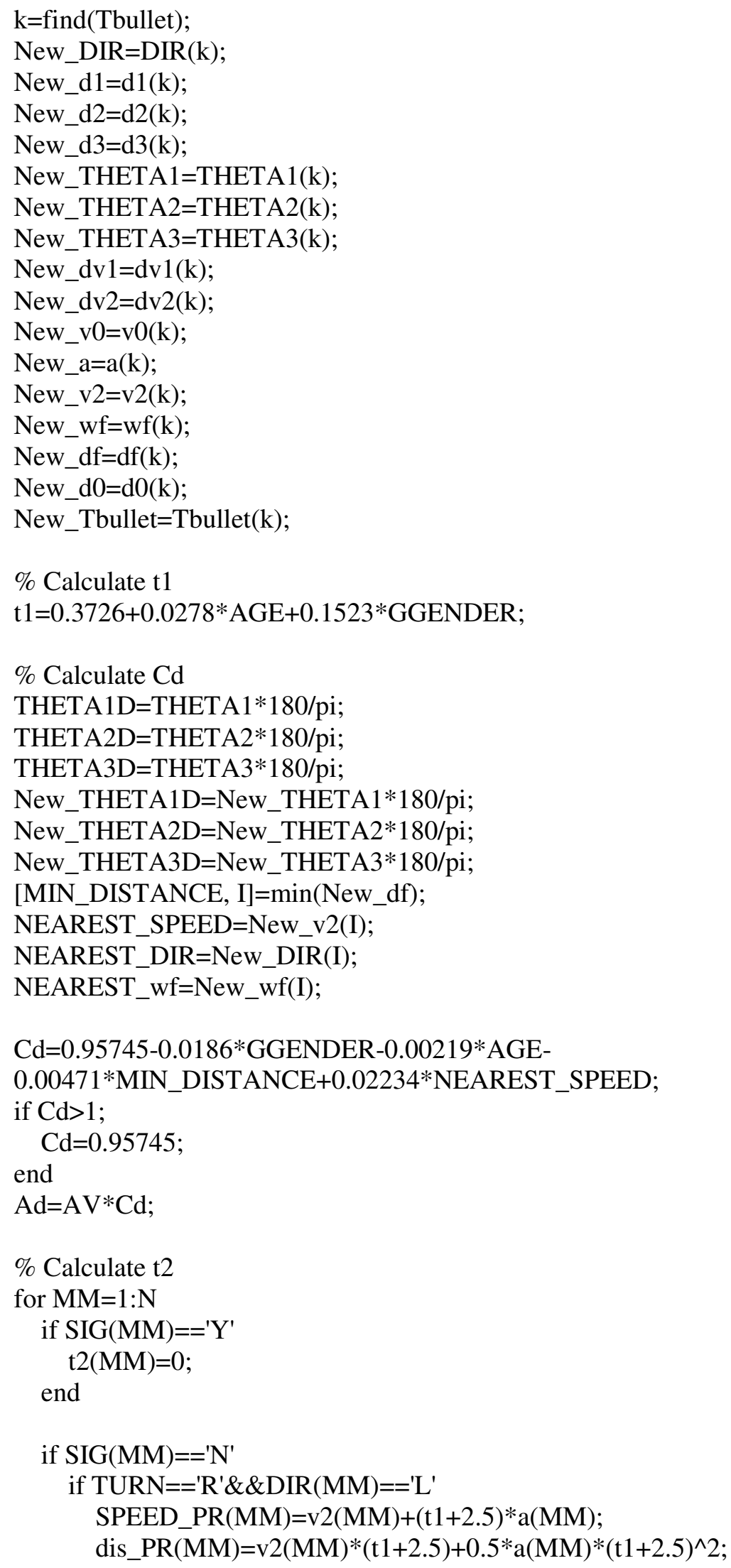




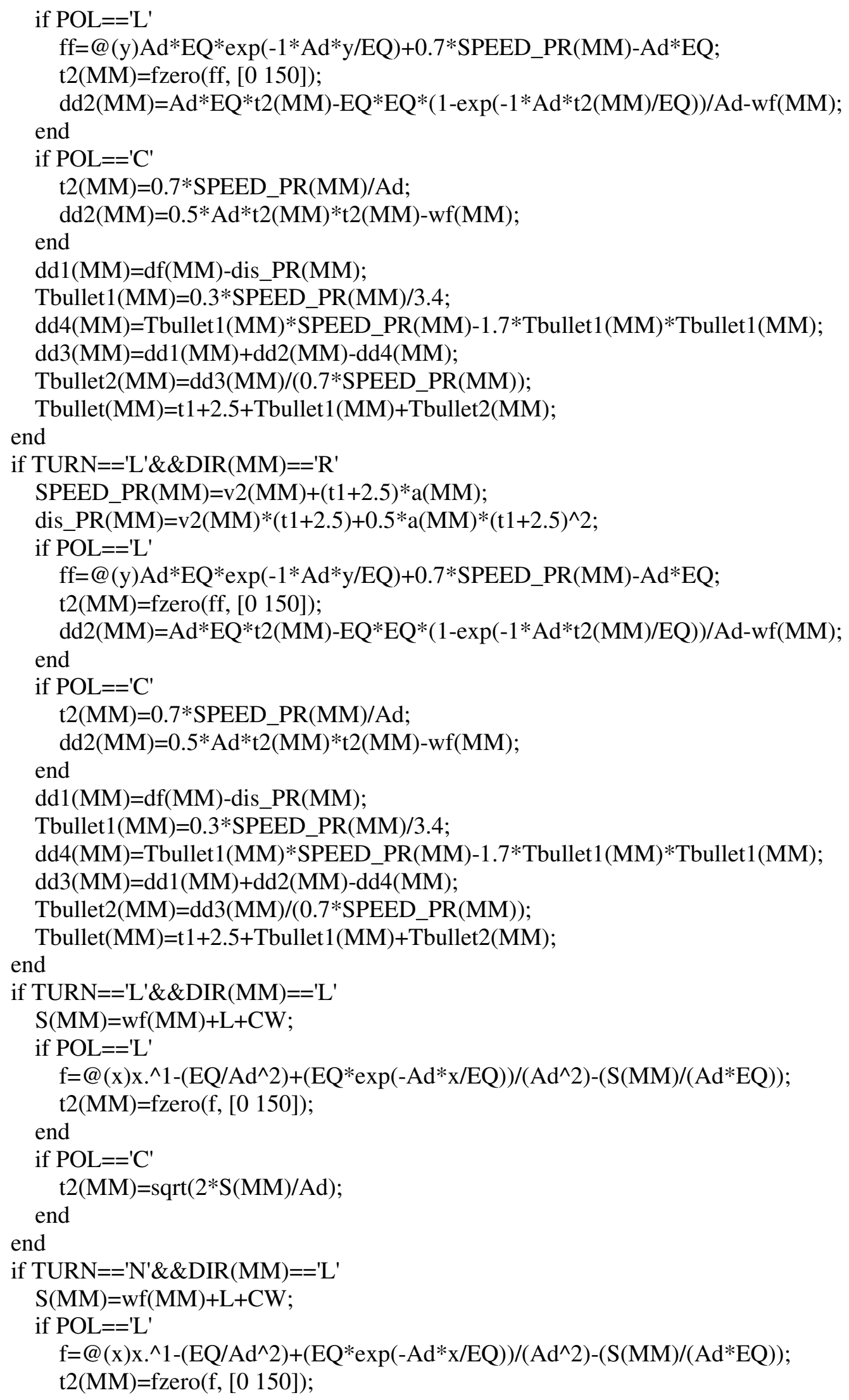




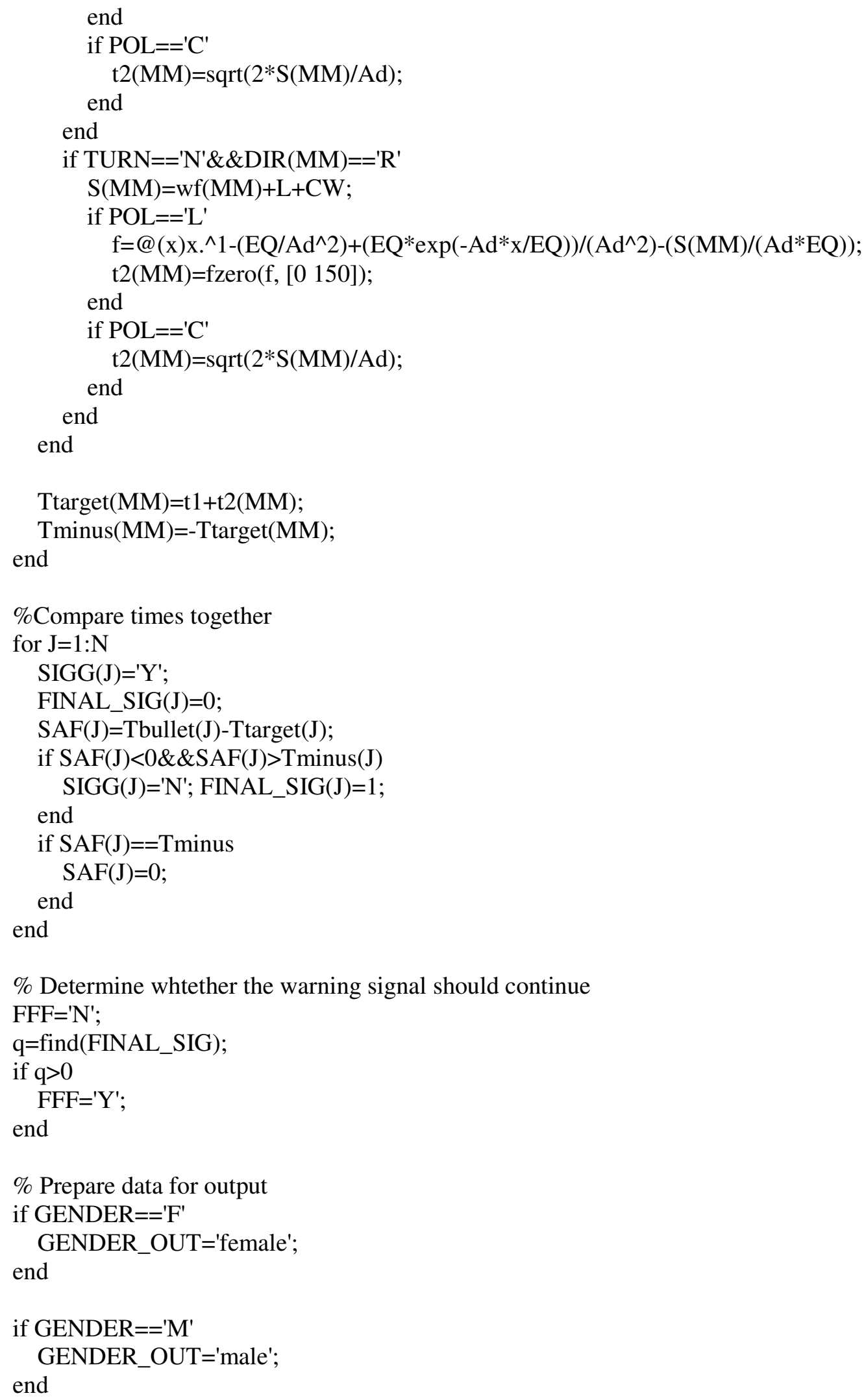




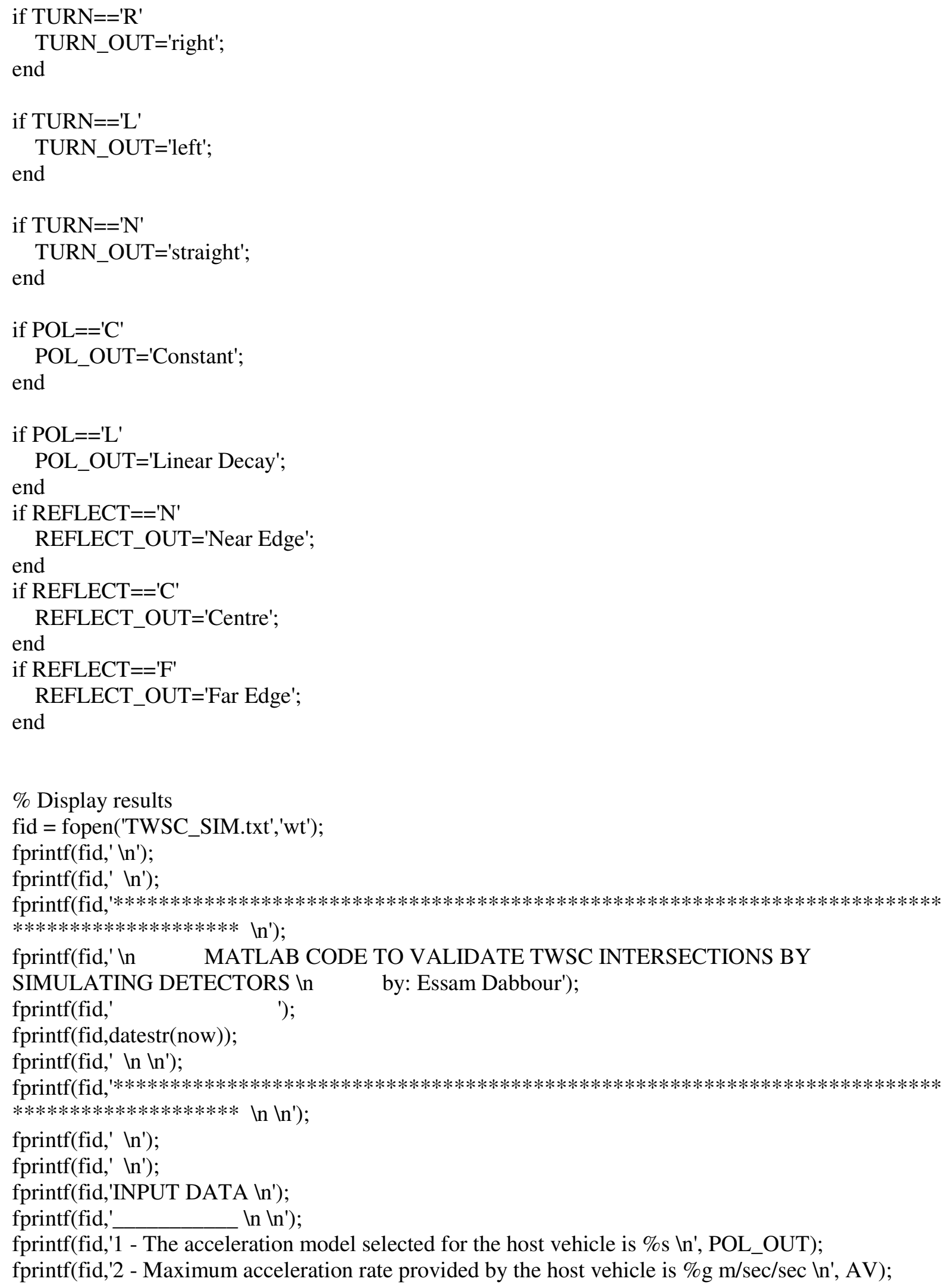




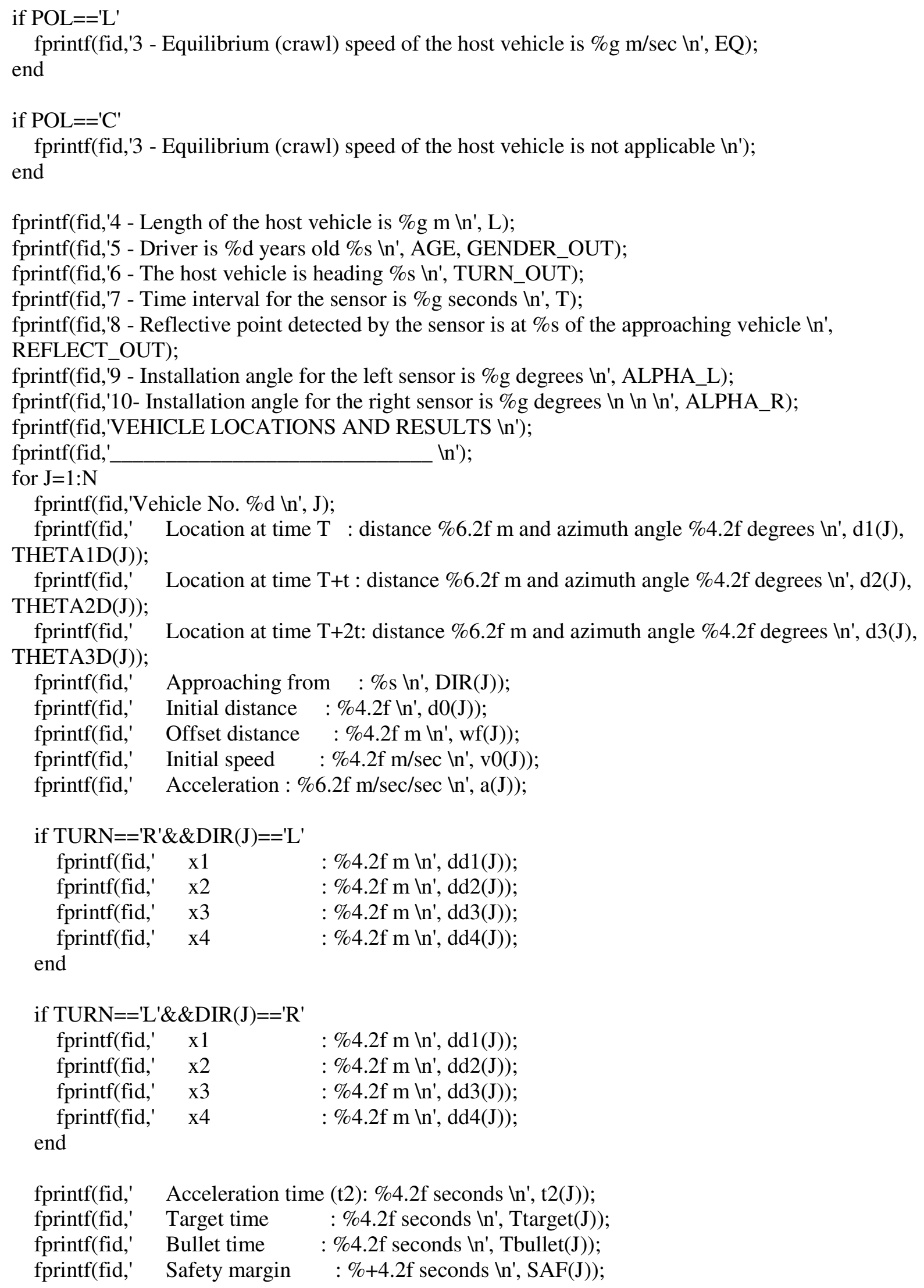


fprintf(fid,' Non-intersecting path: \%s $\ln$ ', INT(J));

end

fprintf(fid,' $\quad$ Safe signal $\%$ s $\backslash n \backslash n$ ', $\operatorname{SIGG}(J))$;

fprintf(fid,'In $\backslash n ')$;

fprintf(fid,'Results $\backslash n$ ');

fprintf(fid,' $\ln \backslash n ')$;

fprintf(fid,' 1- Perception-reaction time of the turning driver is \% $4.2 \mathrm{f}$ seconds $\backslash \mathrm{n}$ ', $\mathrm{t} 1$ );

fprintf(fid,' 2- The nearest vehicle is on distance $\% 6.2 \mathrm{f} \mathrm{m}$ and speed $\% 4.2 \mathrm{f} \mathrm{m} / \mathrm{s} \backslash \mathrm{n}$,

MIN_DISTANCE, NEAREST_SPEED);

fprintf(fid,' 3- Correction factor for the maximum acceleration rate is $\% 4$.2f $\backslash \mathrm{n}$ ', Cd);

fprintf(fid,' 4- Departure acceleration rate of the turning vehicle is $\% 4.2 \mathrm{f} \mathrm{m} / \mathrm{s} / \mathrm{s} \backslash \mathrm{n} \backslash \mathrm{n} \backslash \mathrm{n}$ ', Ad);

fprintf(fid,'FINAL DECESION: $\backslash n$ ');

fprintf(fid,'_ $\ln \backslash n \backslash n '$ );

fprintf(fid,'Should the warning signal continue: \%s \n', FFF);

fprintf(fid,' 'In $\backslash n$ ');

fprintf(fid, ${ }^{\prime} * * * * * * * * * * * * * * * * * * * * * * * * * * * * * * * * * * * * * * * * * * * * * * * * * * * * * * * * * * * * * * * * * * * * * * * * * * *$

$\left.* * * * * * * * * * * * * * * * * * * * \quad \mathrm{n}^{\prime}\right)$;

fclose(fid); 